\title{
Rates of Vertical Mixing, Gas Exchange, and New Production: Estimates from Seasonal Gas Cycles in the Upper Ocean Near Bermuda
}

by

\author{
William Seth Spitzer \\ B.A., cum laude, Chemistry and Physics \\ Harvard University \\ (1983) \\ Submitted in partial fulfillment of the \\ requirements for the degree of \\ Doctor of Philosophy \\ at the
}

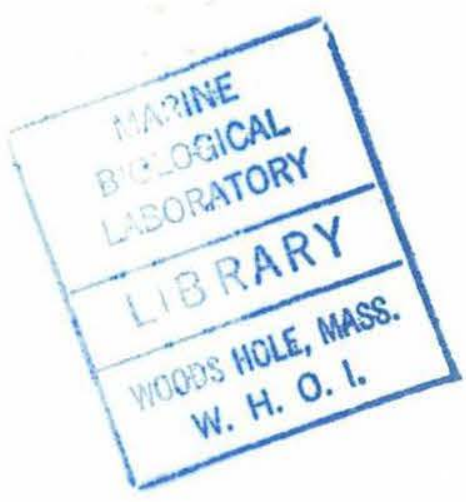
MASSACHUSETTS INSTITUTE OF TECHNOLOGY
and the

WOODS HOLE OCEANOGRAPHIC INSTITUTION

June 1989

(C) William S. Spitzer, 1989

The author hereby grants to MIT and to WHOI permission to reproduce and to distribute copies of this thesis document in whole or in part.

Signature of Author

Joint Program in Chemical Oceanography Massachusetts Institute of Technology Woods Hole Oceanographic Institution

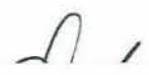
May 30, 1989

Certified by Accepted by

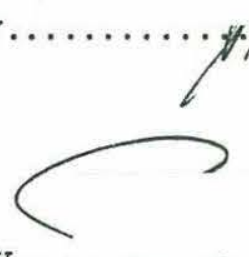

William J. Jenkins

Senior Scienłist, Woods Hole Oceanographic Institution Thesis Supervisor

Philip M. Gschwend

Chairman, Joint Committee for Chemical Oceanography

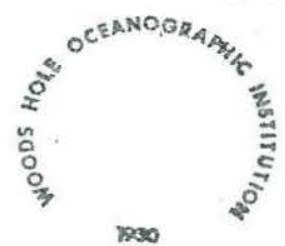

Massachusetts Institute of Technology Woods Hole Oceanographic Institution 


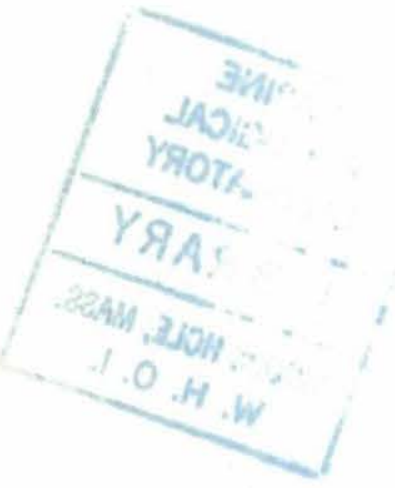




\title{
Rates of Vertical Mixing, Gas Exchange, and New Production: Estimates from Seasonal Gas Cycles in the Upper Ocean Near Bermuda
}

by

\author{
William Seth Spitzer
}

\author{
Submitted to the Massachusetts Institute of Technology- \\ Woods Hole Oceanographic Institution \\ Joint Program in Chemical Oceanography \\ on May 29, 1989, in partial fulfillment of the \\ requirements for the degree of \\ Doctor of Philosophy
}

\begin{abstract}
Argon measurements, obtained from three years of monthly detailed vertical profiles near Bermuda (Station $\mathrm{S}, 32^{\circ} \mathrm{N} 64^{\circ} \mathrm{W}$ ), show a maximum in argon supersaturation of about $4 \%$ in the seasonal thermocline in late summer. Since the argon supersaturation is 3-4 times smaller than that of oxygen, most of the oxygen supersaturation is not of physical origin and hence must result from biological production.

In the winter mixed layer, air injection produces argon supersaturation despite high gas exchange rates. During spring and summer, radiative heating, air injection, and an upward argon flux create an even larger supersaturation in the mixed layer. In the seasonal thermocline, radiative heating creates argon supersaturations that persist in spite of vertical mixing.

The observed seasonal cycles of temperature, argon, helium, and oxygen are simulated with an upper ocean model. I linearize the model's response to variations in vertical diffusivity, air injection, gas exchange rate, and new production and then use an inverse technique (singular value decomposition) to determine the values of these parameters that best fit the data. Results for the 1985-1987 average are as follows: A vertical turbulent diffusivity of $1.0 \pm 0.1 \times 10^{-4} \mathrm{~m}^{2}$ $\mathrm{s}^{-1}$ is consistent with both the thermal history and subsurface argon distribution. The rate of air injection, determined to $\pm 15 \%$, is similar to previous estimates. The seasonally-averaged gas exchange rate, determined to $\pm 11 \%$, is consistent within errors with that predicted by Liss and Merlivat (1986). I estimate a lower limit to depth-integrated new production below the mixed layer of $5.0 \pm 1.0$ moles $\mathrm{O}_{2} \mathrm{~m}^{-2} \mathrm{yr}^{-1}$, and obtain an estimate of $6.2 \pm 0.9 \mathrm{moles}_{2} \mathrm{~m}^{-2}$ $\mathrm{yr}^{-1}$ if new production in the mixed layer is fixed at zero. The period 1985-1987 appears to be typical of the climatological mean conditions at Station S and comparable to the 1960-1970 average period analyzed by Jenkins \& Goldman (1985) and Musgrave et al. (1988).

I propose that a mesoscale anticyclonic eddy is responsible for excess ${ }^{3} \mathrm{He}$ and nitrate in the euphotic zone observed at a July, 1986 occupation of the Station S site. Hydrographic profiles are consistent with a type of eddy observed by Brundage \& Dugan (1986), characterized by an unusually thick lens of subtropical mode $\left(18^{\circ} \mathrm{C}\right)$ water. Analysis of the 35 year hydrographic record suggests that such eddies may arrive at Station $\mathrm{S}$ with an average frequency of 2-6 times per year, mostly during the summer and in years of vigorous $18^{\circ} \mathrm{C}$ water formation. Their timing and character suggest that they may be formed during winter convection events in the
\end{abstract}


northeastern Sargasso Sea, advected southwestward by the gyre-scale circulation, and eventually absorbed by the Gulf Stream. Their magnitude and frequency indicate that they may supply a significant portion of the ${ }^{3} \mathrm{He}$ and nutrient flux into the euphotic zone near Bermuda, and suggest a mechanism by which newly formed subtropical mode water is incorporated within the gyre interior. However, enhanced new production in such eddies could account for only a small portion of the new production integrated over the Sargasso Sea.

Thesis Supervisor: William J. Jenkins

Title: Senior Scientist, Woods Hole Oceanographic Institution 


\section{Acknowledgements}

I am especially thankful to have had the opportunity to work closely with Bill Jenkins, who has been a source of inspiration, encouragement, and guidance. I would like to thank the staff of the Helium Isotope Lab, especially Dempsey Lott and Marcia Davis, for their invaluable support. The members of my thesis committee - Ed Boyle, Dave Musgrave, and Don Anderson - provided valuable constructive criticism. And, I have benefitted from the expertise and patience of many others, including Nelson Hogg, Bob Weller, Scott Doney, Dave Glover, Ellen Druffel, Mike Bender, and Terry Joyce. This work would have been impossible without the dedicated efforts of Tim Jickells and Rachael Sheriff-Dow at the Bermuda Biological Station and the seamanship of Captain Michael Rhodes and Engineer Tony Sheriff of the R/V Weatherbird. Special thanks go to George Halliwell at Univ. of Rhode Island for providing the FNOC wind data. This project has been supported by grant OCE85-01171 from the National Science Foundation. 


\section{Contents}

1 Introduction $\quad 13$

1.1 New Production . . . . . . . . . . . . . . . . . . 13

1.1 .1 Classical Estimates . . . . . . . . . . . . . . . . 13

1.1.2 Tracer-based Estimates . . . . . . . . . . . . . . . 14

1.2 Constraining Physical Processes with Noble Gases . . . . . . . . . . . . . 21

1.2.1 Argon as an Abiogenic Analog for Oxygen . . . . . . . . . . . . . . 22

1.2.2 Sources of Saturation Anomalies . . . . . . . . . . . . 23

1.2.3 Gas Exchange. . . . . . . . . . . . . . . . . . . 24

1.3 Supplying the Upward Flux of Nutrients . . . . . . . . . . . . . . . 27

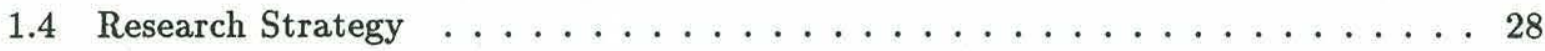

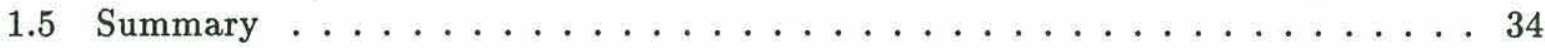

2 Methods $\quad 36$

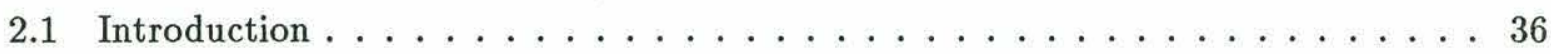

2.2 Argon Measurements in Seawater . . . . . . . . . . . . . 36

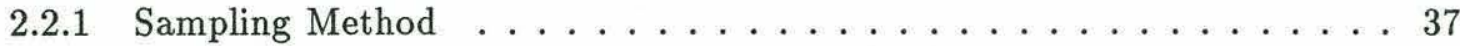

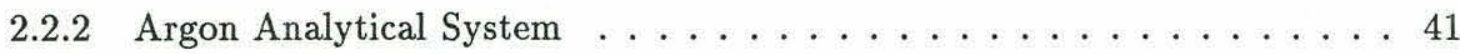

2.2 .3 Extraction ............................... 41

2.2.4 Purification \& Diagnostic $\mathrm{He} / \mathrm{Ne}$ Analysis . . . . . . . . . . . . . . . 44

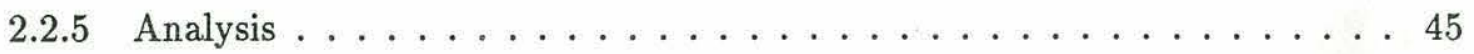

2.2 .6 Semi-automated Operation . . . . . . . . . . . . 47

2.2.7 Standard Preparation . . . . . . . . . . . . . . . . 48 
2.2.8 Peak Height Manometry . . . . . . . . . . . . . . . 54

2.2 .9 Improvements Using Isotope Dilution $\ldots \ldots \ldots \ldots$

2.2 .10 Data Reduction . . . . . . . . . . . . . . . . . 58

2.2.11 Peak Height Manometry Results . . . . . . . . . . . . . . 61

2.2 .12 Isotope Dilution Results . . . . . . . . . . . . . . . 62

2.2 .13 Sampling Errors . . . . . . . . . . . . . . . . 62 62

2.3 Tritium, Helium and Neon Analyses . . . . . . . . . . . . 68

2.4 Hydrographic Data . . . . . . . . . . . . . . . . . . 68

3 Rates of Vertical Mixing, Gas Exchange, and New Production 73

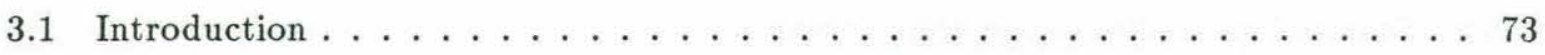

3.2 Methods . . . . . . . . . . . . . . . . . . . 74

3.3 Results . . . . . . . . . . . . . . . . . . . . 75

3.4 A Seasonal Upper Ocean Model . . . . . . . . . . . . . . . . . . . . . 80

3.5 Linearization of Model Response . . . . . . . . . . . . . . . . 83

3.5 .1 Vertical mixing . . . . . . . . . . . . . 85

3.5.2 Gas exchange and air injection . . . . . . . . . . 89

3.5.3 Biological oxygen production . . . . . . . . . . . 92

3.6 Quantitative Estimates . . . . . . . . . . . . . . . . 93

3.7 Discussion . . . . . . . . . . . . . . . . . . 97

3.7 .1 Vertical mixing . . . . . . . . . . . . . 97

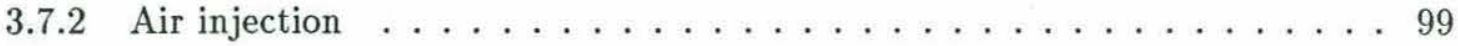

3.7 .3 Gas exchange ........................ 100

3.7 .4 Biological oxygen production . . . . . . . . . . . 102

3.7.5 Lateral transport and Interannual Variations . . . . . . . . . . 106

3.7 .6 Future Work . . . . . . . . . . . . . . . . 110

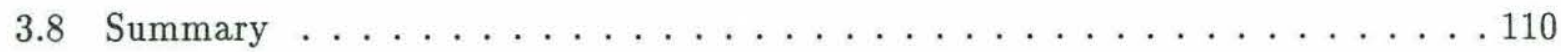

4 Nutrient Injection into the Euphotic Zone $\quad 113$

4.1 Introduction . . . . . . . . . . . . . . . . . . . . . . . . . .

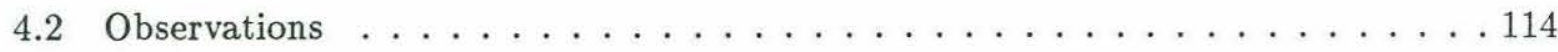


4.3 Approach . . . . . . . . . . . . . . . . . . . 124

4.4 Results. . . . . . . . . . . . . . . . . . . . . . . . . . . . . . . . . . .

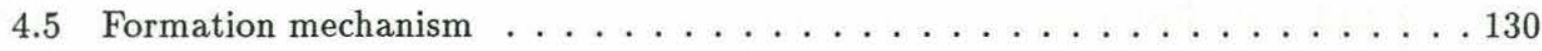

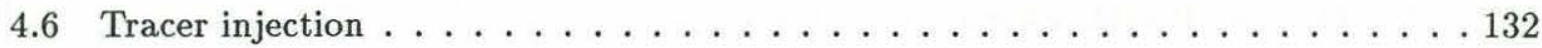

4.7 Comparison with other tracer sources $\ldots \ldots \ldots \ldots \ldots \ldots \ldots$

4.8 Implications for mode water renewal \& new production $\ldots \ldots \ldots$. . . . . . . . 134

4.9 Summary \& speculations . . . . . . . . . . . . . . . . 136

$\begin{array}{lll}5 & \text { Summary } & 138\end{array}$

5.1 Analytical developments . . . . . . . . . . . . . . . 138

5.2 Seasonal gas cycles in the upper ocean . . . . . . . . . . . . 138

5.3 Quantitative estimates of vertical mixing, gas exchange, and new production . . 139

5.4 Upward flux of nutrients via eddies . . . . . . . . . . . . . . . . 140

6 Bibliography $\quad 142$

$\begin{array}{ll}\text { A Measured Argon Concentrations } & 151\end{array}$ 


\section{List of Figures}

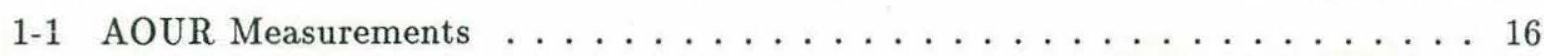

$1-2$ Seasonal cycles at Bermuda . . . . . . . . . . . . . . . . 19

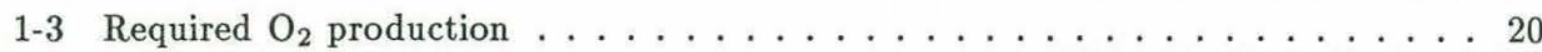

$1-4$ Physical properties of gases . . . . . . . . . . . . . 21

$1-5$ Gas transfer rates . . . . . . . . . . . . . . . . 26

$1-6$ Location of sampling site . . . . . . . . . . . . . . . . 29

1-7 Annual displacements at $159 \mathrm{~m} \ldots \ldots \ldots \ldots \ldots$

$1-8$ Net annual heat flux . . . . . . . . . . . . . . . . 31

1-9 Time constants for mixed layer gas exchange $\ldots \ldots \ldots \ldots$

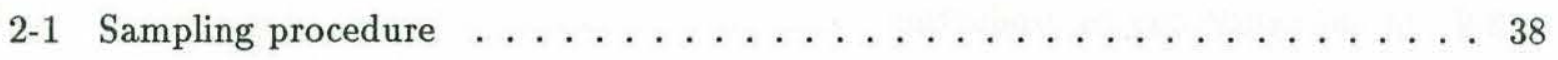

2-2 Argon sampling experiments . . . . . . . . . . . . 40

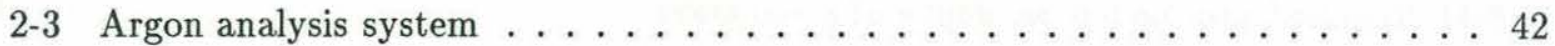

2-4 Diagnostic He and Ne measurements . . . . . . . . . . . . . . . 46

2-5 Standard preparation line . . . . . . . . . . . . . . 52

$2-6$ Manometer non-linearity . . . . . . . . . . . . . . . . 53

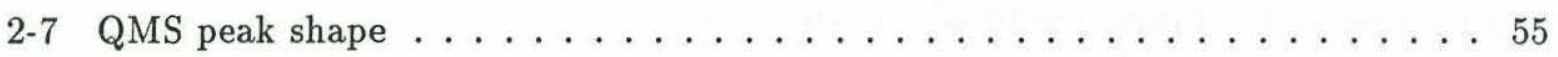

$2-8$ Repeat totes experiment . . . . . . . . . . . . . . . 5 57

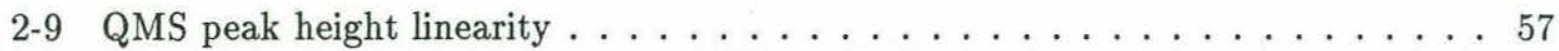

2-10 QMS peak height ratio linearity . . . . . . . . . . . . . . . 60

2-11 Isotope discrimination vs. standard number . . . . . . . . . 63

2-12 Air standard depletion . . . . . . . . . . . . . . . . . 63

2-13 Deep argon measurements . . . . . . . . . . . . . . . 64 64 
2-14 Measured argon supersaturations . . . . . . . . . . . . . 65

$2-15$ Replicate precision . . . . . . . . . . . . . . . 65

2-16 Detecting air contamination . . . . . . . . . . . . 66

2-17 Salinity and oxygen data vs. density . . . . . . . . . . . . . . 70

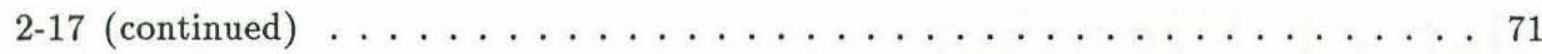

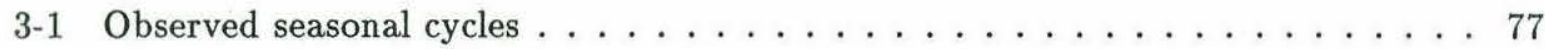

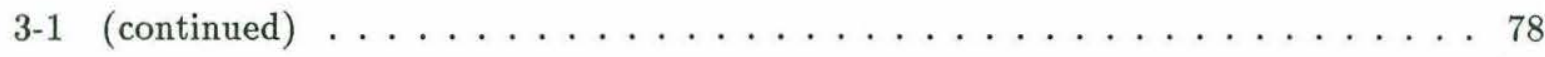

$3-2$ Mixed layer cycles $\ldots \ldots \ldots \ldots \ldots \ldots \ldots \ldots$

$3-3$ FNOC wind speeds . . . . . . . . . . . . . . . . . 81

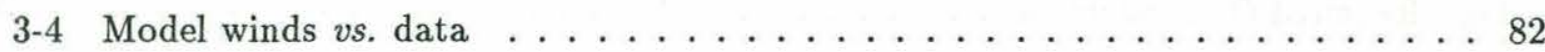

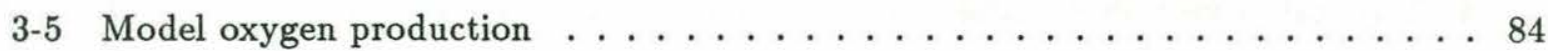

$3-6$ Simulated seasonal cycles $\ldots \ldots \ldots \ldots \ldots$

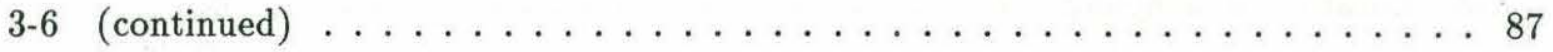

3-7 Model sensitivity to vertical diffusivity . . . . . . . . . . 88

3-8 Model sensitivity to gas exchange, air injection . . . . . . . . . . . 90

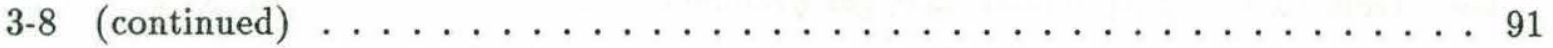

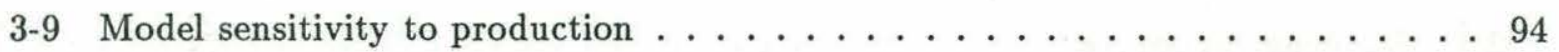

3-10 Model gas exchange and air injection fluxes . . . . . . . . . . . . 101

3-11 Required production vs, depth of production . . . . . . . . . . . 104

3-12 Required production vs. time of production . . . . . . . . . . . . 105

3-13 Mixed layer depths at Station S . . . . . . . . . . . . . . . 108

3-14 Heat content and heat flux at Station $\mathrm{S} \ldots \ldots \ldots$. . . . . . . . . . 109

3-15 Sensitivity of inversion to additional gases . . . . . . . . . . . . . 111

$4-1$ Station 593 profiles . . . . . . . . . . . . . . . . . 115

4-2 Station 593 property vs. temperature plots . . . . . . . . . . 116

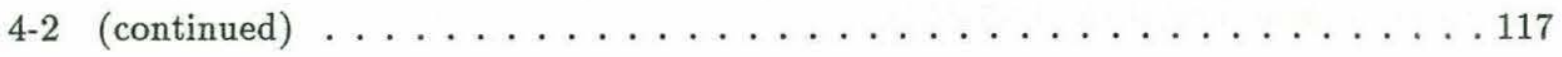

$4-3$ Vertical displacement field . . . . . . . . . . . . . . . 118

$4-4$ Mode water properties . . . . . . . . . . . . . . . . . . . 119

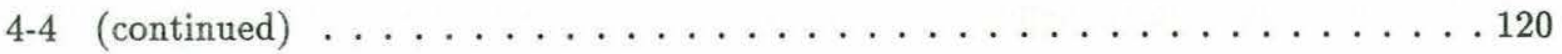




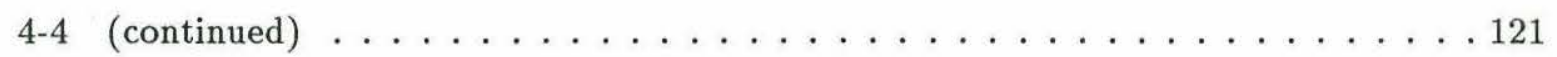

$4-5$ Mode water property-property plots . . . . . . . . . . . . . 122

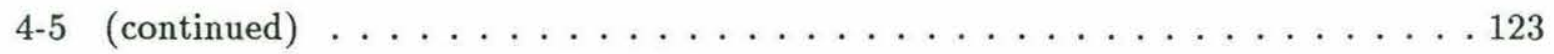

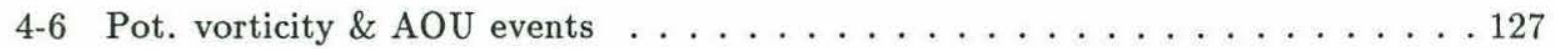

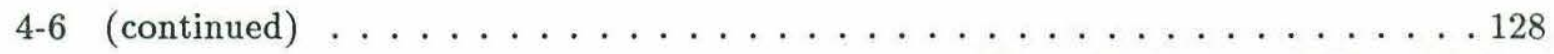

$4-7$ Power spectra . . . . . . . . . . . . . . . . . . . . . . 129

$4-8$ Sampling frequency . . . . . . . . . . . . . . . . . 130

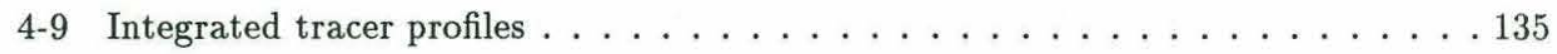




\section{List of Tables}

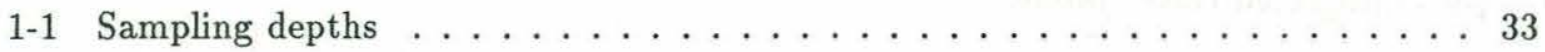

2-1 Argon sampling experiments . . . . . . . . . . . . . . . 40

2-2 Extraction efficiency experiments . . . . . . . . . . . . . . 44

2-3 Procedure for standards \& blanks . . . . . . . . . . . . . . . . . 49

$2-4$ Procedure for seawater samples . . . . . . . . . . . . . . 50

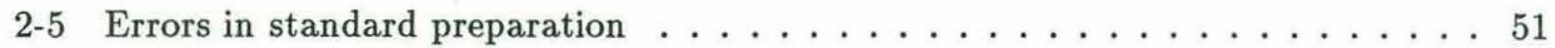

2-6 Random and systematic measurement errors . . . . . . . . . . . . 67

2-7 Precision for tritium and helium analyses $\ldots \ldots \ldots 6$

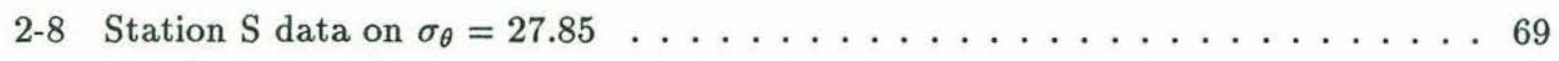

$2-9$ Salinity and oxygen data $v s$, density $\ldots \ldots \ldots \ldots \ldots \ldots$

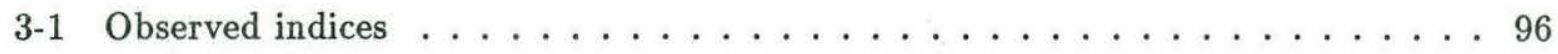

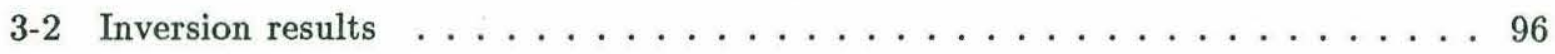

3-3 Errors associated with the constraint equations $\ldots \ldots \ldots . \ldots 9$

$3-4$ Weighted coefficient matrix $\ldots \ldots \ldots \ldots \ldots \ldots \ldots$

$4-1$ Subtropical mode water properties . . . . . . . . . . . . . . . . 124 


\section{Chapter 1}

\section{Introduction}

\subsection{New Production}

"New production" as defined by Eppley \& Peterson (1979; hereafter E\&P) represents the export of photosynthetically fixed carbon from the euphotic zone. This carbon flux is a vital part of the oceanic carbon cycle; it limits production at higher trophic levels (e.g. fish production) and supports life in the deep sea. It is also tightly linked to the oceanic cycles of oxygen and nitrogen and mediates the transport of many trace elements to the deep sea (Deuser et al., 1981).

\subsubsection{Classical Estimates}

Classical estimates of new production are based primarily on bottle incubations, where new production is defined as the portion of ${ }^{15} \mathrm{~N}$ uptake supplied by nitrate (Dugdale \& Goering, 1967). This fraction (the " $f$-ratio") is mulitiplied by the total carbon fixation rate (usually corrected for dark bottle effects, c.f. Laws et al., 1987) to yield a corresponding carbon flux. In the open ocean, new production measured in this fashion is typically a small fraction (10-20\%) of total primary production, and is estimated to account for about $2.5-10 \mathrm{gC} \mathrm{m}^{-2} \mathrm{yr}^{-1}$ (Platt \& Subba Rao, 1975; E\&P).

However, the measurement of new production in the oligotrophic open ocean has been problematic and controversial. Incubations may introduce artifacts due to trace metal contamination, confinement effects and heterotrophic respiration (Gieskes et al., 1979; Peterson, 
1980; McCarthy, 1984; Goldman \& Dennett, 1985; Li, 1987). Many questions remain as to what the bottle incubations really measure; variations related to the length of incubation, dark bottle effects and the light regime may contribute an uncertainty of 10-50\% (Harris, 1980). Long incubations are subject to species and abundance shifts, but short incubations make it difficult to extrapolate to daily productivity estimates. More recent measurements (Carpenter \& Lively, 1980; Fitzwater et al., 1982; Platt \& Harrison, 1986; Laws et al., 1987) suggest that such artifacts may have suppressed earlier estimates of primary productivity by a factor of 2-3, although the results are equivocal (Sharp et al., 1980; Marra \& Heinemann, 1984). Estimation of new production is further complicated by uncertainties in measuring the $f$-ratio contributed by additional nitrogen sources such as urea-N (up to $50 \%$ of recycled production; McCarthy \& Carpenter, 1983), nitrogen fixation (difficult to estimate; Martinez et al., 1983), and nitrification (may be important). Terrestrial and atmospheric sources can probably be ignored in the open ocean (Knap et al., 1986).

Another approach to measuring new production is to directly capture the sinking organic matter leaving the euphotic zone in sediment traps and measure its carbon and nitrogen content. However, it is difficult to derive quantitative results. The effective cross-section of a trap is difficult to determine, and may be dependent on particle size. Temporal trends may be aliased by advective effects and mesoscale features (Deuser, 1988). Short term deployments may be unrepresentative of long-term average conditions, while long term deployments may suffer from preservation artifacts. Shallow traps place near the bottom of the euphotic zone avoid the problem of decay while the particles are sinking, but artifacts due to "swimmers" are a significant problem (Knauer, 1984). In the northeast Pacific, the VERTEX group has attempted to derive quantitative new production estimates from sediment traps (Martin et al., 1987). These estimates are higher than the classical estimates, but still lower by a factor of 2-3 than the estimates from oxygen cycling discussed below.

\subsubsection{Tracer-based Estimates}

A serious challenge to classical new production estimates has come from studies of oxygen cycling, using both oxygen consumption in the main thermocline and the seasonal cycle of oxygen in the euphotic zone. These measurements of oxygen cycles offer a way to measure 
new production in situ without the need to perform incubations (which inevitably perturb the system under study). Furthermore, this approach tends to smooth out the small-scale variability that may bias bottle incubations.

However, this smoothing process destroys information about the details of new production and this approach can constrain only the gross magnitudes of the biological processes involved. The oxygen-based estimates are also somewhat model-dependent, and must account for the complex interplay of the many physical processes (e.g. barometric effects, radiative heating, mixing, air injection, gas exchange) that affect the oxygen concentrations. These uncertainties can be mitigated by simultaneous measurements of an analogous abiogenic tracer (e.g. ${ }^{3} \mathrm{He}$, Ar) but there is still some ambiguity in the choice of initial values and boundary conditions.

The rate of apparent oxygen utilization (AOUR) below the euphotic zone must be balanced by the oxidation of some reductant; the most likely candidate is organic matter (particulate and possibly also dissolved) sinking out of the euphotic zone. Jenkins has used ${ }^{3} \mathrm{H}-{ }^{3} \mathrm{He}$ as a "clock" to measure the AOUR in the main thermocline of the North Atlantic (Jenkins, 1977; $1980 ; 1982 \mathrm{a} ; 1987)$. This approach gives remarkably consistent results (see Figure 1-1) and suggests that AOUR decreases exponentially with depth in rough agreement with earlier work by Riley (1951) using AOU and geostrophic velocities and Packard et al. (1975) using electron transport activity to measure respiration rates. The depth-integrated AOUR below 100 meters is about 4-6 moles $\mathrm{O}_{2} \mathrm{~m}^{-2} \mathrm{yr}^{-1}$ corresponding to a new production carbon flux ${ }^{1}$ of $30-55 \mathrm{gC}$ $\mathrm{m}^{-2} \mathrm{yr}^{-1}$.

Schulenberger \& Reid (1981; hereafter S\&R) observed a summer subsurface maximum in oxygen concentration corresponding to a $20 \%$ supersaturation in the North Pacific and proposed that it was produced by photosynthetic production of oxygen. The rate required exceeded that derived from ${ }^{14} \mathrm{C}$-incubation measurements. Platt (1984a) has criticized their interpretation, pointing out that the time of oxygen accumulation is unknown, the initial conditions for temperature and oxygen are unknown, and the scatter in oxygen and ${ }^{14} \mathrm{C}$-incubation measurements is too large; he suggested that their estimate of oxygen production is not significantly different from that expected from the ${ }^{14} \mathrm{C}$-incubations. Reid \& Schulenberger (1986) have responded to Platt's criticism, arguing in favor of their original interpretation.

\footnotetext{
${ }^{1}$ This estimate is made using a Redfield ratio for $\mathrm{O}_{2}: \mathrm{C}$ of 1.3-1.7 (Takahashi et al., 1985).
} 


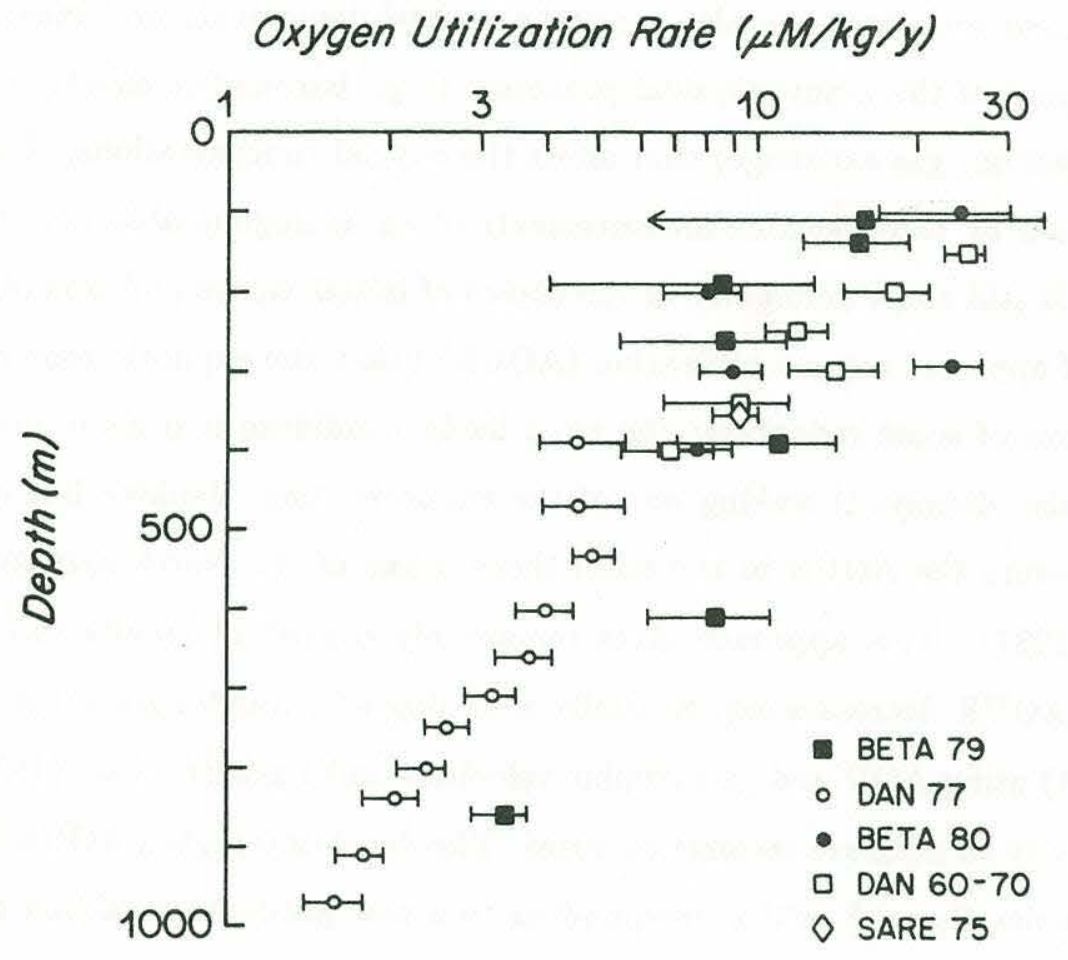

Figure 1-1: Measurements of apparent oxygen utilization rates (AOUR) vs. depth, from Jenkins (1987). 
However, all these authors miss a vital point: the $\mathrm{S} \& \mathrm{R}$ method measures new production whereas the ${ }^{14} \mathrm{C}$-incubations measure net production. The storage of oxygen in the euphotic zone over several months must represent production in excess of that which is recycled, i.e. new production. The incubations last $2-24 \mathrm{hr}$ and measure something close to net production (production in excess of photorespiration). The classical arguments suggest that new production is only $10-20 \%$ of net production in the oligotrophic ocean; therefore, the S\&R estimate is as much as an order of magnitude larger than expected from the incubation results.

However, these two approaches to measuring new production are not equivalent. As pointed out by Platt (1984a) and Platt \& Harrison (1985), classical productivity estimates are based on incubations that span timescales of hours whereas the S\&R method integrates productivity on timescales of months; since productivity is quite variable in space and time, the two methods are not directly comparable. Tracer-based estimates intrinsically integrate over large space and time scales, and hence will represent meaningful averages of possibly heterogeneous and sporadic biological processes. It may be inappropriate to extrapolate bottle incubation measurements to daily, annual, regional and global rates.

Moreover, the definition of new production developed by E\&P assumes that the euphotic zone is in steady-state with a relatively constant f-ratio. Platt \& Harrison (1985) have argued that the $f$-ratio may be dependent on the nitrate concentration and hence may vary seasonally. The positive correlation between total productivity and nitrate concentration would then lead to higher levels of new production; however, Goldman (1988) has pointed out that it is inappropriate to extrapolate their $f$-ratio vs. nitrate relationship to oligotrophic waters.

Nevertheless, the tracer-based estimates suggest that new production is much higher than traditionally thought. Why is there such a discrepancy? The answer may be related to the structure of the oligotrophic ecosystem (Jenkins \& Goldman, 1985; hereafter J\&G). If nutrient input to the euphotic zone is episodic, there may be two parallel, uncoupled food webs: a mature, highly developed one with many levels which is very efficient (low $f$-ratio), and an immature, inefficient one with few trophic levels which survives opporunistically on nutrient pulses (high $f$-ratio) (Frost, 1984; J\&G; Michaels \& Silver, 1988; Goldman, 1988). Perhaps the opportunistic system is associated with the maximum in $f$-ratio often observed near the base of the euphotic zone (Goering et al, 1970; Karl \& Knauer, 1984; Lewis et al., 1986; Altabet 
\& McCarthy, 1986; Coale \& Bruland, 1987). The response to these pulses would probably be short-lived (several hours; Goldman, 1988) and undersampled by the sparse coverage in time, location, and depth of traditional bottle casts; thus classical incubation techniques may undersample the latter system and hence miss much of the new production.

J\&G have used 20 years of hydrographic measurements at Station $S$ near Bermuda to show that the subsurface oxygen maximum is a seasonal phenomenon in the Sargasso Sea. They have documented the seasonal evolution of a photosynthetic oxygen maximum at 50 $\mathrm{m}$, and observed a counterpoint oxygen minimum due to oxidation below (Figure 1-2). J\&G estimate the magnitude of oxygen production by two methods. ${ }^{3} \mathrm{H}-{ }^{3} \mathrm{He}$ dating establishes a lower limit for the fraction of the gas inventory (oxygen and ${ }^{3} \mathrm{He}$ ) lost by mixing and gas exchange; this estimate applied to the observed inventories yields $5-6$ moles $\mathrm{O}_{2} \mathrm{~m}^{-2} \mathrm{yr}^{-1}$. A simple gas exchange calculation, using seasonally-modulated winds and wind-tunnel piston velocities, yields $5-10$ moles $\mathrm{O}_{2} \mathrm{~m}^{-2} \mathrm{yr}^{-1}$. The J\&G study confirms that new production rates in the open ocean are on the order of $50 \mathrm{gC} \mathrm{m}^{-2} \mathrm{yr}^{-1}$, indicating that either the $f$-ratio or total primary productivity must be much higher than previously thought.

The oxygen production in the euphotic zone is quantitatively balanced by the oxidation of 45 moles $\mathrm{O}_{2} \mathrm{~m}^{-2} \mathrm{yr}^{-1}$ below, and is also consistent with the magnitude of the counterpoint cycle due to respiration just below the euphotic zone. This balance is crucial, since it indicates that the deep oxygen consumption could be supported by local new production; if oxygen-depleted water or organic matter were imported laterally from areas of higher productivity (e.g. coastal or upwelling areas, as suggested by Martin et al., 1987), local oxygen production would be too small to support the consumption rates below.

J\&G conclude ${ }^{2}$ that new production in the Sargasso Sea is about $50-75 \mathrm{gC} \mathrm{m}^{-2} \mathrm{yr}^{-1}$; however, they admit that their estimates of gas exchange losses are too simplistic. Moreover, they require more direct evidence to account for physical processes that can produce apparent supersaturations (e.g. mixing, radiative heating, and bubble injection).

Musgrave et al. (1988) have attempted to refine the J\&G new production estimates by applying a one-dimensional upper ocean model to the seasonal cycle of oxygen and temperature at Station S. The temperature record is used to tightly constrain vertical mixing, and their

\footnotetext{
${ }^{2}$ They use a range for the photosynthetic quotient (PQ) $\mathrm{O}_{2}: \mathrm{C}$ of 1.0-1.8 (Williams et al., 1983).
} 


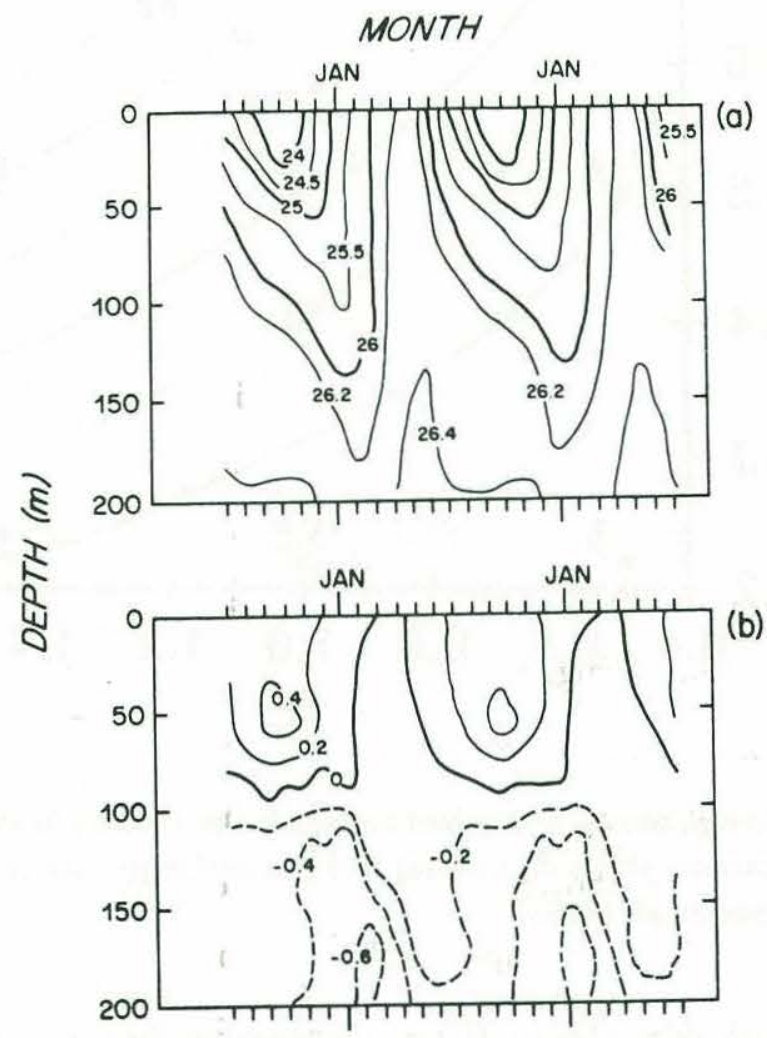

Figure 1-2: Seasonal cycles of (a) density and (b) oxygen anomaly at Station S, for the 1960-1970 average data, from Jenkins \& Goldman (1985). Oxygen anomalies are expressed as the difference between the measured concentration and the solubility, in $\mathrm{ml} / \mathrm{l}$. 


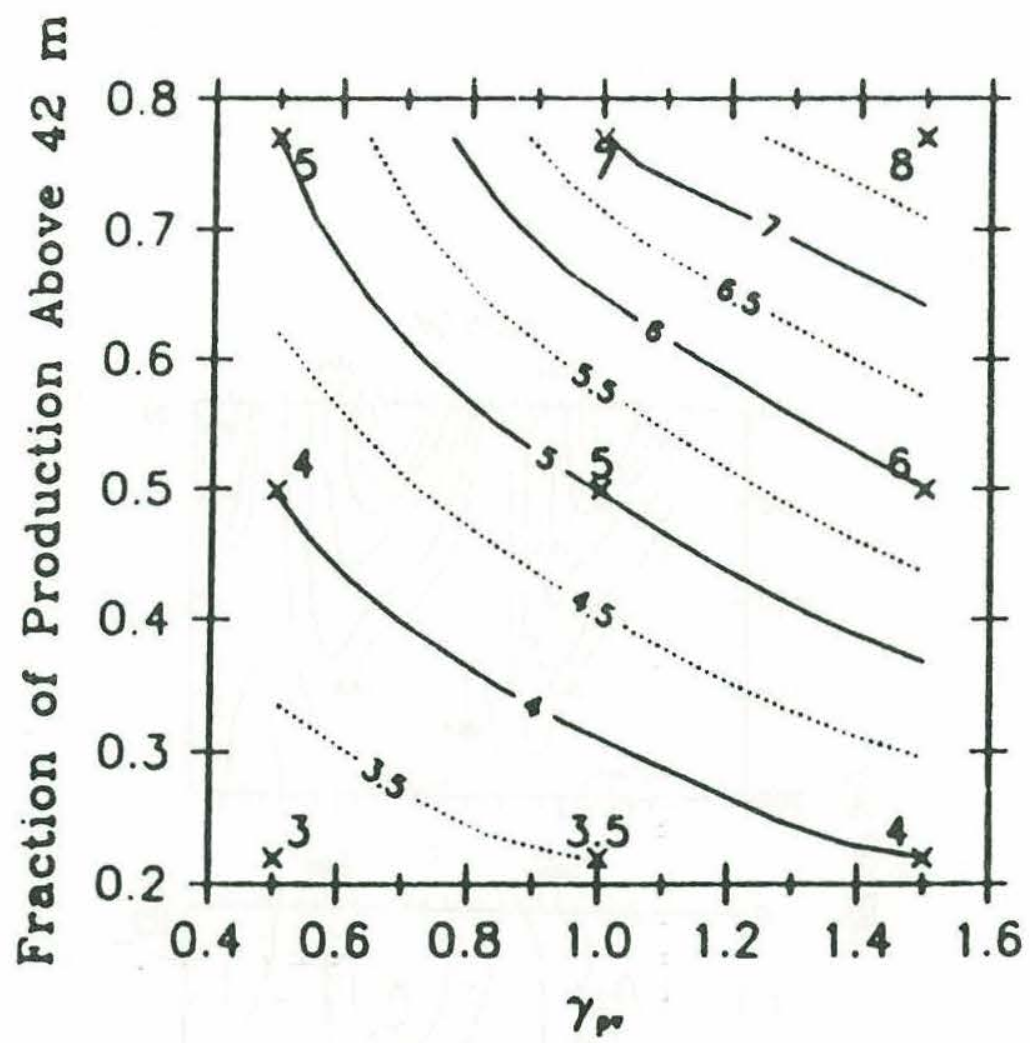

Figure 1-3: Biological oxygen production required to match the 1960-1970 average data as a function of the depth of production (fraction above 42 meters) and gas exchange rate (relative to a least-squares fit to field data), from Musgrave et al. (1988).

assumptions about the depth-dependence of new production lead to a conservative estimate. However, the precision of their production estimate is limited by uncertainty in the gas exchange rate and their results bracket the new production estimates obtained by J\&G (Figure 1-3).

Emerson (1988) has attempted to use a timeseries of hydrographic measurements from the subarctic Pacific (Station P) to put constraints on new production of oxygen. However, his estimates are based on mixed layer balances and ignore vertical mixing within the seasonal thermocline. He cannot demonstrate the closure of the annual cycle since his simplified calculations are appropriate only during the warming season. Thomas et al. (1989) have applied a more sophisticated mixed layer model which includes vertical mixing; however, uncertainties in the depth-dependence of production and in the gas exchange rate prevent a well-constrained estimate of oxygen production. Large et al. (1985) have demonstrated that advective effects 


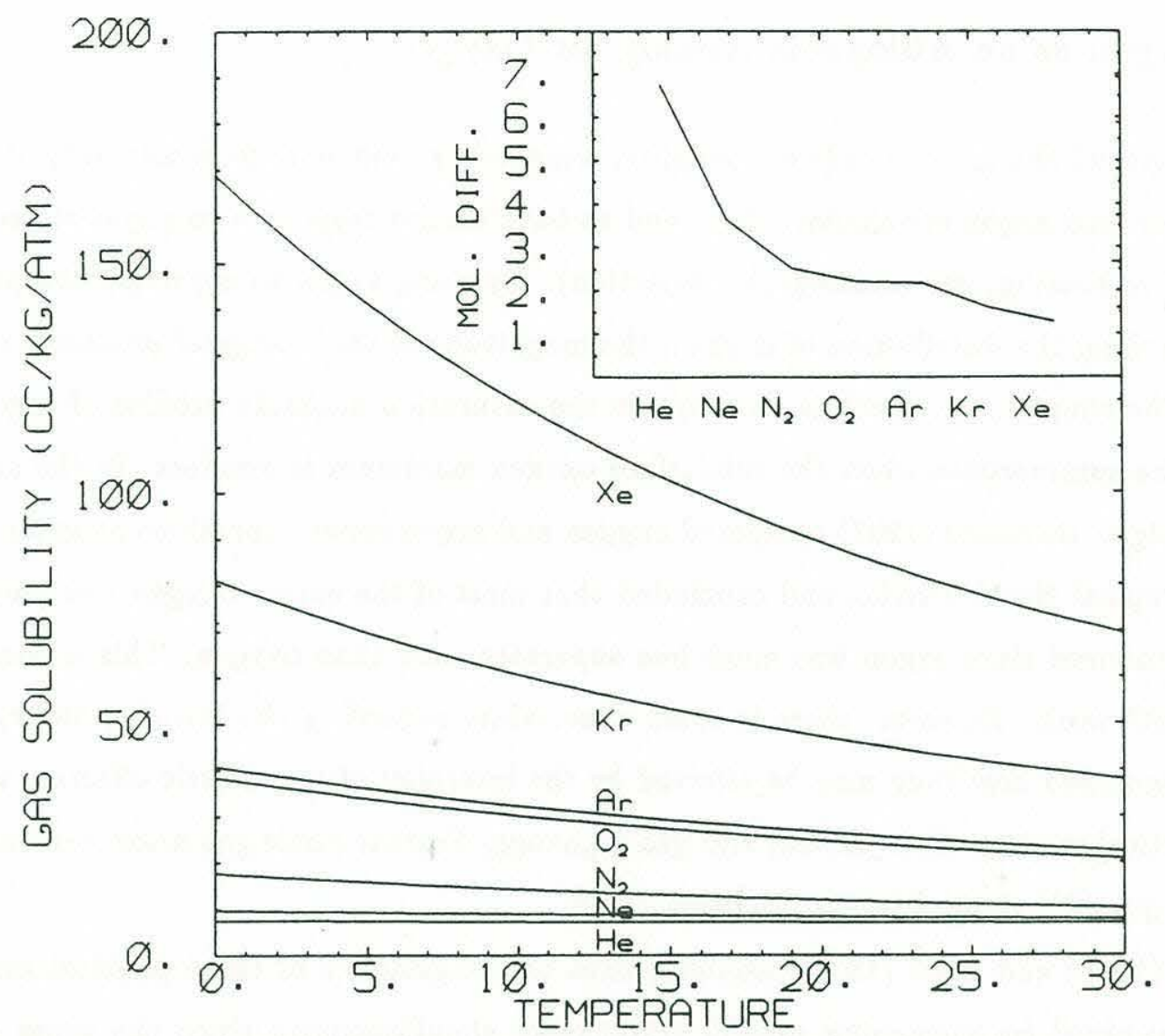

Figure 1-4: Physical properties of dissolved gases: solubilities vs. temperature, and molecular diffusivities at $20^{\circ} \mathrm{C}$ (inset). Solubilities are from Weiss (1970; 1971a; 1971b), Weiss \& Kyser (1978), and Weast (1982). Diffusivities are from Boerboom \& Kleyn (1969), Wise \& Houghton (1968), and Jahne et al. (1987).

may dominate the mixing of heat in the seasonal thermocline at Station $\mathrm{P}$, especially during the fall; thus, a vertical model may be inadequate here.

\subsection{Constraining Physical Processes with Noble Gases}

In order to use the distribution of oxygen to quantitatively estimate its biological production, it is necessary to account for the physical processes affecting oxygen. A natural way to do this is to use the noble gases as tracers. The noble gases ( $\mathrm{He}, \mathrm{Ne}, \mathrm{Ar}, \mathrm{Kr}, \mathrm{Xe}, \mathrm{Rn}$ ) are well-suited for studying physical processes because they are biologically and chemically inert and their physical properties are well known and span a wide range (Figure 1-4). 


\subsubsection{Argon as an Abiogenic Analog for Oxygen}

Since the physical characteristics (i.e. solubility, temperature dependence of solubility, diffusivity) of oxygen and argon are similar, they tend to have similar responses to physical processes (e.g. radiative heating, gas exchange, air injection). By using argon to constrain the physical effects controlling the distribution of oxygen, the magnitude of the biological processes may be deduced. The simplest approach is to compare the saturation anomaly profiles of argon and oxygen in the summertime when the subsurface oxygen maximum is greatest. In the summer of 1985, Craig \& Hayward (1987) compared oxygen and argon supersaturations at two stations in the subtropical North Pacific, and concluded that most of the excess oxygen was photosynthetically produced since argon was much less supersaturated than oxygen. This is consistent with the J\&G work. However, there is some uncertainty regarding the initial (winter) argon concentrations, and how they may be affected by the interplay of barometric effects, radiative heating, vertical mixing, air injection, and gas exchange. Further noble gas measurements offer a means to quantify these processes in more detail.

Benson (1965) and Bieri (1974) proposed that the magnitudes of these physical processes can be determined by measuring several noble gases simultaneously since the gases will be fractionated to varying degrees. An equation can be written for each gas and the resulting linear system can be solved to estimate the magnitude of each effect ${ }^{3}$. To apply this technique successfully, the gas concentrations and solubilities must be known to an accuracy of better than $1 \%$. At the time of Bieri's review, this accuracy was not attainable in practice; today, noble gas determinations can be made to order 0.1-0.3\% (Lott and Jenkins, 1984; Craig and Hayward, 1987; Spitzer \& Jenkins, 1989). However, these equations are incomplete, since they assume steady-state, ignore gas exchange, and assume that the various effects combine linearly. A more sophisticated numerical model can account for these complexities.

\footnotetext{
${ }^{3}$ Bieri accounted for these effects as follows:

$$
\Delta_{i}=\varepsilon-\left(\frac{1}{\beta_{i}} \frac{\partial \beta_{i}}{\partial T}\right) \Delta T+\nu / \beta_{i}
$$

where $\varepsilon$ is the fractional variation in barometric pressure from one atmosphere, $\beta_{i}$ is the Bunsen solubility coefficient of the gas, $T$ is the temperature, and $\nu$ is the volume (in cc STP) of air injected.
} 


\subsubsection{Sources of Saturation Anomalies}

Although noble gas concentrations tend to be close to saturation values, small departures from saturation provide clues to physical processes at work. Saturation anomalies can be defined as the percent difference of the gas concentration from its solubility value:

$$
\Delta_{i}=\left(C_{i} / C_{i}^{*}-1\right) \times 100 \%
$$

where $C_{i}$ is the in-situ gas concentration and $C_{i}^{*}$ is the normal atmospheric equilibrium concentration of the gas (the product of the partial pressure of the gas and its Bunsen solubility coefficient, $\beta_{i}$ ). Sources of saturation anomalies include equilibration of gases at different barometric pressures, radiative heating, mixing, and air injection by bubbles.

Barometric effects produce identical saturation anomalies for all the noble gases, but radiative heating or cooling will produce larger saturation anomalies for the heavier noble gases because of their larger solubility dependence on temperature (Figure 1-4). Mixing between water masses of different temperature and salinity (and hence different gas solubilities) can produce supersaturation anomalies because of the positive curvature of the solubility-temperature relationship (Figure 1-4) ${ }^{4}$.

Bubbles may affect gas exchange, especially for the less soluble gases. Laboratory and theoretical work suggests that bubbles may significantly enhance gas exchange at wind speeds above about $10 \mathrm{~m} \mathrm{~s}^{-1}$ (Atkinson, 1973; Merlivat \& Memery, 1983; Broecker \& Siems, 1984; Thorpe, 1984c). However, recent work by Thorpe (1986) suggests that bubbles may be important at lower wind speeds as well. Bieri (1974) used a simple model for air injection; it assumes that bubbles of atmospheric air are injected beneath the water by breaking waves where they are forced into solution by hydrostatic pressure. If the bubbles dissolve completely, this process will preferentially enrich surface water in the less soluble gases (e.g. He). Other processes, such as partial dissolution of bubbles, may compete with simple air injection and introduce more complex fractionation effects.

Fuchs et al. (1986) have proposed three modes of air injection dependent on bubble size.

\footnotetext{
${ }^{4}$ This curvature is larger for the heavier noble gases; however, the predicted effect is usually less than $0.1 \%$ even for krypton.
} 
Small bubbles dissolve completely, whereas bubbles of intermediate size reach solubility equilibrium with the surrounding water. Gas loss from large bubbles is diffusively-limited. The dynamic balance between these bubble processes and gas exchange is represented as a linear combination of these three processes:

$$
f_{i} \alpha_{i} w_{i} x_{i}=\left(w^{i n j}+\left(w^{e x c h}+\alpha w_{i}^{\text {diff }}\right)\left(p^{\prime} / p_{0}-f_{i}\right)\right) x_{i}
$$

with transfer coefficients representing the fluxes of bubbles that dissolve completely $\left(w_{\text {inj }}\right)$, approach solubility equilibrium with the water $\left(w^{\text {exch }}\right)$, and exchange diffusively with the surrounding water $\left(w_{i}^{\text {diff }}\right)$. The transfer coefficient for gas exchange is $w_{i}, \alpha$ is the Ostwald gas solubility, $x_{i}$ is the atmospheric abundance of the gas, $f_{i}$ is its fractional supersaturation, and $p^{\prime} / p_{0}$ is the average pressure excess in the bubbles relative to atmospheric pressure. On the basis of literature measurements of noble gas supersaturations, they estimate that $w^{\text {exch }}$ can probably be neglected.

Jenkins (1988) has adopted a similar approach, focussing on the same two modes as "endmembers" of the air injection process. He incorporates a cubic dependence of the air injection rate on the wind speed, in accordance with observations by Crawford \& Farmer (1986). On the basis of GEOSECS measurements of helium and neon supersaturations, he estimates that "partial trapping" (diffusively limited gas loss from bubbles) dominates the air injection process, contributing $63 \pm 13 \%$.

\subsubsection{Gas Exchange}

Gas exchange competes with the above processes within the mixed layer by driving gas concentrations toward their equilibrium values; due to the finite impedance of this process, solubility equilibrium is often incompletely attained. Although gas exchange has been the subject of extensive laboratory, field and theoretical research (Liss, 1983; Roether, 1985; Liss \& Merlivat, 1986) we have only a crude understanding of the dependence of gas exchange rates in realistic oceanic conditions on environmental parameters.

The gas flux is determined by the difference between the atmospheric equilibrium concentration $\left(C_{i}^{*}\right)$ and the concentration of the gas in the bulk liquid $\left(C_{i}\right)$, multiplied by a transfer 
coefficient $\left(k_{w}\right)$ :

$$
F=k_{w}\left(C_{i}^{*}-C_{i}\right)
$$

For unreactive, slightly soluble gases (e.g. noble gases, $\mathrm{O}_{2}, \mathrm{~N}_{2}$ ), the gas transfer coefficient across the interface $\left(k_{w}\right)$ is mainly determined by the impedance to exchange presented by the boundary layer in the liquid phase; for soluble gases $\left(e . g . \mathrm{CO}_{2}\right)$, the impedance is in the gas phase (Liss, 1983).

The magnitude of the transfer coefficent $k_{w}$ depends both on the physical properties of the gas and the environmental conditions (e.g. wind speed, sea state, waves, bubbles, surface films). Transfer rates are proportional to a positive power of the Schmidt number, $S c=\nu / D$, the ratio of kinematic viscosity to molecular diffusivity (Roether, 1985; Liss and Merlivat, 1986). Since the lighter gases have higher diffusion constants (Figure 1-4), their exchange rates are faster. Since diffusivities increase with temperature, gas transfer rates will increase also; however, this effect is somewhat offset by a correlation between lower temperatures and higher winds in field conditions.

The classic model for gas exchange depicts the air-sea interface as a double laminar boundary layer through which gases diffuse between the bulk liquid and gas phases (Liss, 1983). This model predicts that exchange rates vary directly with the gas diffusivity. A slightly different model assumes that gas transfer is limited by the rate of replacement of a stagnant film at the surface; in this case, the transfer coefficient is proportional to $D^{1 / 2}$. Deacon (1977) has derived an analytical expression for the transfer coefficient by treating the air-sea interface as a smooth, rigid wall and predicts a $S c^{-2 / 3}$ dependence. More sophisticated theoretical approaches to gas exchange have been pursued (Coantic, 1986; Liss, 1983); however, the simple boundary layer model is adequate to interpret most of the field data.

Field measurements of gas exchange rates are based on a variety of methods, reviewed by Broecker (1982), Liss (1983), and Roether (1985) spanning time scales from about 5 days $\left({ }^{222} \mathrm{Rn}\right.$ method) to about 2 years (global ${ }^{14} \mathrm{C}$ balance). A major deficiency in the field data is its seasonal bias; most measurements have been carried out in summer, with few exceptions (e.g. Peng, et al., 1974; Tsunogai \& Tonaka, 1980). The field data suggest that at wind speeds less than $5 \mathrm{~m} \mathrm{~s}^{-1}, k_{w} \propto S c^{-2 / 3}$ in agreement with Deacon's model. However, at high wind speeds, $k_{w} \propto S c^{-1 / 2}$ in accordance with the film replacement model. Liss \& Merlivat (1986) 


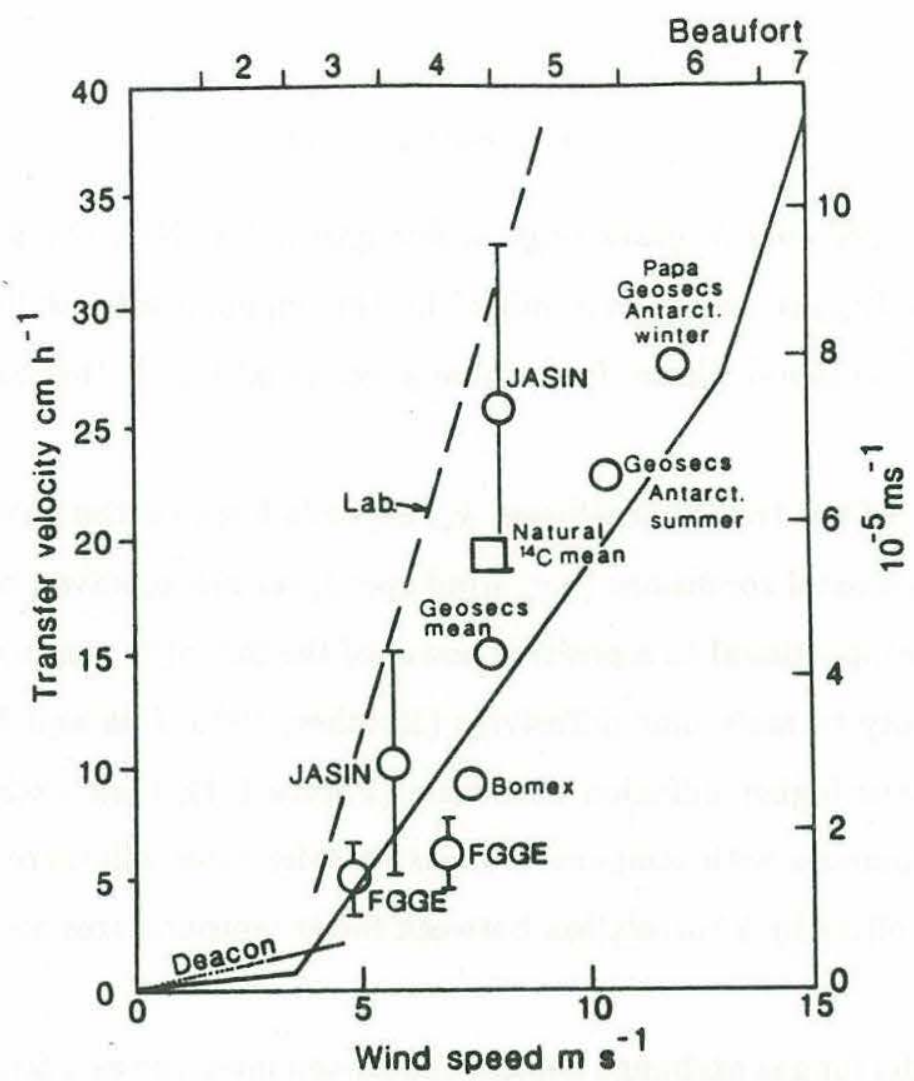

Figure 1-5: Gas transfer rates: piston velocity vs. wind speed (at 10 meter height), from Liss \& Merlivat (1986). The dotted line is a prediction based on Deacon's (1977) smooth surface model. The dashed line represents laboratory wind tunnel results. The solid lines are fits proposed by Liss \& Merlivat. All transfer rates are normalized to $\mathrm{Sc}=600$, corresponding to $\mathrm{CO}_{2}$ at $20^{\circ} \mathrm{C}$.

combine field data with wind tunnel measurements and theoretical considerations to estimate a new piston velocity vs. wind speed relationship (Figure 1-5), incorporating enhanced exchange rates at higher wind speeds. A new method using an eddy-correlation technique offers promise in measuring gas exchange rates for soluble gases (with appreciable gas phase resistance); however, it has been applied only in near-shore environments, where it indicates very high exchange rates (Wesley et al., 1982; Smith \& Jones, 1985) possibly due to enhanced exchange rates in this high energy environment.

The effect of surface films is probably most important at low wind speeds and in polluted waters; it is probably unimportant in the open ocean although field measurements are scarce. Goldman's (1988) results suggest that surfactants can reduce exchange rates by up to $50 \%$. 
However, gas exchange rates in Sargasso Sea water are only about $5 \%$ lower than in clean distilled water.

\subsection{Supplying the Upward Flux of Nutrients}

If new production is as large as suggested by the oxygen studies, an additional paradox arises: How can enough nutrients be supplied to the euphotic zone to support this production? Classical estimates based on vertical advection-diffusion models (with vertical diffusivities of order $1 \times$ $10^{-4} \mathrm{~m}^{2} \mathrm{~s}^{-1}$ ) can account for only about $10 \%$ of the required flux. Estimates of vertical turbulent diffusivities based on microstructure suggest values 2-10 times even lower (Garett, 1979; Schmitt, 1981; Gargett, 1984; Lewis et al., 1986).

Yet, Jenkins (1988b) has used the distribution of helium isotopes to argue that the nutrient flux from the main thermocline is sufficient to support the high levels of new production required by the oxygen cycles. The $\delta^{3} \mathrm{He}$ of surface water ${ }^{5}$ is controlled by the isotope effect on solution $\left({ }^{3} \mathrm{He}\right.$ is about $1.7 \%$ o less soluble than $\left.{ }^{4} \mathrm{He}\right)$, in situ ${ }^{3} \mathrm{He}$ production from ${ }^{3} \mathrm{H}$ decay, mixing of ${ }^{3} \mathrm{He}$ rich waters from below, and bubble injection (at or near the atmospheric ratio).

The ${ }^{3} \mathrm{H}-{ }^{3} \mathrm{He}$ age ${ }^{6}$ of the mixed layer is essentially zero ( $\pm 0.3 \mathrm{yr}$; Fuchs et al., 1986), indicating that helium is quickly lost to the atmosphere so that no excess ${ }^{3} \mathrm{He}$ can build up (Jenkins, $1980 ; 1987 ; \mathrm{J} \& \mathrm{G}$ ). Although air injection tends to lower the $\delta^{3} \mathrm{He}$ of the mixed layer (by up to $2 \%$; Fuchs et al., 1986), this effect is offset by the flux of tritiugenic ${ }^{3} \mathrm{He}$ from below. Jenkins $(1988 \mathrm{a} ; 1988 \mathrm{~b})$ treats the dynamic balance among these processes with a one-dimensional upper ocean model. The observed ${ }^{3} \mathrm{He}$ excess in the mixed layer implies an annual outgassing flux of ${ }^{3} \mathrm{He}$ that is consistent with the temporal evolution of the ${ }^{3} \mathrm{H}-{ }^{3} \mathrm{He}$ inventory in the main thermocline. Furthermore, the flux of ${ }^{3} \mathrm{He}$ can be used to estimate the flux of nitrate, since the distributions of the two tracers are well-correlated in the main thermocline. On this basis,

\footnotetext{
${ }^{5} \mathrm{The} \delta^{3} \mathrm{He}$ is defined as the per mil difference between the ${ }^{3} \mathrm{He} /{ }^{4} \mathrm{He}$ of a sample compared with the atmospheric ratio:$$
\delta^{3} \mathrm{He}=\left[\left({ }^{3} \mathrm{He} /{ }^{4} \mathrm{He}\right)_{x} /\left({ }^{3} \mathrm{He} /{ }^{4} \mathrm{He}\right)_{a t m}-1\right] \times 1000 \%
$$

${ }^{6} \mathrm{The}{ }^{3} \mathrm{H}-{ }^{3} \mathrm{He}$ age is defined as:

$$
\lambda^{-1} \log \left(1+\left[{ }^{3} \mathrm{He}\right] /\left[{ }^{3} \mathrm{H}\right]\right)
$$

where $\lambda$ is the radioactive decay rate $\left({ }^{3} \mathrm{H}\right.$ has a half-life of 12.4 years $)$.
} 
Jenkins (1988b) argues that the nitrate flux is consistent with oxygen-based estimates of new production.

The mechanism by which the nutrients are supplied to surface waters may be related to isopycnal processes. Jenkins (1980) has shown for tritium and its radiogenic daughter ${ }^{3} \mathrm{He}$ that isopycnal processes dominate diapycnal processes in determining its distribution in the main thermocline; this is likely the case for nitrate as well, since its distribution and gradients are similar.

Hamilton et al. (1989) have suggested that salt fingering may be responsible for enhancing vertical tubulent fluxes of nitrate (by a factor of 6) and heat (by a factor of 3) relative to estimates based on microstructure. They argue that this enhancement might provide enough nitrate to fuel the measured levels of oxygen production. However, the confidence limits on the microstructure measurements span several orders of magnitude and it is difficult to quantify the implications of this hypothesis. Even if the salt fingering hypothesis is true, another mechanism must be sought to explain the high levels of new production observed in the Pacific where salt fingering does not occur.

Another possibility is that the extra nutrients may be supplied by some form of nutrient pulsing. J\&G suggested that a turbulent mechanism such as that proposed by Klein \& Coste (1984) might mix pulses of nutrients into the euphotic zone; however, the penetration depth of this process is too shallow to reach the nutricline in the Sargasso Sea (Musgrave et al., 1988). Storms may cause deepening of the mixed layer and enhanced mixing; however, effects of storms (except during late winter) do not usually penetrate deep enough to bring nutrients into the euphotic zone. Alternatively, mesoscale eddies may enhance nutrient fluxes to the upper layers as observed for warm core rings (Nelson et al., 1989, Joyce, 1988). Jenkins (1988b) argues that he observed such a nutrient injection event at Station S in July, 1986 and I will explore this hypothesis further in Chapter 4.

\subsection{Research Strategy}

I have undertaken a three year study (April 1985 - April 1988) at Station $\mathrm{S}\left(32^{\circ} \mathrm{N} 64^{\circ} \mathrm{W}\right)$, situated 25 kilometers southeast of Bermuda in the Sargasso Sea (see Figure 1-6) in $2700 \mathrm{~m}$ water depth. I have augmented an ongoing series of biweekly hydrographic stations which measure 


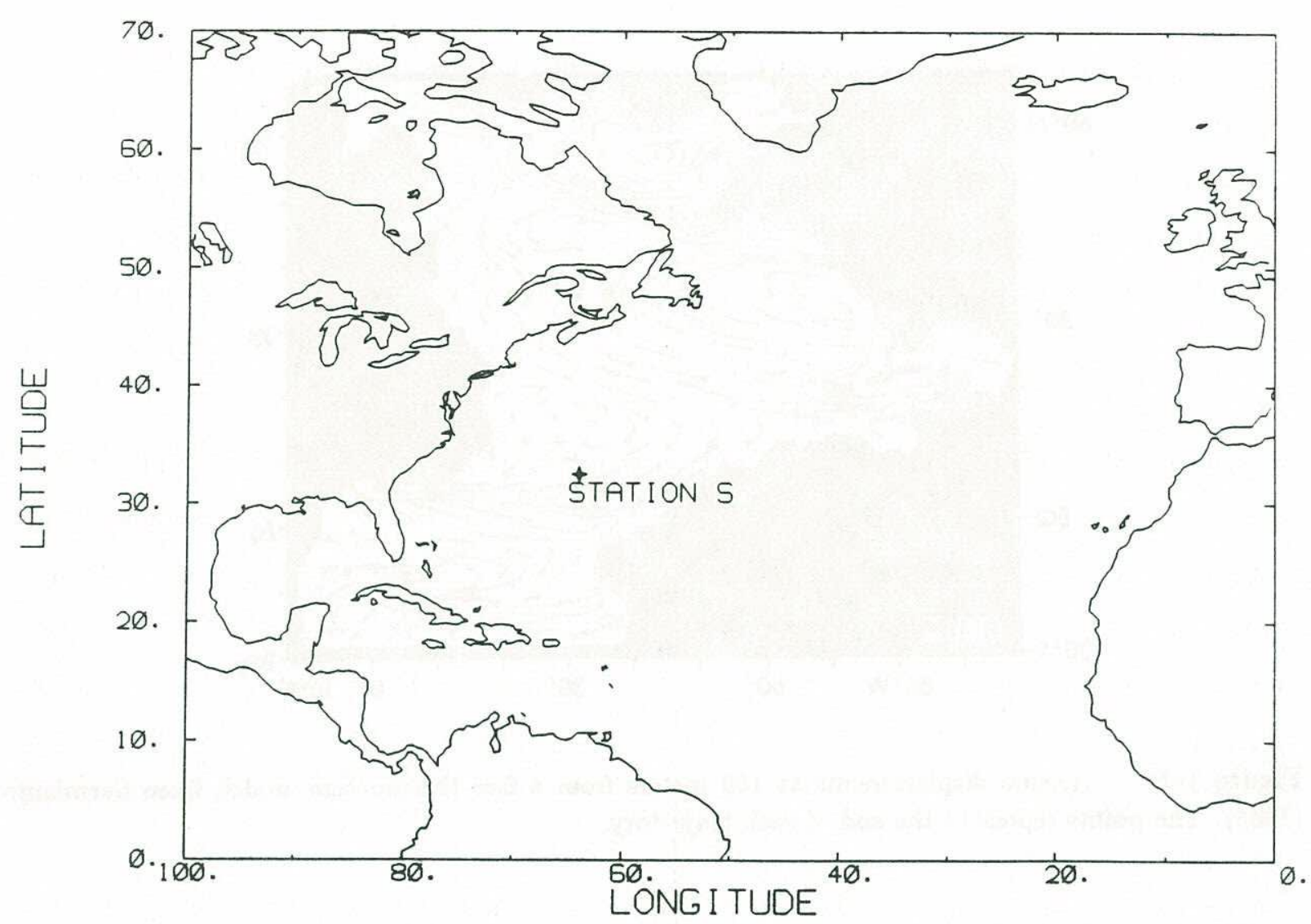

Figure 1-6: Location of sampling site.

temperature, salinity and oxygen at standard depths with approximately monthly sampling for nutrients, argon, helium, ${ }^{3} \mathrm{H} /{ }^{3} \mathrm{He}$ and an additional shallow cast to obtain approximately 10 meter resolution in the upper 100 meters.

There are several reasons for choosing the Station S site. Logistics are greatly simplified since the Bermuda Biological Station (BBS) can routinely collect samples and perform oxygen and nutrient analyses as an adjunct to biweekly hydrographic stations. Furthermore, the 35 year (1954-present) hydrographic record (WHOI \& BBSR, 1988) provides a context in which to interpret the results of the 3 year study. Seasonal and interannual variations in productivity have been documented by Menzel \& Ryther $(1960 ; 1961)$ and at the nearby OFP site by Deuser (1986; 1988). J\&G have observed interannual modulation of the seasonal oxygen cycle in the upper 100 meters. These results suggest that seasonal variations must be examined in light of interannual trends. 


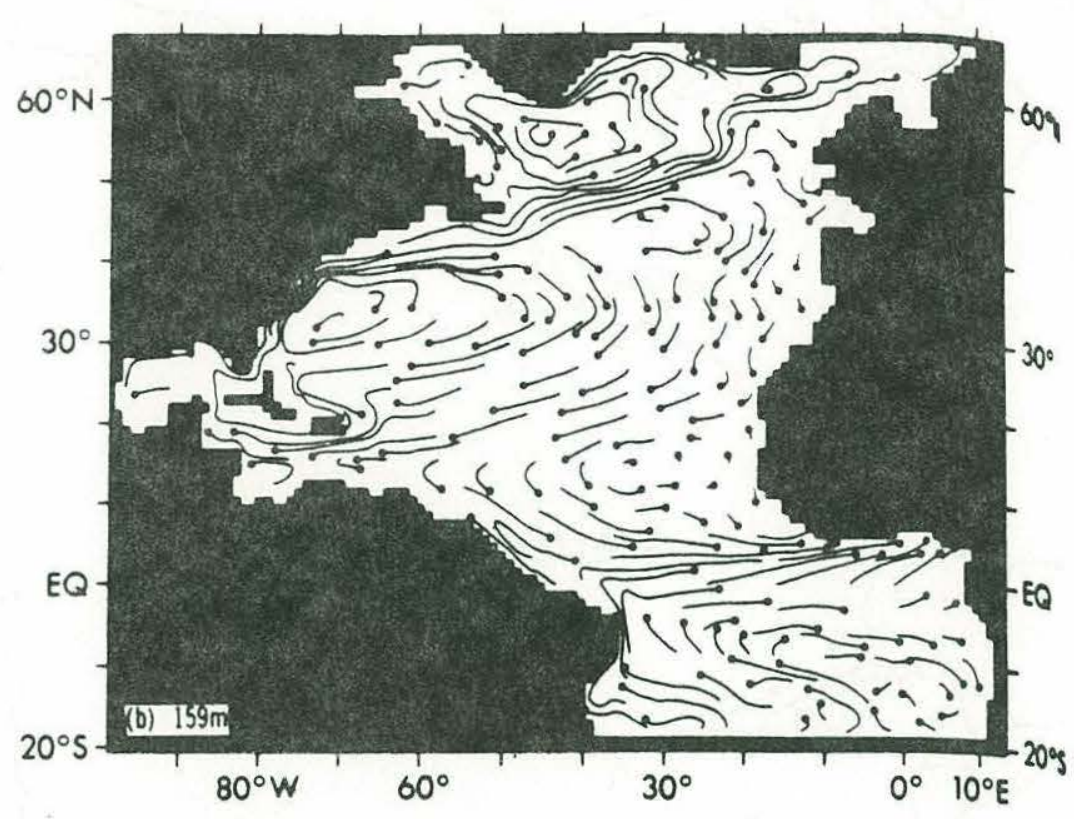

Figure 1-7: Annual displacements at 159 meters from a free thermocline model, from Sarmiento (1983). The points represent the end of each trajectory.

The site is within the tight recirculation region of the N. Atlantic subtropical gyre (Worthington, 1976) so that tracer distributions should represent an average over a large area (Figure 1-7. Also, as shown by Woods \& Barkmann (1986), the net annual heat flux at this site is nearly zero (Figure 1-8). Moreover, the water advected past the site by the mean flow also tends to originate near regions with small net annual heat flux so that one would expect closure for annual cycles. As indicated by the observed seasonal trends, aliasing of the seasonal cycle by advected features is not severe.

Although Station S is near Bermuda, Menzel \& Ryther (1960) have observed no "island effect" on productivity. Hogg (1978) attributed enhanced fine structure in temperature on the W and NW side of the island to dynamic shear flow instability. He also observed two cold core Gulf Stream rings in the vicinity. However, Frankignoul (1981) found significant mesoscale eddy activity but minimal evidence of the passage of cold core rings (1-3 rings) in analyzing the hydrographic data from Station S during 1954-1978. Wunsch (1983) has suggested that this may be due to the fact that the island of Bermuda "shields" Station S from cold core rings coming from the north and east. 


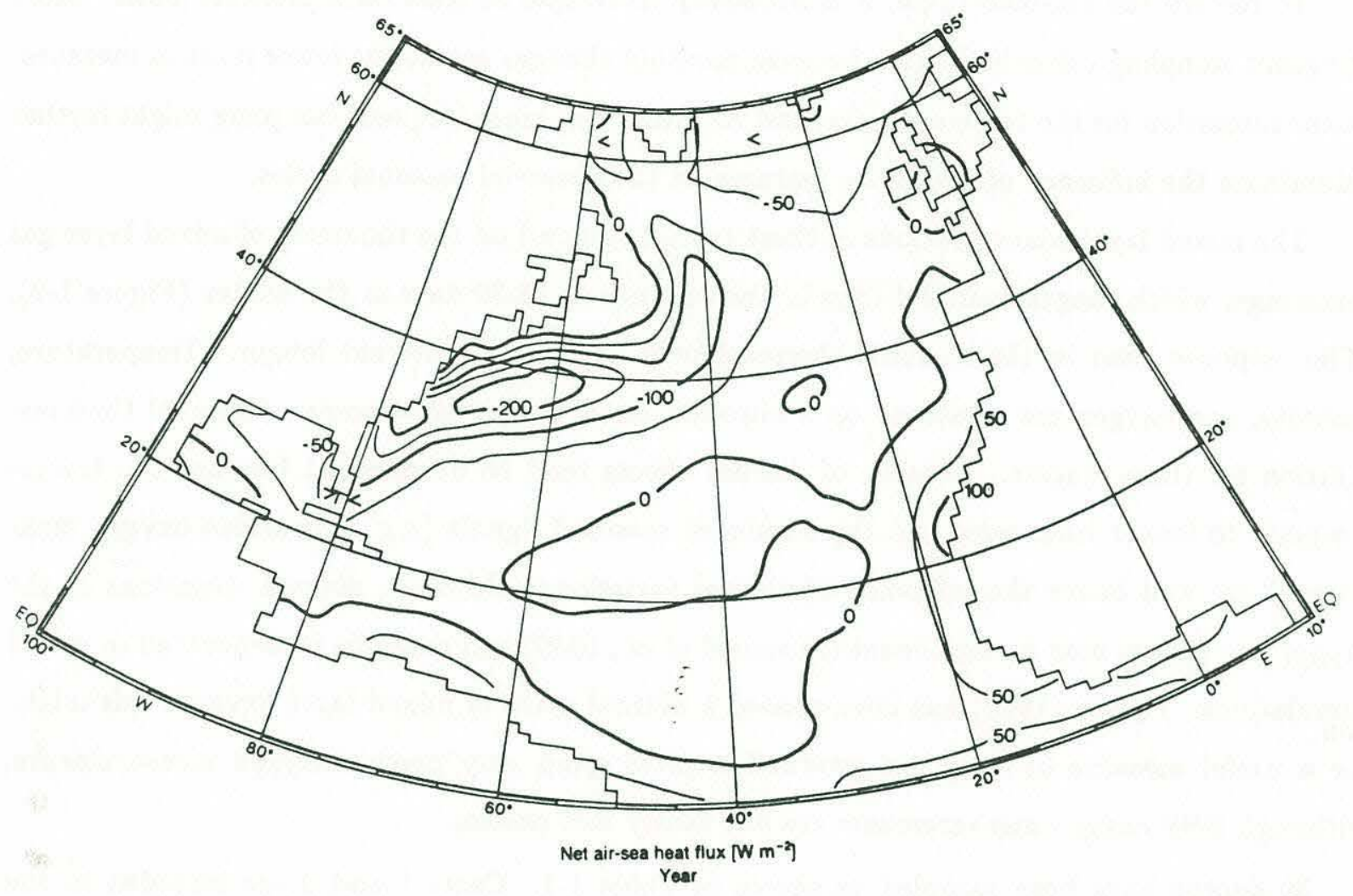

Figure 1-8: Net annual heat flux in the North Atlantic, from Isemer \& Hasse (1985). 
Nevertheless, the three year duration is a minimum for resolving the seasonal cycle from the influence of eddies and rings (Frankignoul, 1981). Fortunately, the similarities of the tracers tend to minimize aliasing of eddies and interannual trends, and it should be possible to evaluate the short-term balance of oxygen and nutrient fluxes.

To resolve the seasonal cycle, it is necessary to sample at least on a monthly basis. More frequent sampling offers little gain because monthly changes are at the lower limit of measurement resolution for the tracers $\mathrm{He}, \mathrm{O}_{2}$, and Ar. However, more frequent sampling might further illuminate the influence of mesoscale features on the observed seasonal cycles.

The mixed layer concentrations of these tracers respond on the timescale of mixed layer gas exchange, which ranges from 2-4 days in the summer to 15-30 days in the winter (Figure 1-9). The response time in the seasonal thermocline is probably somewhat longer. Temperature, salinity, and oxygen are measured on a biweekly basis, so this gives some additional time resolution for these tracers. Aliasing of diurnal effects tend to be minimal because the tracers respond to longer timescales and the dominant seasonal signals (e.g. subsurface oxygen maximum) are well below the influence of diurnal variations. However, diurnal variations in the upper few meters may be significant (Stommel et al., 1969) and could be incorporated in model simulations. Tijssen (1979) has documented a diurnal cycle in mixed layer oxygen; this might be a useful measure of daily net production rates given very precise oxygen measurements, although BBS oxygen measurements are not nearly this precise.

39 depths have been sampled as shown in Table 1-1. Casts 1 and 2 are included in the biweekly hydrostations; Cast 3 is added to increase vertical resolution in the upper $200 \mathrm{~m}$ where the seasonal cycle is most pronounced. The goal is to have 4 bottles in the mixed layer to obtain precise mixed layer averages, 5 in the seasonal thermocline to resolve the subsurface oxygen maximum, and a set of standard depths to document deeper cycles. XBT's are dropped between casts and after the last cast to detect potential aliasing by internal waves, diurnal changes and ship drift during sampling. Once the Niskin bottle is vented, the argon sample is drawn first, followed by the helium sample. The water used to flush the helium sampler is then used to collect an oxygen sample; Jenkins (pers. comm.) has shown that this protocol does not measurably affect the oxygen sample. Finally, nutrient and salt samples are drawn.

The expected seasonal variation in argon concentration at Station S is about $15 \%$, and the 


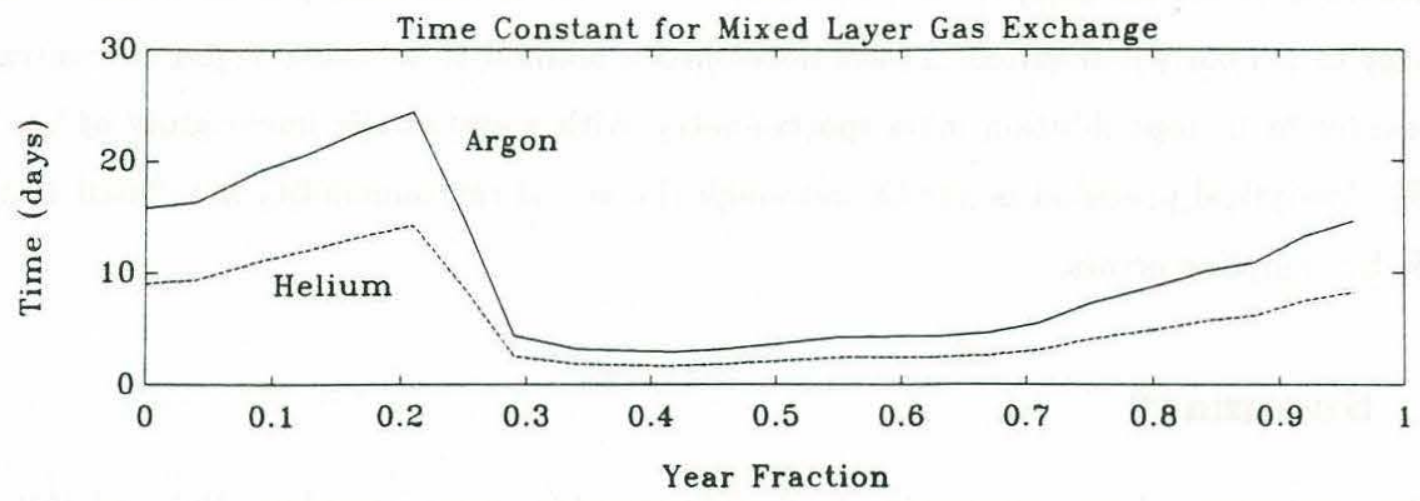

Figure 1-9: Time constants for mixed layer gas exchange for argon (solid line) and helium (dotted line) computed as a function of time of year, using climatological wind speeds (Isemer \& Hasse, 1985) and Liss \& Merlivat (1986) piston velocities.

\begin{tabular}{rrr}
\hline Cast 1 & Cast 2 & Cast 3 \\
\hline 600 & 1 & 1 \\
700 & 10 & 10 \\
800 & 25 & 20 \\
900 & 50 & 30 \\
1000 & 75 & 40 \\
1200 & 100 & 50 \\
1400 & 150 & 60 \\
1600 & 200 & 70 \\
1800 & 250 & 80 \\
2000 & 300 & 90 \\
2200 & 350 & 125 \\
2400 & 400 & 150 \\
2600 & 500 & 175 \\
\hline
\end{tabular}

Table 1-1: Nominal sampling depths (meters). 
maximum observed saturation anomalies are about $3-5 \%$. To determine whether the summer oxygen maximum is a result of physical or biological processes, the argon concentration must be measured to an accuracy of about $1-2 \%$. However, to estimate gas exchange rates, an accuracy of $0.3-0.5 \%$ is required. I have developed a method to measure argon concentrations in seawater by isotope dilution mass spectrometry, with a systematic uncertainty of less than $\pm 0.2 \%$. Analytical precision is $\pm 0.3 \%$, although the actual reproducibility is inflated to about $\pm 0.6 \%$ by sampling errors.

\subsection{Summary}

Classical estimates of new production in the oligotrophic ocean, based on ${ }^{14} \mathrm{C}$ and ${ }^{15} \mathrm{~N}$ incubations, suggest that it is only a small fraction of total primary production. Estimates based on oxygen cycling (respiration rates in the main thermocline, accumulation of oxygen in the seasonal thermocline) suggest that new production may be an order of magnitude higher. By simultaneously making noble gas measurements, the physical processes affecting the oxygen distribution can be accounted for more precisely. Craig \& Hayward (1987) demonstrated that argon serves as a good abiogenic analog for oxygen at a site in the subtropical N. Pacific. Work by Bieri and Benson demonstrates the potential for using several noble gases with different properties to understand several physical processes simultaneously. Chou (1985) and Musgrave et al. (1988) applied a one-dimensional upper ocean model to the seasonal cycle of oxygen and temperature to put constraints on oxygen production at Station S near Bermuda.

I will extend these efforts by making measurements of the seasonal cycles of noble gases and oxygen at Station S. Argon will be used as an inert analog for physical processes affecting oxygen. Measurements of helium and its isotopes will be used to further constrain the mass balances. By using the seasonal cycles of thermal and wind forcing, I attempt to put constraints on the mechanisms and rates of physical processes affecting gases in the upper ocean, and to understand their dependence on this forcing. I combine the noble gas and oxygen data with an upper ocean model to place quantitative constraints on both the physical processes and the biological production of oxygen.

Finally, I attempt to understand the nitrate flux to the euphotic zone. Jenkins has shown that this flux is consistent with oxygen-based new production estimates. However, rates of 
apparent vertical mixing consistent with the noble gas, oxygen, and temperature cycles are much too small to satisfy this flux. I explore the hypothesis that episodic injections of nutrients may provide a large portion of the required flux. 


\section{Chapter 2}

\section{Methods}

\subsection{Introduction}

The analytical methods used in making the timeseries measurements at Station S are discussed below. We have developed an analytical technique to measure argon concentrations in seawater by isotope dilution mass spectrometry, with a systematic uncertainty less than $\pm 0.2 \%$. Analytical precision is $\pm 0.3 \%$, although reproducibility of samples from Station $\mathrm{S}$ is inflated to approximately $\pm 0.6 \%$ by errors associated with sampling. We use the methods of Jenkins (1987) to measure helium isotopes by mass spectrometry. Hydrographic measurements (temperature, salinity, oxygen, nutrients) are carried out at the Bermuda Biological Station using standard methods, and their uncertainty is assessed in the context of the historical record at Station S.

\subsection{Argon Measurements in Seawater}

Most noble gas measurements are made by isotope dilution mass spectrometry (IDMS) (Bieri, 1974; Kester 1975); however, argon has been measured by gas chromatography (GC) as well (Weiss \& Craig, 1973; Brettel, 1985; Craig \& Hayward, 1987). IDMS offers several advantages. It is potentially at least as precise as the GC technique, and is inherently unambiguous since only noble gases survive the chemical purification process. The 40-38-36 isotope system is overdetermined so one can also correct for fractionation effects in the IDMS technique. Although 
the GC technique can be adapted to simultaneously measure $\mathrm{Ar}, \mathrm{O}_{2}$, and $\mathrm{N}_{2}$ on a single sample (Craig \& Hayward, 1987), the IDMS technique can be adapted to measure several noble gases simultaneously (e.g. $\mathrm{He}, \mathrm{Ne}, \mathrm{Ar}, \mathrm{Kr}$; Sano et al., 1982). A quadropole mass spectrometer (QMS) is particularly well-suited for these measurements since it can be tuned to a wide range of masses (e.g. 0-200 amu) and has little "memory" effect due to its small acceleration voltage.

The details of sample collection, processing, and analysis are described below. Samples for argon analysis are collected by gravity-feed from Niskin bottles and stored in copper tubes. In the laboratory, the seawater is quantitatively degassed under vacuum, purified to remove contaminant gases, and the argon is then measured by isotope dilution mass spectrometry. The measurements of argon concentration are compiled in Appendix A.

\subsubsection{Sampling Method}

Samples for argon analysis are collected by gravity-feed from 5-liter Niskin bottles and stored in copper tubes (Figure 2-1; Weiss, 1968; Jenkins \& Clarke, 1976) containing 13 grams of seawater. Several precautions are taken to avoid trapping air bubbles in the sampler and to otherwise insure the integrity of the samples. The tygon tubing is soaked in seawater before using since bubbles tend to form in fresh tubing; this procedure may remove surfactants present in the fresh tubing. Once the Niskin is vented, gravity feed is established slowly to avoid bubble formation. The apparatus is then flushed with several volumes to displace atmospheric gases, trapped bubbles, and water that may have equilibrated with the atmosphere. The sampler is tapped while filling to dislodge any bubbles and drive them out with the flow. After flushing, the $3 / 8$ " OD copper tubing is first clamped at the top ${ }^{1}$ to prevent any loss of water during sealing. Then the bottom clamp is sealed and the extension of the sampler is thoroughly rinsed in fresh water to minimize corrosion.

Although the sampling technique has proven to be quite robust for helium, it has been tested to ensure the integrity of the argon samples. Storage effects were negligible for samples stored up to one year. Samples stored in an argon atmosphere (increasing the potential for contamination one hundredfold) for one year measured within $0.4 \%$ of replicates run immediately after collection.

\footnotetext{
${ }^{1}$ An air-powered socket wrench replaced a hand-operated version after the first six months of sampling
} 

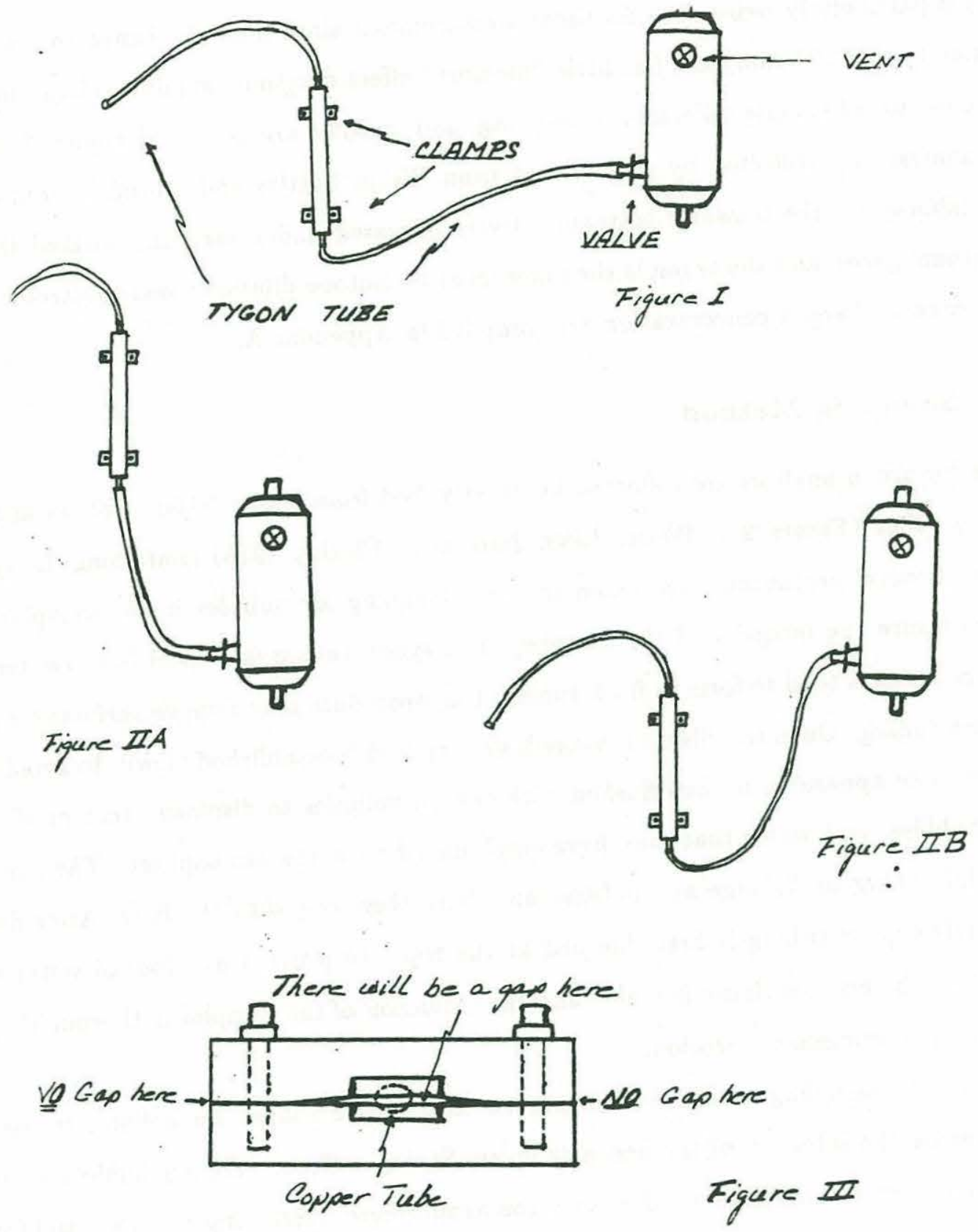

Figure 2-1: Argon sampling procedure from Niskin bottles. 
The most likely source of error is the trapping of air bubbles in the sample tubes. Since the total amount of argon in a $13 \mathrm{~g}$ sample $\left(3 \times 10^{-3} \mathrm{cc} \mathrm{Ar}\right)$ is equivalent to $0.4 \mathrm{cc}$ of air, a bubble of only $0.004 \mathrm{cc}$ ( $1 \mathrm{~mm}$ radius) would create a $1 \%$ supersaturation. Bubbles may form as the seawater warms up "on the wire" or on deck and would then be transferred into the copper tube, where they may adhere to the walls. Bubbles may also form in the tygon tubing, or form in the copper tube itself. These bubbles should be removed from the copper tube sampler by banging it during filling, but it is possible that some may remain.

Another possible mechanism is entrainment of air into the seawater after the Niskin is vented, while the sample is being drawn. This might occur in response to the motion of the ship, which could create turbulence in the Niskin bottle. This effect could not be removed by careful sampling - it is inherent in the process of Niskin sampling itself, unless some other gas were used to backfill the vented Niskin. Shipboard experiments during the SAVE and TPS cruises (pers. comm.) showed that once a 10 liter Niskin bottle is vented, equilibration between dissolved gases (e.g. oxygen) and the headspace can have a significant effect (several percent) within 10-15 minutes. This effect will be minimal for the argon samples, since they are drawn first.

In order to simulate these processes, laboratory experiments were carried out with 10 liter batches of well-mixed artificial seawater $(35 \% \circ \mathrm{NaCl})$. The results (Table 2-1 and Figure 22) suggest that under optimum conditions, the analytical precision of $\pm 0.3 \%$ can be achieved. However, when samples are not taken carefully the precision for replicate samples worsens and larger supersaturations are observed. If gravity feed is established abruptly by quickly lowering the sample tube, turbulent flow may enhance bubble formation. If the sampler is not banged while filling, bubbles may remain in the tube when it is sealed. These effects are exacerbated when the water being sampled is colder than room temperature, possibly because the cold water tends to exsolve bubbles as it warms up during sampling. In addition, if the Niskin bottle is shaken while the sample is drawn the reproducibility also worsens and larger supersaturations are measured. The Niskin was shaken by tilting its vertical axis by approximately $\pm 30^{\circ}$ with a frequency of about $1 \mathrm{sec}^{-1}$; this was intended to exaggerate the rolling of the R/V Weatherbird on a rough day.

In summary, these experiments indicate that under extreme conditions created in the labo- 


\begin{tabular}{lllrrrl}
\hline $\begin{array}{l}\text { Water } \\
\text { temp. }\end{array}$ & & $\begin{array}{l}\text { Sampling } \\
\text { technique }\end{array}$ & $\begin{array}{r}\text { Replicate } \\
\text { precision }\end{array}$ & $\begin{array}{r}\text { Mean } \\
\text { supsat'n }\end{array}$ & $\begin{array}{r}\text { No. of } \\
\text { samples }\end{array}$ & $\begin{array}{l}\text { Histogram } \\
\text { key }\end{array}$ \\
\hline & & & & & & \\
(1) Warm & $\left(22-23^{\circ} \mathrm{C}\right)$ & Good & $\pm 0.3 \%$ & $0.2 \%$ & 3 & horiz. hatch \\
(2) Warm & $\left(22-23^{\circ} \mathrm{C}\right)$ & Poor & $\pm 0.2 \%$ & $0.4 \%$ & 3 & vert. hatch \\
(3) Cold & $\left(4-7^{\circ} \mathrm{C}\right)$ & Good & $\pm 0.3 \%$ & $0.0 \%$ & 9 & unfilled \\
(4) Cold & $\left(4-7^{\circ} \mathrm{C}\right)$ & Poor & $\pm 1.1 \%$ & $0.7 \%$ & 4 & cross-hatch \\
(5) Cold & $\left(4-7^{\circ} \mathrm{C}\right)$ & Good, & $\pm 0.9 \%$ & $1.6 \%$ & 4 & solid \\
& & Sloshing & & & & \\
\hline
\end{tabular}

Table 2-1: Argon sampling experiments using $35 \%$ artificial seawater. Good technique consists of slowly establishing gravity feed in the sample tube and banging the tube while filling it. Poor technique involved lowering the sample tube abruptly and neglecting to bang the tube during filling. "Sloshing" involved shaking the Niskin by tilting its vertical axis by approximately $\pm 30^{\circ}$ with a frequency of about $1 \mathrm{sec}^{-1}$; this was intended to approximate the rolling of the $\mathrm{R} / \mathrm{V}$ Weatherbird on a rough day.

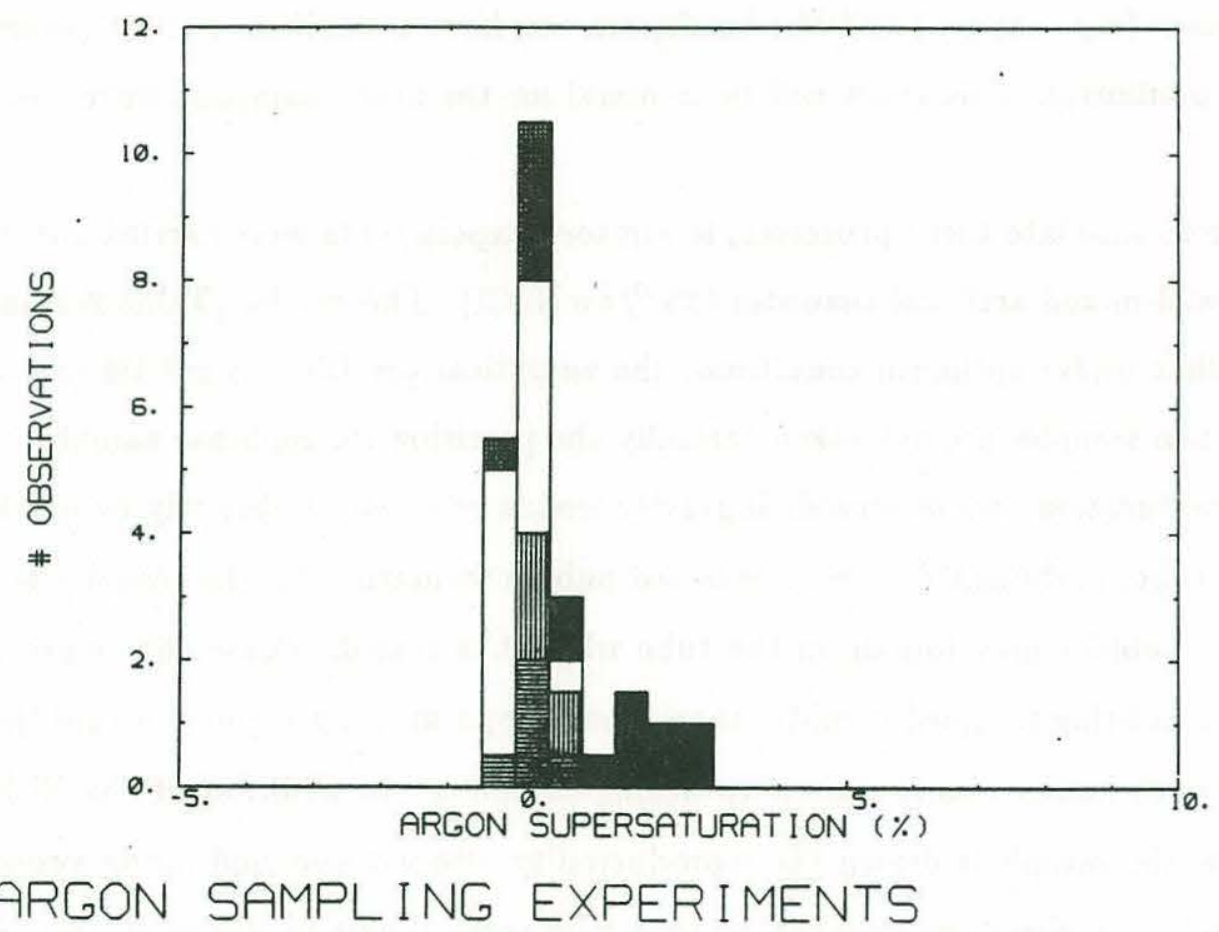

Figure 2-2: Histogram of supersaturations produced in argon sampling experiments. For experiments as listed in Table 2-1: (1) horizontal hatching, (2) vertical hatching,(3) unfilled, (4) cross-hatching, (5) solid. 
ratory measured supersaturations may be inflated by up to $1-2 \%$ as a result of bubble trapping, but more typical conditions yield a $0.0-0.2 \%$ bias. Recent measurements indicate that this problem can be minimized with careful sampling. In total, however, the mean systematic error introduced is small compared to the seasonal range of argon supersaturations (about 3-4\%).

\subsubsection{Argon Analytical System}

The argon extraction, purification, and analysis system is shown schematically in Figure 2-3. The stainless-steel vacuum line is bakeable to $200^{\circ} \mathrm{C}$, and is normally pumped to less than $5 \times 10^{-7}$ torr with a trapped oil diffusion pump (ODP) backed by a 195 liter $\min ^{-1}$ rotary pump (RP). A separate RP in series with a liquid nitrogen cooled water trap (LN-WT) is used to pump the system down to $10^{-3}$ torr and to handle high gas and water vapor loads in the extraction section. Portions of the RP section are operated at $100^{\circ} \mathrm{C}$ to facilitate pumping of water vapor. The titanium sponge getters (TI1 \& TI2) and the $\mathrm{Zr}$-Al getter (SAES) provide additional pumping for the purification section. The standard reservoirs and their associated aliquoting volumes are contained in an aluminum housing to insure thermal equilibrium between the standard reservoirs and aliquoting volumes.

\subsubsection{Extraction}

The water sample is attached to the vacuum system by a Viton 3/8" O-ring compression fitting attached to a bellows. The extraction section can be modified to accept helium-style Pyrex glass breakseal tubes with charcoal; these are used to draw down "library" standards (and blanks) for future comparisons.

Extraction is carried out after evacuating the extraction section to less than $5 \times 10^{-6}$ torr during a 25 minute pumpdown. The section is checked for leaks by measuring the pressure change after 30 seconds isolated from the pumps; a positive pressure surge would be wellcorrelated with an elevated wet line blank and indicate a real or virtual leak. If the pressure surge is negligible, the extraction section is then isolated from the pumps. The bottom seal on the water sample (SC) is rerounded to allow it to drain into a magnetically-stirred $50 \mathrm{cc}$ glass reservoir (DV) attached by a Viton 1/4" O-ring compression fitting ${ }^{2}$. The headspace

\footnotetext{
${ }^{2}$ Originally, a glass-metal seal was used but the O-ring fitting turned out to be sturdier and did not increase
} 


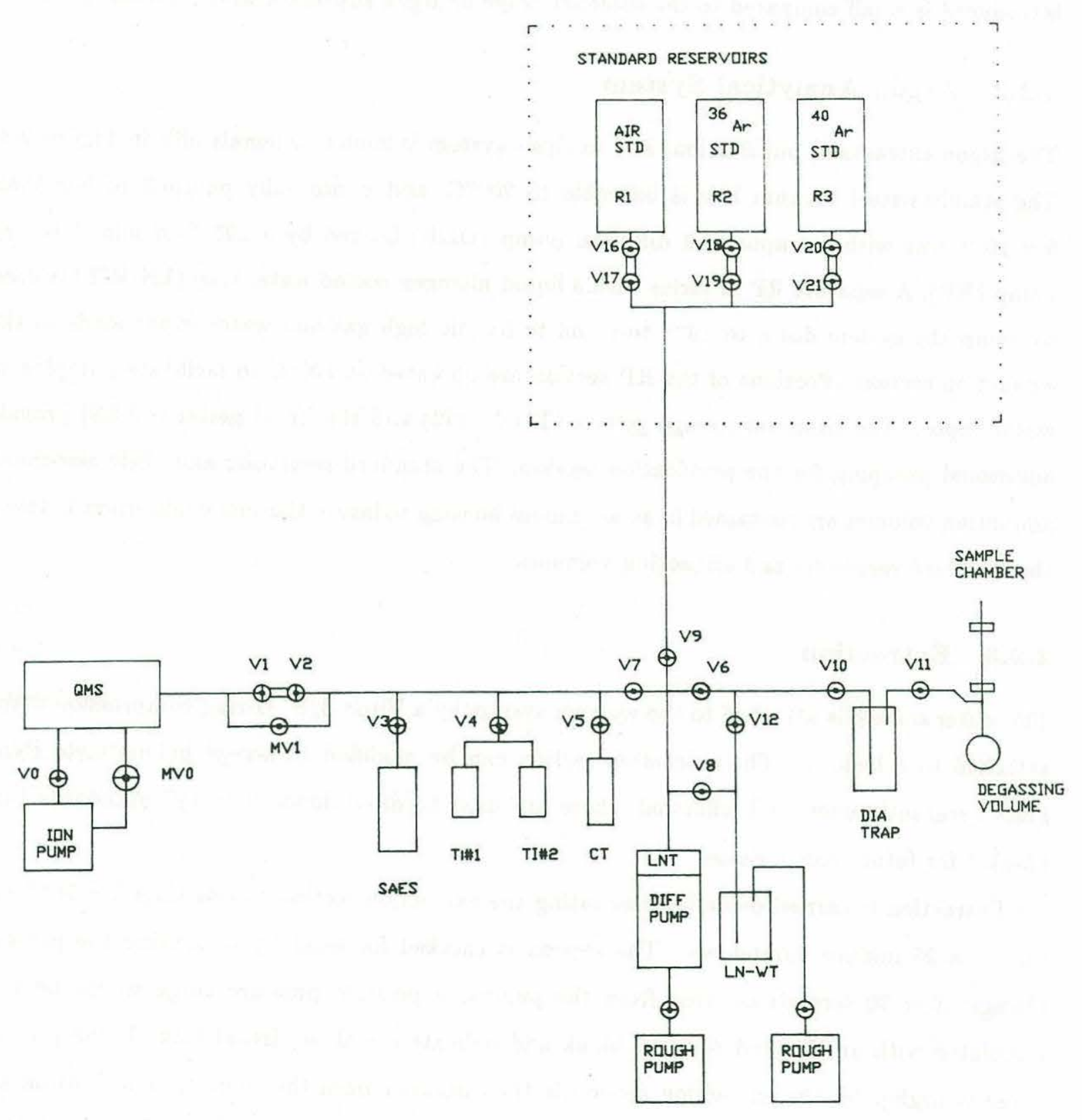

Figure 2-3: Argon extraction, purification \& analysis system. 
gases are vapor pumped through a dry-ice/isopropanol slush trap (DIA) at approximately $-70^{\circ} \mathrm{C}$ to trap water vapor and then drawn onto a charcoal trap (CT) cooled to liquid nitrogen (LN) temperature, $-196^{\circ} \mathrm{C}$. The extraction section is periodically warmed with a gas torch to drive residual water vapor into the liquid reservoir and to encourage equilibration between the reservoir and the entire headspace volume. The seawater is also slightly warmed with the torch to increase the gas diffusivities and decrease the gas solubilities. Since the Bunsen coefficient (gas/water partitioning) for argon is about 30 at room temperature and the headspace/water volume ratio is about 4 , more than $99 \%$ of the argon should be partitioned into the first equilibrated headspace.

About $2 \mathrm{~g}$ of water are transferred to the DIA trap during the extraction. A capillary $(0.040$ " ID $\times 0.75$ " length $)$ is installed between the extraction section and the DIA trap to reduce water vapor transfer and to prevent "backstreaming" of extracted gases.

The results of experiments (Table 2-2) suggest that equilibration between the water sample and the headspace is the rate-limiting step in the extraction, which is consistent with experience in helium extractions. To analyze for "left-behinds" (gases that have not been drawn onto the charcoal trap) in the DIA trap, valve V10 was closed during the second extraction. To analyze for left-behinds in the water, the extraction was then repeated again with valve V10 open. If the measured left-behinds were significantly above blank levels, the extraction was repeated until there was no measurable left-behind. To guarantee quantitative extraction, the degassing is carried out in two cycles (each consisting of a 12 min stir followed by a 3 min draw) with the DIA trap cycled to room temperature in between. This step is crucial since up to $1 \%$ of the argon is left behind in the ice; cycling to room temperature once releases more than $90 \%$ of the trapped argon. Extraction efficiency for water samples is $\geq 99.8 \%$, using the two-cycle method.

The 3 minute drawdown includes a generous safety margin, since $98 \%$ of the argon is drawn onto the CT in 1 minute. However, the CT must be allowed several minutes to cool to LN temperature in between samples. An experiment illustrated that argon could be reversibly adsorbed onto the CT $\left(\tau_{1 / 2}=4 \mathrm{~min}\right.$ after immersing CT in LN) and desorbed $\left(\tau_{1 / 2}<4 \mathrm{~min}\right.$ after removing LN). Once the CT was cold, adsorption was $>99 \%$ complete in 2 min.

the blank. 


\begin{tabular}{lrlll}
\hline Draw/Stir Time & Bulb Size & \multicolumn{3}{c}{ Percent Left-Behind } \\
\cline { 3 - 5 } & & In DIA Trap & In Water & Total \\
\hline 6 min stir \& draw & $50 \mathrm{cc}$ & $0.40 \pm 0.01 \%$ & $0.15 \pm 0.01 \%$ & $0.55 \pm 0.01 \%$ \\
12 min stir \& draw & $50 \mathrm{cc}$ & $1.12 \pm 0.02 \%$ & $0.07 \pm 0.01 \%$ & $1.29 \pm 0.07 \%$ \\
6 min stir \& draw & $200 \mathrm{cc}$ & $0.09 \pm 0.01 \%$ & $0.39 \pm 0.01 \%$ & $0.48 \pm 0.01 \%$ \\
6 min shake \& draw & $200 \mathrm{cc}$ & $0.81 \pm 0.07 \%$ & $0.18 \pm 0.05 \%$ & $0.99 \pm 0.08 \%$ \\
$2 \times(12$ min stir \& & & & & \\
3 min draw) & $50 \mathrm{cc}$ & & & $0.19 \pm 0.06 \%$ \\
\hline
\end{tabular}

Table 2-2: $\quad$ Extraction efficiency experiments.

\subsubsection{Purification \& Diagnostic $\mathrm{He} / \mathrm{Ne}$ Analysis}

A ${ }^{36} \mathrm{Ar}$ spike, pipetted from reservoir $\mathrm{R} 2$, is drawn onto the $\mathrm{CT}$ to ensure complete isotopic mixing. Once the extraction is complete, the CT is isolated. At this point, the extraction section can be cycled for the next sample while the current sample undergoes further processing.

Next, a diagnostic measurement of the helium and neon content of the sample is made ${ }^{3}$. With the CT still at LN temperature, the CT is opened to the purification section (V4, V3) and the gas phase (helium and neon only) is expanded into the QMS by opening V1 and V2. The helium and neon are determined by peak height manometry, using ${ }^{4} \mathrm{He}$ and ${ }^{22} \mathrm{Ne}$ (a high background of ${ }^{40} \mathrm{Ar}^{++}$interferes with measurement of ${ }^{20} \mathrm{Ne}$ ). Reproducibility for air standards is $\pm 6 \%$ over a day; the precision is limited by drift in the QMS sensitivity. For water samples, an additional source of uncertainty is variation in the extraction efficiency. The helium and neon are quantitatively extracted by vapor pumping to the DIA trap; however, they are simply volume partitioned onto the CT since they are not trapped at LN temperatures. Thus, the overall yield is only $43 \pm 2 \%$ overall (a similar fraction of the air standard is partitioned onto the CT), and small variations in the amount left behind can have a significant effect. The reproducibility for helium and neon is estimated at $\pm 7-8 \%$ based on analyses of uncontaminated water samples. The helium background from the ${ }^{36} \mathrm{Ar}$ spike is $50 \pm 3 \%$ of the water sample size; there is no significant neon background ${ }^{4}$. Processing line blanks for helium and neon are $1 \pm 1 \%$, and wet line blanks are $3 \pm 1 \%$ of the sample size.

\footnotetext{
${ }^{3}$ This procedure was implemented while running the second half of the time-series samples.

${ }^{4}$ This background is consistent with diffusion into the glass ampoule during the 1.5 years between the preparation of the standard and its transfer to the steel reservoir.
} 
These diagnostic measurements are used to detect samples that may be contaminated with atmospheric air (due to bubbles trapped in the sampler or leaks). This procedure prevents the getters and QMS from being exposed to large gas loads, and provides a check on sample contamination. As shown in Figure 2-4, samples with high helium and neon values are associated with large argon supersaturations in the proportions expected for atmospheric air contamination. Since helium and neon are 2-4 times more sensitive to air contamination than argon, even the crude precision obtained was adequate to detect contamination for argon of order $1-2 \%$.

Following the helium/neon analysis, the CT is warmed to room temperature to desorb the gases, and then exposed to a hot $\left(800^{\circ} \mathrm{C}\right)$ bulk $\mathrm{Ti}$ sponge getter (TI1); a second $\mathrm{Ti}$ getter (TI2) at room temperature is used to remove hydrogen. The sample is further purified by a $\mathrm{Zr}$-Al SAES getter (at $400^{\circ} \mathrm{C}$ ) to remove any remaining active gases; only noble gases remain. The chemical gettering procedure is quite effective. Most of the active gases (predominantly nitrogen) are gettered by the hot Ti within 1 minute, as measured by the fall in thermocouple pressure (TCG1). Additional gettering by $\mathrm{Zr}-\mathrm{Al}$ is further insurance. Analysis of residual gases in the QMS indicates that there is a negligible increase in "garbage peaks" ${ }^{5}$ on inlet of a sample.

\subsubsection{Analysis}

A precisely reproducible volumetric aliquot (about $0.1 \mathrm{cc}$ corresponding to about $0.05 \%$ ) of the purified noble gas sample is then inlet to the quadropole mass spectrometer (QMS) for analysis. The QMS is a Balzer's QMG 112 with a 0-200 amu mass range (to span the noble gases and to monitor residual gases). The factory-specified sensitivity for ${ }^{40} \mathrm{Ar}$ is $2 \times 10^{-4} \mathrm{~A}$ torr ${ }^{-1}$; a Faraday cup detector provides adequate sensitity for the argon measurements. The mass resolution is 1 amu (10\% valley between peaks) which is adequate to clearly resolve the argon peaks (at 40,38, $36 \mathrm{amu}$ ). The factory-specified stability for peak height and peak height ratio measurements are $\pm 1 \%$ and $\pm 0.5 \%$ respectively over 8 hours. The QMS analyzer is bakeable to $400{ }^{\circ} \mathrm{C}$ for static operation. A 20 liter $\sec ^{-1}$ ion pump is provided for pumping out the QMS and aliquoting volume (V1-V2), with a high conductance Granville-Phillips valve (MV0) for

\footnotetext{
${ }^{5}$ The following masses are measured: $2\left(\mathrm{H}_{2}\right), 4(\mathrm{He}), 14\left(\mathrm{~N}_{2}, 17\left(\mathrm{OH}\right.\right.$ for $\left.\mathrm{H}_{2} \mathrm{O}\right), 28\left(\mathrm{CO}\right.$ and $\left.\mathrm{N}_{2}\right), 32\left(\mathrm{O}_{2}\right)$, and $44\left(\mathrm{CO}_{2}\right)$.
} 
(a)

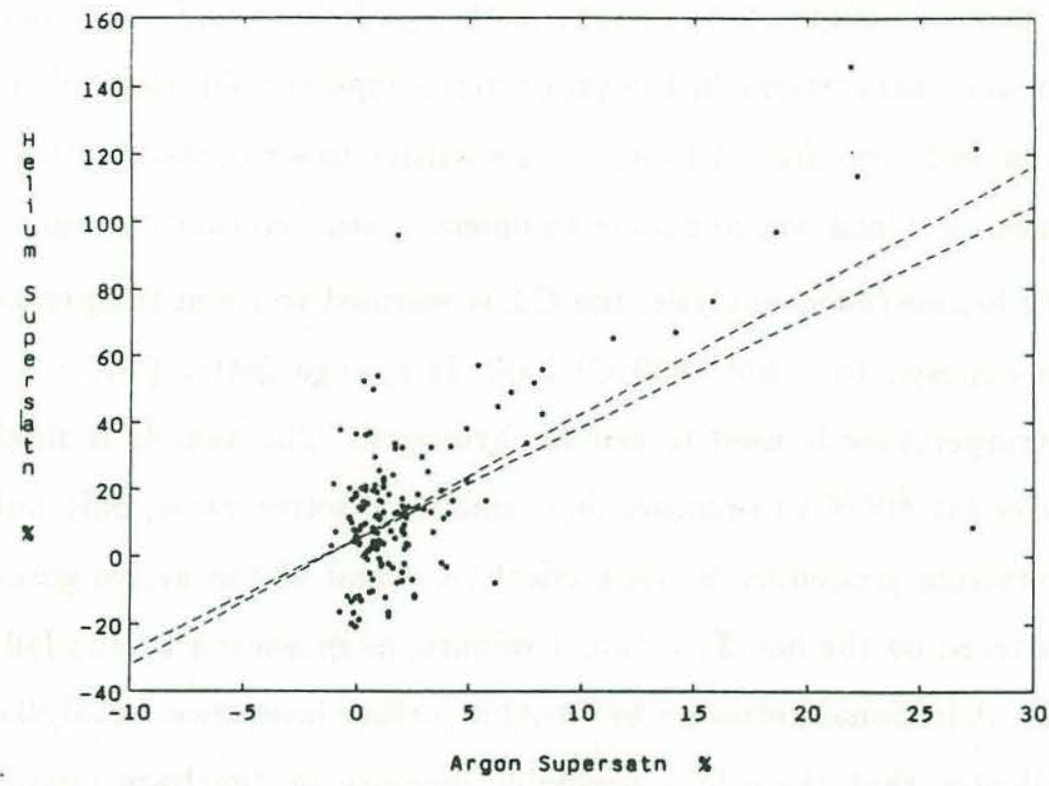

(b)

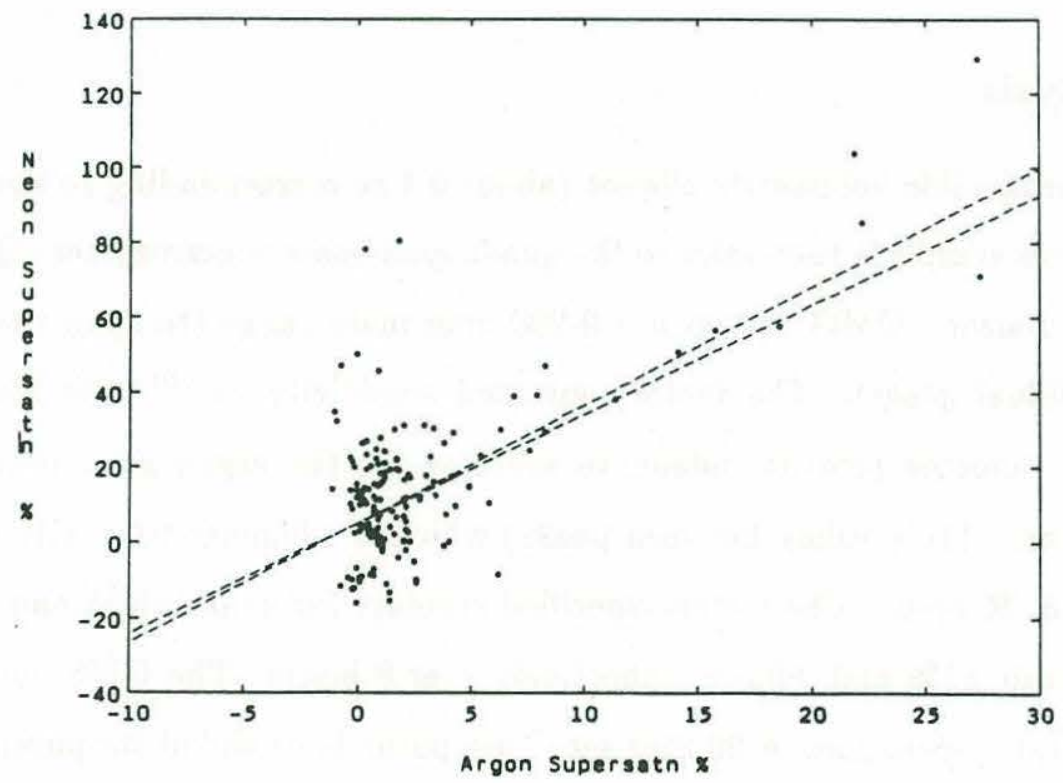

Figure 2-4: Diagnostic measurements of (a) helium and (b) neon. The dashed lines represent the effects of air injection at two different temperatures $\left(19^{\circ} \mathrm{C}\right.$ and $\left.27^{\circ} \mathrm{C}\right)$. 
bakeouts. The QMS pressure is typically less than $7 \times 10^{-9}$ torr, corresponding to a total ion current of $0.1 \times 10^{-11} \mathrm{~A}$ with the QMS in "integral" mode (all ions heavier than 10 amu are detected).

The operating range of the QMS is $5 \times 10^{-7}$ to $5 \times 10^{-6}$ torr; the size of the aliquoting volume (V1-V2) has been and can be adjusted to provide a pressure in this range. Argon is determined both by peak-height manometry of ${ }^{40} \mathrm{Ar}$ and by peak-height ratio using isotope dilution with ${ }^{36} \mathrm{Ar}$. Residual gases (2-50 amu) are also monitored by the QMS and amount to less than $1 \%$ of the sample size. Reservoirs R2 \& R3 with associated aliquoting volumes V18-V19 and V20-V21 are used to deliver ${ }^{36} \mathrm{Ar}$ spikes and ${ }^{40} \mathrm{Ar}$ standards such that two "totes" are within $5-10 \%$ of the water sample size. Reservoir R1 is filled with an atmospheric air standard $\left({ }^{40} \mathrm{Ar} /{ }^{36} \mathrm{Ar}=295.5\right)$ with aliquoting volume V16-V17 calibrated to deliver one tote within 5-10\% of the water sample size $^{6}$. Reservoir volumes are calibrated to better than $\pm 0.1 \%$ by filling with distilled water, and checked against measurements on the standard preparation line described below. Aliquoting volumes are calibrated against glass volumes to $\pm 0.1 \%$ by connecting them in parallel to the air standard and measuring the relative sizes with the QMS.

\subsubsection{Semi-automated Operation}

Automation of the extraction, purification, and analysis is carried out with a Kaypro PC personal computer with an $8088 \mathrm{CPU}$ at $4.77 \mathrm{MHz}$ running MS-DOS version 3.2, an 8087 numeric coprocessor, two $360 \mathrm{~K} 5$ 1/4" floppy disk drives, a 20 megabyte hard disk and a dot-matrix printer. Software is written in Fortran 77 (Lahey version 2.21 compiler), with a few routines written in Microsoft Macro Assembler version 4.00. Hardware specific operations are nested within subroutines to make the software as hardware-independent as possible. Data is transferred to a PDP-11/73 and a DEC MicroVAX for further processing using PRO-MATLAB version 3.33. The PC is interfaced to the processing line through an STD bus with hardware (Metrabyte Corp.) for analog-digital conversion (ADC) to measure QMS output and vacuum pressures, digital-analog conversion (DAC) to control the QMS mass, and parallel TTL I/O ports for valve actuation and getter control. Actuation of the Nupro pneumatic valves is car-

\footnotetext{
${ }^{6}$ Prior to installing the isotopic standards, the large (V16-V17) and small (V18-V19) aliquoting volumes were connected in parallel to the air standard reservoir (R1), and combinations of the two aliquots were used to determine the linearity of the peak height with respect to sample size.
} 
ried out by activating solenoid valves controlled by solid-state relays. The QMS mode and scales are controlled with 15v CMOS logic level-translated from TTL I/O ports.

Although the entire system can be operated in a manual mode, the analysis normally proceeds with limited human intervention. Automation offers a number of advantages. The computer can monitor operating parameters more completely and consistently, detect problems, and take appropriate action (e.g. prevent vacuum accidents) to minimize the effects of operator errors. Automation also leads to more reproducible measurements by providing precise timing and valve actuation and faster and more accurate data acquistion.

The machine cycle time is about 40 minutes. The timing for samples and standards is shown in Tables 2-3 and 2-4. The rate-limiting step is cycling the extraction section. Samples can be run every other machine cycle, so the total time required to run a seawater sample is about 80 minutes. and about 6 water samples can be analyzed in an 8 hour day. Once a water sample has been drawn onto the CT, the extraction section is cycled ("turnaround"). First, the DIA trap is heated with a gas torch and pumped by the LN-trapped RP to remove the bulk of the transferred water. The old sample is then removed, the extraction section is cleaned with distilled water, isopropanol, compressed air, and dry nitrogen and then pumped (by RP and then DP) to $2-5 \times 10^{-6}$ torr. At the end of the day, the RP water trap is warmed and cleaned out with distilled water, isopropanol, and compressed air.

\subsubsection{Standard Preparation}

Isotopic standards are prepared using the spike line diagrammed in Figure 2-5 with NBScertified standards obtained from Monsanto Corporation. Ampoules of $99.99 \pm 0.01 \%$ purity ${ }^{40} \mathrm{Ar}$ and ${ }^{36} \mathrm{Ar}$ isotopic standards (equipped with breakseals) are expanded into the evacuated line using an MKS Baratron capacitance manometer (0-1000 torr $\pm 0.05 \%)$ to measure the pressure; line volumes are calibrated against glass volumes determined to better than $\pm 0.05 \%$ by weighing with water. The MKS unit is factory-calibrated against NBS-certified transfer standards; a series of repeated expansions of dry nitrogen verified that the manometer response is linear within $\pm 0.1 \%$ over the entire pressure range (Figure 2-6). The ballast volumes and the low-conductance RP port are used to adjust the final pressure in the reservoir tank to 1-10 torr. The line is fitted with a charcoal finger which can be cycled to and from LN temperature 


\begin{tabular}{|c|c|c|}
\hline Time (Min) & Standard or Blank & Turnaround \\
\hline-12.0 & Initial stir & \\
\hline 0.0 & Begin processing & Extn section on DP \\
\hline 0.5 & Fetch std aliquots & \\
\hline 3.5 & Begin 15 min wait & \\
\hline 17.0 & Begin draw & \\
\hline 20.0 & Fetch $\mathrm{He} / \mathrm{Ne}$ & \\
\hline 20.5 & Inlet $\mathrm{He} / \mathrm{Ne}$ & \\
\hline 21.0 & Analyze $\mathrm{He} / \mathrm{Ne}$ & \\
\hline 25.0 & Warm CT & Open seal and \\
\hline 25.5 & Begin $\mathrm{Ti}$ & Begin initial stir \\
\hline 28.5 & Begin SAES & Flame extn section \\
\hline 31.5 & Fetch Ar & \\
\hline 32.0 & Inlet to QMS & Flame extn section \\
\hline 32.5 & Warm CT & \\
\hline 35.5 & Pump away & \\
\hline & Print/store data & \\
\hline & Get next sample info & \\
\hline & Wait to cool CT & \\
\hline 40.0 & Begin next analysis & \\
\hline
\end{tabular}

Table 2-3: Argon analysis procedure for standards \& blanks. 


\begin{tabular}{|c|c|c|}
\hline Time (Min) & Water Sample & Turnaround \\
\hline-12.0 & Initial stir & \\
\hline 0.0 & Begin processing & Extn section on DP \\
\hline & Open to DIA $30 \mathrm{sec}$ & \\
\hline 0.5 & Begin draw & \\
\hline 3.5 & End draw & \\
\hline & Begin second stir & \\
\hline & Cycle DIA trap & \\
\hline 7.0 & Replace DIA & \\
\hline 10.5 & Fetch ${ }^{36} \mathrm{Ar}$ spike & \\
\hline 13.5 & Draw ${ }^{36} \mathrm{Ar}$ spike & \\
\hline 16.5 & Open to DIA $30 \mathrm{sec}$ & \\
\hline 17.0 & Begin draw & \\
\hline 20.0 & Fetch $\mathrm{He} / \mathrm{Ne}$ & \\
\hline 20.5 & Inlet $\mathrm{He} / \mathrm{Ne}$ & \\
\hline 21.0 & Analyze $\mathrm{He} / \mathrm{Ne}$ & \\
\hline 25.0 & Warm CT & $30 \mathrm{sec}$ leak test \\
\hline 25.5 & Begin Ti & Open DIA to RP \\
\hline 28.5 & Begin SAES & Backfill w/ $\mathrm{N}_{2}$ \\
\hline 31.5 & Fetch Ar & Clean extn section \\
\hline 32.0 & Inlet to QMS & Open to RP \\
\hline 32.5 & Warm CT & Flame extn section \\
\hline 35.5 & Pump away & Flame extn section \\
\hline & Print/store data & \\
\hline & Get next sample info & \\
\hline & Wait to cool CT & \\
\hline 40.0 & Begin next analysis & \\
\hline
\end{tabular}

Table 2-4: Argon analysis procedure for seawater samples. 
Preparation of Air Standards

Reservoir volume (by water weight) $\quad \pm 0.05 \%$

Atm. pressure (incl. water vapor correction) $\quad \pm 0.03 \%$

Argon atmospheric abundance $\quad \pm 0.11 \%$

Air temperature $\quad \pm 0.02 \%$

Aliquoting volume $\quad \pm 0.05 \%$

Total error $\quad \pm 0.13 \%$

Preparation of Argon Isotope Standards

Spike line manifold volume calibration:

Pressure $\quad \pm 0.05 \%$

Temperature $\quad \pm 0.03 \%$

Glass volume calib. (by water weight) $\quad \pm 0.05 \%$

NBS argon isotope standard size $\quad \pm 0.01 \%$

Line blank correction $\quad<0.01 \%$

Spike line standard expansion:

Pressure $\quad \pm 0.05 \%$

Temperature $\quad \pm 0.03 \%$

Line volumes $\quad \pm 0.08 \%$

Aliquoting volume $\quad \pm 0.05 \%$

Total error $\quad \pm 0.14 \%$

Table 2-5: $\quad$ Errors in standard preparation

to ensure complete mixing in the preparation of isotopic mixtures. This fitting can also be modified to accept a glass breakseal tube with charcoal to measure blanks associated with standard preparation. The spike line is contained in an aluminum housing to buffer short-term fluctuations in room temperature. Several intercalibrated NBS-certified platinum-resistance thermometers (PRT's) are used to monitor thermal gradients in the system to $\pm 0.1^{\circ} \mathrm{C}$. The ideal gas law is used in calculating standard sizes; van der Waals and Virial Theorem corrections amount to less than $0.1 \%$.

For the standard reservoirs, the volumes determined by weighing with water agreed with expansions on the standard preparation line to within $0.2 \%$, although the former were systematically smaller. This was not due to the inclusion of air bubbles when weighing the filled 


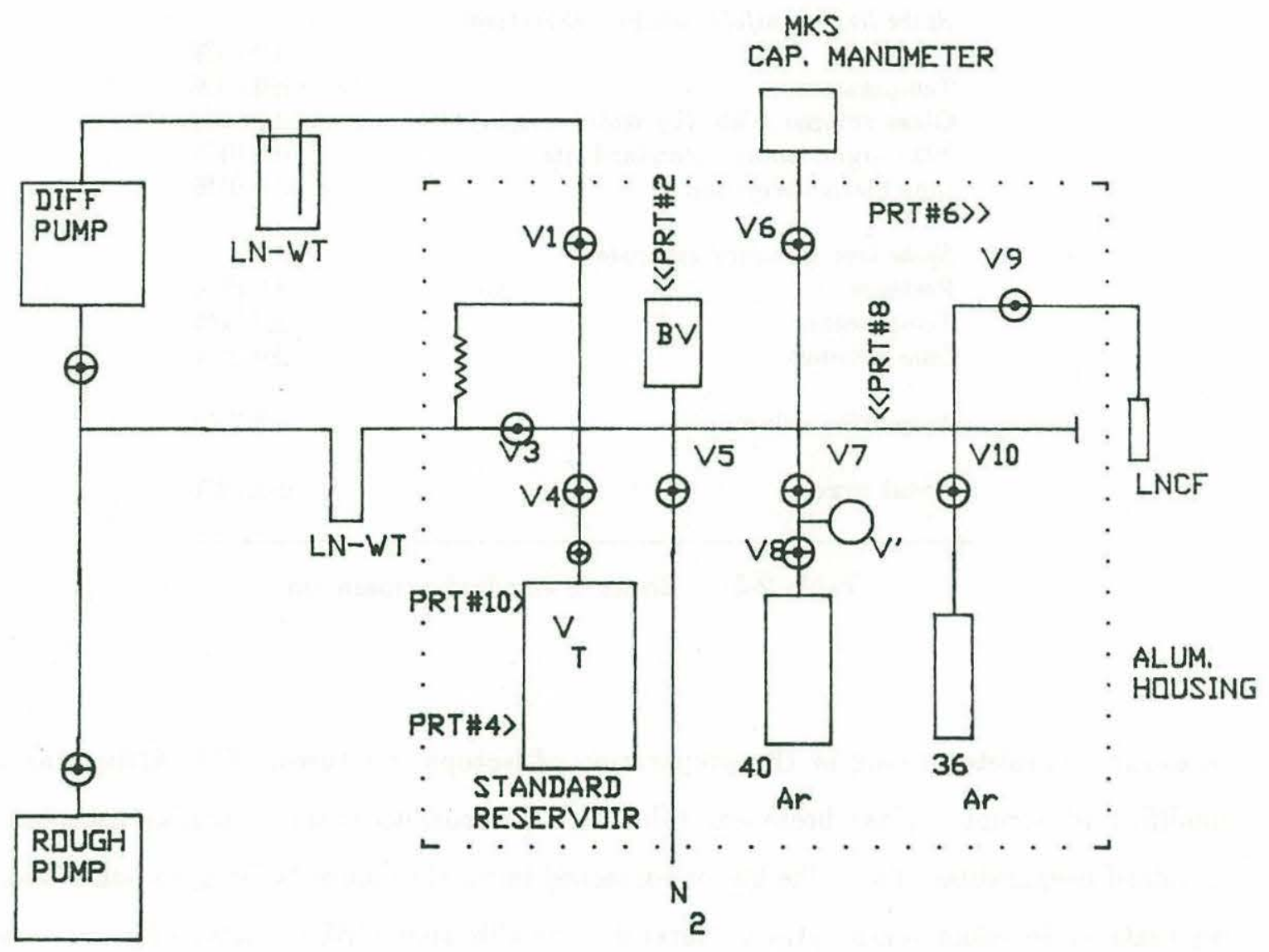

Figure 2-5: Isotope standard preparation line. 


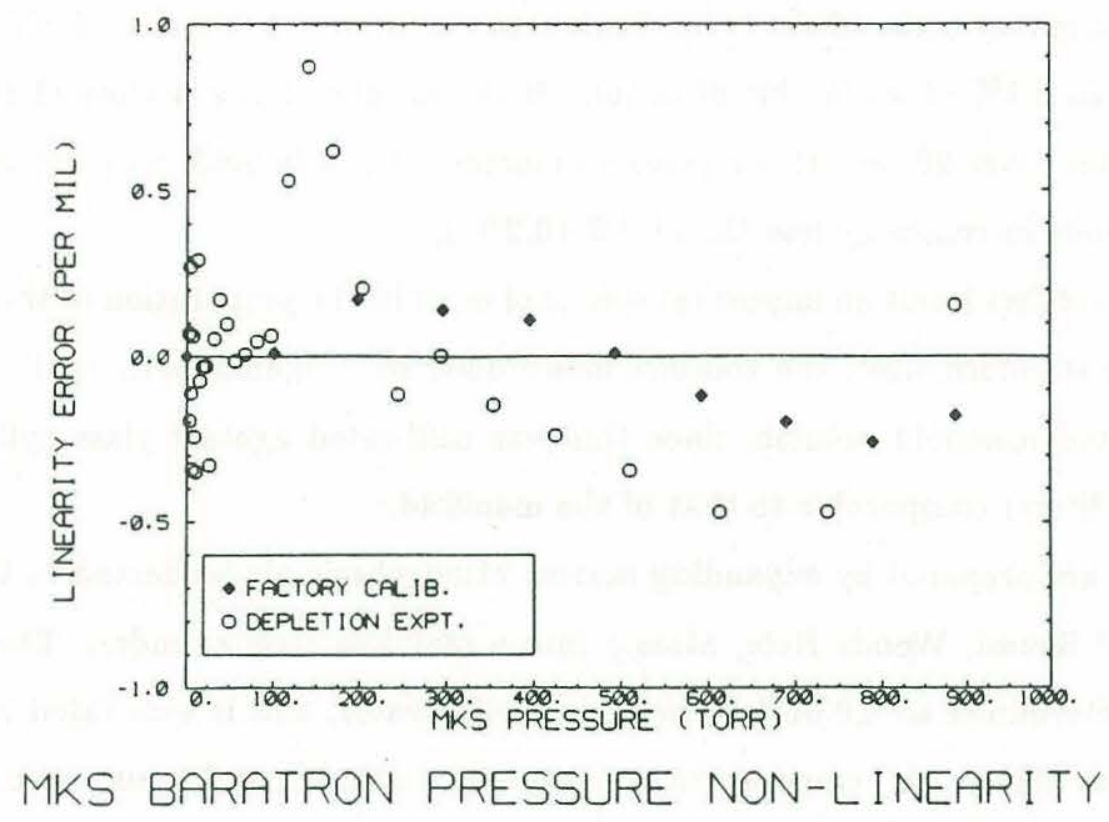

Figure 2-6: Capacitance manometer non-linearity

reservoirs, $20 \mathrm{cc}$ of air would be required to produce this effect, which seems unreasonably large since the weighings were reproducible to within $5 \mathrm{cc}( \pm 0.05 \%)$.

Alternatively, there may be a procedural artifact in the gas expansions. For the preparation of the ${ }^{40} \mathrm{Ar}$ standard, a 1.4 liter volume (containing $1000 \mathrm{cc}$ at STP) was expanded into a $50 \mathrm{cc}$ volume, which was then in turn expanded into the manifold, and subsequently into the 10 liter reservoir. In this case, there was no difference $(<0.01 \%)$ between the volume of the reservoir measured by the spike line and by weighing with water. For the preparation of the ${ }^{36} \mathrm{Ar}$ standard, a 0.2 liter volume (containing $100 \mathrm{cc}$ at STP) was expanded into the manifold, and then in into the 10 liter reservoir. In this case, the volume of the reservoir determined on the spike line was $0.2 \%$ larger than that determined by weighing with water.

When the known manifold volume (1.5 liters) filled with dry nitrogen was expanded into a unknown larger volume (10 liters), the calculated volume of the unknown was $0.2 \%$ (20 cc) larger than if the expansion were done from the large volume to the manifold. For an extreme contrast in volumes ( 0.03 liters vs. 1.5 liters), the discrepancy was almost $3 \%$ ( $0.9 \mathrm{cc})$.

The obvious explanation for this discrepancy is that the gas cools as it performs work in the expansion, resulting in a lower pressure (and hence higher apparent volume). However, 
the cooling associated with an adiabatic or Joule free expansion is less than $0.3^{\circ} \mathrm{C}$ and could account for only an $0.1 \%$ offset in this direction. If the expanded gas is allowed to approach thermal equilibrium (over 20 hours), its pressure increases by only $0.6 \%$ and the temperature of the manifold walls increases by less than $0.1 \%\left(0.2^{\circ} \mathrm{C}\right)$.

However, this artifact is not an important source of error in the preparation of the standards. In calculating the standard sizes, the volumes determined by weighing were used. It does not affect the calculated manifold volume, since this was calibrated against glass cylinders with volumes ( 1 and 2 liters) comparable to that of the manifold.

Air standards are prepared by expanding marine atmospheric air (collected at Chemotaxis Dock on Vineyard Sound, Woods Hole, Mass.) into a stainless steel cylinder. The volume of the reservoir is determined to $\pm 0.05 \%$ by weighing with water, and is evacuated to less than $1 \times 10^{-7}$ torr before filling. Air temperature is measured to $\pm 0.1^{\circ} \mathrm{C}$, and barometric pressure at sea level is measured to \pm 0.01 " and corrected for atmospheric water vapor (relative humidity measured to $\pm 1 \%)$. Air standard size is calculated using the ideal gas law using an atmospheric argon abundance of $0.934 \pm 0.001 \%$ in dry atmospheric air with ${ }^{40} \mathrm{Ar} /{ }^{36} \mathrm{Ar}$ ratio of 295.5 (Weast, 1984).

\subsubsection{Peak Height Manometry}

A tungsten filament has proven to be more reliable than the rhenium filaments initially recommended by the manufacturer. Calculated sensitivity for ${ }^{40} \mathrm{Ar}$ is $0.8 \times 10^{-4} \mathrm{~A} \mathrm{torr}^{-1}$; although this is slightly lower than that specified by the manufacturer, it is more than adequate for this application. Each peak $(40,36,38)$ is scanned in 0.024 amu increments (resolution of the 12-bit DAC controlling the mass); a parabolic fit to the highest 3 points is used to compensate for the limited mass resolution and drift in the QMS mass position. The 0.024 mass resolution was usually able to determine the peak height to within $0.1 \%$ since the peak shapes were relatively smooth (Figure 2-7). In practice, the peak position is stable within $\pm 0.01 \mathrm{amu}$ over several months. The time constant for the QMS response was set to $75 \mathrm{msec}$ to approximate the time required by the computer to acquire the data from the $\mathrm{ADC}$ (which has an integration time of about $50 \mu \mathrm{sec}$. A baseline correction is made by subtracting the ion current measured at 5.0 amu on the same scale (typically a $0.2 \pm 0.01 \%$ offset). 


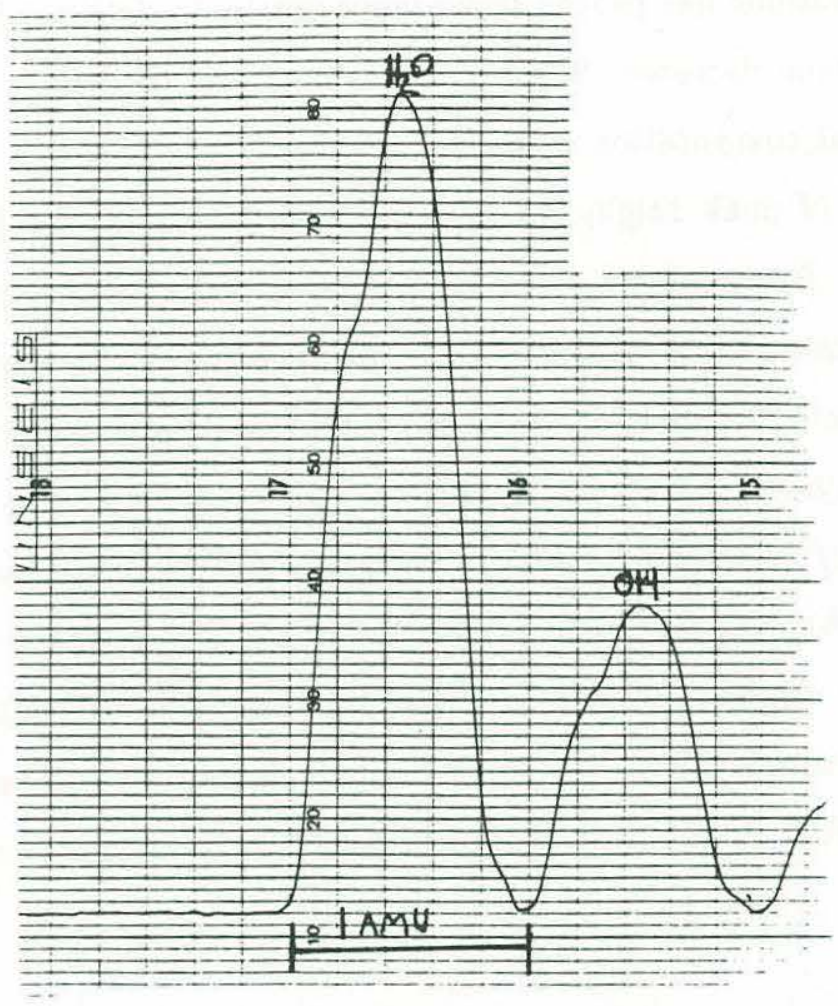

Figure 2-7: Sample QMS spectrum, for $\mathrm{H}_{2} \mathrm{O}(18 \mathrm{amu})$ and $\mathrm{OH}(17 \mathrm{amu})$ on $10^{-9}$ A scale with 75 msec time constant. Note stable baseline, flat peak tops, and clear resolution of the two peaks. The slight asymmetry in shape is a reproducible feature of the QMS ion optics. 
There is a reproducible $5 \%$ decrease in peak heights during the $4 \mathrm{~min}$ analysis, possibly due to consumption of ions by the detector or adsorption of gas on the ceramic insulators. The apparent consumption rate can be reduced by turning off the QMS for part of the analysis, consistent with ion consumption and ruling out a cross-seat leak to the ion pump. An average over the five ${ }^{40} \mathrm{Ar}$ integrations has proven to be more precise in determining peak height than fitting a curve to the mean decrease. Since the data is stored on disk, it is relatively easy to experiment with different computation schemes.

The reproducibility of peak height for air standards is $\pm 0.5 \%$ for ${ }^{40} \mathrm{Ar}$ and $\pm 0.4 \%$ for ${ }^{40} \mathrm{Ar} /{ }^{36} \mathrm{Ar}$ over 8 hours. By running a series of standards and treating alternate standards as "unknowns", it was estimated that unknowns can be standardized by peak height manometry to $\pm 0.1-0.3 \%$. Automated analysis of repeated "totes" of a purified air sample (Figure 2-8) suggest that ${ }^{40} \mathrm{Ar}$ peak height can be determined to $\pm 0.1 \%$ (RMS scatter about the depletion curve), and natural ${ }^{40} \mathrm{Ar} /{ }^{36} \mathrm{Ar}$ ratios to $0.3 \%$. The lower precision for isotopic ratio is due to the smaller size of the ${ }^{36} \mathrm{Ar}$ peak measured on the same scale (atmospheric ${ }^{40} \mathrm{Ar} /{ }^{36} \mathrm{Ar}=295.5$ ); the precision for isotope dilution (with ${ }^{40} \mathrm{Ar} /{ }^{36} \mathrm{Ar} \approx 1$ ) improves to $\pm 0.1 \%$. The QMS peak height response is linear within $2 \%$ over a 2 -fold range in sample size centered on the expected sample size $\left(5 \times 10^{-6}\right.$ torr Ar), with a residual scatter about the linearity curve of $\pm 0.1 \%$ over this range (Figure 2-9).

\subsubsection{Improvements Using Isotope Dilution}

To improve the precision of the QMS analysis, isotope dilution with ${ }^{36} \mathrm{Ar}$ was used. An isotopic "spike" of nearly pure $(99.82 \pm 0.01 \%){ }^{36} \mathrm{Ar}$ was added to the sample on the CT. The size of the spike is usually within $20 \%$ of the amount of ${ }^{40} \mathrm{Ar}$ in the sample. By using isotope dilution, the original isotope ratio of the sample can no longer be determined precisely. However, the total amount of argon in the sample can now be determined much more precisely (assuming we can estimate the original ${ }^{40} \mathrm{Ar} /{ }^{36} \mathrm{Ar}$ of the sample - the ratio for water samples is near that of atmospheric argon). Variations in the partitioning between the QMS aliquot and the purification section and in the sensitivity of the QMS will tend to have the same effect on both isotopes. Any second-order fractionation effects can be corrected for by using ${ }^{38} \mathrm{Ar}$, although the small size of this peak would limit the accuracy of such a correction. For calculating 


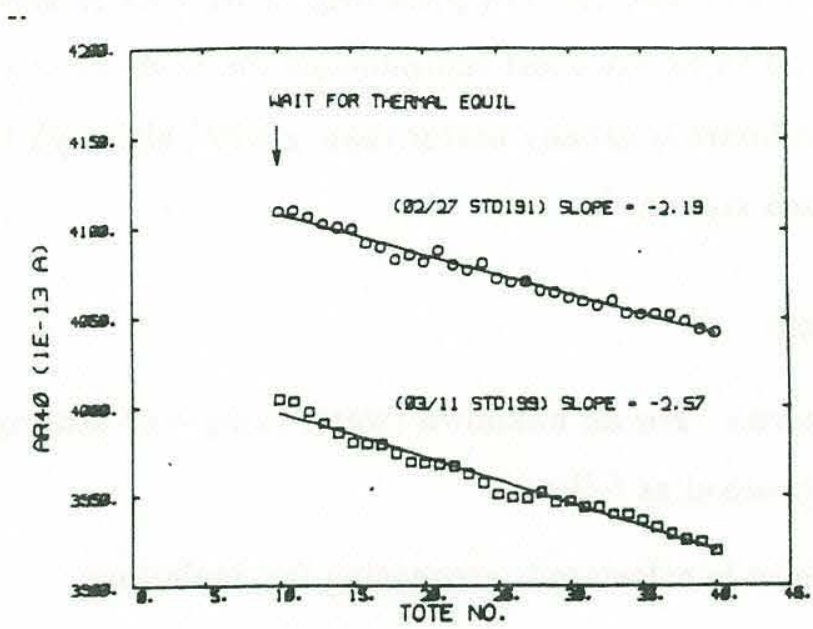

REPT TOTES ( $3 C$ 24C 26C)

Figure 2-8: Automated repeat totes depletion experiment

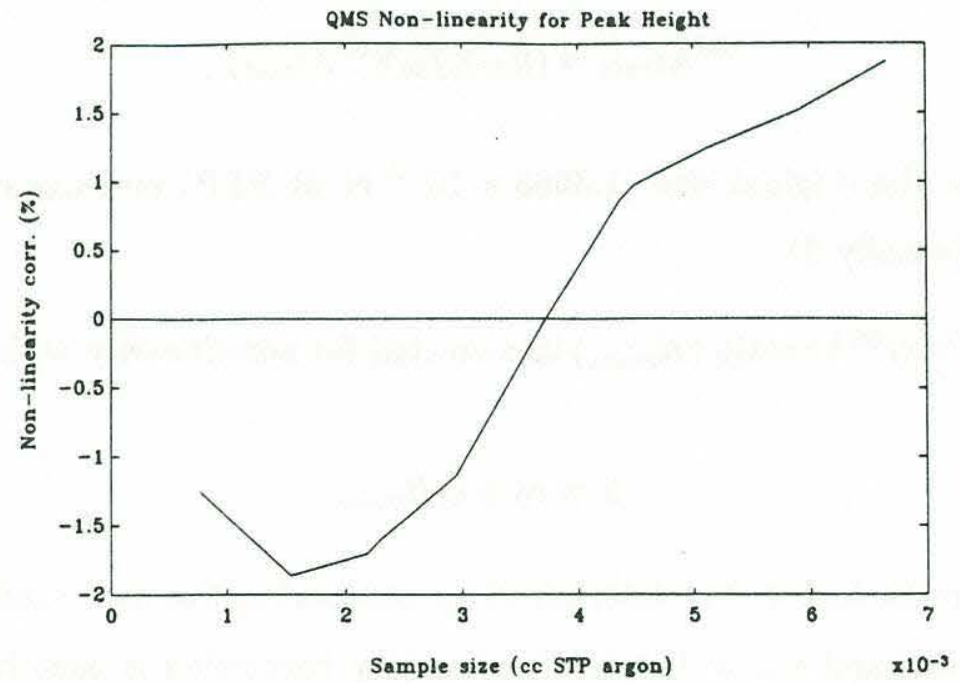

Figure 2-9: Linearity of QMS peak height with sample size 
isotope ratios, the peak heights for each isotope are least-squares fit to a straight line and then extrapolated to the time of inlet. The ratio of peak heights at inlet is used to determine the isotope ratio since this proved to be the most reproducible method. In practice, the precision of the ${ }^{40} \mathrm{Ar} /{ }^{36} \mathrm{Ar}$ ratio over 8 hours is usually better than $\pm 0.2 \%$, although this can be reduced to $\pm 0.1 \%$ by running repeated standards.

\subsubsection{Data Reduction}

Determination of Unknowns For an unknown (water sample or atmospheric air reference standard), the calculations proceed as follows:

1. The size of the ${ }^{36} \mathrm{Ar}$ spike is calculated, accounting for depletion:

$$
f_{36}=\left(1-\varepsilon_{36}\right)^{N_{36}}
$$

where $f_{36}$ is the fraction of the original size remaining, $\varepsilon_{36}$ is the fractional depletion for one "tote" (on the order of $1 \times 10^{-4}$ ), and $N_{36}$ is the total number of totes that have been drawn from the tank. Then,

$$
{ }^{36} \operatorname{Ar}_{c a l c}=\left(n_{36}\right)\left(f_{36}\right)\left({ }^{36} \operatorname{Ar}_{\text {init }}\right)
$$

where ${ }^{36} \mathrm{Ar}_{\text {init }}$ is the original size $\left(1.3663 \times 10^{-3} \mathrm{cc}\right.$ at STP $)$ and $n_{36}$ is the number of totes delivered (usually 2).

2. The measured ${ }^{40} \mathrm{Ar} /{ }^{36} \mathrm{Ar}$ ratio $\left(R_{\text {meas }}\right)$ is corrected for non-linearity with respect to sample size:

$$
\beta=c_{0}+c_{1} R_{\text {meas }}
$$

where the correction factor $\beta$ is determined by comparing the measured ratios for standards with $n_{36}=2$ and $n_{40}=1,2,3$. The linearity correction is usually less than $0.6 \%$ with an uncertainty of $\pm 0.2 \%$ (Figure $2-10$ ).

3. An additional correction $(\alpha)$ is applied for isotopic discrimination:

$$
R_{\text {corr }}=(\alpha)(\beta) R_{\text {meas }}
$$


The value of $\alpha$, determined by running isotopic standards, corresponds to a discrimination against ${ }^{40} \mathrm{Ar}$ of about $4 \%$. This is consistent with discrimination by the QMS source (sensitivity $\propto(\text { mass })^{-1 / 2}$; Dawson, 1976) and diffusive fractionation during inlet to the QMS (diffusivity $\left.\propto(\text { mass })^{-1 / 2}\right)$, which predict a $5 \%$ effect.

4. The size of the unknown $\left({ }^{40} \mathrm{Ar}_{x}\right)$ is computed using the isotope dilution relationship:

$$
{ }^{40} \mathrm{Ar}_{x}=\left({ }^{36} \mathrm{Ar}_{\text {calc }}\right)\left(\frac{R_{\text {corr }}-R_{\text {spike }}}{1-R_{\text {corr }} / R_{x}}\right)
$$

where $R_{x}$ is the assumed isotopic ratio of the unknown $\left(R_{x}=295.5\right.$ for atmospheric air standards and water samples; the isotope effect on solution is negligible). This ratio need not be known precisely as long as it is sufficiently different from the isotope ratio of the ${ }^{36} \mathrm{Ar}$ spike $\left(R_{\text {spike }}=8.0 \times 10^{-4}\right)$.

5. For an atmospheric air reference standard, the measured size is compared with the calculated size as a check on the integrity of the isotopic standards (see Figure 2-12).

6. For a water sample, the total amount of argon (all isotopes) in the sample is computed by accounting for the natural isotopic abundances $(\mathcal{I}=1.0040)$, a buoyancy correction to the water weight $(\mathcal{B}=0.9985)$, the wet line blank $(\mathcal{W})$, and the extraction efficiency $(>99.8 \% ; \mathcal{E}=1.00190)$ :

$$
\operatorname{Ar}_{x}=\left({ }^{40} \operatorname{Ar}_{x}-\mathcal{W}\right)(\mathcal{E})(\mathcal{B})(\mathcal{I})
$$

Typical wet line blanks, obtained by processing samples without opening the seal, are $1.06 \pm 0.60 \times 10^{-5} \mathrm{cc}{ }^{40} \mathrm{Ar}$. However, the first sample run each day has a much lower blank $\left(0.06 \pm 0.21 \times 10^{-5} \mathrm{cc}{ }^{40} \mathrm{Ar}\right)$ since it has been pumped overnight. Finally, the weight of the water sample is used to measured compute the argon concentration:

$$
\mathrm{C}_{\text {Ar }}=\mathrm{Ar}_{x} /\left(W t_{\text {full }}-W t_{\text {empty }}\right)
$$

Concentrations are measured as (cc at S.T.P.) (g seawater) ${ }^{-1}$. Saturation anomalies $(\Delta \mathrm{Ar})$ are expressed as percent deviations from smoothed gas solubilities compiled by Weiss $(1970 ; 1971)$, which have a precision of about $0.2 \%$. Benson and Krause (1976) 


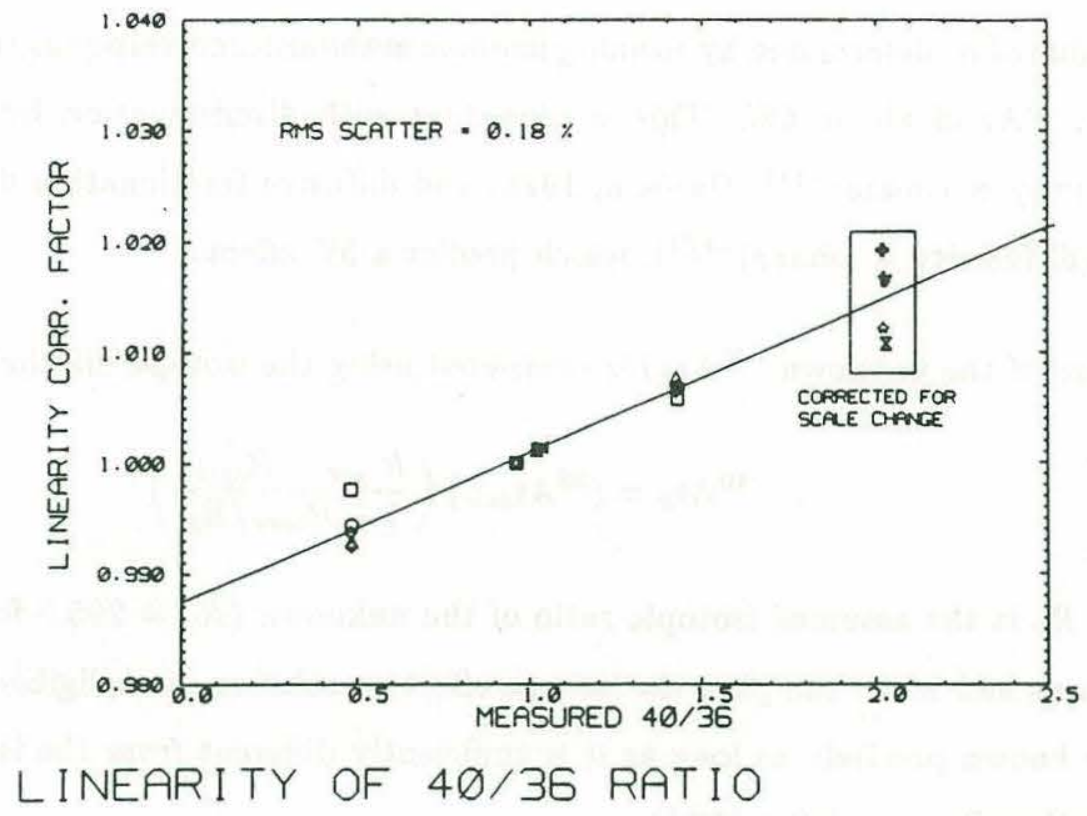

Figure 2-10: Non-linearity of QMS peak height ratio with sample size

report solubilities about $0.2 \pm 0.1 \%$ lower than those of Weiss $\left(0.3 \%\right.$ at $0^{\circ} \mathrm{C}$ to $0.1 \%$ at $30^{\circ} \mathrm{C}$ ). A systematic uncertainty of this order is possible, but is comparable to the accuracy of the concentration measurements.

Standard Determinations For a running standard (2 totes each of ${ }^{40} \mathrm{Ar}$ and ${ }^{36} \mathrm{Ar}$ ), the calculations are as follows:

- The amounts of each isotope $(40,36)$ delivered are calculated, accounting for depletion:

$$
f_{i}=\left(1-\varepsilon_{i}\right)^{N_{i}}
$$

where $f_{i}$ is the fraction of the original size remaining, $\varepsilon_{i}$ is the fractional depletion for one "tote" (on the order of $1 \times 10^{-4}$ ), and $N_{i}$ is the number of totes that have been drawn from the tank. As above,

$$
{ }^{36} \mathrm{Ar}_{\text {calc }}=\left(n_{36}\right)\left(f_{36}\right)\left({ }^{36} \mathrm{Ar}_{\text {init }}\right)
$$


where ${ }^{36} \mathrm{Ar}_{\text {init }}$ is the original size $\left(1.3663 \times 10^{-3}\right.$ cc at STP $)$ and $n_{36}$ is the number of totes delivered (2). Similarly,

$$
{ }^{40} \mathrm{Ar}_{\text {calc }}=\left(n_{40}\right)\left(f_{40}\right)\left({ }^{40} \mathrm{Ar}_{\text {init }}\right)
$$

where ${ }^{40} \mathrm{Ar}_{\text {init }}$ is the original size $\left(1.3609 \times 10^{-3} \mathrm{cc}\right.$ at STP $)$ and $n_{40}$ is the number of totes delivered (2).

- The expected ratio is compared with the measured ratio $\left(R_{\text {meas }}\right)$ to calculate a new value for $\alpha$, the isotopic discrimination factor:

$$
\begin{gathered}
\xi={ }^{40} \operatorname{Ar}_{\text {calc }} /{ }^{36} \operatorname{Ar}_{\text {calc }} \\
\alpha_{\text {calc }}=\frac{\xi+R_{\text {spike }}}{R_{\text {meas }}\left(1-\xi / R_{\text {std }}\right)}
\end{gathered}
$$

where $R_{\text {std }}=5003.7$ for a running standard.

Processing line blanks are measured by running a "standard" with no aliquots drawn from the tanks; typical values are $0.2 \pm 0.1 \%$ of the standard size. Virtually all of the line blank is contributed by "memory" in the QMS. Since the blank is nearly the same for all samples and standards, the correction is negligible $(<0.1 \%)$.

\subsubsection{Peak Height Manometry Results}

Preliminary results using peak height manometry indicate that precision for replicates drawn from Vineyard Sound surface water is $\pm 0.6 \%$ (Table 2-6). The mean measured $C_{A r}=3.033 \pm$ $0.018 \times 10^{-4} \mathrm{cc} \mathrm{g}^{-1}$, compared to the solubility $C_{A r}^{*}=3.06 \pm 0.04 \times 10^{-4} \mathrm{cc} \mathrm{g}^{-1}\left(T=10.2^{\circ} \mathrm{C}\right.$, $S=32 \pm 1 \% \circ$ ). Thus, the measured value is within $1 \%$ of the normal atmospheric equilibrium concentration. Some of the scatter may be due to weighing errors of up to $\pm 0.25 \%$ in 13 g samples $( \pm 0.03 \mathrm{~g})$. The balance has subsequently been replaced with a unit accurate to $\pm 0.08 \%$ (Mettler PE1600, accurate to $\pm 0.01 \mathrm{~g}$ ).

Without isotope dilution, ${ }^{40} \mathrm{Ar} /{ }^{36} \mathrm{Ar}$ ratio for air standards and water samples is $278.0 \pm 1.0$, about $6 \%$ less than the atmospheric ratio (295.5). Most of this variation can be attributed to mass discrimination by the QMS or fractionation during inlet to the QMS, which are predicted 
to vary with (mass) $)^{-1 / 2}$ (Dawson, 1976) and would account for a $5 \%$ effect. The isotope ratios for air standards and water samples are indistinguishable, setting an upper limit of $0.3 \%$ to the equilibirum isotope effect on solution.

\subsubsection{Isotope Dilution Results}

Replicate analyses of Vineyard Sound surface water obtained by bucket sampling and measured by isotope dilution indicate that the total uncertainty associated with copper tube sampling, storage, extraction and mass spectrometric analysis is $0.3 \%$ (see Table 2-6). Absolute calibrations of the isotopic standards, volumes and temperatures are accurate to $0.15 \%$ and traceable to NBS. The long term mean value of the isotope discrimination factor $\alpha$ is $1.0377 \pm 0.0022$ determined by running isotopic standards over more than one year (Figure 2-11). A systematic downward trend in $\alpha$ amounts to a $0.3 \%$ decrease over the duration of the analyses, associated with a $3 \%$ depletion of the ${ }^{40} \mathrm{Ar}$ and ${ }^{36} \mathrm{Ar}$ isotopic standards during this period. Sample sizes are corrected by determining a daily mean value for $\alpha$ (typically to within \pm 0.001 ). Air standards run over the 1.5 years of seawater analyses indicate that unknown sample sizes can be determined to within $\pm 0.2 \%$ with no long term, systematic trends (Figure 2-12). A series of 20 samples from a depth of $2000 \pm 200$ meters show no significant trends with a mean $\Delta \mathrm{Ar}$ of $-0.7 \%$, but have a somewhat larger sample standard deviation of $0.6 \%$ (Figure 2-13) ${ }^{7}$. This is an upper bound to the analytical error because it includes any natural variations (presumed to be small at this depth), but may indicate a contribution from sampling artifacts as discussed above.

\subsubsection{Sampling Errors}

The reproducibility of samples from Bermuda is somewhat worse than the analytical precison (Table 2-6 and Figure 2-15). The precision of replicates drawn from the same Niskin bottle is about $1 \%$ on average (range $0.1-4.0 \%$ ); this may be due to bubble trapping as discussed below. The precision of mixed layer average concentrations is also about $1 \%$ on average (range $0.1-2.0 \%$ ), although for winter profiles it is typically better than $0.3 \%$; this is an upper limit to

\footnotetext{
${ }^{7}$ These statistics were calculated after two samples with supersaturations greater than 3 standard deviations from the mean were removed.
} 


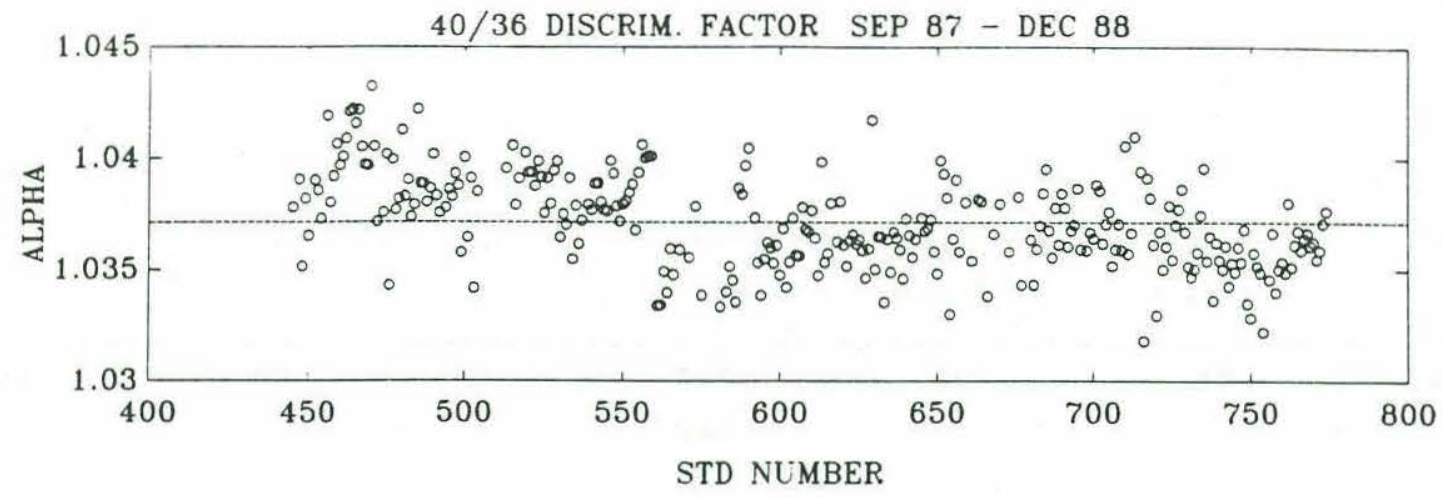

Figure 2-11: Isotopic discrimation factor $(\alpha)$ vs. standard number for more than one year of analyses.

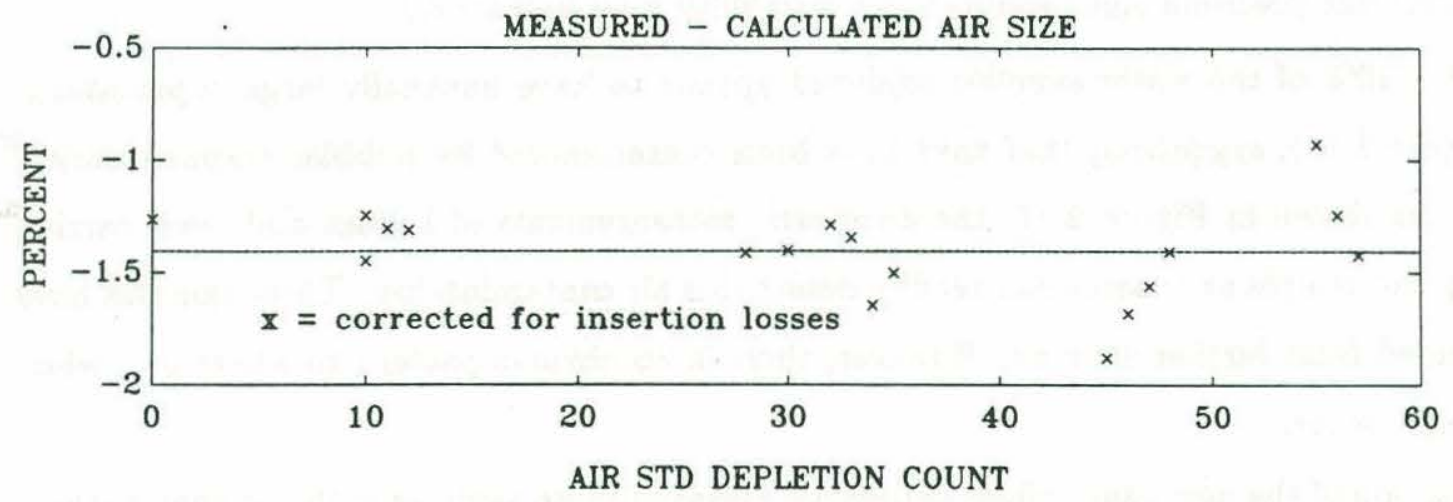

Figure 2-12: The measured size of the air standard compared to the calculated size vs. time. The sizes are corrected for "insertion losses" incurred when removing and reinstalling the air standard tank several times during the 1.5 years of analyses. 


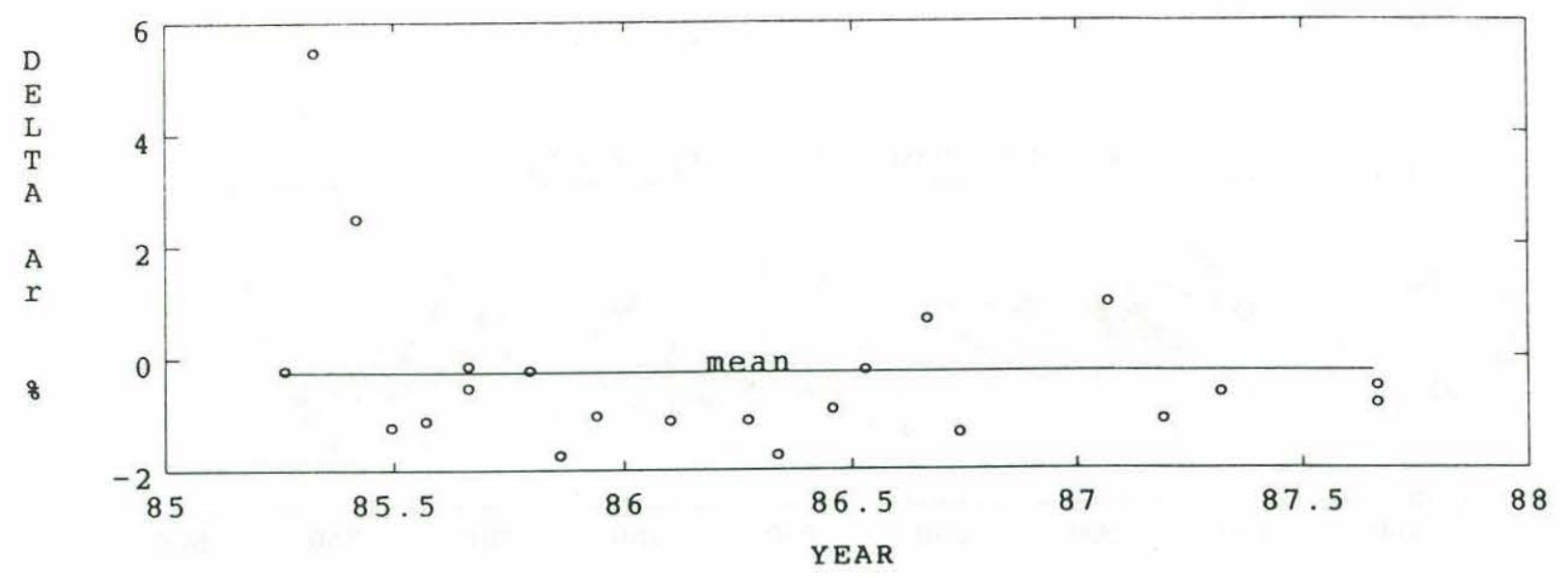

Figure 2-13: Measured argon supersaturations in deep (2000 m) water.

the measurement precision since some of the variability may be natural.

About $5-10 \%$ of the water samples analyzed appear to have unusually large supersaturations (Figure 2-14), suggesting that they have been contaminated by bubbles trapped during sampling, As shown in Figure 2-16, the diagnostic measurements of helium and neon carried out during the sample processing can readily detect this air contamination. These samples have been excluded from further analysis. However, there is no obvious pattern to where and when these "fliers" occur.

Comparison of the apparent helium supersaturations of these samples with the supersaturations of independent $45 \mathrm{~g}$ helium samples supports the interpretation that the air is an artifact of copper tube sampling rather than a problem with the Niskin bottle itself (or a result of air injection in the ocean), since the anomalies in the two sets of samples are not well-correlated. Examination of the helium concentration data set suggests that a similar proportion (3-5\%) of the helium samples are also compromised by bubble trapping (or leaks during sample storage or extraction). Argon may be more sensitive to this source of contamination because of the stronger temperature dependence of its solubility. 


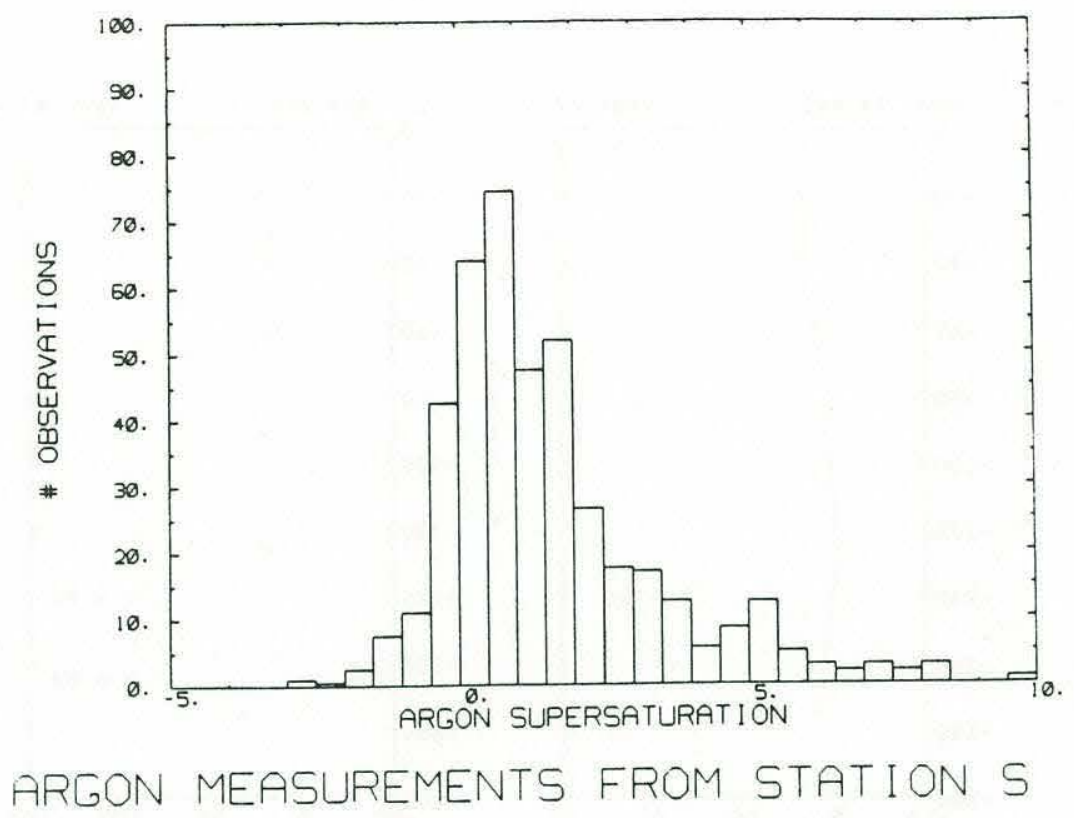

Figure 2-14: Histogram of argon supersaturations measured at Station S.

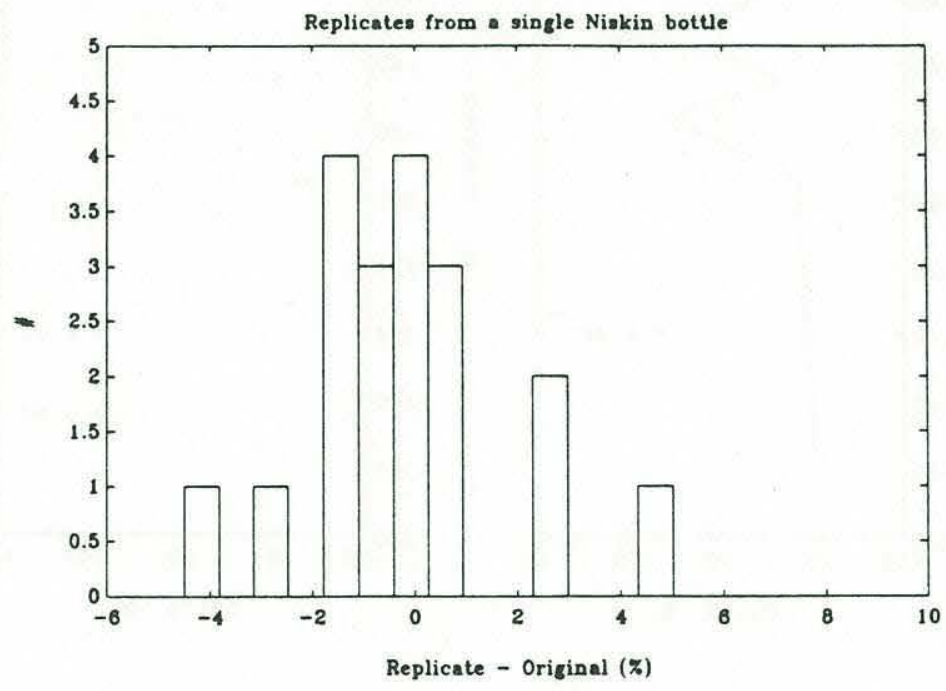

Figure 2-15: Histogram of differences between replicate samples drawn sequentially from a single Niskin bottle. 

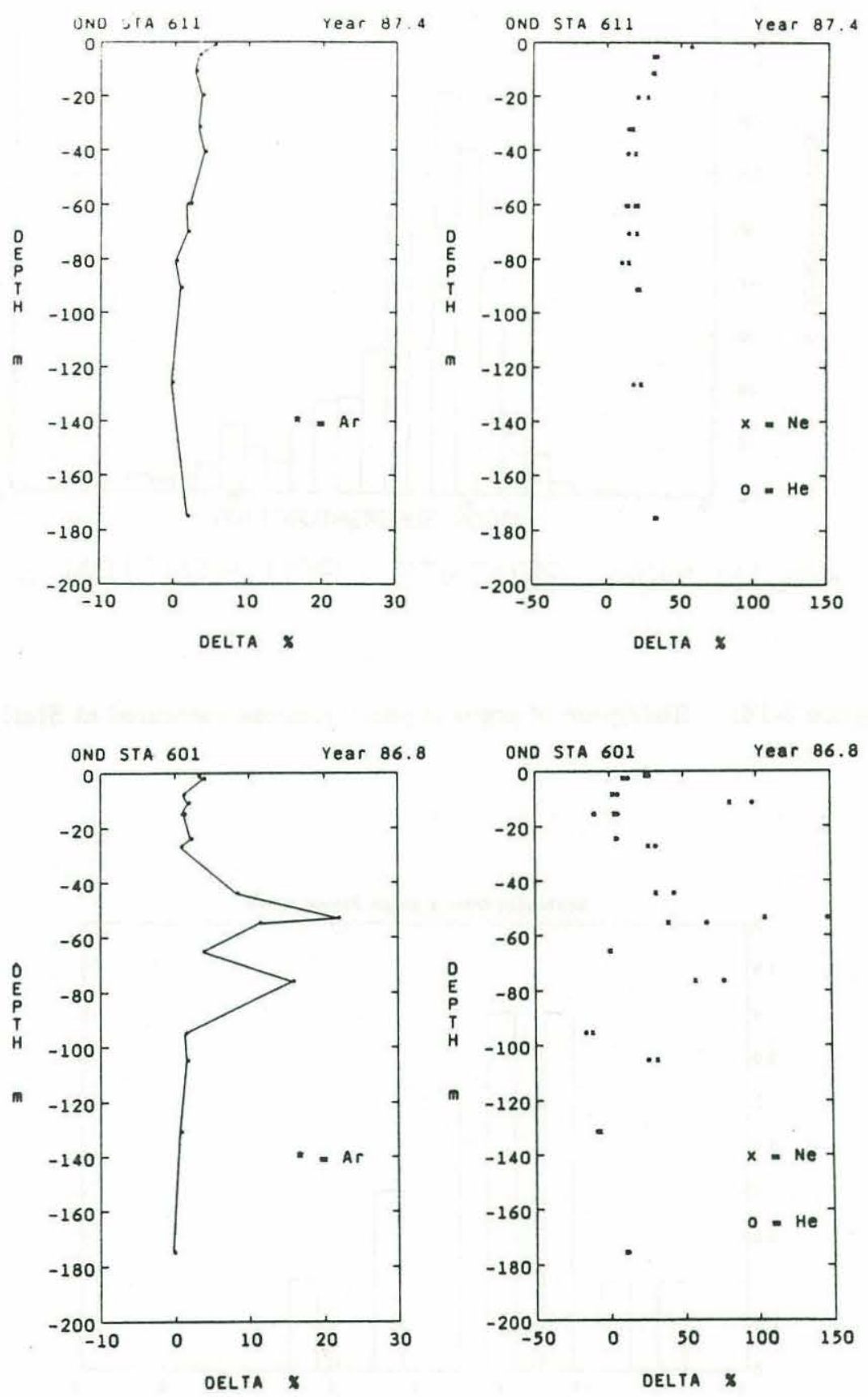

Figure 2-16: Use of diagnostic helium and neon measurements to detect air contamination for two profiles. A late winter profile (upper panels) with relatively small and constant argon supersaturations is characterized by nearly constant values of helium and neon. A summer profile (lower panels) contains several samples with high argon supersaturations. These samples also have high values of helium and neon in the ratios expected for air contamination. Independent helium samples do not show these anomalies, supporting this interpretation. 
Analytical ERrors

Sampling

$<0.5 \%$

Extraction efficiency

$99.8 \pm 0.1 \%$

Blank correction

$0.3 \pm 0.2 \%$

$\pm 0.1 \%$

QMS Analysis (Isotope dilution with $40 / 36 \approx 1.0 \pm 0.2$ )

$0.6 \pm 0.2 \%$

QMS linearity correction

Weighing

$\pm 0.1 \%$

Total analytical error

$\pm 0.3 \%$

Systematic Errors

Sampling, storage

$<0.3 \%$

Standard preparation

$\pm 0.15 \%$

Argon solubility (for calculating $\Delta \mathrm{Ar}$ )

$( \pm 0.2 \%)$

Total systematic error

$\pm 0.2 \%$

\section{Actual Reproducibility}

10 Vineyard Sd. bucket samples (by peak height manometry) $\quad \pm 0.6 \%$

10 Vineyard Sd. bucket samples (by isotope dilution) $\quad \pm 0.3 \%$

20 deep $(2000 \mathrm{~m})$ samples (mean $\Delta \mathrm{Ar}=-0.7 \%) \quad \pm 0.6 \%$

Average precision of replicates drawn from same Niskin bottle $\quad \pm 1.0 \%$

Average precision of mixed layer averages $\quad \pm 1.0 \%$

Table 2-6: Random and systematic errors in argon measurements. 


\begin{tabular}{ll}
\hline Measurement & Precision \\
\hline${ }^{3} \mathrm{H}$ (stored 1 yr) & $\pm 0.5 \%$ or $\pm 0.04 \mathrm{TU}$ \\
${ }^{3} \mathrm{He} /{ }^{4} \mathrm{He}$ & $\pm 0.18 \%$ \\
$\mathrm{C}_{\mathrm{He}}$ & $\pm 0.2 \%$ \\
${ }^{3} \mathrm{H}-{ }^{3} \mathrm{He}$ age & $\pm 0.18 \mathrm{yr}$ \\
$\quad$ (surface ocean 3.5 TU) & \\
\hline
\end{tabular}

Table 2-7: Precision of tritium and helium measurements on $45 \mathrm{~g}$ samples.

\subsection{Tritium, Helium and Neon Analyses}

Helium samples are collected in copper tube samplers containing 45 grams of seawater, and the total helium content is measured by peak height manometry to a precision of $0.2 \%$ (Lott and Jenkins, 1984). ${ }^{3} \mathrm{H}-{ }^{3} \mathrm{He}$ are also measured by mass spectrometry (Jenkins, 1987), with precision as indicated in Table 2-7. Concentrations are measured as (cc at S.T.P.) (g seawater) ${ }^{-1}$. Saturation anomalies $(\Delta \mathrm{He})$ are expressed as percent deviations from smoothed gas solubilities compiled by Weiss (1971b), which have a precision of $0.3 \%$ and possible systematic uncertainty of $0.5 \%$.

\subsection{Hydrographic Data}

Since the seasonal cycle of oxygen will be used to infer rates of new production, the analytical error in the oxygen measurements must also be assessed. Oxygen is measured at the Bermuda Biological Station (BBS) by a modified Winkler titration (Carritt \& Carpenter, 1966). Carritt \& Carpenter (1966) suggest that "a good analyst" can make determinations to at least \pm 0.05 $\mathrm{ml} / \mathrm{l}$ and the experience of the PACODF Group suggests that it is possible to do as well as $\pm 0.01 \mathrm{ml} / 1$ with careful attention to sampling and determinations of blanks and standards.

Using the data from 1954-1988, temperature, salinity, and oxygen were linearly interpolated to $\sigma_{\theta}=27.85 \%$ with a window of $\pm 0.1 \%$. The results are shown below in Table $2-8$. Similar results are obtained by polynomial fits of these properties as a function of density over the range $\sigma_{\theta}=26.2-27.9$ (Table 2-9 and Figure 2-17), although the natural variability appears somewhat larger over this domain. However, the spread of the residuals for each station tends to minimize the effects of the long-term variations, and is probably closer to the analytical 


\begin{tabular}{lrr}
\hline Property & $\bar{x}$ & $\sigma_{s}$ \\
\hline Depth $(\mathrm{m})$ & 2024 & 107 \\
Temperature $\left({ }^{\circ} \mathrm{C}\right)$ & 3.73 & 0.08 \\
Salinity $(\% \circ)$ & 34.99 & 0.02 \\
Oxygen $\left(\mathrm{ml} \mathrm{l}^{-1}\right)$ & 6.05 & 0.14 \\
\hline
\end{tabular}

Table 2-8: Station S data for 1954-1988 interpolated to $\sigma_{\theta}=27.85$. The means and sample standard deviations were recomputed after deleting data (typically about $1 \%$ ) that deviated from the mean by more than 3 standard deviations. Oxygen measurements prior to 1961 were not included since they suffered from standardization problems (Jenkins, 1982).

\begin{tabular}{lccc}
\hline Property & $\mathrm{N}$ & \% removed & $\sigma_{s}$ \\
\hline Salinity $(\% \circ)$ & 9466 & 1.0 & 0.05 \\
Oxygen $\left(\mathrm{ml} \mathrm{l}^{-1}\right)$ & 7043 & 1.8 & 0.23 \\
\hline
\end{tabular}

Table 2-9: Fourth-order least-squares polynomial fits of salinity and oxygen to density over the range $\sigma_{\theta}=26.2-27.9$. The curves were recomputed after deleting data that deviated from the mean by more than 3 standard deviations. Oxygen measurements prior to 1961 were not included since they suffered from standardization problems (Jenkins, 1982).

precision.

J\&G estimated an upper bound to the analytic error in the BBS oxygen analyses from the reproducibility of deep water concentrations; during 1961-1978 the sample standard deviation at 2000 meters was $0.125 \mathrm{ml}^{-1}$, with no significant seasonal or long-term trends. During 1985-1987, the reproducibility at this depth is comparable $\left( \pm 0.15 \mathrm{ml} \mathrm{l}^{-1}\right)$ and similar estimates were obtained by comparing duplicate analyses $\left( \pm 0.17 \mathrm{ml} \mathrm{l}^{-1}\right)$ and by computing the scatter about a mean climatological oxygen vs. temperature curve $\left( \pm 0.19 \mathrm{ml} \mathrm{l}^{-1}\right.$ for $\left.3^{\circ} \mathrm{C}<\mathrm{T}<16^{\circ} \mathrm{C}\right)$ for the 1985-1987 measurements. During 1985-1987, the mean value at 2000 meters was 6.12 $\mathrm{ml}^{-1}$, just slightly higher (by $0.06 \mathrm{ml} \mathrm{1}^{-1}$ ) than the $1961-1978$ mean $^{8}$.

Nutrients (nitrate + nitrite and phosphate) are also measured at BBS using the standard Cd-column reduction and spectrophotometric technique (Strickland \& Parsons, 1967). Scatter about a nitrate vs. temperature curve $\left(3^{\circ} \mathrm{C}<\mathrm{T}<16^{\circ} \mathrm{C}\right)$ is $\pm 3.4 \mu \mathrm{M}$, and the reproducibility

\footnotetext{
${ }^{8}$ Deep oxygen analyses from January - February, 1986 were systematically higher by $0.4-0.6 \mathrm{ml} \mathrm{l}^{-1}$ than the climatological mean, whereas a nearby Oceanus cruise (P. Brewer, pers. comm.) did not reveal such anomalies. We attribute the anomalies to analytical problems, and hence these oxygen data have not been included in the analysis
} 


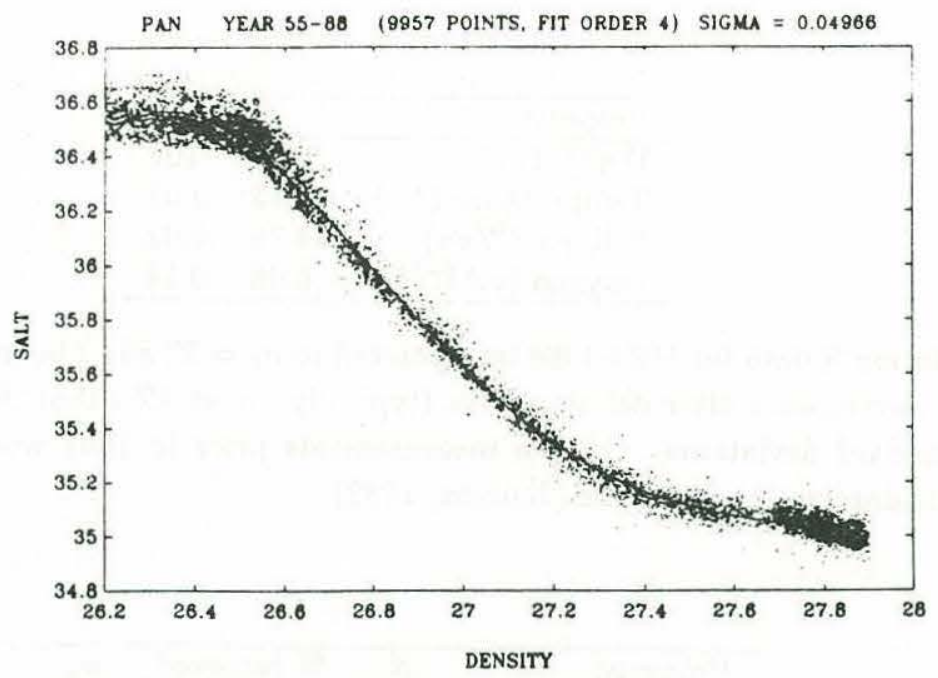

(a)
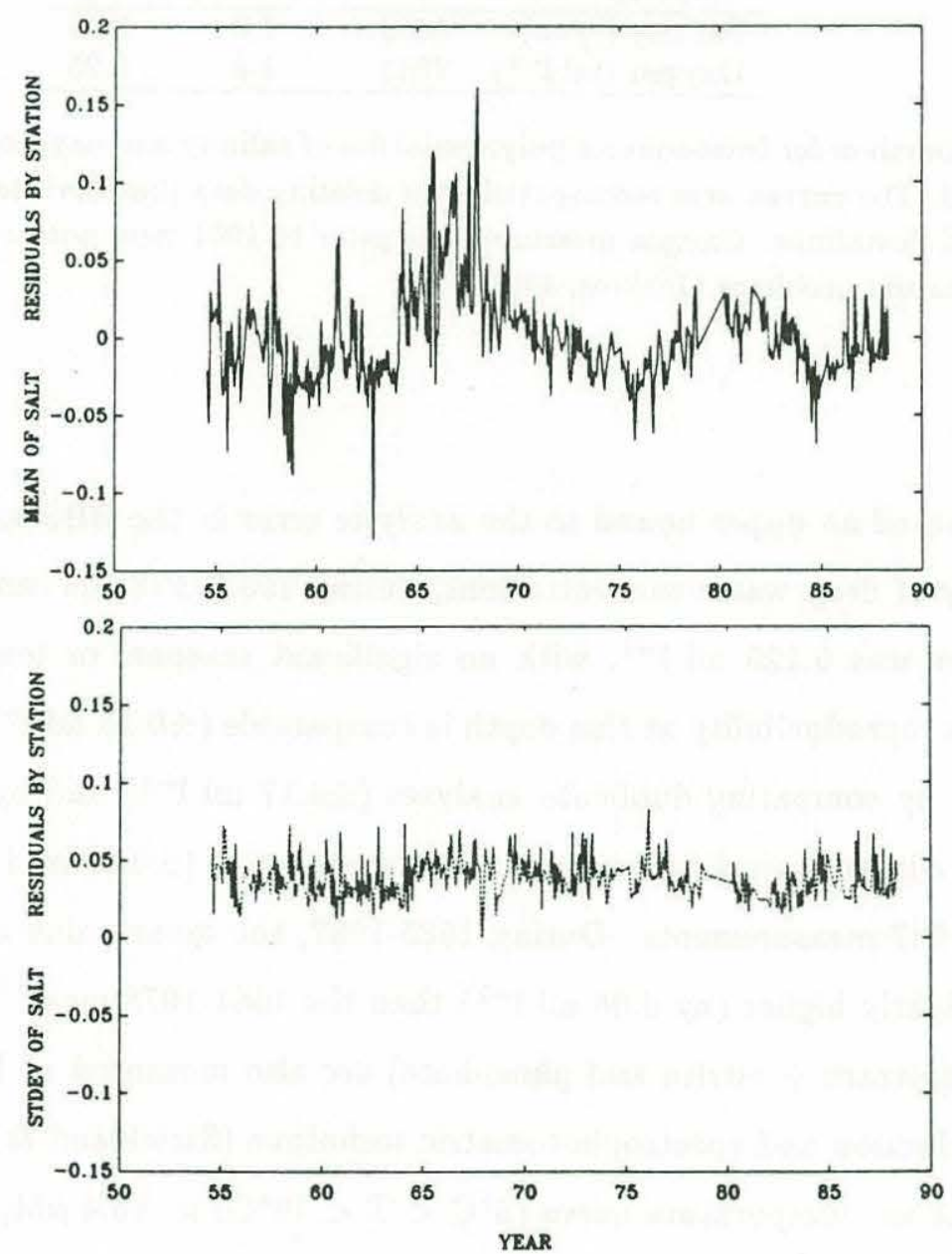

Figure 2-17: Fourth-order least-squares polynomial fits of (a) salinity and (b) oxygen to density over the range $\sigma_{\theta}=26.2-27.9$. The mean residuals from the fitted curves at each station reflect long-term trends (a combination of natural variation and measurement drift). The standard deviations of the residuals at each station will tend to reflect the reproducibility for given station. 

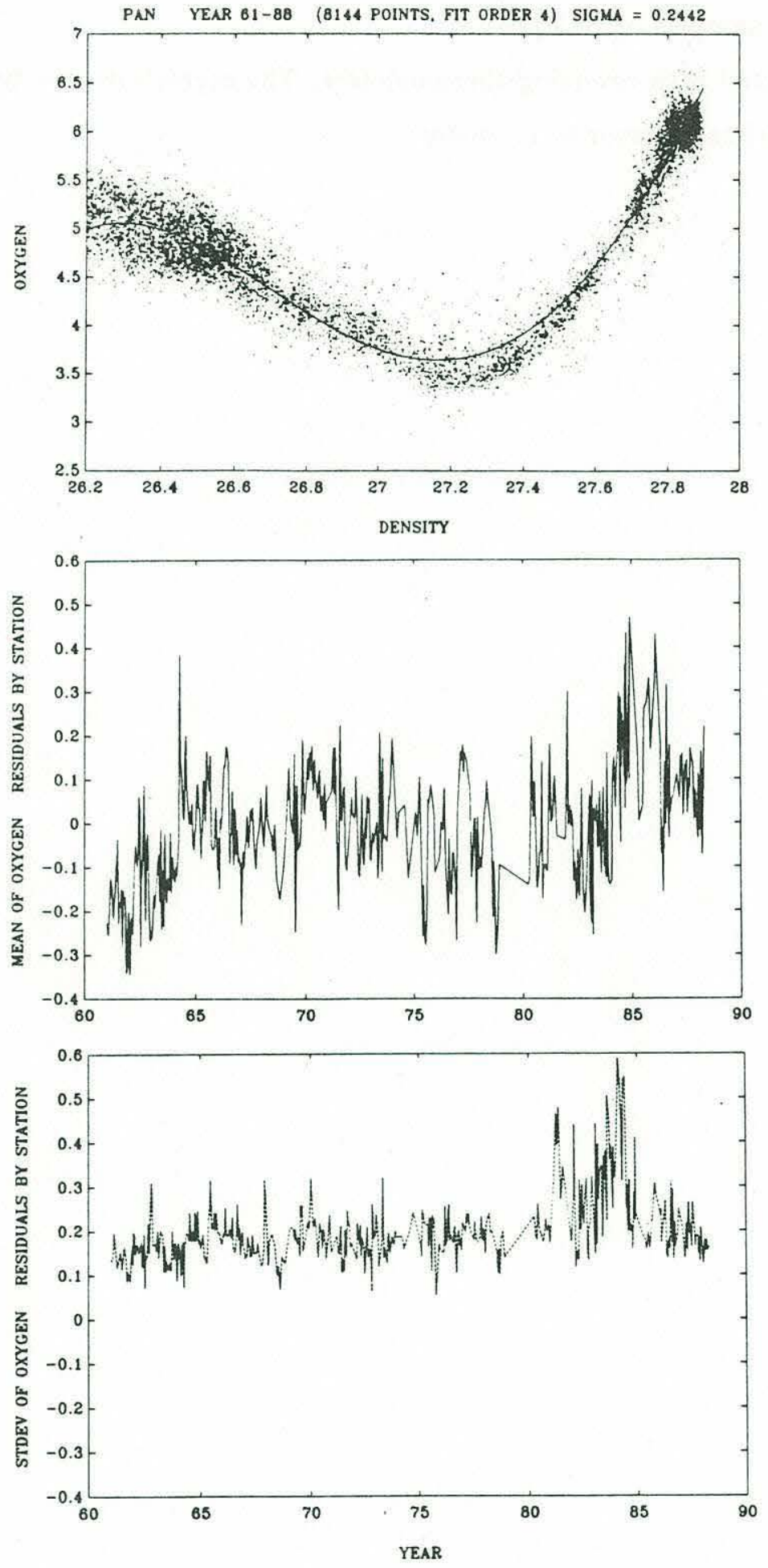

Figure 2-17: (continued) 
of deep (2000 meter) samples is $\pm 3.2 \mu \mathrm{M}$.

Depths are corrected with reversing thermometers. The corrections are typically less than $1 \%$; the maximum estimated error is \pm 5 meters. 


\section{Chapter 3}

\section{Rates of Vertical Mixing, Gas Exchange, and New Production}

\subsection{Introduction}

The development of a summer oxygen maximum in the seasonal thermocline is a common feature of subtropical waters (Schulenberger and Reid, 1981), and has been attributed to "new" photosynthetic production ( op cit; J\&G). However, physical processes such as radiative heating and air injection may produce supersaturation unrelated to biological activity. On the other hand, much of the biogenic oxygen may be lost from the seasonal thermocline via vertical mixing and gas exchange. Measurements of dissolved argon can quantify these effects since argon is an inert gas whose physical properties (e.g. solubility and diffusivity) are close to those of oxygen and have a similar temperature dependence (Benson, 1965). In the summer of 1985, Craig and Hayward (1987) compared oxygen and argon supersaturations at two stations in the subtropical North Pacific, and concluded that most of the excess oxygen was photosynthetically produced since argon was much less supersaturated than oxygen.

Since the solubility of argon has a strong temperature dependence, seasonal heating forces an annual cycle in argon concentration. Argon's inability to "keep up" with the thermal cycle, as measured by its departures from solubility equilibrium, can be used to estimate rates of transport processes in the upper ocean. We will couple measurements of the seasonal cycles of argon, helium, temperature, and oxygen with an upper ocean model (Chou, 1985; Musgrave 
et al., 1988). By linearizing the model's response to parameter variations and then applying an inverse technique (singular value decomposition), we then infer rates of vertical mixing, gas exchange, air injection, and new production.

We have previously demonstrated this approach using data from 1985 (Spitzer \& Jenkins, 1989). Here, we extend it to a three year timeseries (1985-1987), and compare this period to climatological average conditions at Bermuda.

\subsection{Methods}

The results reported here are from a three year study (April 1985 - April 1988) at Station $\mathrm{S}\left(32^{\circ} \mathrm{N} 64^{\circ} \mathrm{W}\right)$, situated 25 kilometers southeast of Bermuda in the Sargasso Sea. We have augmented an ongoing series of biweekly hydrographic stations which measure temperature, salinity and oxygen at standard depths with approximately monthly sampling for nutrients, argon, helium, and tritium and an additional shallow cast to obtain approximately 10 meter resolution in the upper 100 meters.

Samples for argon analysis are collected by gravity-feed from Niskin bottles and stored in copper tubes containing 13 grams of seawater (Weiss, 1968; Jenkins and Clarke, 1976). In the laboratory, the seawater is quantitatively degassed under vacuum, purified to remove contaminant gases, and the argon is then measured by isotope dilution mass spectrometry. Concentrations are measured as (cc at S.T.P.) (g seawater) ${ }^{-1}$. Saturation anomalies $(\Delta \mathrm{Ar})$ are expressed as percent deviations from smoothed gas solubilities compiled by Weiss (1970; 1971a), which have a precision of about $0.2 \%$.

Replicate analyses of Vineyard Sound, Mass. surface water obtained by bucket sampling indicate that the total uncertainty associated with copper tube sampling, storage, extraction and mass spectrometric analysis is $0.3 \%$. Absolute calibrations of the isotopic standards, volumes and temperatures are accurate to $0.15 \%$ and traceable to National Bureau of Standards (NBS). A series of 20 samples from a depth of $2000 \pm 200$ meters show no statistically significant trends with a mean $\Delta \mathrm{Ar}$ of $-0.7 \%$, but have a somewhat larger sample standard deviation of $0.6 \%$. This is an upper bound to the analytical error because it includes any natural variations (presumed to be small at this depth), but may indicate an additional source of error. Laboratory experiments suggest that if the seawater warms up during sample collection, exsolved bubbles 
may occasionally be trapped in the samplers. Extreme conditions created in the laboratory resulted in a $1-2 \%$ increase in mean supersaturations, but more typical conditions yielded a 0.0-0.2\% bias. Recent measurements indicate that this problem can be minimized with careful sampling. In total, however, the analytical error is small compared to the seasonal range of argon supersaturations (about $3-4 \%$ ).

Helium samples are collected in copper tube samplers containing 45 grams of seawater, and the total helium content is measured by peak height manometry to a precision of $0.2 \%$ (Lott and Jenkins, 1984). Concentrations are measured as (cc at S.T.P.) (g seawater) ${ }^{-1}$. Saturation anomalies $(\Delta \mathrm{He})$ are expressed as percent deviations from smoothed gas solubilities compiled by Weiss (1971b), which have a precision of $0.3 \%$ and maximum systematic uncertainty of $0.5 \%$. Helium data is available only for 1985-1986; measurements for 1987 await refinement of a technique to simultaneously measure neon on the same sample.

Since the seasonal cycle of oxygen will be used to infer rates of new production, the analytical error in the oxygen measurements must also be assessed. Oxygen is determined by modified Winkler titration (Carritt and Carpenter, 1966) at the Bermuda Biological Station (BBS). J\&G estimated an upper bound to the analytic error in the BBS oxygen analyses from the reproducibility of deep water concentrations; during 1961-1978 the sample standard deviation at 2000 meters was $0.125 \mathrm{ml} \mathrm{l}^{-1}$, with no statistically significant seasonal or long-term trends. During 1985-1987, the reproducibility at this depth is comparable $\left( \pm 0.15 \mathrm{ml} \mathrm{l}^{-1}\right)$ and similar estimates were obtained by comparing duplicate analyses $\left( \pm 0.17 \mathrm{ml} \mathrm{l}^{-1}\right)$ and by computing the scatter about a mean climatological oxygen vs. temperature curve $\left( \pm 0.19 \mathrm{ml} \mathrm{l}^{-1}\right.$ for $3^{\circ} \mathrm{C}<\mathrm{T}<$ $16^{\circ} \mathrm{C}$ ) for the $1985-1987$ measurements. In $1985-1987$, the mean value at 2000 meters was 6.12 $\mathrm{ml}^{-1}$, just slightly higher (by $0.06 \mathrm{ml}^{-1}$ ) than the $1961-1978$ mean ${ }^{1}$.

\subsection{Results}

The time series measurements are presented in Figure 3-1 and in Appendix A. The seasonal cycle of temperature at Bermuda (Figure 3-1a) is characterized by deep convection in the late

\footnotetext{
${ }^{1}$ Deep oxygen analyses from January - February, 1986 were systematically higher by $0.4-0.6 \mathrm{ml} \mathrm{l}^{-1}$ than the climatological mean, whereas a nearby Oceanus cruise (P. Brewer, pers. comm.) did not reveal such anomalies. We attribute the anomalies to analytical problems, and hence these oxygen data have not been included in the analysis
} 
winter, followed by increasing stratification during spring and summer, and finally erosion of the seasonal thermocline in the fall. The seasonal cycle of argon concentration (Figure 3-1b) is qualitatively similar to that of temperature, since the argon cycle is controlled by the strong temperature dependence of argon solubility.

By comparing the observed argon concentrations to those expected from solubility equilibrium, thermal effects can be separated from other physical processes. The seasonal cycle in percent argon supersaturation in the upper 200 meters is shown in Figure 3-1c, with the mixed layer history presented in more detail in Figure 3-2a. In late winter, argon supersaturation in the deep mixed layer reflects a dynamic balance between air injection and gas exchange. In spring and summer, the mixed layer supersaturation increases because outgassing can remove only a fraction of the supersaturation produced by radiative heating, air injection, and the flux of argon from below. In the seasonal thermocline, radiative heating sustains a subsurface maximum in argon supersaturation despite vertical mixing. In the fall, cooling and convective mixing erode the subsurface maximum and gas exchange erases the remnant supersaturation in the mixed layer. Rapid gas exchange and air injection "recharge" argon concentrations to their winter-time values.

The seasonal cycle of percent oxygen supersaturation (Figure 3-1d) is qualitatively similar to that of argon down to the base of the euphotic zone, below which respiration produces oxygen undersaturation. However, the seasonal amplitude is much larger because oxygen is biologically produced in situ, whereas the argon cycle is driven solely by physical processes. The seasonal cycle of oxygen at Bermuda, discussed by Jenkins and Goldman (1985), is characterized by near saturation values in the deep winter mixed layer with some slight undersaturation due to rapid cooling and the upward mixing of oxygen depleted water from below. During spring and summer, a subsurface oxygen maximum develops in the seasonal thermocline, accompanied by a "counterpoint" pattern of undersaturation below due to respiration. Finally, fall cooling and convective mixing lead to erasure of the subsurface extrema. 
(a)

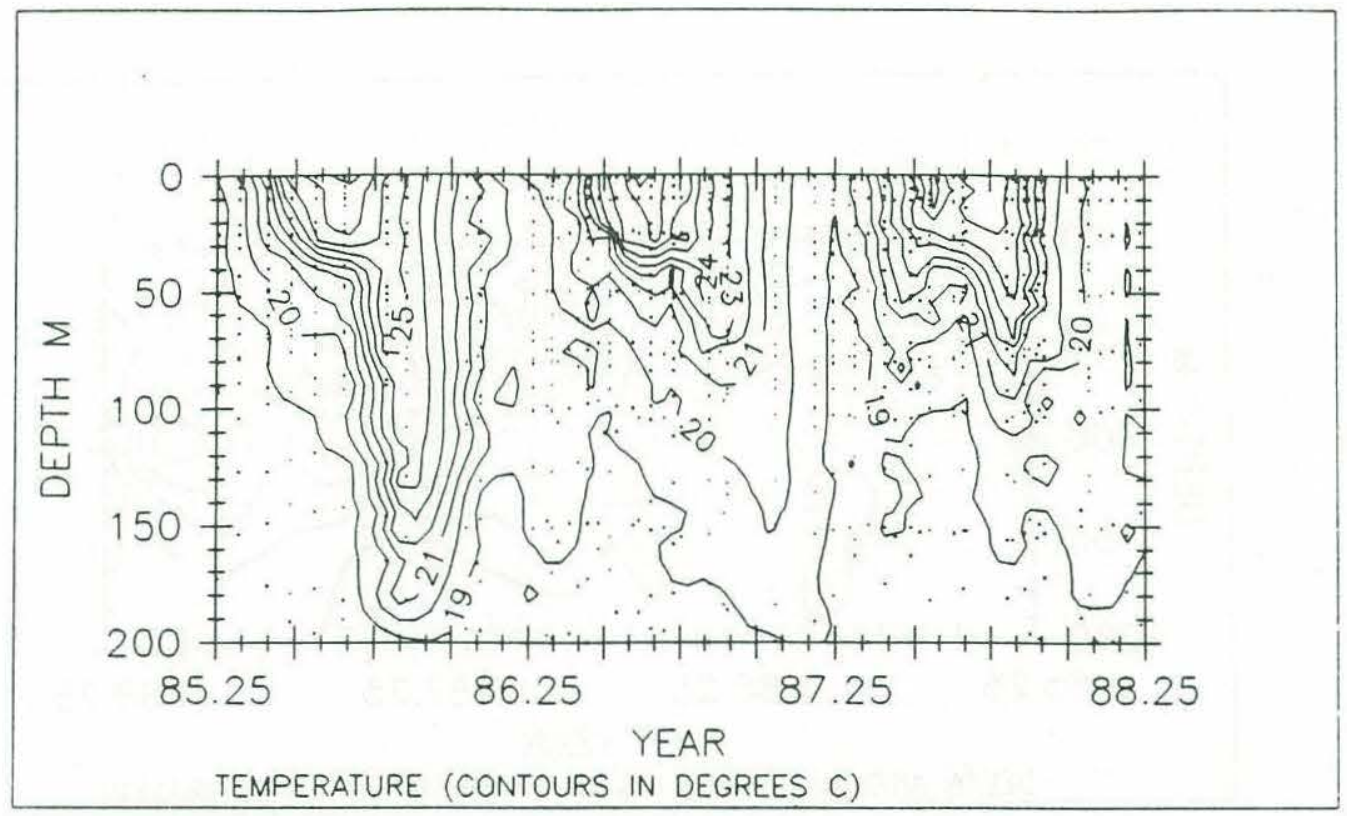

(b)

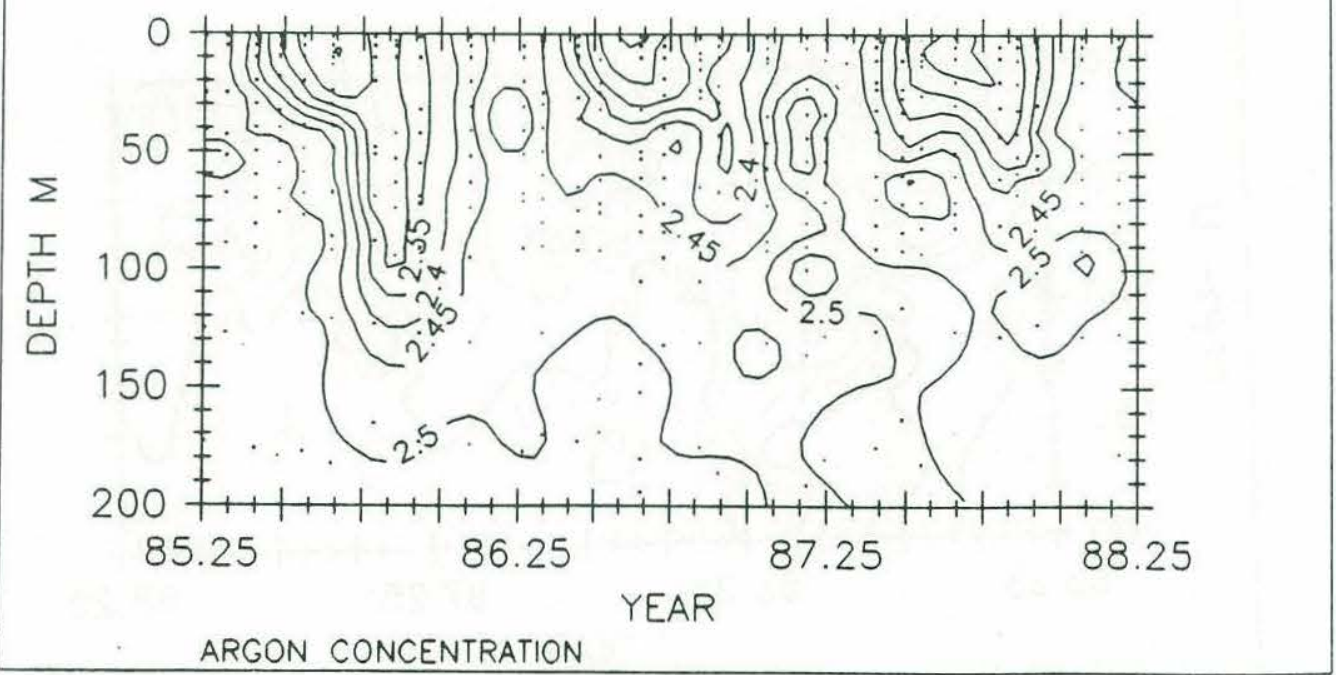

Figure 3-1: $\quad$ Seasonal distributions for April 1985 - April 1988 of: (a) temperature $\left({ }^{\circ} \mathrm{C}\right)$, (b) argon concentration $\left(10^{-4} \mathrm{cc} \mathrm{g}^{-1}\right),(\mathrm{c}) \Delta \mathrm{Ar}$ (percent supersaturation), and (d) $\Delta \mathrm{O}_{2}$ (percent supersaturation). Sample locations are indicated by crosses. Year-time is computed as the fraction of the year elapsed since January 1 (e.g. 85.50 is equivalent to July 1, 1985). The argon distribution is driven by seasonal heating, as seen from the similarity to the thermal cycle. Oxygen is controlled only partly by physical processes, hence the amplitude of its seasonal cycle is larger than that of argon. 
(c)

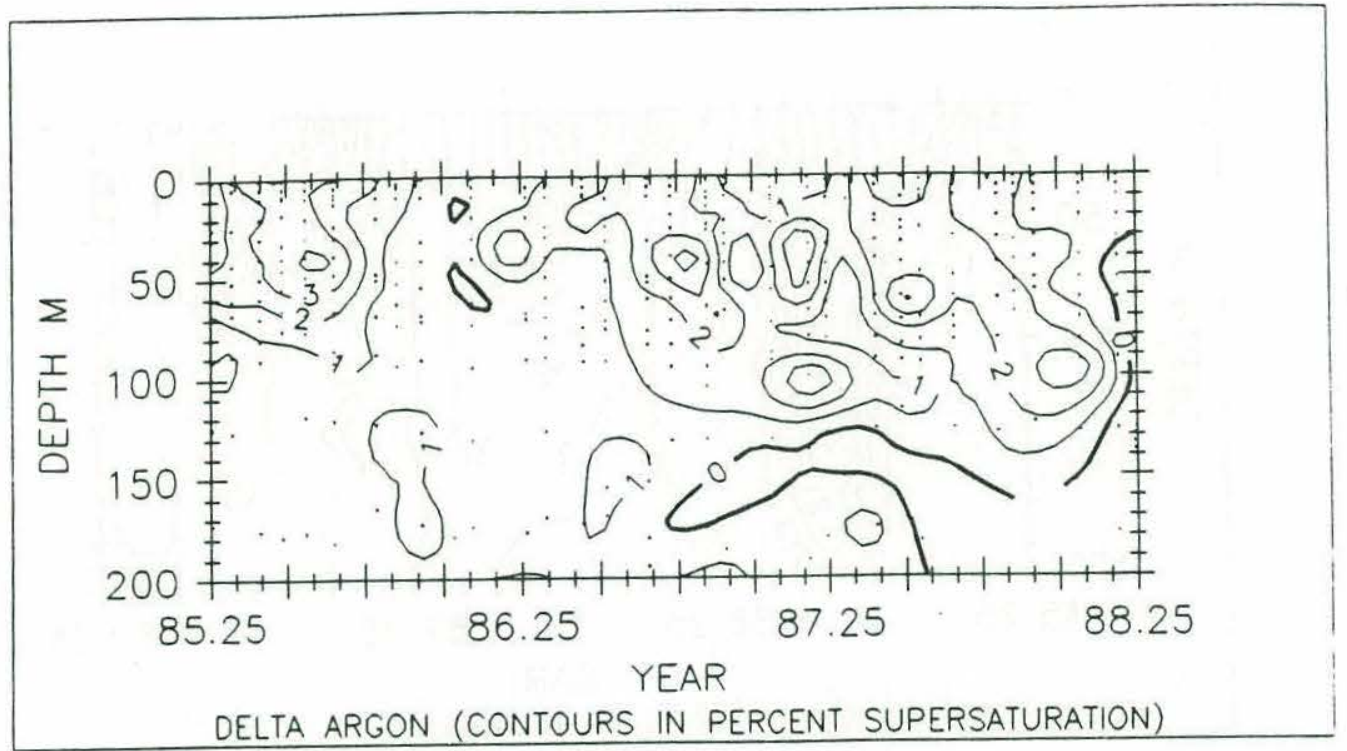

(d)

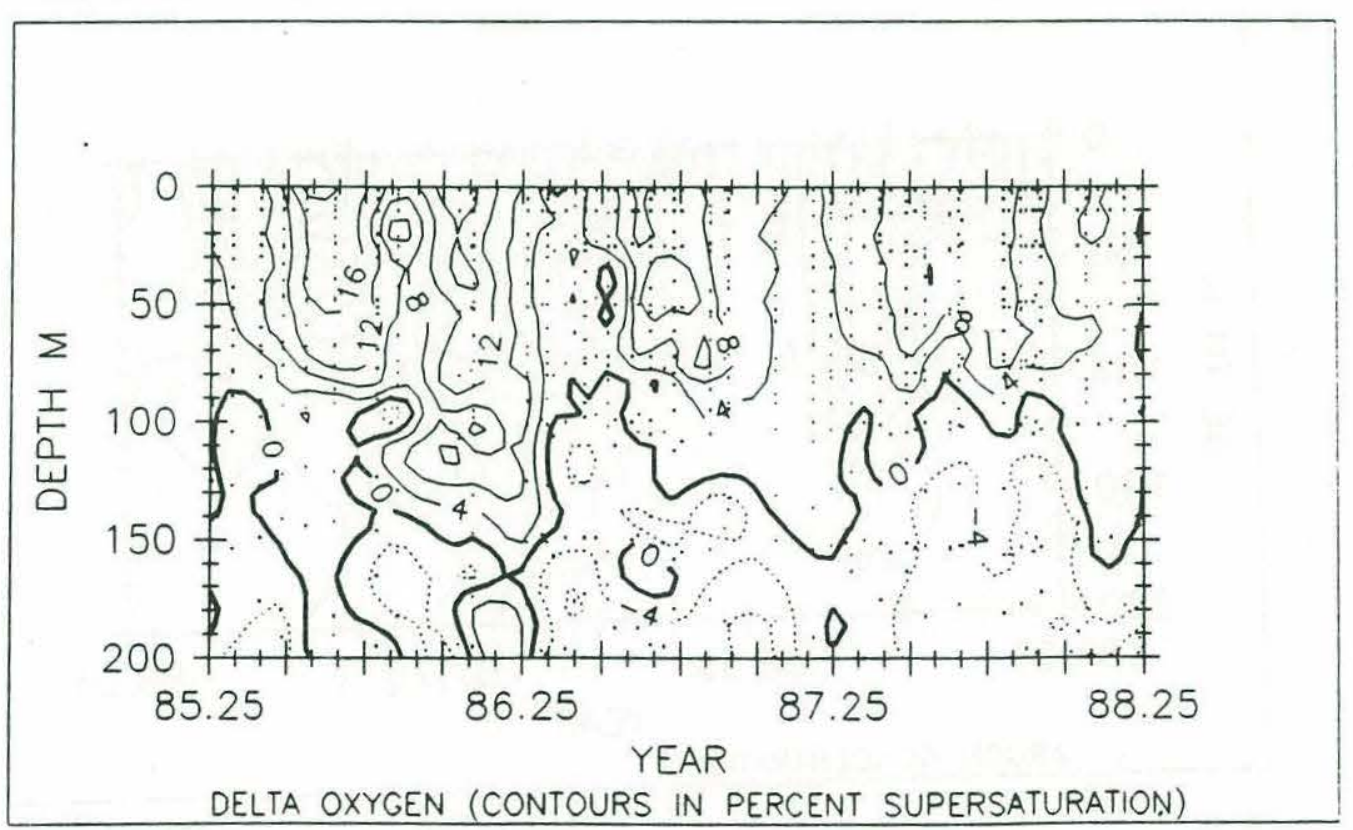

Figure 3-1: $\quad$ (continued) 
(a)

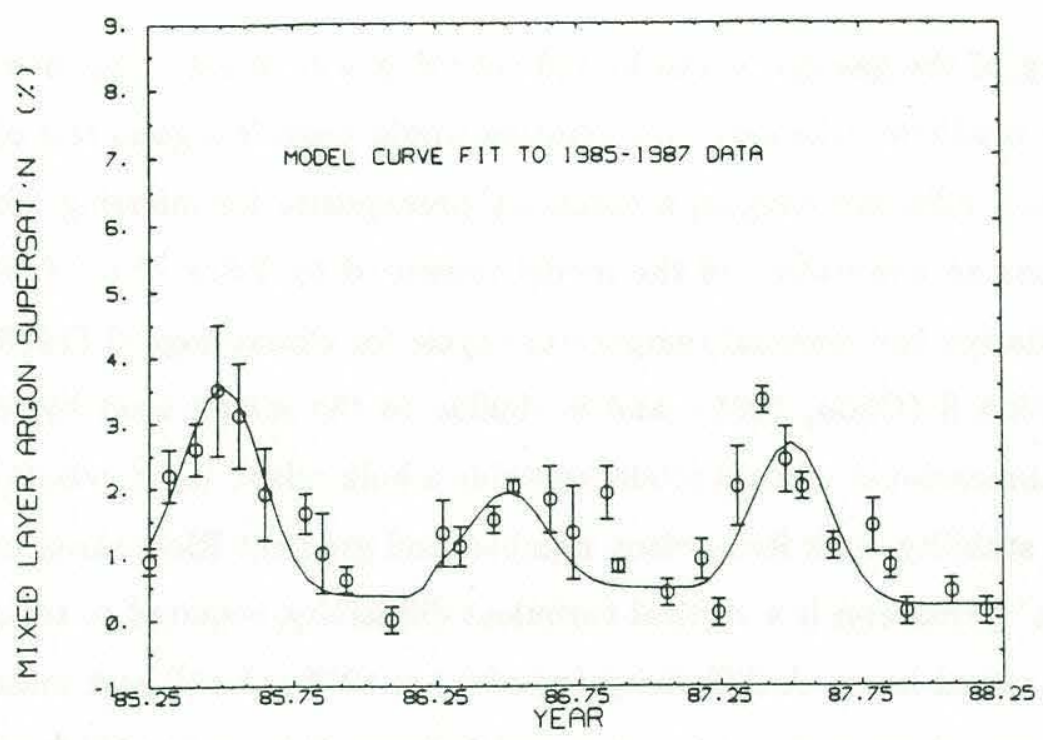

\section{ARGON}

(b)

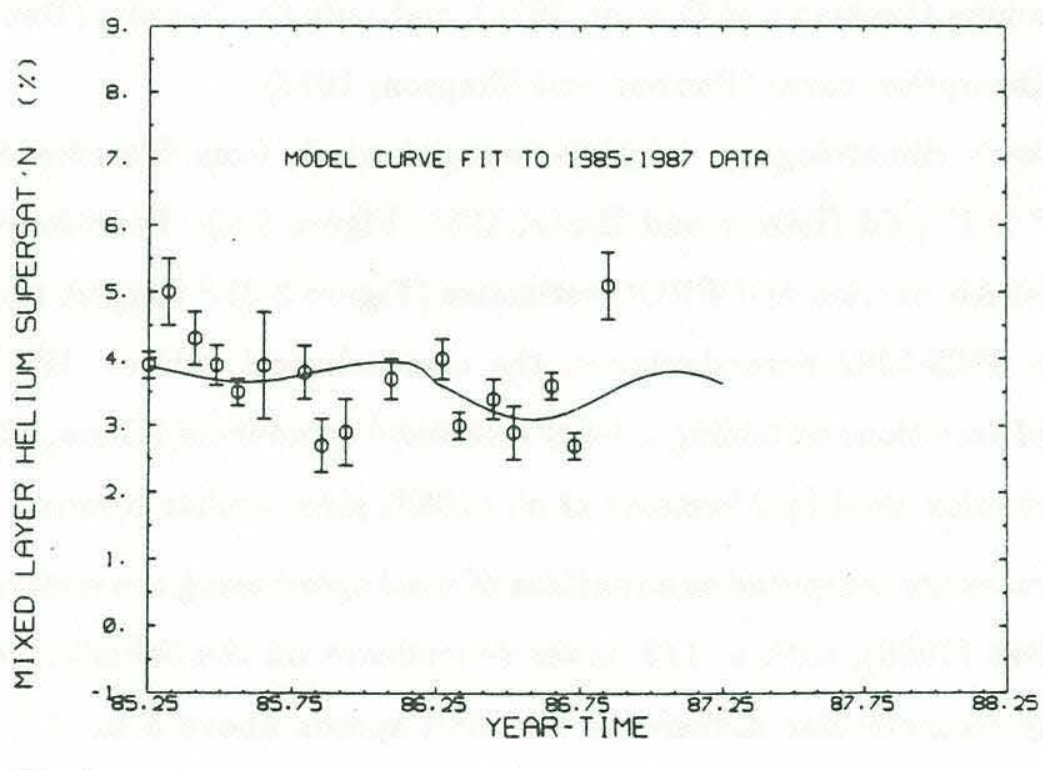

HEL IUM

Figure 3-2: Seasonal cycle of supersaturation of (a) argon and (b) helium for 1985-1987, where each data point represents an average of several samples within the mixed layer (mixed layer depth determined using a $\Delta \mathrm{T}=0.5^{\circ} \mathrm{C}$ criterion) and the error bars are statistical uncertainties associated with the mean value. Solid lines are model fits using non-linear least-squares (see text for details). The argon cycle differs from that of helium because it is driven mostly by radiative heating rather than air injection. 


\subsection{A Seasonal Upper Ocean Model}

Our understanding of the gas cycles can be refined using a seasonal upper ocean model. The model's success in predicting the argon distribution would provide a good test of its simulation of physical processes affecting oxygen, a necessary prerequisite for inferring biological oxygen production. We use an adaptation of the model presented by Price et al. (1986), which has succeeded in simulating the seasonal temperature cycle for climatological (1960-1970 average) conditions at Station S (Chou, 1985), and is similar to the model used by Musgrave et al. (1988). This one-dimensional vertical model contains a bulk mixed layer, whose depth is determined from static stability, bulk Richardson number, and gradient Richardson number criteria. The only "tunable" parameter is a vertical turbulent diffusivity, required to transport heat and tracers below the mixed layer. A diffusivity $(\kappa)$ of $0.1 \times 10^{-4} \mathrm{~m}^{2} \mathrm{~s}^{-1}$ best matches the climatological mean temperature cycle, and was varied between $0.7-1.7 \times 10^{-4} \mathrm{~m}^{2} \mathrm{~s}^{-1}$ in model runs. The model is forced by seasonally-modulated climatological values for heat flux (Bunker, 1975), Ekman pumping (Leetma and Bunker, 1978), and radiative heating (Bunker, 1975) with a Jerlov type IA absorption curve (Paulson and Simpson, 1977).

We used Bunker's climatological monthly-averaged winds from Marsden Square 115, interpolated to a $1^{\circ} \times 1^{\circ}$ grid (Isemer and Hasse, 1985; Figure 3-4). Land-based records from the Bermuda Naval Air Station and FNOC estimates (Figure 3-3) ${ }^{2}$ suggest that the monthlyaveraged winds in 1985-1987 were similar to the climatological values. We have simulated shorter period wind variations by adding a 4-day sinusoidal component (Chou, 1985). A stochastic wind parametrization used by Musgrave et al. (1988) gives similar results.

Gas exchange rates are computed as a function of wind speed using the relationship proposed by Liss and Merlivat (1986), with a $-1 / 2$ power dependence on the Schmidt number (ratio of kinematic viscosity to molecular diffusivity) for wind speeds above $5 \mathrm{~m} \mathrm{~s}^{-1}$. A $-2 / 3$ power dependence is used for lower wind speeds, in accordance with Deacon's (1977) model. Regression statistics of the field measurements of piston velocity as a function of wind speed suggest an uncertainty of about $\pm 15 \%$ over the domain of interest. In model runs, the gas exchange rate

\footnotetext{
${ }^{2}$ These estimates are obtained by the Fleet Numerical Oceanographic Center of the U.S. Navy by assimilating atmospheric pressure data, ship reports and meteorological buoy data into a numerical geostrophic model of the lower atmosphere (Lewis \& Grayson, 1972; Friehe \& Pazan, 1978; Brink, 1989). George Halliwell at Univ. of Rhode Island, Grad. School of Oceanography kindly provided this data set for 1976-1987.
} 


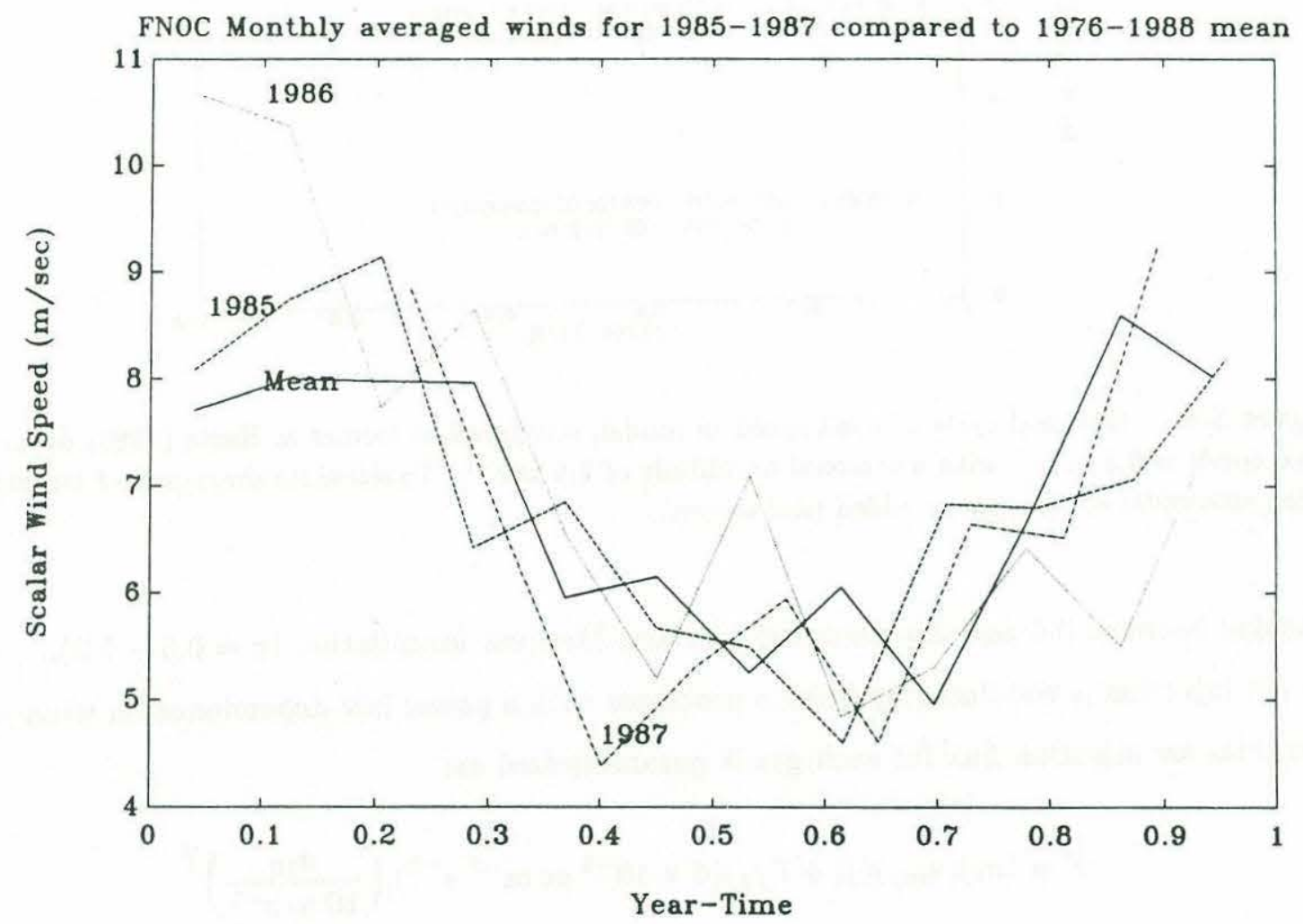

Figure 3-3: Seasonal cycle of wind speed from FNOC estimates for 1985, 1986, 1987, and the 1976-1988 average. 


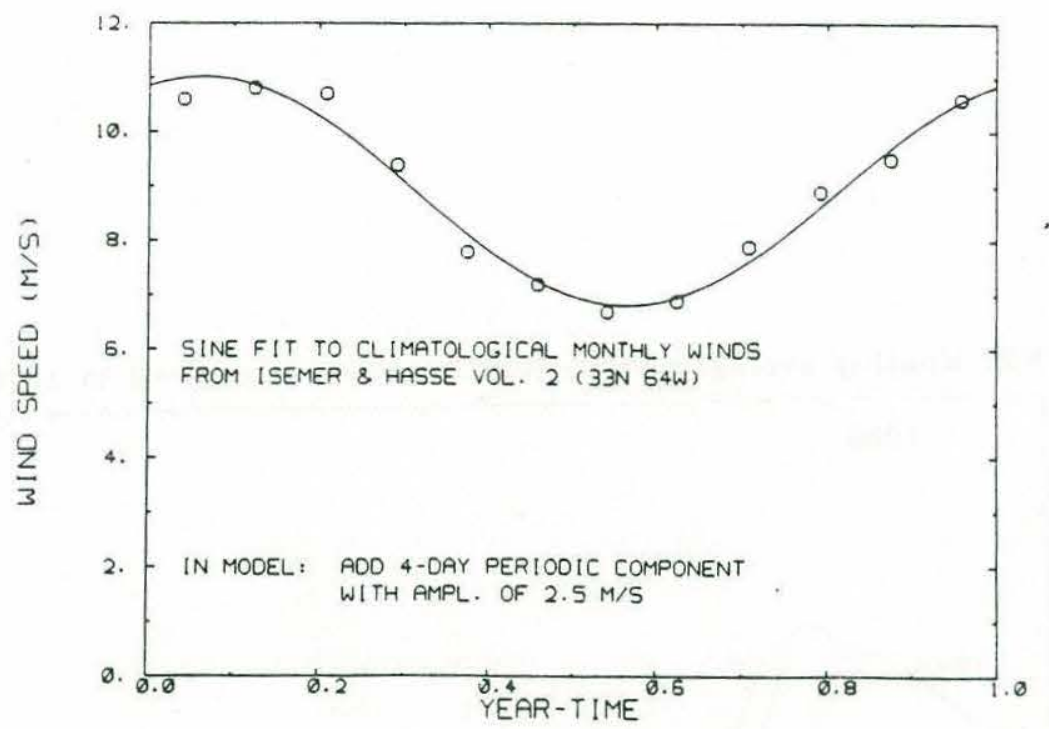

Figure 3-4: Seasonal cycle of wind speed in model, compared to Isemer \& Hasse (1985) data. Mean wind speed is $8.5 \mathrm{~m} \mathrm{~s}^{-1}$ with a seasonal amplitude of $2.5 \mathrm{~m} \mathrm{~s}^{-1}$. To simulate short-period variations, a 4-day sinusoidal component is added (not shown).

is varied between 0.5 and 2.0 times the Liss and Merlivat formulation $(g=0.5-2.0)$.

Air injection is simulated by bubble processes with a power law dependence on wind speed. The total air injection flux for each gas is parameterized as:

$$
F=\left(\alpha_{i}\right)\left(a_{i n j}\right)\left(f_{t}+\Gamma f_{p}\right)\left(6 \times 10^{-8} \mathrm{cc} \mathrm{m}^{-2} \mathrm{~s}^{-1}\right)\left(\frac{u_{10}}{10 \mathrm{~m} \mathrm{~s}^{-1}}\right)^{\gamma}
$$

where the flux is measured in (cc at STP) $\mathrm{m}^{-2} \mathrm{~s}^{-1}, \alpha_{i}$ is the mole fraction of the gas in air, and $u_{10}$ is the wind speed at 10 meters above the sea surface $\left(\mathrm{m} \mathrm{s}^{-1}\right)$. Two modes of bubble trapping (total and partial trapping) are used (Jenkins, 1988b), with $f_{t}$ and $f_{p}$ as the relative fractions of each component constrained by $f_{t}+f_{p}=1$. The air injection flux is normalized to that for helium at $20^{\circ} \mathrm{C}$ by using a normalization factor $(\Gamma)$ associated with the diffusive limitation for gas transfer from the bubbles given by:

$$
\Gamma=\left(D_{i} / D_{o}\right)^{2 / 3}
$$

where $D_{o}$ is the molecular diffusivity of helium at $20^{\circ} \mathrm{C}$. In model runs, the air injection amplitude $\left(a_{i n j}\right)$ was varied from $0.0-1.0$, the wind speed exponent $(\gamma)$ was varied from 1.0-3.5, and 
the fraction of total trapping $\left(f_{t}\right)$ was varied between 0.0-1.0.

An additional effect is variation in barometric pressure. From Isemer and Hasse (1985), the mean sea level pressure at Station $\mathrm{S}$ is $1018.5 \mathrm{Hpa}$, with a seasonal amplitude of $\pm 2.5 \mathrm{Hpa}$. Although we have used 1 atmosphere $(1013.25 \mathrm{HPa})$ in calculating measured supersaturations, we must account for the actual barometric pressure in the model by increasing the effective solubility by $0.3 \%$ in winter and $0.7 \%$ in summer. The seasonal variation in barometric pressure enhances the rate of ingassing, especially in the summertime.

New production is implemented using two regions of oxygen production, one in the seasonal thermocline and the other near the surface. In the seasonal thermocline, the depth distribution of new production is represented as a truncated sine curve $(0<\theta<\pi / 2)$ between 25 and 75 meters with a maximum at 50 meters (Figure 3-5). Although the true depth dependence is unknown, this parameterization tends to give a conservative estimate of the required oxygen production (Chou, 1985; Musgrave et al, 1988). In model runs, we have varied the depth of maximum production between 25-100 meters. Near-surface production is specified as a constant value in the upper 5 meters, which is then distributed throughout the mixed layer. Both production terms are seasonally modulated with a maximum in production in early spring (Chou, 1985; Musgrave et al, 1988). The annual oxygen production in the seasonal thermocline $\left(P_{50}\right)$ and in the mixed layer $\left(P_{m l}\right)$ were each varied between $0-10$ moles $\mathrm{m}^{-2} \mathrm{yr}^{-1}$. Below 100 meters, the depth-integrated respiration balances the total production above. The consumption rate is assumed to decrease linearly with depth to 300 meters (the maximum depth in the model), which approximates the form of measured oxygen utilization rates (Jenkins, 1987).

\subsection{Linearization of Model Response}

The features associated with the seasonal cycles in temperature, argon, and oxygen are dependent on several physical (and for oxygen, biological) processes. Results from a sample model run (Figure 3-6) demonstrate that the model simulates these features qualitatively, but the quantitative agreement depends on the precise choice of model parameters. We ran the model over a range of parameters for each process (vertical mixing, gas exchange, air injection, and productivity), and then linearized the model responses in terms of "indices" that could be cal- 


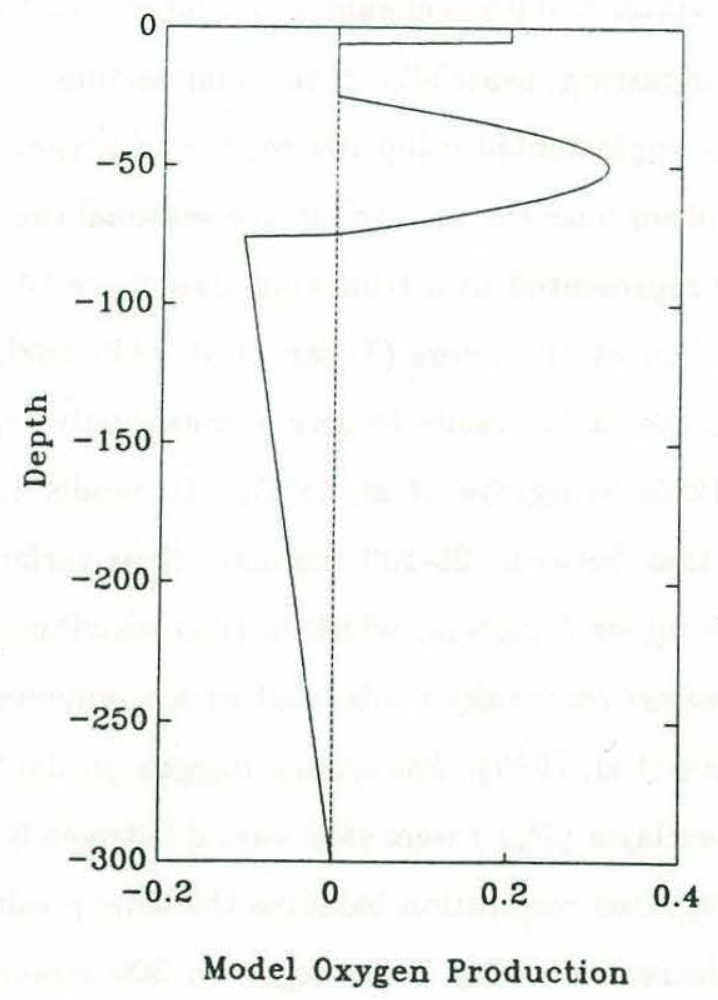

Figure 3-5: Model oxygen production vs. depth, indicating near-surface production and subsurface production with a maximum at 50 meters. 
culated from the data. These indices were selected to be diagnostic for the processes controlling the tracer distributions. The functional forms of these equations were chosen empirically to best match the model responses, and were adapted from results of simple box model calculations. Uncertainties cited with each diagnostic equation (see also Table 1) represent the error associated with linearizing the model response over the parameter range explored. We then used an inverse technique (singular value decomposition) to calculate the set of model parameters that best fit the data.

\subsubsection{Vertical mixing}

Since seasonal heating drives the argon cycle, it is critical to accurately represent the thermal cycle. The only adjustable model parameter which affects temperature is the vertical diffusivity. If vertical mixing is too weak, too much heat is trapped near the surface; if it is too strong, too much heat is transferred below the mixed layer. The strongest constraint on the diffusivity is the mixed layer temperature difference between winter and summer (Figure 3-7), for which we obtain:

$$
\Delta T_{m l}[s-w]=6.31+2.47\left(10^{-4} / \kappa\right) \pm 0.2^{\circ} \mathrm{C}
$$

where the diffusivity $\kappa$ is measured in $\mathrm{m}^{2} \mathrm{~s}^{-1}$. The degree to which the subsurface argon concentration lags behind the in situ temperature increase between winter and summer is also sensitive to vertical exchange in the seasonal thermocline. In the absence of vertical exchange, the observed seasonal warming at a depth of 50 meters would produce an argon supersaturation of $8 \%$. Since the observed supersaturation is much smaller, the removal of argon by vertical transport (and subsequent gas exchange) is significant.

The model predicts that the maximum argon supersaturation in the summer thermocline $\left(\Delta \operatorname{Ar}_{\max }[s]\right)$ is affected not only by the vertical diffusivity $(\kappa)$, but also by the gas exchange rate $(g)$, air injection amplitude $\left(a_{i n j}\right)$ and trapping mode fraction $\left(f_{t}\right)$ :

$$
\Delta \operatorname{Ar}_{\text {max }}[s]=0.76\left(10^{-4} / k\right)+2.2(1 / g)+1.7\left(a_{i n j}\right)\left(0.3 f_{t}+0.9\right) \pm 0.1 \%
$$

As the gas exchange rate is reduced, more argon is trapped in the mixed layer and hence the flux from the seasonal thermocline is also reduced. As vertical mixing is reduced, the 
(a)

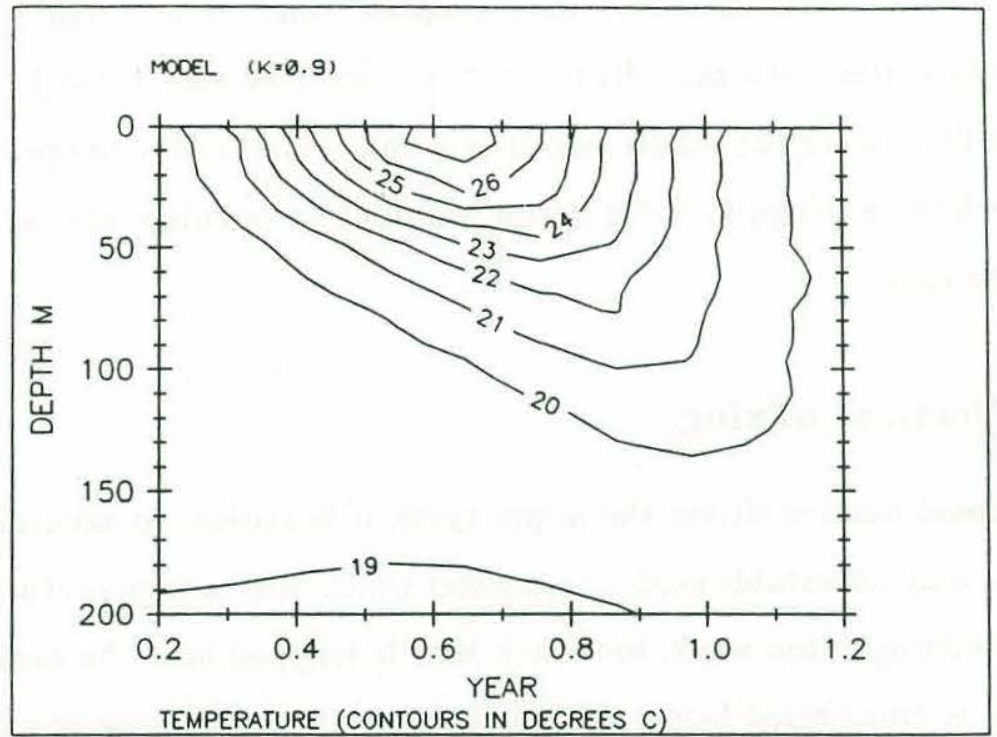

(b)

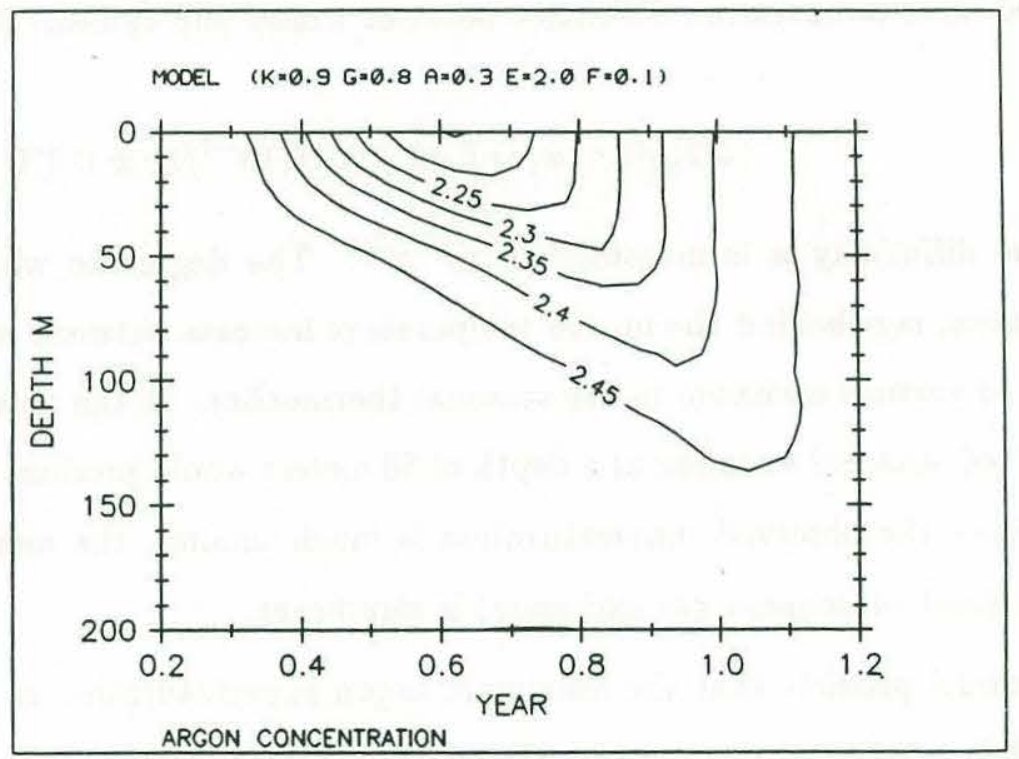

Figure 3-6:

Results of a model run with typical parameters $\left(\kappa=0.9, g=0.8, a_{i n j}=0.3, \gamma=2.0, f_{t}=0.1, P_{m l}=6, P_{50}=4\right)$ for (a) temperature $\left({ }^{\circ} \mathrm{C}\right),(\mathrm{b})$ argon concentration $\left(10^{-4} \mathrm{cc} \mathrm{g}^{-1}\right)$, (c) $\Delta \mathrm{Ar}$ (percent supersaturation), and (d) $\Delta \mathrm{O}_{2}$ (percent supersaturation). Units are the same as in Figure 3-1, but contour intervals differ for $\Delta \mathrm{Ar}$ and $\Delta \mathrm{O}_{2}$. Note that the model simulates the basic features of the data (Figure 3-1). 
(c)

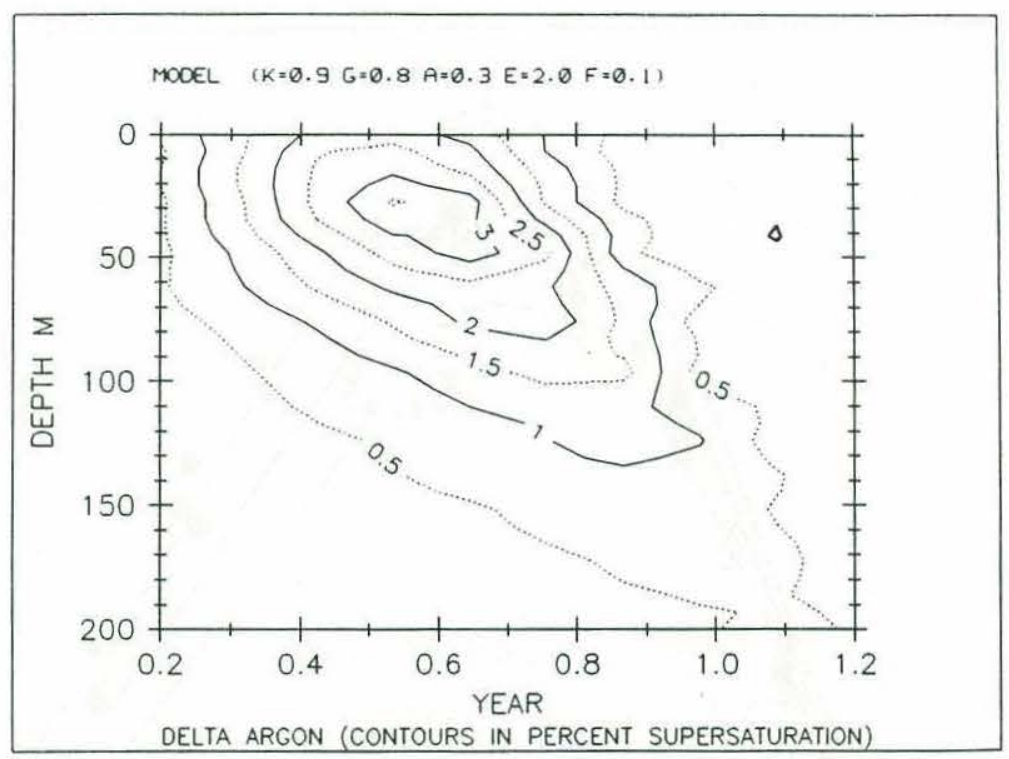

(d)

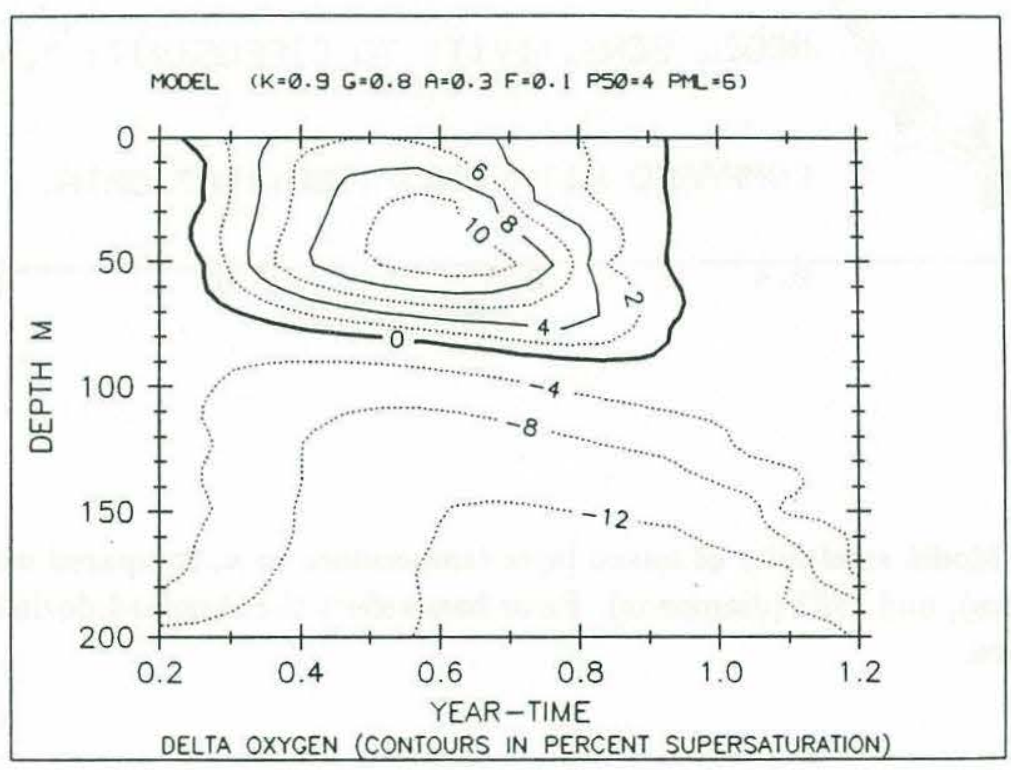

Figure 3-6: (continued) 


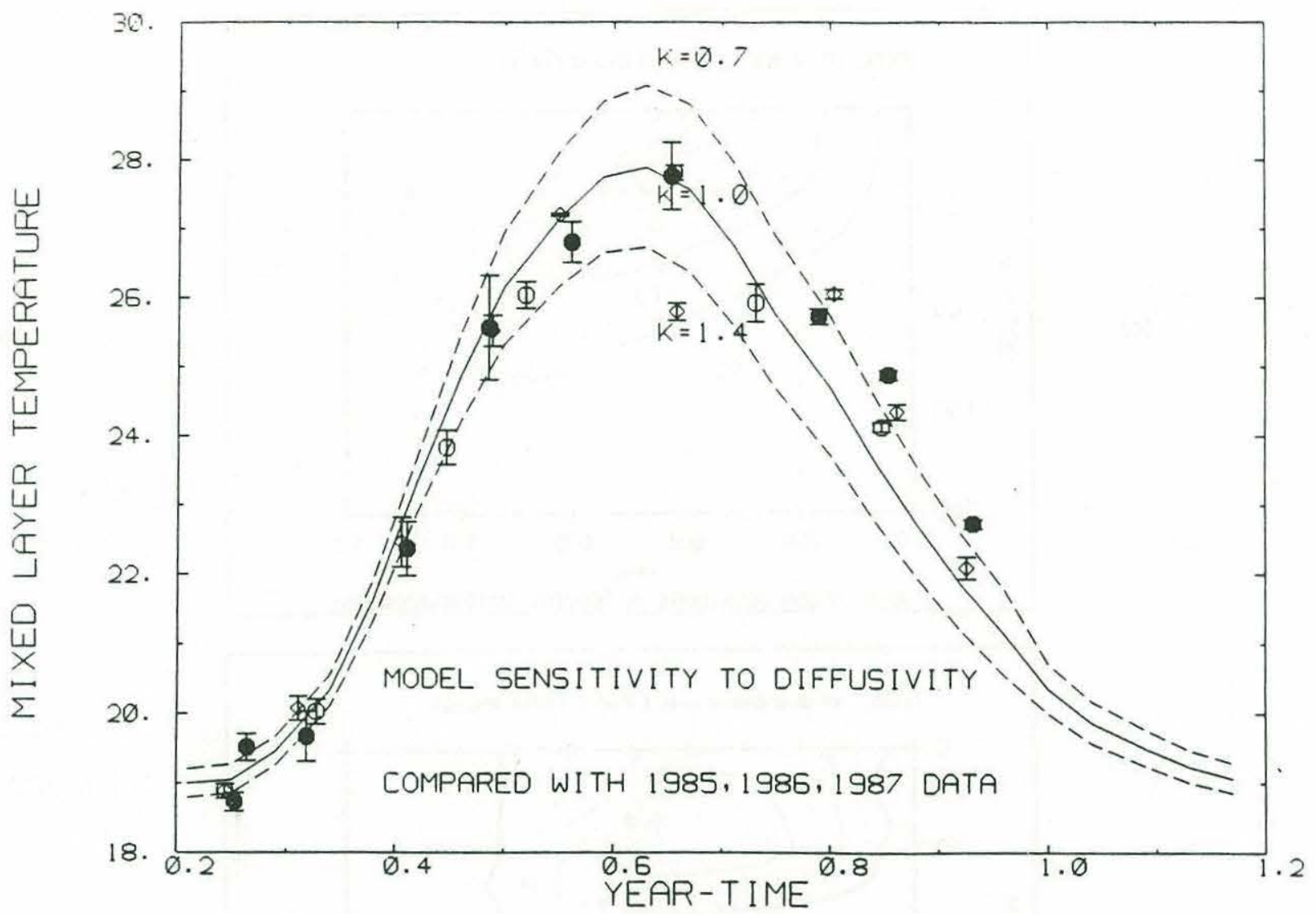

Figure 3-7: Model sensitivity of mixed layer temperature to $\kappa$, compared with 1985 (filled circles), 1986 (open circles), and 1987 (diamonds). Error bars reflect the standard deviation of the mean mixed layer temperature. 
supersaturation increases because the seasonal warming is larger, increasing the "potential" supersaturation ${ }^{3}$. If more bubbles are trapped in the winter mixed layer, this adds to the supersaturation produced by in situ heating during spring and summer. The effective rate of air injection for argon increases with the fraction of total trapping, since this mode is less limited by boundary-layer diffusion ( $c f$. Jenkins, 1988b).

\subsubsection{Gas exchange and air injection}

The degree to which argon approaches solubility equilibrium in the mixed layer depends on the rate of gas exchange, balanced against in situ thermal forcing, the rate of air injection, and the flux of argon from below. Since the upward flux of argon into the mixed layer is much smaller than the gas exchange flux, variations in the rate of vertical mixing have little effect. To some extent, increased air injection is indistinguishable from lower gas exchange rates since both increase the mixed layer supersaturations of argon and helium (Figure 3-8). But, since argon is most sensitive to gas exchange and helium is more sensitive to air injection the two processes can be resolved by using both gases.

As observed in the data, the model predicts that the maximum argon supersaturation in the mixed layer occurs in the summertime. The magnitude of the argon supersaturation in the summer mixed layer $\left(\Delta \mathrm{Ar}_{m l}[s]\right)$ is directly related to the gas exchange rate, and somewhat less sensitive to air injection:

$$
\Delta \operatorname{Ar}_{m l}[s]=(1 / g)\left[2.8\left(a_{i n j}\right)\left(0.2 f_{t}+0.9\right)+1.3\right] \pm 0.1 \%
$$

For a typical model run, air injection contributes about $25 \%$ of the summer supersaturation. However, the winter values $\left(\Delta \mathrm{Ar}_{m l}[w]\right)$ are relatively more sensitive to air injection:

$$
\Delta \operatorname{Ar}_{m l}[w]=(1 / g)\left[2.9\left(a_{i n j}\right)\left(1.0 f_{t}+0.7\right)-1.0\right] \pm 0.1 \%
$$

In the absence of air injection, the model predicts that wintertime cooling leads to undersatu-

\footnotetext{
${ }^{3}$ The mixing of argon across the seasonal thermocline is reduced only slightly since the the decreased diffusivity is mostly compensated by an increase in the concentration gradient. The increase in $\Delta \operatorname{Ar}_{\max }[s]$ can be accounted for mainly by the increase in seasonal warming.
} 
(a)

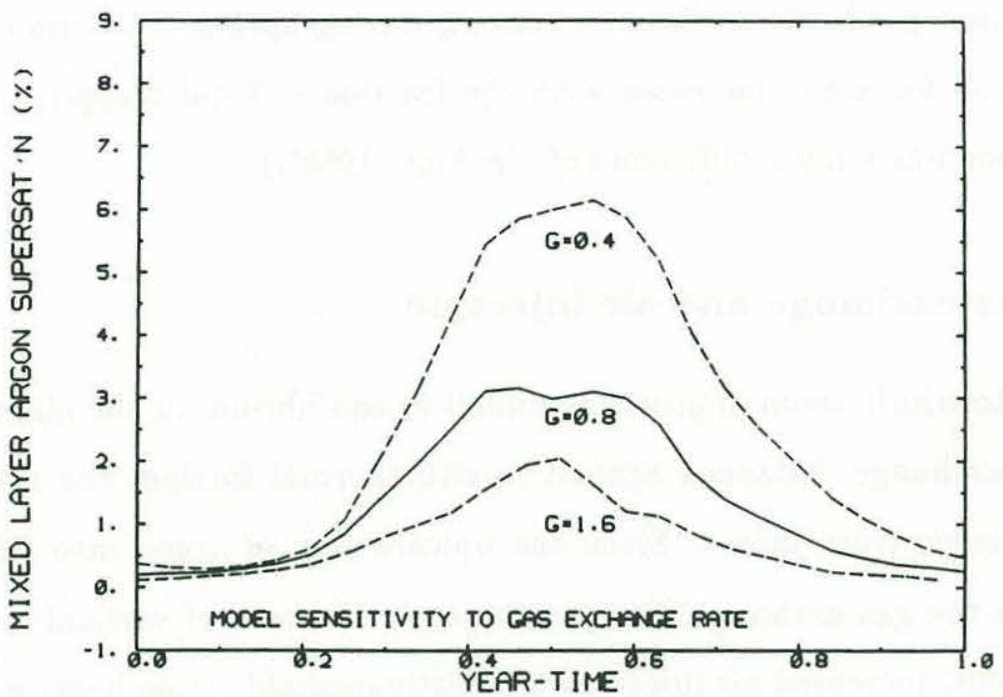

\section{ARGON}

(b)

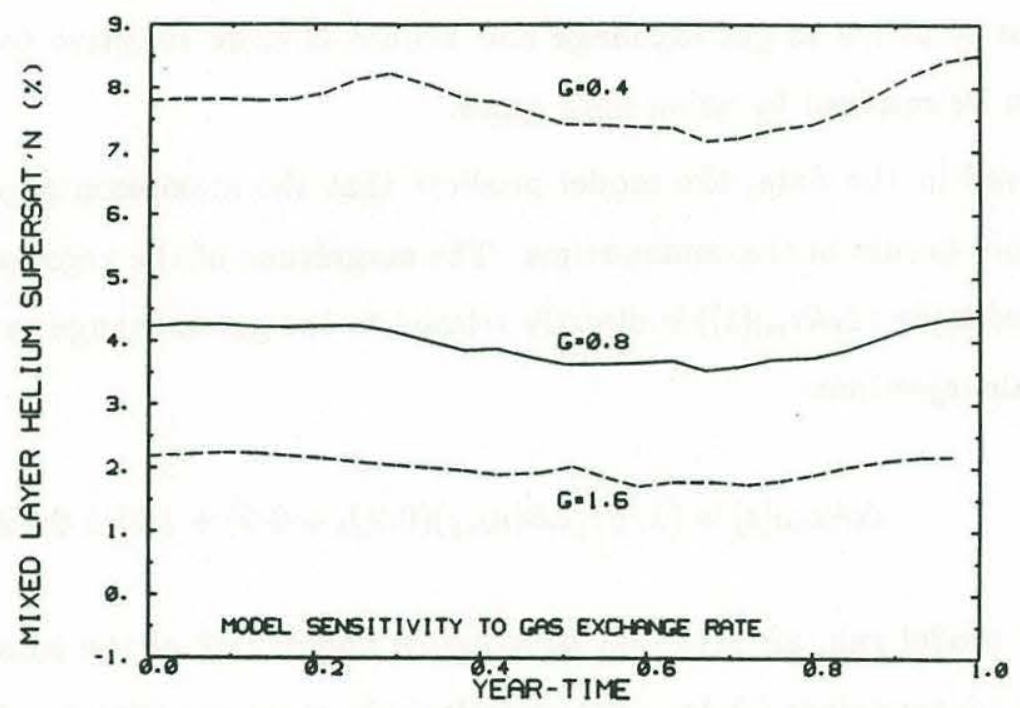

HEL I UM

Figure 3-8: Model sensitivity to variations in gas exchange rate and air injection, using a reference case with $\kappa=0.9, g=0.8, a_{i n j}=0.4, \gamma=2.2, f_{t}=0.1$. The model predicts that mixed layer distributions of (a) $\triangle \mathrm{Ar}$ and (b) $\Delta \mathrm{He}$ are equally sensitive to variations in the gas exchange rate. However, air injection is much less important for (c) $\Delta \mathrm{Ar}$ than for (d) $\Delta \mathrm{He}$. 
(c)

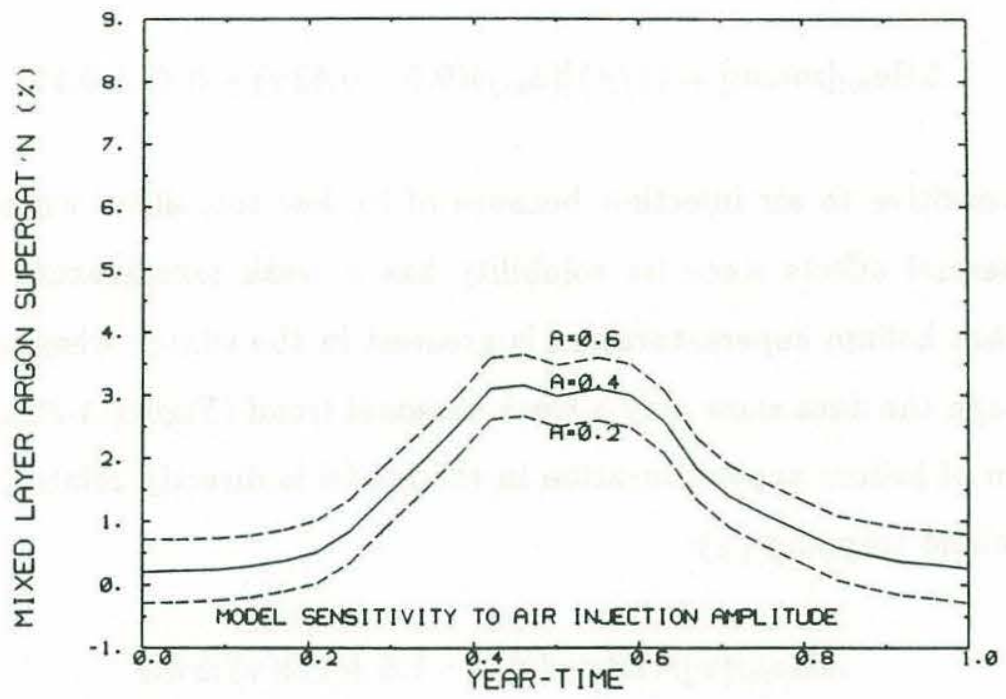

ARGON

(d)

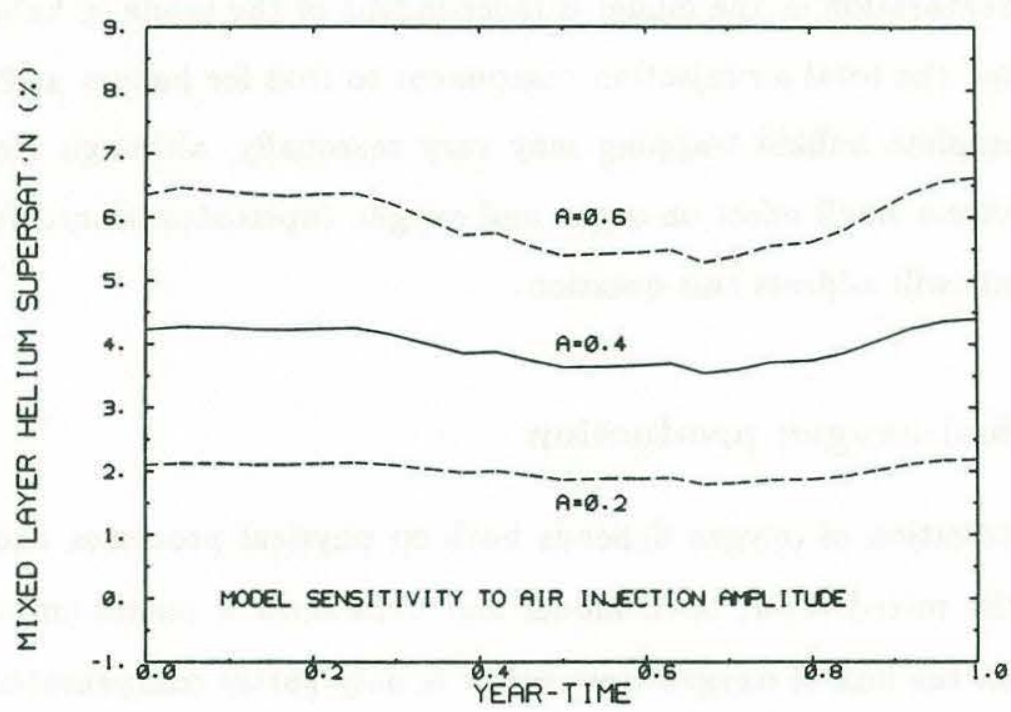

HEL I UM

Figure 3-8: (continued) 
ration of argon in the mixed layer.

For helium, the average supersaturation in the mixed layer $\left(\Delta \mathrm{He}_{m l}[\right.$ mean $\left.]\right)$ is directly related to the gas exchange rate, but also proportional to air injection:

$$
\Delta \mathrm{He}_{\text {ml }}[\text { mean }]=(1 / g)\left[\left(a_{i n j}\right)(9.5-0.63 \gamma)-0.4\right] \pm 0.1 \%
$$

Helium is very sensitive to air injection because of its low solubility, whereas it is relatively insensitive to thermal effects since its solubility has a weak temperature dependence. The model predicts that helium supersaturation is greatest in the winter when air injection is at a maximum, although the data show only a weak seasonal trend (Figure 3-2b). The amplitude of seasonal variation of helium supersaturation in the model is directly related to the wind-speed dependence of bubble trapping $(\gamma)$ :

$$
\Delta \mathrm{He}_{m l}[w] / \Delta \mathrm{He}_{m l}[s]=-1.8+1.2(\gamma) \pm 0.2
$$

The helium supersaturation in the model is independent of the mode of bubble trapping since we have normalized the total air injection component to that for helium at $20^{\circ} \mathrm{C}$. The mixture of partial and complete bubble trapping may vary seasonally, although model runs indicate that this would have a small effect on argon and oxygen supersaturations. Further helium and neon measurements will address this question.

\subsubsection{Biological oxygen production}

The seasonal distribution of oxygen depends both on physical processes and the rates of new production. In the mixed layer, both model and data show a maximum supersaturation in summertime, when the flux of oxygen from below is only partly compensated by gas exchange loss. The model predicts that the winter mixed layer is slightly undersaturated due to entrainment of oxygen depleted water from below the euphotic zone, although the data show a small supersaturation near the surface. The seasonal range in percent oxygen supersaturation is determined mainly by the gas exchange rate, air injection and biological production:

$$
\Delta \mathrm{O}_{2 m l}[s-w]=(1 / g)\left[2.0+0.8\left(a_{i n j}\right)\right] \pm 0.5 \%
$$


This diagnostic minimizes the effects of air injection, and may minimize the impact of any possible systematic offset in the oxygen data. Although the mixed layer supersaturation increases with mixed layer production, the effect is small. As pointed out by Musgrave et al. (1988), this occurs because the gas exchange rate is much faster than the rates of production. Increasing the subsurface production also has an effect, since this will drive more mixing across the seasonal thermocline. Thus, the mixed layer oxygen data provide only a weak constraint on near-surface oxygen production.

In the seasonal thermocline, the model predicts a summer oxygen maximum similar to that observed in the data. The seasonal range in percent oxygen supersaturation is determined mainly by a balance between subsurface biological production and vertical mixing:

$$
\Delta \mathrm{O}_{2 \max }[s-w]=3.7\left(10^{-4} / \kappa\right)+2.2(1 / g)-0.5\left(a_{i n j}\right)+0.2\left(P_{m l}\right)+2.0\left(P_{50}\right)-3.1 \pm 0.2 \%
$$

As subsurface production is increased, the magnitude of subsurface supersaturation increases. If mixed layer production is large enough it will add to the deeper supersaturation maximum by inhibiting the upward mixing flux. Although the magnitude of the supersaturation is determined by the biological production, its time evolution is governed mainly by the physics of mixing and gas exchange, and is quite similar to that for argon (Figure 3-9.

For oxygen, vertical mixing is more important than for argon since biological production creates larger gradients and hence higher fluxes. Thus the oxygen remaining in the seasonal thermocline may represent only a small fraction of that actually produced by photosynthesis

during spring and summer. In this respect argon is not a perfect analog for oxygen, and the model is required to make quantitative inferences.

\subsection{Quantitative Estimates}

The above relationships form a set of empirically-derived equations that constrain the model parameters in terms of observed temperature and gas cycles. The non-linear terms in the equations can be approximated with first-order Taylor series expansions, which introduce little additional error over the domain of interest. Since there are more equations (8) than unknowns (7), the resulting system of linear equations is overdetermined. Thus, it is conceivable that 


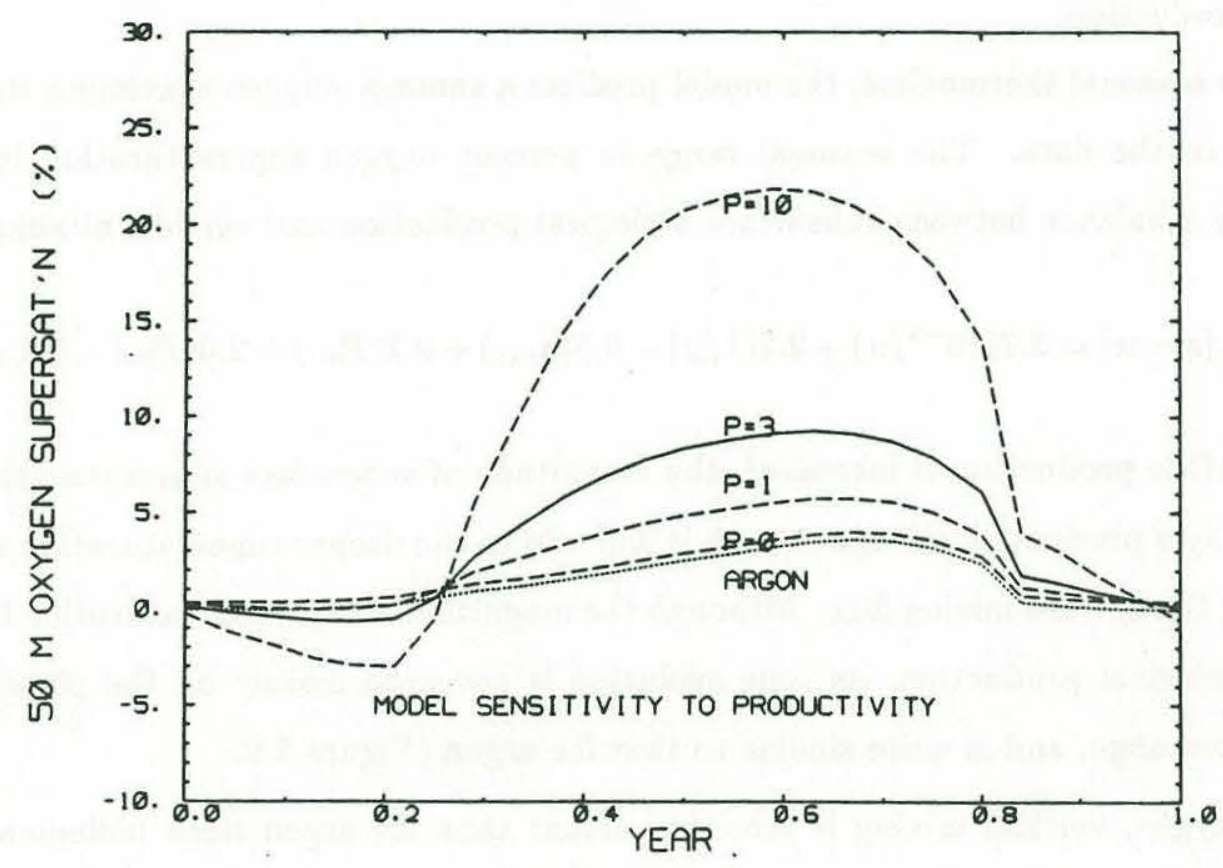

Figure 3-9: Model sensitivity of oxygen supersaturation at 50 meters to biological production for 0-10 moles $\mathrm{O}_{2} \mathrm{~m}^{2} \mathrm{yr}^{-1}$. Note the similarity of the curves to the model argon supersaturation at this depth (dotted line). 
there may be no solution that exactly satisfies all the constraints. We chose the solution that best fit the data in a least-squares sense by using the method of singular value decomposition (Press et al., 1986).

First, the relevant indices were extracted from the observations (see Table $3-1$ ). The temperature record (Figure 3-6) yielded the mixed layer temperature difference between winter and summer. To determine the winter and summer values of argon supersaturation, the observed mixed layer averages were fit using a non-linear least squares technique to a functional form 4 that approximated the model curves (Figure 3-2a). Although we have used NBS-traceable argon standards and the best available values for argon solubility, a systematic offset of 0.2 $0.3 \%$ in the argon supersaturations is possible. Since the effects of a $0.3 \%$ bias in $\Delta \mathrm{Ar}$ are comparable to the formal uncertainties derived from the inversion, we do not consider this an important source of error. If an additional constraint is added to insure that all the solution values are non-negative, the results are almost identical except that the trapping mode fraction $f_{t}$ is forced to zero.

The helium supersaturations were fit by non-linear least squares to sine curves, which approximated the model results (Figure $3-2 \mathrm{~b}$ ). A systematic offset of up to $0.5 \%$ in the helium supersaturations is possible (Weiss, 1971b), but again the effects are comparable to the formal uncertainties derived from the inversion. We estimate an upper limit to these systematic effects of $\pm 15 \%$ for the gas exchange rate and $\pm 15 \%$ for the air injection amplitude. The maximum argon supersaturation in the summer thermocline was derived from Figure 3-1c. The oxygen data in Figure 3-1d were used with the error estimates derived earlier.

Each equation was weighted by the reciprocal of its estimated uncertainty, derived from estimated measurement errors (including uncertainty in the solubility) and model-linearization errors (Table 3-3). The solution and estimated uncertainties are compiled in Table 3-2. The residuals are within the estimated errors for each equation (see Table 3-3), suggesting that the solution is consistent with all constraint equations and that the estimated errors are reasonable.

The sensitivity of the solution to each linearized constraint equation is evident from the

${ }^{4}$ A skewed Gaussian form was used to fit the model curves:

$$
\Delta \operatorname{Ar}(t)=\Delta \operatorname{Ar}_{w}+\left(\Delta \operatorname{Ar}_{s}-\Delta \operatorname{Ar}_{w}\right) e^{\left(-[25+10(t-0.5)](t-0.5)^{2}\right)}
$$




\begin{tabular}{lcccccc}
\hline Property & & 1985 & 1986 & 1987 & $1985-1987$ avg. & $1960-1970$ avg. \\
\hline$\Delta \mathrm{T}_{m l}[s-w]$ & $\left({ }^{\circ} \mathrm{C}\right)$ & $9.0 \pm 0.2$ & $8.3 \pm 0.2$ & $8.3 \pm 0.2$ & $8.5 \pm 0.1$ & $8.2 \pm 0.2$ \\
$\Delta \mathrm{Ar}_{m l}[w]$ & $(\%)$ & $0.4 \pm 0.1$ & $0.5 \pm 0.1$ & $0.2 \pm 0.1$ & $0.4 \pm 0.1$ & $(0.4 \pm 0.1)$ \\
$\Delta \mathrm{Ar}_{m l}[s]$ & $(\%)$ & $3.3 \pm 0.3$ & $1.9 \pm 0.1$ & $2.7 \pm 0.1$ & $2.4 \pm 0.1$ & $(2.4 \pm 0.1)$ \\
$\Delta \mathrm{He}_{m l}[$ mean $]$ & $(\%)$ & $3.7 \pm 0.1$ & $3.3 \pm 0.1$ & $3.5 \pm 1.0$ & $3.5 \pm 0.1$ & $(3.5 \pm 0.1)$ \\
$\Delta \mathrm{He}_{\operatorname{ml}}[$ ampl $]$ & $(\%)$ & $0.5 \pm 0.1$ & $0.4 \pm 0.2$ & $0.4 \pm 0.4$ & $0.5 \pm 0.1$ & $(0.5 \pm 0.1)$ \\
$\Delta \mathrm{He}_{\operatorname{ml}}[w] / \Delta \mathrm{He}_{\operatorname{ml}}[s]$ & & $1.1 \pm 0.1$ & $1.3 \pm 0.1$ & $1.3 \pm 0.3$ & $1.2 \pm 0.1$ & $(1.2 \pm 0.1)$ \\
$\Delta \mathrm{Ar}_{\max }[s]$ & $(\%)$ & $4.0 \pm 0.3$ & $3.6 \pm 0.3$ & $4.0 \pm 0.3$ & $3.9 \pm 0.2$ & $(3.9 \pm 0.2)$ \\
$\Delta \mathrm{O}_{2 \operatorname{ml}}[s-w]$ & $(\%)$ & $17 \pm 3$ & $12 \pm 3$ & $9 \pm 3$ & $12.7 \pm 1.7$ & $10.8 \pm 0.6$ \\
$\Delta \mathrm{O}_{2 \max }[s-w]$ & $(\%)$ & $14 \pm 3$ & $14 \pm 3$ & $14 \pm 3$ & $14.0 \pm 1.7$ & $11.7 \pm 0.7$ \\
\hline
\end{tabular}

Table 3-1: Observed indices for 1985-1987. The temperature and oxygen indices for 1960-1970 were obtained from the historical record; the noble gases values in parentheses are approximated by the weighted averages of the 1985-1987 data.

\begin{tabular}{|c|c|c|c|c|c|}
\hline Solution Parameter & 1985 & 1986 & 1987 & $\begin{array}{c}1985-1987 \\
\text { average }\end{array}$ & $\begin{array}{l}1960-1970 \\
\text { average }\end{array}$ \\
\hline Vertical diffusivity $\left(10^{-4} \mathrm{~m}^{2} \mathrm{~s}^{-1}\right)$ & $0.91 \pm 0.08$ & $1.19 \pm 0.14$ & $1.20 \pm 0.14$ & $1.02 \pm 0.06$ & $1.26 \pm 0.16$ \\
\hline $\begin{array}{l}\text { Air injection } \\
\text { Amplitude (non-dim.) } \\
\text { Wind-speed exponent } \\
\text { Fraction "complete" trapping }\end{array}$ & $\begin{array}{r}0.4 \pm 0.1 \\
2.1 \pm 0.2 \\
-0.4 \pm 0.2\end{array}$ & $\begin{array}{r}0.5 \pm 0.1 \\
2.1 \pm 0.2 \\
-0.6 \pm 0.2\end{array}$ & $\begin{array}{r}0.3 \pm 0.2 \\
2.1 \pm 0.3 \\
-0.3 \pm 0.4\end{array}$ & $\begin{aligned} 0.4 & \pm 0.1 \\
2.1 & \pm 0.1 \\
-0.5 & \pm 0.1\end{aligned}$ & $\begin{array}{l}- \\
- \\
-\end{array}$ \\
\hline $\begin{array}{l}\text { Gas exchange rate } \\
\text { (rel. to Liss \& Merlivat curve) }\end{array}$ & $0.91 \pm 0.14$ & $1.58 \pm 0.60$ & $0.93 \pm 0.19$ & $0.94 \pm 0.11$ & - \\
\hline $\begin{array}{l}\text { New Production of } \mathrm{O}_{2} \\
\left(\text { moles } \mathrm{m}^{-2} \mathrm{yr}^{-1}\right) \\
\text { In mixed-layer } \\
\text { In seasonal thermocline }\end{array}$ & $\begin{array}{l}14.0 \pm 6.5 \\
4.2 \pm 1.7\end{array}$ & $\begin{array}{l}15.0 \pm 6.4 \\
5.1 \pm 1.7\end{array}$ & $\begin{array}{l}2.7 \pm 5.6 \\
5.7 \pm 1.7\end{array}$ & $\begin{array}{l}9.8 \pm 3.5 \\
5.0 \pm 1.0\end{array}$ & $\begin{array}{l}5.6 \pm 3.8 \\
4.3 \pm 0.5\end{array}$ \\
\hline $\begin{array}{l}\text { If mixed-layer production } \\
\text { fixed at zero }\end{array}$ & $5.8 \pm 1.5$ & $6.7 \pm 1.5$ & $6.1 \pm 1.5$ & $6.2 \pm 0.9$ & $4.6 \pm 0.4$ \\
\hline
\end{tabular}

Table 3-2: Inversion results. The 1985-1987 average represents the weighted average of the solutions for the individual years (similar results are obtained by a single inversion of the weighted average of the 1985-1987 data). The results for 1960-1970 were calculated by combining the temperature and oxygen data from this period with a weighted average of the 1985-1987 noble gas data (see Table 3-1). 
weighted matrix of equation coefficients (Table 3-4). For a given solution parameter, the magnitudes of the coefficients in that column reflect the relative importance of each of the constraint equations in determining the value of that parameter. The diffusivity is mainly determined from the mixed layer temperature history, and secondarily by the constraints from the subsurface argon cycle. Air injection amplitude is mainly determined by the mean helium supersaturation, as well as from the mixed layer argon supersaturation. The wind speed dependence of air injection is determined solely by the ratio of winter to summer helium supersaturations. The mixture of partial and total bubble trapping is determined by the relative mixed layer supersaturations of argon and helium, since the total air injection rate is normalized to the latter. The gas exchange rate is determined by the helium and argon supersaturations in the mixed layer. Surface productivity is barely constrained at all, as revealed by the small coefficient terms in this column. The subsurface productivity is determined by the subsurface oxygen data.

\subsection{Discussion}

\subsubsection{Vertical mixing}

The diffusivity that best matches the observed argon cycle $\left(1.0 \pm 0.1 \times 10^{-4} \mathrm{~m}^{2} \mathrm{~s}^{-1}\right)$ is consistent with that required to match the observed 1985-1987 temperature history and similar to the optimal value demanded by the heat budget for the climatological model runs (see Musgrave et al., 1988). This estimate is much larger than typical estimates of diapycnal mixing in the main thermocline of order $0.1 \times 10^{-4} \mathrm{~m}^{2} \mathrm{~s}^{-1}$ (Gargett, 1984; Jenkins, 1980). However, within the seasonal thermocline processes other than simple diapycnal diffusion may contribute to the apparent diffusivity. For example, Musgrave et al. (1988) have shown that wind-mixing is a significant component (about 25\%).

Musgrave et al. (1988) have demonstrated that the apparent vertical diffusivity required by the temperature cycle depends somewhat on the choice of light attenuation curve. Observations of light attenuation at Station S are scarce, but they suggest that it is bounded by Jerlov types I and IA. Musgrave et al. (1988) chose to use a type I curve based on the mapping of water types presented by Simonot \& Le Treut (1986), in which Station S appears as a singular point of type I water surrounded by type IA waters. We choose to use a type IA curve since this is 


\begin{tabular}{llllll}
\hline $\begin{array}{l}\text { Constraint } \\
\text { Equation }\end{array}$ & & $\begin{array}{l}\text { Linearization } \\
\text { Error }\end{array}$ & $\begin{array}{l}\text { Measurement } \\
\text { Error }\end{array}$ & $\begin{array}{l}\text { Total } \\
\text { Error }\end{array}$ & $\begin{array}{l}\text { Inversion } \\
\text { Residual }\end{array}$ \\
\hline$(3.1) \Delta \mathrm{T}_{m l}[s-w]$ & $\left({ }^{\circ} \mathrm{C}\right)$ & 0.2 & 0.2 & 0.3 & 0.0 \\
$(3.2) \Delta \mathrm{Ar}_{\text {max }}[s]$ & $(\%)$ & 0.1 & 0.4 & 0.4 & -0.1 \\
$(3.3) \Delta \mathrm{Ar}_{\text {ml }}[s]$ & $(\%)$ & 0.1 & 0.4 & 0.4 & +0.1 \\
$(3.4) \Delta \mathrm{Ar}_{\text {ml }}[w]$ & $(\%)$ & 0.1 & 0.3 & 0.3 & 0.0 \\
$(3.5) \Delta \mathrm{He}_{m l}[$ mean $]$ & $(\%)$ & 0.1 & 0.2 & 0.2 & 0.0 \\
$(3.6) \Delta \mathrm{He}_{\text {ml }}[w] / \Delta \mathrm{He}_{\text {ml }}[s]$ & & 0.2 & 0.1 & 0.2 & 0.0 \\
$(3.7) \Delta \mathrm{O}_{2 \text { ml }}[s-w]$ & $(\%)$ & 0.5 & 3.0 & 3.0 & 0.0 \\
$(3.8) \Delta \mathrm{O}_{2 \text { max }}[s-w]$ & $(\%)$ & 0.2 & 3.0 & 3.0 & 0.0 \\
\hline
\end{tabular}

Table 3-3: For each constraint equation, there is an error associated with linearizing the model response (first column), and an uncertainty associated with measuring features of the tracer distributions (second column), including analytical errors and uncertainty in the solubilities. In most cases, the linearization error is smaller than the measurement error. The total uncertainty for each constraint equation (third column) is calculated as the quadrature sum of the linearization and measurement errors. The final column shows the residuals obtained by comparing the results of the inverse calculation to the data (1985 in this case). For each equation, the inversion residual is smaller than the total error associated with that constraint, suggesting that the solution (for 1985) is consistent with all the constraint equations. Results for 1986 and 1987 are similar.

\begin{tabular}{lrrrrrrr}
\hline & \multicolumn{7}{c}{ Model Parameter } \\
Constraint Eqn. & $1 / \kappa$ & $a_{\text {inj }}$ & $\gamma$ & $f_{t}$ & $1 / g$ & $P_{m l}$ & $P_{50}$ \\
\hline$(3.1) \Delta \mathrm{T}_{m l}[s-w]$ & 9.9 & 0 & 0 & 0 & 0 & 0 & 0 \\
$(3.2) \Delta \operatorname{Ar}_{\text {max }}[s]$ & 2.0 & 4.4 & 0 & 0.8 & 5.0 & 0 & 0 \\
$(3.3) \Delta \operatorname{Ar}_{m l}[s]$ & 0 & 6.7 & 0 & 0.8 & 8.0 & 0 & 0 \\
$(3.4) \Delta \operatorname{Ar}_{m l}[w]$ & 0 & 9.0 & 0 & 4.0 & 3.0 & 0 & 0 \\
$(3.5) \Delta \mathrm{He}_{m l}[m e a n]$ & 0 & 23 & 0 & 0 & 11 & 0 & 0 \\
$(3.6) \Delta \mathrm{He}_{m l}[w] / \Delta \mathrm{He}_{m l}[s]$ & 0 & 0 & 5.7 & 0 & 0 & 0 & 0 \\
$(3.7) \Delta \mathrm{O}_{2 m l}[s-w]$ & 0 & -0.3 & 0 & 0 & 5.7 & 0.2 & 0.1 \\
$(3.8) \Delta \mathrm{O}_{2 m a x}[s-w]$ & 1.3 & -0.2 & 0 & 0 & 0.7 & 0.06 & 0.7 \\
\hline
\end{tabular}

Table 3-4: The linearized model constraints form an overdetermined set of simultaneous linear equations. Each equation is weighted by the reciprocal of the total error associated with that equation (see Table 3-3). The weighted matrix of coefficients in these equations allows a direct comparison of the relative importance of each constraint in determining the solution (see text for details). 
more typical of the waters advected by Station S by the mean gyre circulation. Our choice of type IA increases our estimate of apparent vertical diffusivity by about $25 \%$ and increases the required oxygen production by approximately $10 \%$ relative to their estimate. Although there are also some uncertainties in the estimates for heat flux and Ekman pumping, these values are well-constrained by the seasonal evolution of temperature, and contribute an uncertainty of order $20 \%$ to the required oxygen production (Musgrave et al., 1988).

\subsubsection{Air injection}

Our estimate of air injection indicates that this process contributes about $25 \%$ of the argon supersaturation observed in the summer mixed layer, a somewhat larger effect than predicted by Atkinson (1973). Air injection probably accounts for about this fraction of the ubiquitous $3 \%$ oxygen supersaturation noted by Broecker \& Peng (1982) in the GEOSECS surface water data; the balance is likely due to radiative heating and biological production. Our estimates of the rate of bubble trapping are also of the same order of magnitude as those by Thorpe (1984; 1986) based on single-bubble dynamics applied to bubble populations measured by acoustic scattering. The wind-speed dependence of this rate is similar to the wind-speed dependence of bubble populations estimated by Crawford and Farmer (1987; $\gamma=3.0 \pm 0.5$ ) from open ocean acoustic measurements. By combining their estimates of bubble concentrations and size distribution with our estimate of the bubble flux, we estimate a mean bubble lifetime of order 10 seconds. This calculation suggests that the bubble dynamics may be complex, since this lifetime is of the same order as the transit time to the surface, the time for bubble dissolution, and the time-scale for gas exchange between the bubble and bulk seawater (Jahne et al; 1984). Moreover, this rough agreement is consistent with a predominance of the partial bubble trapping process, as inferred from the helium and argon supersaturations. By applying the model described here to GEOSECS measurements of $\triangle \mathrm{He}$ and $\triangle \mathrm{Ne}$, Jenkins (1988b) also found that air injection was dominated by partial trapping rather than complete trapping, although the contribution of total trapping appeared to be more significant. 


\subsubsection{Gas exchange}

The gas exchange rates during 1985-1987 are slightly lower than our best estimate using the Liss \& Merlivat (1986) formulation of piston velocity and climatological wind speeds. The agreement is remarkable, considering that the field data are mostly from ${ }^{222} \mathrm{Rn}\left(\approx 5\right.$ day timescale) and ${ }^{14} \mathrm{C}$ ( $\approx 2$ year timescale) estimates whereas the argon cycle gives a seasonal average. The discrepancy is comparable to the uncertainty in the wind speeds $( \pm 10 \%)$ and in the piston velocity vs. wind speed relationship ( $\pm 15 \%)$ (Jenkins, 1988b).

The supersaturations observed for helium and argon imply that gas exchange is removing these gases from the mixed layer year-round. Air injection balances these losses by "pumping" gas back in. For a typical model run, the balance between gas exchange and air injection is indicated in Figure 3-10 for helium and argon. For helium, the close balance between these two processes accounts for nearly constant supersaturations. For argon, air injection exceeds outgassing during the wintertime but offsets only $25 \%$ of the outgassing rate in summer. The $0.4 \%$ increase in barometric pressure between winter and summer also contributes to the ingassing rate by increasing the effective solubility, and helps to close the annual cycles. If a constant barometric pressure of 1 atmosphere is used in the model, the required gas exchange rate decreases by about $15 \%$.

The interaction between these processes can affect measurements of apparent gas exchange rates since gas exchange is a two-way process always acting to restore solubility equilibrium, whereas air injection always tends to increase mixed layer concentrations. In the data we have obtained, gas exchange and air injection work in opposite directions to determine the mixed layer gas balances. In field measurements of outgassing by the ${ }^{222} \mathrm{Rn}$ method, air injection will have little effect on the apparent gas exchange rate (since radon is relatively insensitive to air injection due its high solubility). In wind tunnel ingassing experiments, air injection will tend to enhance the apparent gas exchange rate. This may help to explain why laboratory rates are typically higher than field measurements (Figure 1-5).

Short period variations in wind speed tend to increase the mixed layer supersaturations of helium and argon because the air injection rate has a strongly non-linear dependence on wind speed. Although the Liss \& Merlivat (1986) parametrization of gas exchange rate also has a non-linear dependence on wind speed, this introduces only a small bias $(<5 \%)$ in the seasonally 
(a)

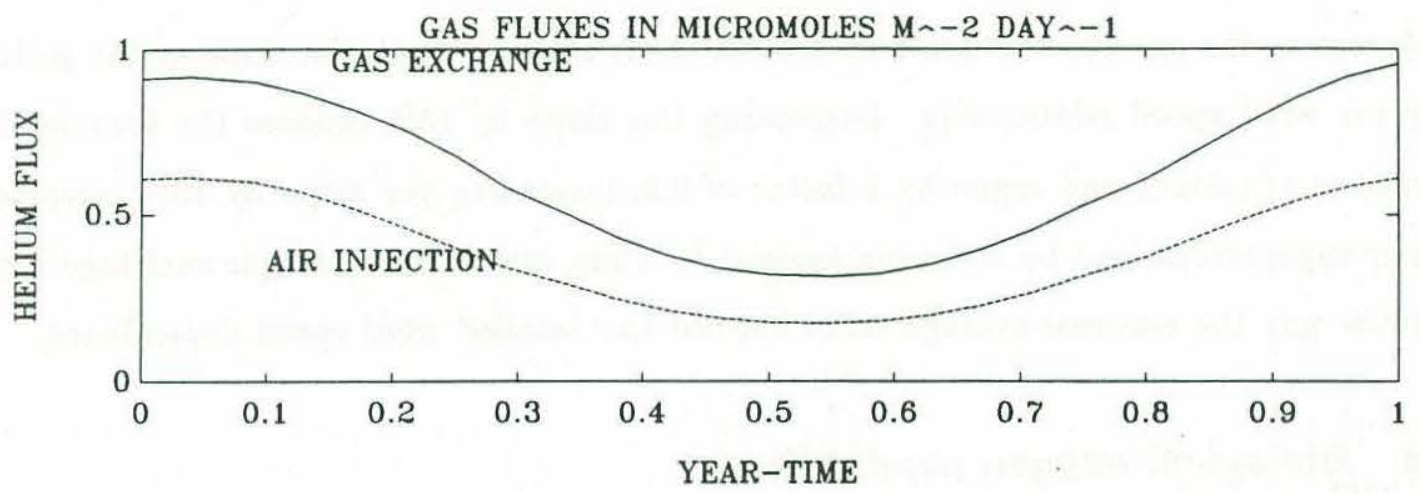

(b)

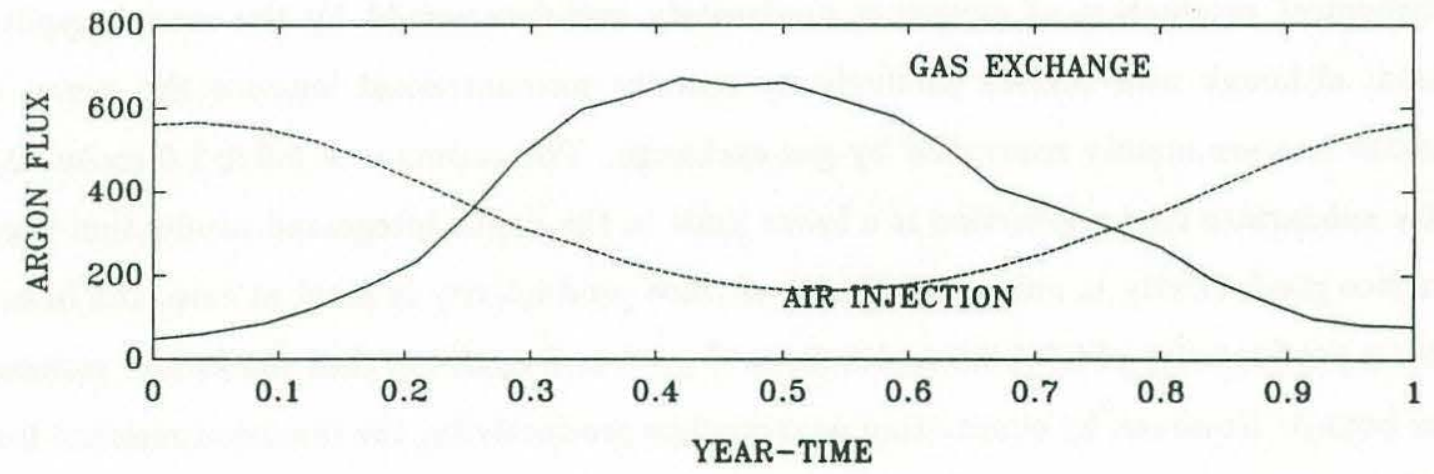

Figure 3-10: Model gas exchange (solid lines) and air injection (dashed lines) fluxes for (a) helium and (b) argon for a typical model run, in units of $\mu$ moles $\mathrm{m}^{-2}$ day ${ }^{-1}$. The $0.4 \%$ increase in barometric pressure between winter and summer adds to the effective ingassing rate for each gas, resulting in closed annual cycles. 
averaged gas exchange rate because the changes in slope are relatively small. The wind speed variance in our simulation is within $25 \%$ of that obtained by Musgrave et al. (1988), who used stochastic winds based on the wind spectrum measured by a meteorological buoy west of Bermuda. The net effect of doubling the amplitude of the short period wind variations is to increase the supersaturations by a factor of 1.1-1.2; halving the amplitude decreases the supersaturations by a factor of 0.90-0.95.

Moreover, the gas supersaturations are relatively insensitive to the slope of the piston velocity vs. wind speed relationship. Decreasing this slope by $15 \%$ reduces the summer supersaturations of helium and argon by a factor of 0.9 ; increasing the slope by $15 \%$ increases the summer supersaturations by a similar amount ${ }^{5}$. Thus, our estimate of gas exchange rate can constrain only the seasonal-average value but not the detailed wind-speed dependence.

\subsubsection{Biological oxygen production}

The biological production of oxygen is moderately well-determined by the model applied to the data, although near-surface productivity remains unconstrained because the mixed layer concentrations are mainly controlled by gas exchange. The estimate of $5.0 \pm 1.0$ moles $\mathrm{O}_{2} \mathrm{~m}^{2}$ $\mathrm{yr}^{-1}$ for subsurface new production is a lower limit to the depth-integrated production because the surface productivity is unknown. If near-surface productivity is fixed at zero, the inversion predicts a productivity of $6.2 \pm 0.9$ moles $\mathrm{O}_{2} \mathrm{~m}^{-2} \mathrm{yr}^{-1}$ and confirms that the former estimate is a lower bound. However, by eliminating near-surface productivity, the inversion residual for the mixed layer oxygen constraint is increased (the model predicts supersaturations smaller than observed). Nevertheless, the latter estimate may be closer to the depth-integrated value since unconstrained near-surface production tends to reduce the required subsurface production in the former estimate. We would expect that near-surface new production is minimal on the basis of ${ }^{15} \mathrm{~N}$-uptake experiments (e.g. Lewis et al., 1986) which suggest that near-surface production is supported mainly by recycled nutrients (e.g. $\mathrm{NH}_{3}$ ) rather than "new" nutrients (e.g. NO3).

Although we do not know the true depth dependence of new production, model runs suggest that the depth of the observed maximum in supersaturation corresponds to the depth of

\footnotetext{
${ }^{5}$ The Liss \& Merlivat (1986) curve was modified for this experiment by altering the slope for wind speeds in the range $3.6-13 \mathrm{~m} \mathrm{~s}^{-1}$ while keeping a constant value at $8.5 \mathrm{~m} \mathrm{~s}^{-1}$ (the annual mean wind speed).
} 
maximum production, as long as the subsurface production exceeds that in the mixed layer. Since the data show a supersaturation maximum at about 50 meters, we have some justification for choosing a production curve with a corresponding maximum.

Our production estimate will tend to be conservative because the production required to produce the observed supersaturation $\left(\Delta \mathrm{O}_{2} \max [s-w]\right.$ in excess of the supersaturation generated by physical effects) is a minimum at 50 meters (Figure 3-11). If the depth of maximum production is raised to 25 meters, the required production increases by $30 \%$; if it is lowered to 75 meters, the production required increases by $6 \%$. The asymmetry occurs because oxygen losses via mixing and gas exchange increase rapidly toward the surface, increasing the production required to produce supersaturations. Deeper in the seasonal thermocline, it becomes harder to produce supersaturations due to oxygen consumption below the euphotic zone.

We have estimated the seasonal modulation in oxygen production from the flux of organic matter to a deep sediment trap at a nearby site (Deuser, 1986), but some uncertainty uncertainty remains regarding the timing of new production. As shown in Figure 3-12, the production required to produce the observed supersaturation at 50 meters $\left(\Delta \mathrm{O}_{2} \max [s-w]\right.$ in excess of that due to physical effects) is at a minimum between spring and summer, since the stratification inhibits transfer of oxygen to the mixed layer where it is subsequently lost through gas exchange. During fall and winter, high gas exchange rates in the absence of stratification lead to large losses. In late winter, deep convection increases the time constant for mixed layer gas exchange enough to allow oxygen to accumulate. Altering the timing of production could lower our estimate by a factor of 0.5 or raise it by as much as a factor of 5 ; thus, our estimate is near the minimum value. Although the required production is sensitive to the timing of a short pulse of production (e.g. a phytoplankton bloom), model runs indicate that the width of the pulse is relatively unimportant since the oxygen accumulation in the seasonal thermocline integrates over timescales of several weeks.

For the 1985-1987 record, our determination of subsurface production may be limited by the quality of the oxygen data. If the analytical error in the oxygen data were $\pm 0.05 \mathrm{ml} \mathrm{1}^{-1}$ subsurface production could be constrained within $\pm 10 \%$. In fact, the $1960-1970$ average data are known at this level of precision (see Table 3-1). If the oxygen and temperature data from this period are combined with the averaged noble gas data from 1985-1987, the inverse calculation 


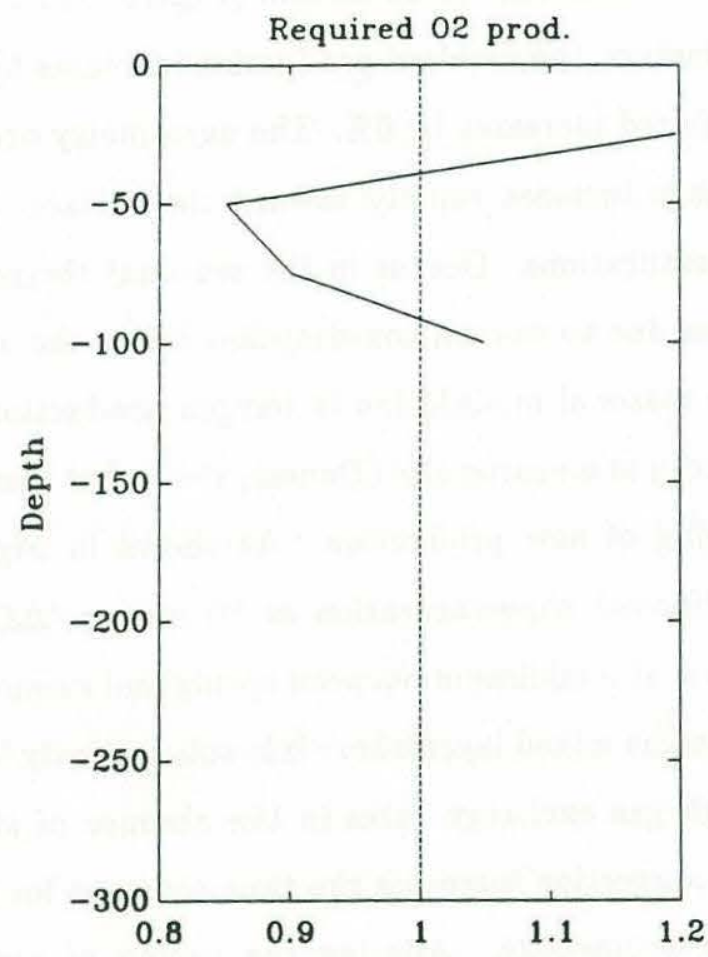

Figure 3-11: Required oxygen production vs. depth of maximum production. 


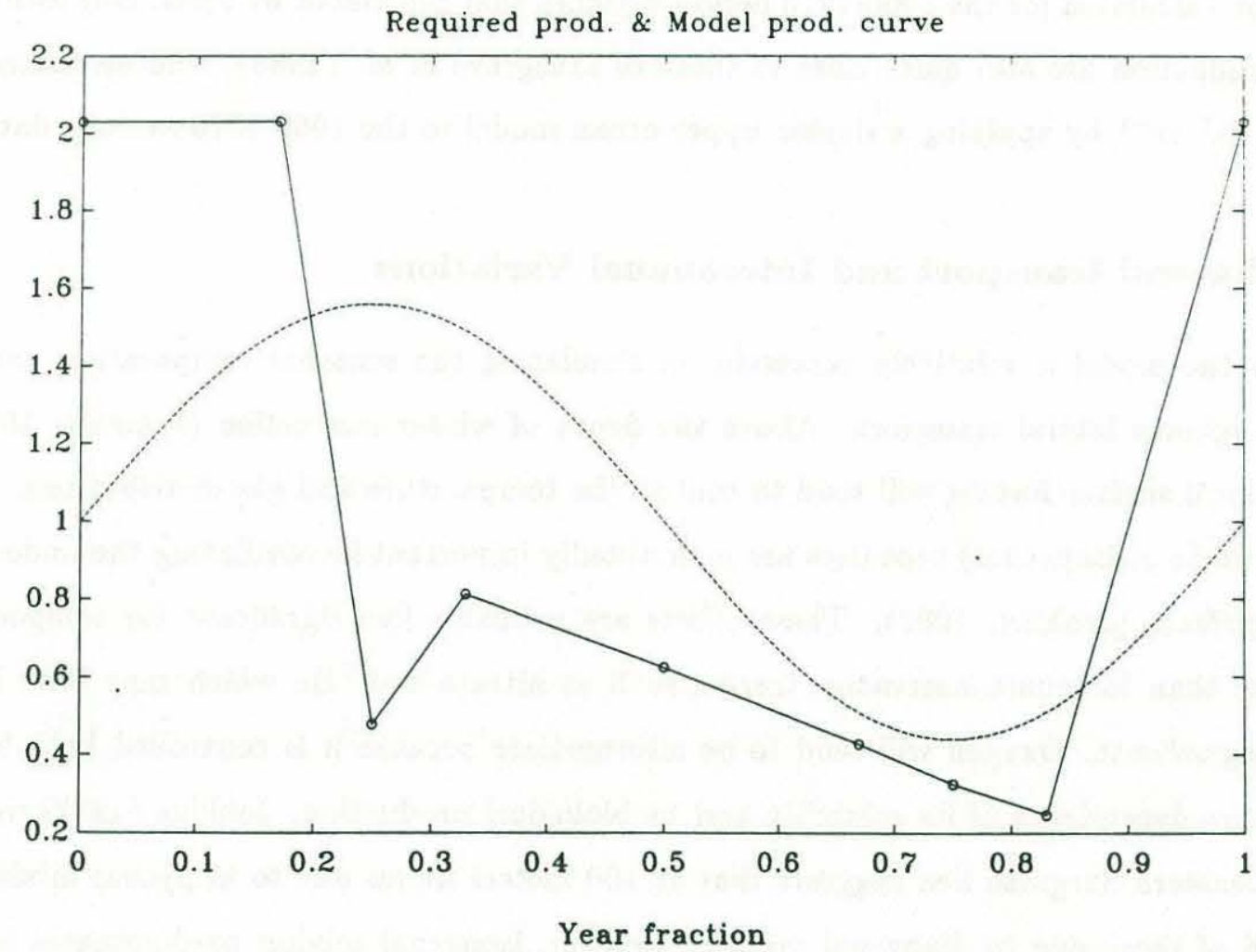

Figure 3-12: $\quad$ Required oxygen production vs. time of production (solid line), and the seasonal modulation of oxygen production used in the model (dashed line). 
yields values for $\kappa, P_{m l}$, and $P_{50}$ as shown in Table 3-2. Clearly, the two data sets are not strictly comparable; however, this calculation suggests that new production during 1960-1970 was similar to that in 1985-1987 if gas exchange rates for both periods were comparable. In any case, the comparison indicates that better oxygen measurements would constrain the subsurface productivity more tightly, although near-surface productivity would remain unconstrained. New production calculated for the 1960-1970 period matches that calculated by J\&G. Our estimates of new production are also quite close to those of Musgrave et al. (1988), who estimated 3-4 moles $\mathrm{O}_{2} \mathrm{~m}^{2} \mathrm{yr}^{-1}$ by applying a similar upper ocean model to the 1960-1970 average data.

\subsubsection{Lateral transport and Interannual Variations}

Although the model is relatively successful in simulating the seasonal temperature and gas cycles, it ignores lateral transport. Above the depth of winter convection (typically 150-200 meters), local surface forcing will tend to control the temperature and gas distributions. However, lateral (e.g. isopycnal) processes are undoubtedly important in ventilating the underlying density surfaces (Jenkins, 1980). These effects are probably less significant for temperature and argon than for non-conservative tracers such as nitrate and ${ }^{3} \mathrm{He}$ which may have larger isopycnal gradients. Oxygen will tend to be intermediate because it is controlled both by the temperature dependence of its solubility and by biological production. Jenkins (1987) work in the southeastern Sargasso Sea suggests that at 100 meters fluxes due to isopycnal mixing are about $1 \%$ of those due to diapycnal mixing; however, isopycnal mixing predominates by 200 meters. Isopycnal mixing will tend to reduce the size of subsurface anomalies. Since we have not accounted for isopycnal mixing, we may be underestimating the magnitude of the oxygen excess produced in the seasonal thermocline. Thus, we may be underestimating the biological oxygen production required.

The importance of processes other than vertical mixing becomes evident when the model attempts to simulate the distributions of nitrate and ${ }^{3} \mathrm{He}$. In order to balance losses near the surface (outgassing of ${ }^{3} \mathrm{He}$ and biological uptake of nitrate), a vertical diffusivity of order $10^{-3}$ $\mathrm{m}^{2} \mathrm{~s}^{-1}$ is required to close the annual cycle. This is an order of magnitude higher than that demanded by the temperature and argon distributions and unreasonably large by any standard. If we use this value in simulating the oxygen cycle, the model is unable to simulate a subsurface 
maximum in oxygen supersaturation since gas exchange becomes the rate-limiting process for oxygen loss from the seasonal thermocline.

In fact, lateral effects may explain some of the differences between the 1985-1987 data and the composite seasonal cycle discussed by J\&G. Although the basic features are similar, the subsurface oxygen maximum in 1985 is substantially larger than in the average record and persists well into the fall, during which the upper 200 meters were also much warmer than average. Furthermore, the hydrographic structure observed in July, 1986 (Station 593) suggests the presence of an anticyclonic eddy (see Chapter 4 for details). Although the oxygen and temperature cycles in 1985-1987 are well within the ranges observed for individual years in the historical record at Station S, the one-year records appear "noisier". This is not surprising because the longer record will tend to smooth out irregularities due to passage of advected features (e.g. mesoscale eddies) and interannual variations in meteorological forcing (e.g. wind stress, air-sea heat flux).

How representative is the $1985-1987$ period of climatological mean conditions? To answer this question, we need to know how the physical forcing varies with time. As mentioned earlier, the wind speeds during 1985-1987 appear to be representative of climatological mean values (Figure 3-3). Although we do not have a complete record for heat flux, we have made a crude estimate of changes in the heat content of the water column using the temperature record (Figure 3-14). Work by Talley \& Raymer (1982), Jenkins (1982), J\&G, and Druffel (1988) suggest that ventilation rates rose to a maximum in the mid-1960's, and then decreased until 1978 when a severe winter (Leetma, 1977; Worthington, 1977) restored conditions typical period prior to 1960. The changes in heat content and mixed layer depth (Figure 3-13) appear to be consistent with these estimates of variations in subtropical mode water formation and in water mass renewal rates. Well-ventilated periods are characterized by high water mass renewal rates, high heat flux, and deep winter mixed layers; poorly-ventilated periods exhibit sluggish water mass renewal rates, low heat flux, and shallow winter convection. The 1985-1987 period appears to be typical of climatological mean conditions.

Although there are some significant differences between the data from the three years in our study, they are not apparent in our estimates of physical transport and biological production. The winter mixed layer in 1985 was unusually deep and contained a large pool of nitrate. 


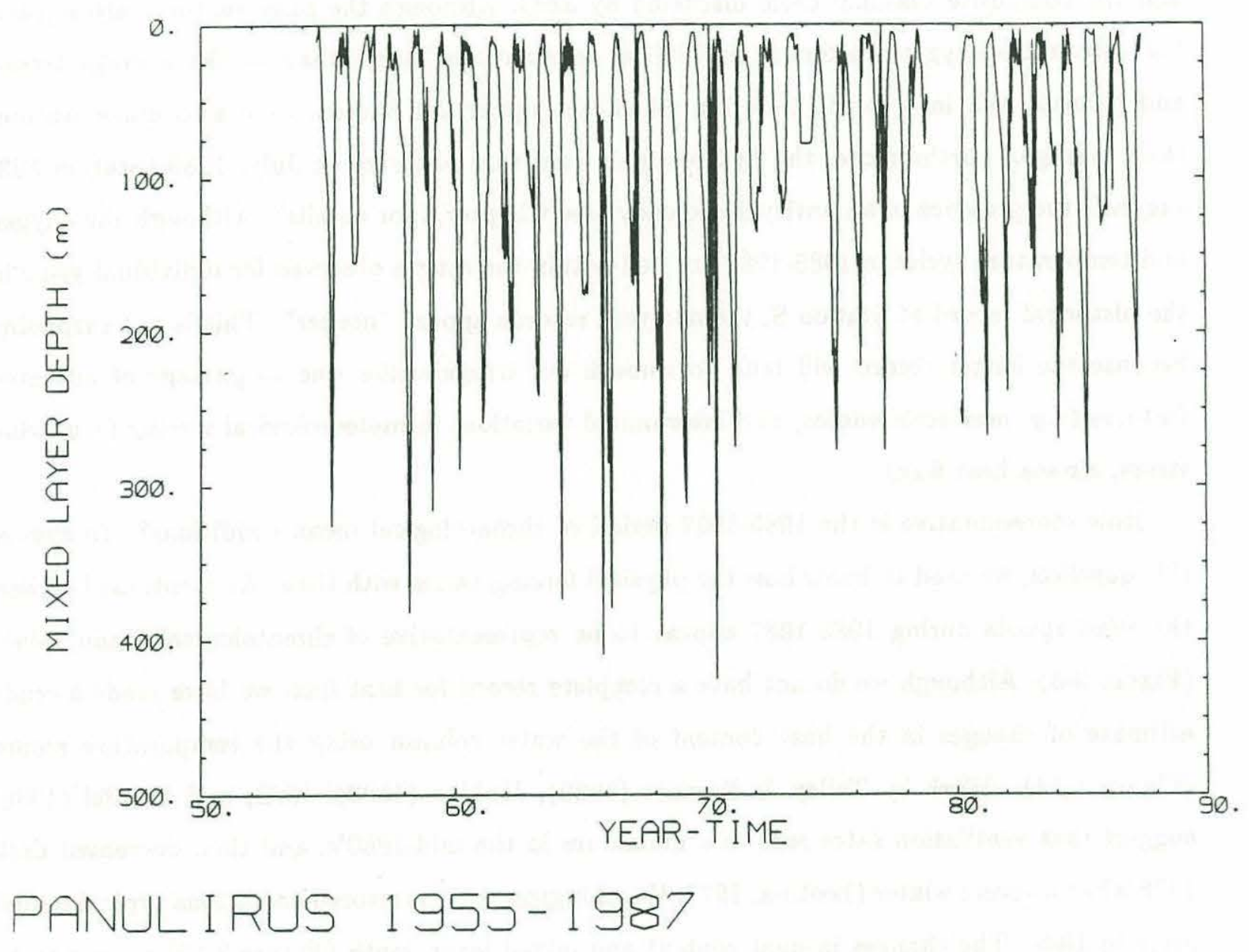

Figure 3-13: Mixed layer depths at Station $\mathrm{S}$, determined using a $\Delta \mathrm{T}=0.5^{\circ} \mathrm{C}$ criterion. 


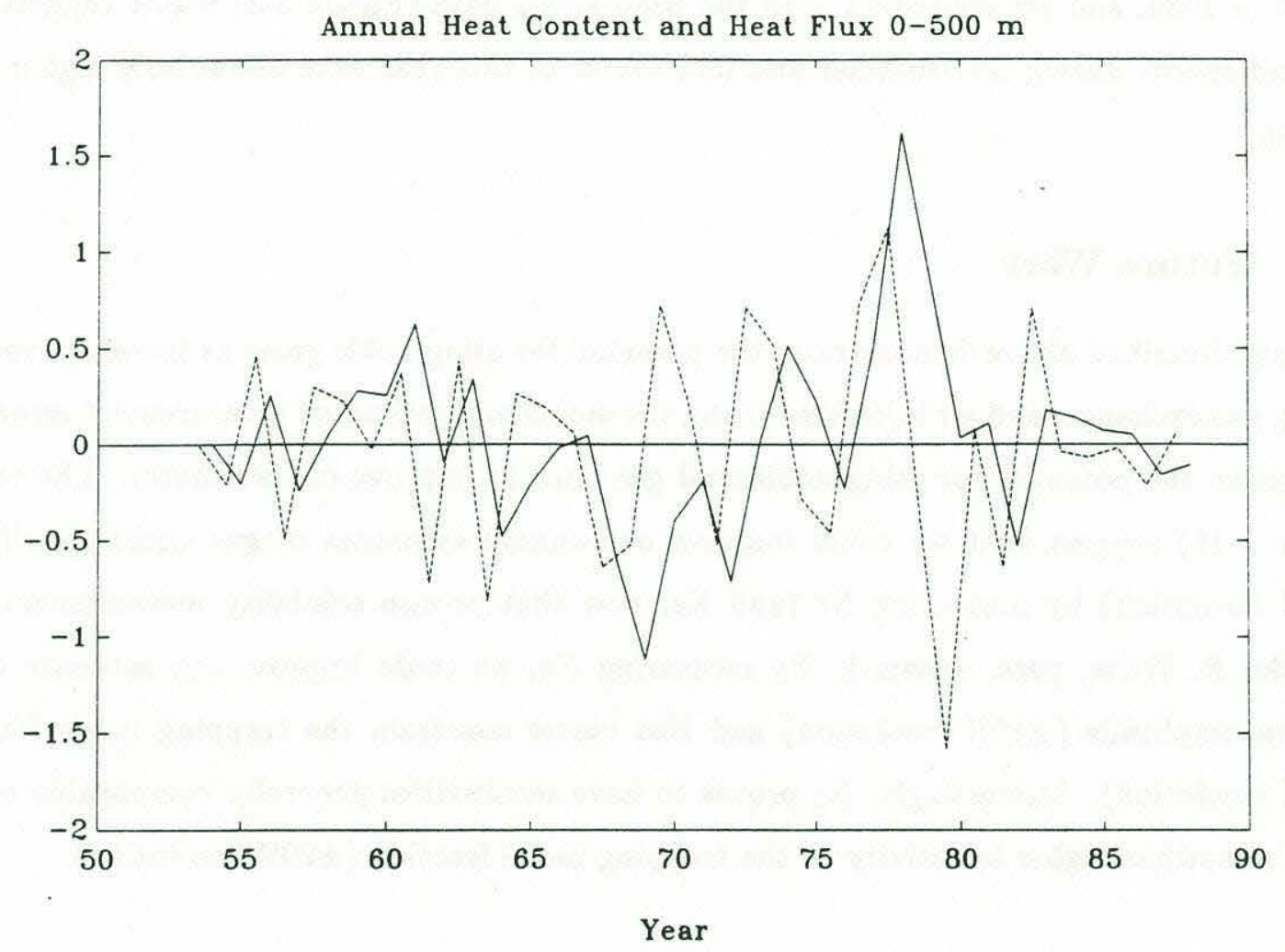

Figure 3-14: Heat content (solid line) of the upper 500 meters, expressed relative to the effective mean temperature of this layer. Heat flux (dashed line) is computed as the time derivative of the heat content; positive heat fluxes indicate heat transfer into the ocean. 
In 1986, winter convection was less vigorous and nitrate was absent, whereas 1987 was an intermediate case. Although one might expect a correlation between the availability of nutrients in the late winter and the amount of new production for the subsequent year, this is not borne out by the inversion results. Another process (e.g. nutrient injection by mesoscale eddies) may be providing much of the nutrients, especially in 1986. The estimated gas exchange rates appear highest in 1986, and are consistent with the wind speed data (Figure 3-3) which suggest that the wind speeds during mid-summer and mid-winter of this year were about $30 \%$ higher than average.

\subsubsection{Future Work}

The work described above demonstrates the potential for using noble gases as tracers of vertical mixing, gas exchange, and air injection. Using the model and estimated measurement errors, we can explore the potential for using additional gas data to improve our estimates. The results (Figure 3-15) suggest that we could improve our annual estimates of gas exchange slightly ( $\pm 10 \%$ resolution) by measuring $\mathrm{Kr}$ (and $\mathrm{Xe}$, now that precise solubility measurements are available; R. Weiss, pers. comm.). By measuring Ne, we could improve our estimate of air injection amplitude ( $\pm 10 \%$ resolution) and also better constrain the trapping mode fraction ( $\pm 45 \%$ resolution). Interestingly, $\mathrm{N}_{2}$ proves to have sensitivities generally comparable to $\mathrm{Ne}$, with a somewhat higher sensitivity to the trapping mode fraction ( $\pm 20 \%$ resolution).

\subsection{Summary}

The seasonal cycle of argon near Bermuda demonstrates unequivocally that only a small fraction of the subsurface oxygen supersaturation maximum can be attributed to physical causes, and therefore that most of the supersaturation must be attributed to photosynthetic oxygen production. Furthermore, the evolution of argon concentration in the seasonal thermocline suggests that most of the oxygen produced there is also lost due to vertical mixing and gas exchange. The subsurface evolution of argon concentration requires a vertical diffusivity in the seasonal thermocline of $1.0 \pm 0.1 \times 10^{-4} \mathrm{~m}^{2} \mathrm{~s}^{-1}$, consistent with that derived from temperature. Measurements of argon and helium supersaturation determine the rate of air injection to $\pm 15 \%$ 


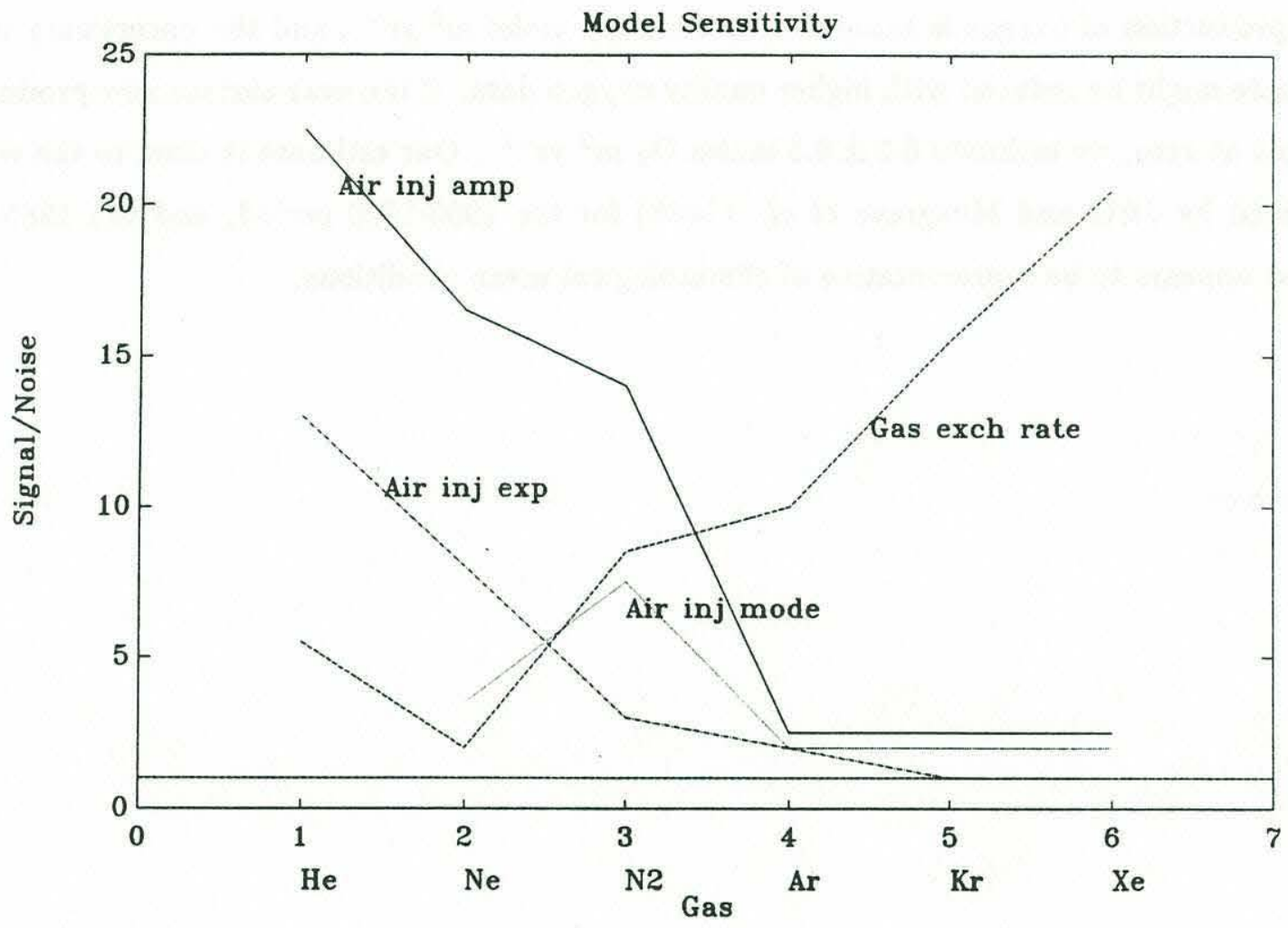

Figure 3-15: Sensitivity of the inversion to the noble gases and nitrogen, plotted as the effective signal/noise ratio provided by each tracer (e.g. a ratio of 10 indicates that the solution parameter could be determined by $\pm 10 \%)$. 
and the seasonally-averaged gas exchange rate to $\pm 11 \%$. The inferred rate of bubble trapping is roughly consistent with previous work by Thorpe $(1984 ; 1986)$, and Crawford and Farmer (1987), and this process is dominated by partial trapping as suggested by Jenkins (1988b). Our estimate of gas exchange rate is consistent within errors with that predicted from climatological wind speeds and Liss \& Merlivat's (1986) formulation of piston velocity. A lower limit to new production of oxygen is estimated as $5.0 \pm 1.0$ moles $\mathrm{m}^{2} \mathrm{yr}^{-1}$, and the uncertainty in the estimate might be reduced with higher quality oxygen data. If the near-surface new production is fixed at zero, we estimate $6.2 \pm 0.5$ moles $\mathrm{O}_{2} \mathrm{~m}^{2} \mathrm{yr}^{-1}$. Our estimate is close to the results obtained by J\&G and Musgrave et al. (1988) for the 1960-1970 period, and the 1985-1987 period appears to be representative of climatological mean conditions. 


\section{Chapter 4}

\section{Nutrient Injection into the}

\section{Euphotic Zone}

\subsection{Introduction}

Recently, Jenkins (1988a) estimated the annual flux of nitrate into the euphotic zone near Bermuda by calculating the upward flux of ${ }^{3} \mathrm{He}$ and applying an empirical ${ }^{3} \mathrm{He}$-nitrate correlation in the main thermocline. This nitrate flux $\left(0.6 \pm 0.2\right.$ moles $\left.\mathrm{m}^{-2} \mathrm{yr}^{-1}\right)$ is consistent with independent estimates of oxygen production in the euphotic zone and oxygen consumption in the main thermocline (J\&G; Spitzer \& Jenkins, 1989). However, the ${ }^{3} \mathrm{He}$ and nitrate fluxes would imply unreasonably large vertical diffusivities (order $10^{-3} \mathrm{~m}^{2} \mathrm{~s}^{-1}$; Jenkins, 1988a) if the vertical flux is maintained solely by turbulent mixing. ${ }^{1}$.

Jenkins (1988b) has observed an apparent tracer "injection" event that may explain a significant portion of these fluxes. We propose that the vertical structure associated with this event indicates the presence of an anticyclonic mesoscale eddy. We use the 35 year hydrographic record at Station $S$ to identify similar events in the past. By examining their timing and frequency, we estimate their significance for the ventilation of the subtropical gyre and the supply of nutrients to surface waters.

\footnotetext{
${ }^{1}$ Observed nitrate gradients at the base of the euphotic zone of 0.005-0.05 $\mu \mathrm{M} \mathrm{m}^{-1}$ would demand an apparent vertical diffusivity of $0.4-4 \times 10^{-3} \mathrm{~m}^{2} \mathrm{~s}^{-1}$. To simulate the seasonal thermal cycle at Station $\mathrm{S}$, a vertical diffusivity of $10^{-4} \mathrm{~m}^{2} \mathrm{~s}^{-1}$ is required (Spitzer \& Jenkins, 1989); estimates based on microstructure suggest turbulent diffusivities 2-10 times even lower (Lewis et al., 1986).
} 


\subsection{Observations}

The event, observed during an occupation of Station S in July, 1986 (station 593), was accompanied by significant amounts of nitrate $(>0.5 \mu \mathrm{M})$ and excess ${ }^{3} \mathrm{He}$ in the euphotic zone (Figure 1-1). The ${ }^{3} \mathrm{He}$ (and nitrate) must have come from below since only $10 \%$ of the excess could have accrued from in situ tritium decay. The depth-integrated excess ${ }^{3} \mathrm{He}$ and nitrate above 150 meters (the average depth of winter convection) suggests that this event could account for $5-15 \%$ of the annual flux of these tracers into the euphotic zone.

The temperature profile at this station ${ }^{2}$ is characterized by an elevation of the seasonal thermocline and depression of the main thermocline, with an unusually thick layer of subtropical mode water $\left(18^{\circ} \mathrm{C}\right.$ water) in between (Table 1-1). Brundage \& Dugan (1986) found a similar pattern of vertical displacements (Figure 1-3) in an anticyclonic mesoscale eddy observed in temperature (XBT) and current velocity (XCP) sections near $31^{\circ} \mathrm{N} 64^{\circ} \mathrm{W}$ during the summer of 1981. These authors also document numerous other occurrences of such eddies in the Sargasso Sea, and we suggest here that this type of eddy is responsible for the profiles at station 593 . The rotational speeds of these eddies (up to $50 \mathrm{~cm} \mathrm{~s}^{-1}$ ) are an order of magnitude larger than their translational speeds (4-5 $\mathrm{cm} \mathrm{s}^{-1}$ ), indicating that they are dynamical entities with energy comparable to Gulf Stream rings ${ }^{3}$. Their relationship to other types of isothermal lenses deeper in the thermocline or to other anticyclonic eddies is unknown. The details of their formation and decay are also uncertain.

The unusual thermal structure at station 593 is reflected in other tracer profiles. In the subtropical mode water, the apparent oxygen utilization ( $\mathrm{AOU}$ ), nitrate, and ${ }^{3} \mathrm{He}$ are at about half their climatological mean values (Table 1-1) indicating that this layer has been recently ventilated. However, the temperature of the thermostad is also somewhat warmer than the climatological mean value. The tracer values are within the range expected for this temperature (Figures 1-2 and 1-5), suggesting that typical tracer-temperature relationships hold within the upper layers of the eddy (above 500 meters).

\footnotetext{
${ }^{2}$ Expendable bathythermograph (XBT) drops before and after the bottle casts confirm the unusual thermal structure.

${ }^{3}$ However, note that they are quite distinct from Gulf Stream cold-core rings in the Sargasso Sea, where isotherm displacements are in the opposite sense.
} 

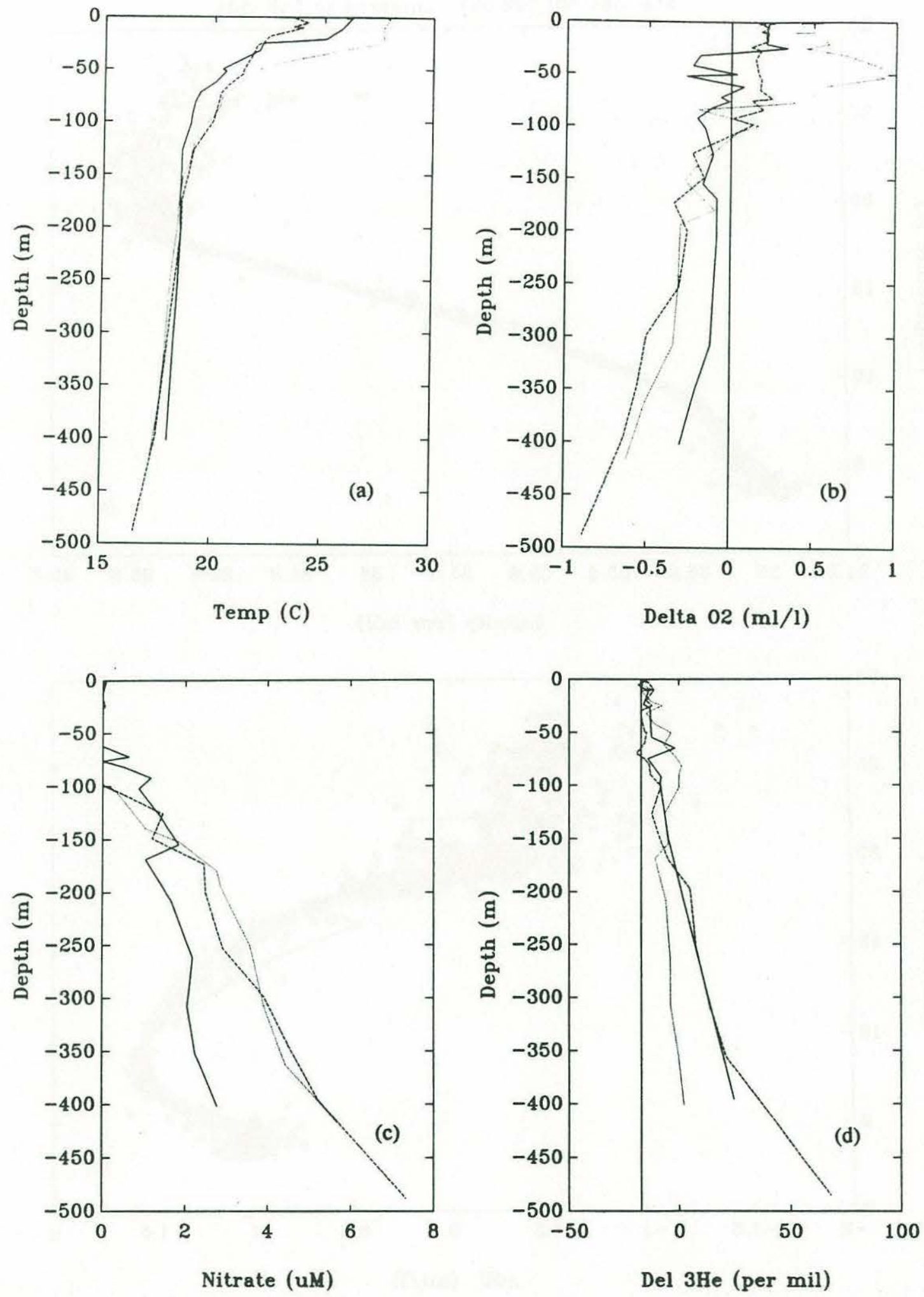

Figure 4-1: Profiles at station 593 for: (a) temperature, (b) $\Delta \mathrm{O}_{2}$ (or - $\mathrm{AOU}$ ), (c) $\delta^{3} \mathrm{He}$, (d) nitrate. for the anomalous station 593 (solid line), the previous station (dotted line), the following station (dashed line), and all the 1985-1987 data (points). 

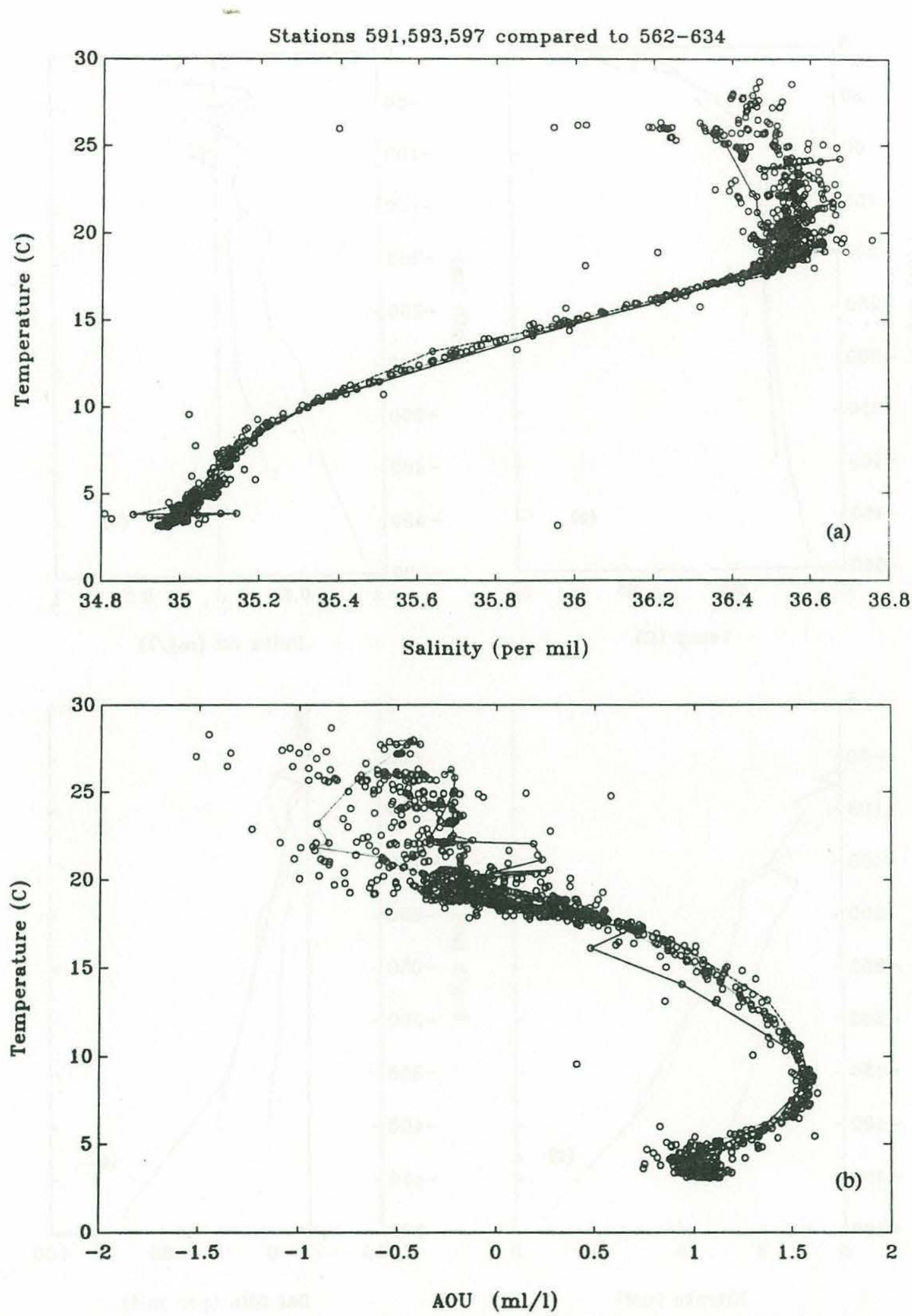

Figure 4-2: Property vs. temperature plots for: (a) salinity, (b) AOU, (c) nitrate, (d) $\delta^{3} \mathrm{He}$ for the anomalous station 593 (solid line), the previous station (dotted line), the following station (dashed line), and all the 1985-1987 data (points). 

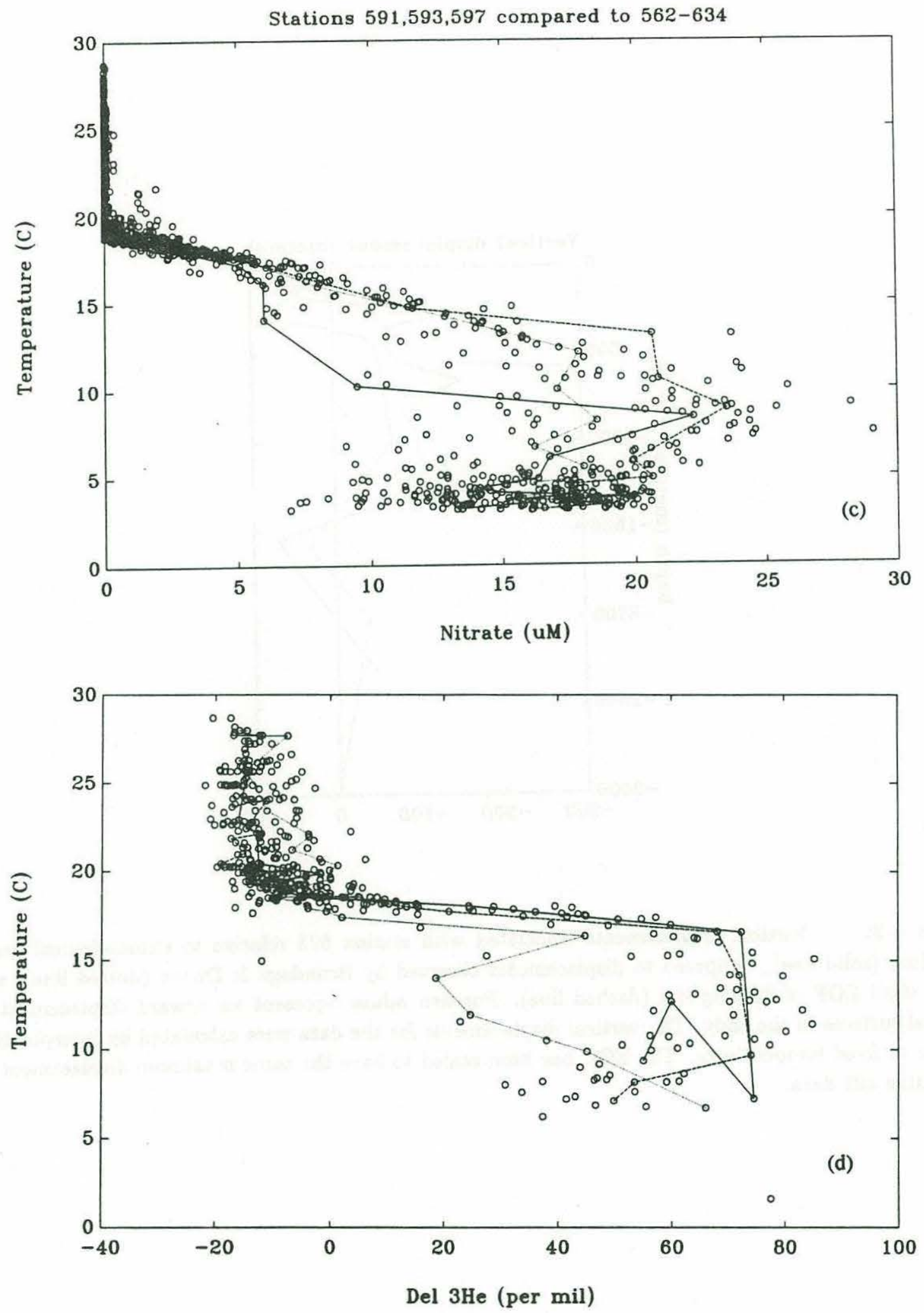

Figure 4-2: (continued) 


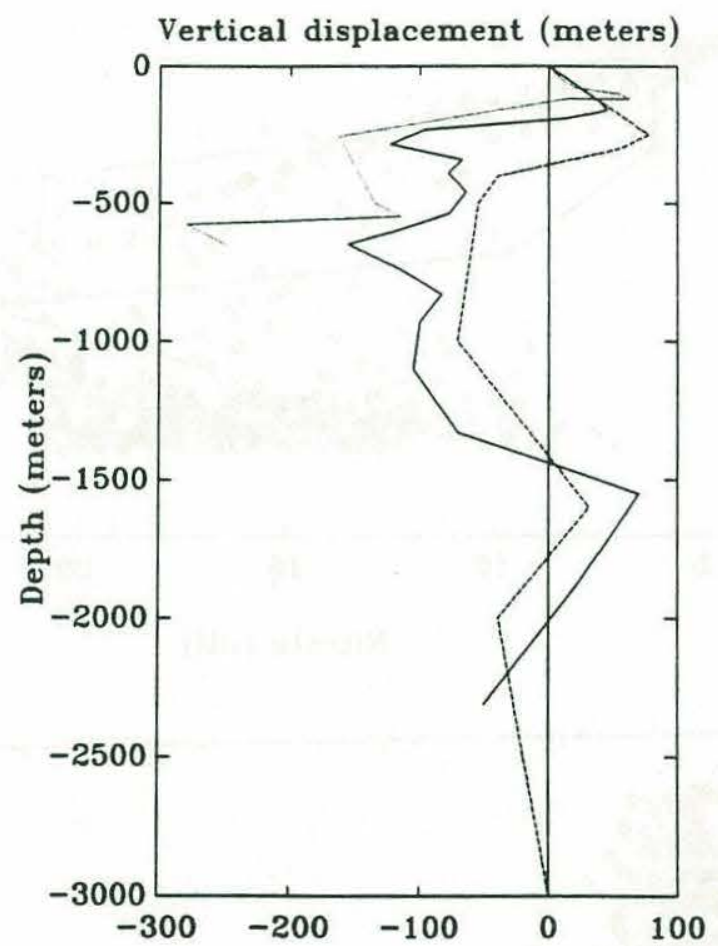

Figure 4-3: $\quad$ Vertical displacements associated with station 593 relative to climatological mean conditions (solid line), compared to displacements observed by Brundage \& Dugan (dotted line), and to the third EOF of Frankignoul (dashed line). Positive values represent an upward displacement of material surfaces in the eddy. The vertical displacements for the data were calculated by interpolating to a set of fixed temperatures. The EOF has been scaled to have the same maximum displacement as the station 593 data. 

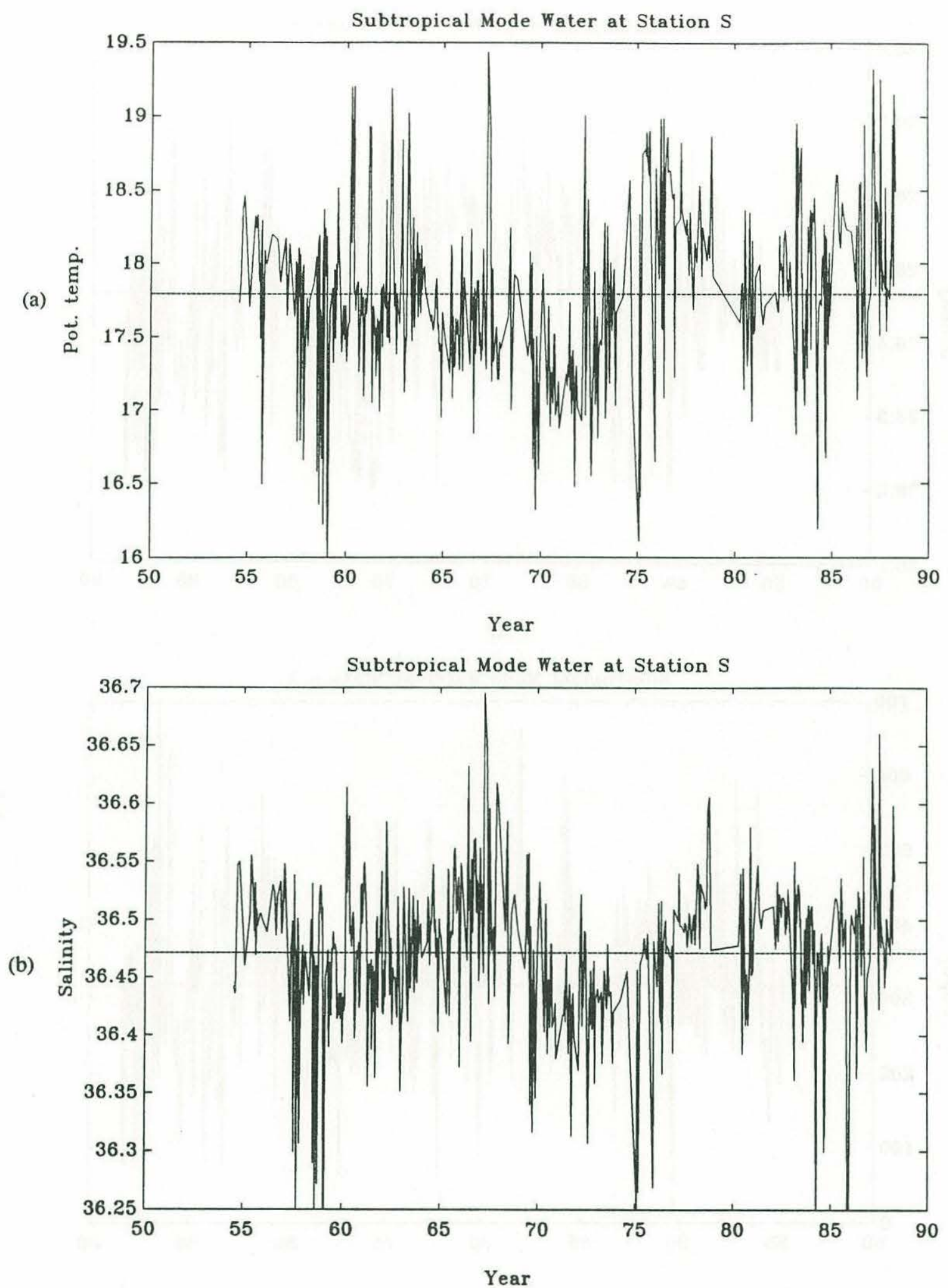

Figure 4-4: Properties of the subtropical mode water core for 1955-1987 at Station S: (a) potential temperature, (b) salinity, (c) density, (d) depth of the core, (e) AOU, (f) potential vorticity. 

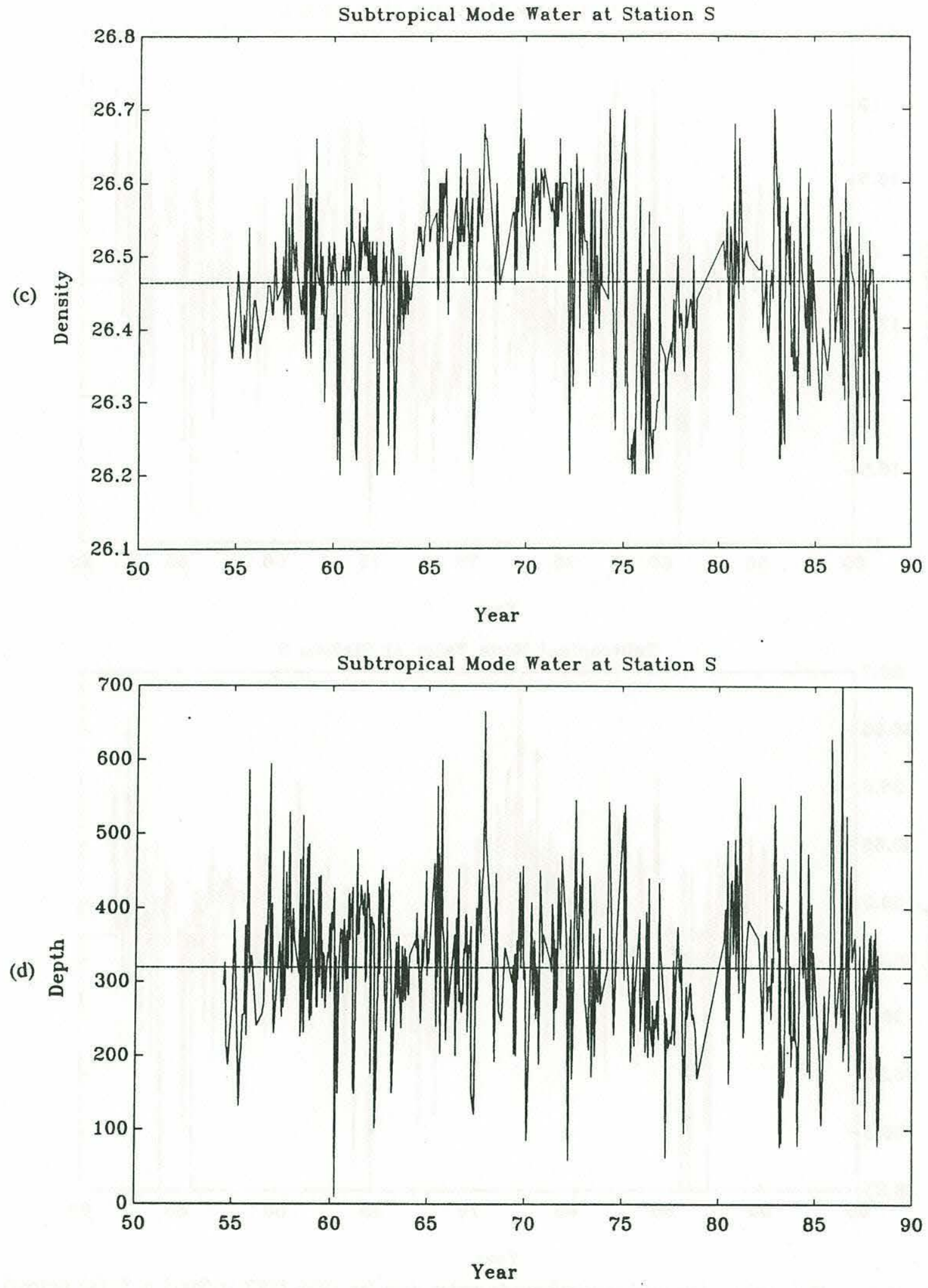

Figure 4-4: (continued) 
Subtropical Mode Water at Station S
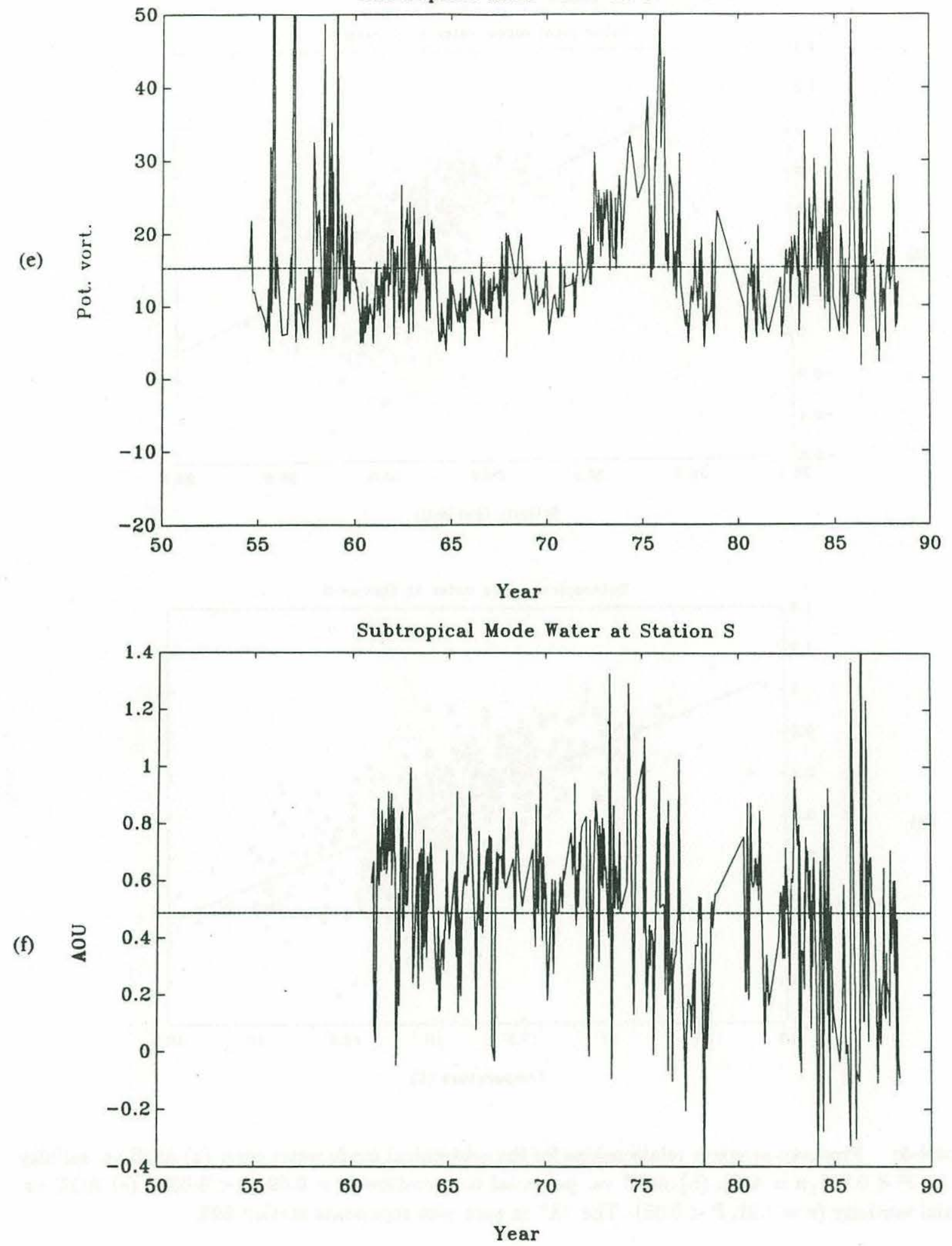

Figure 4-4: (continued) 
(a)

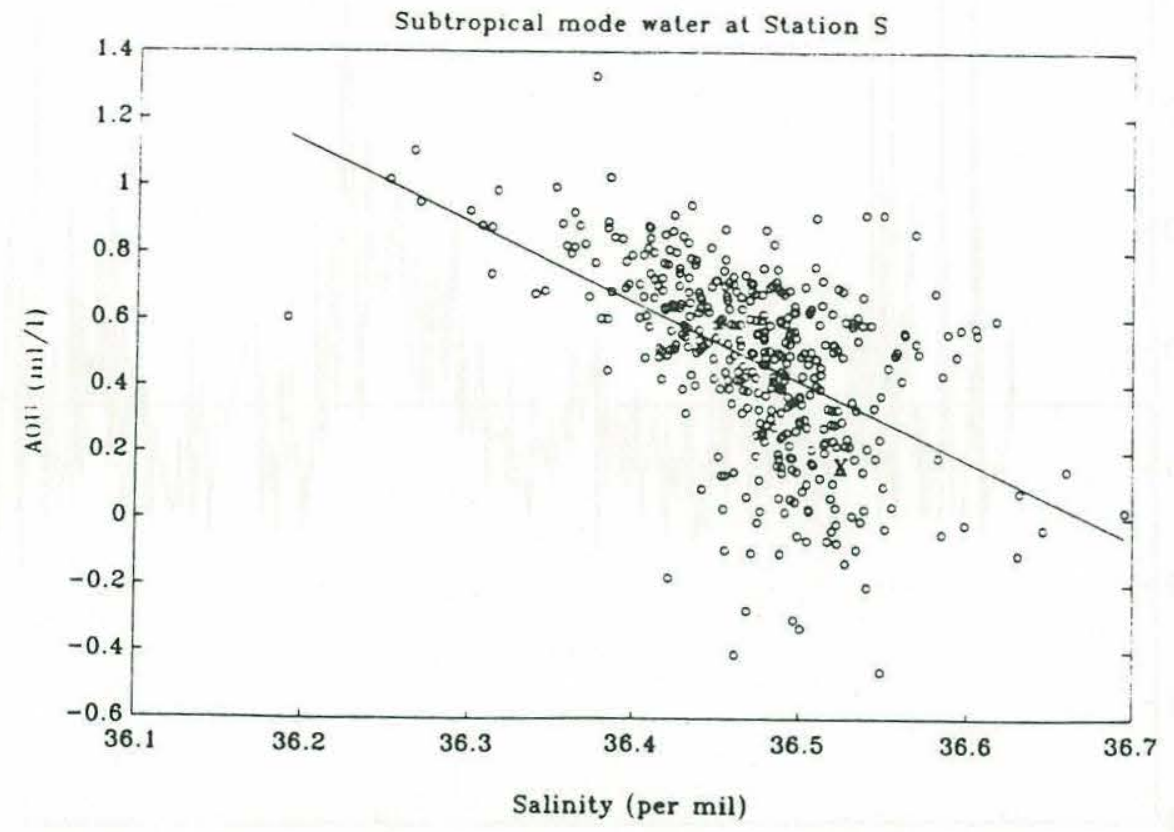

(b)

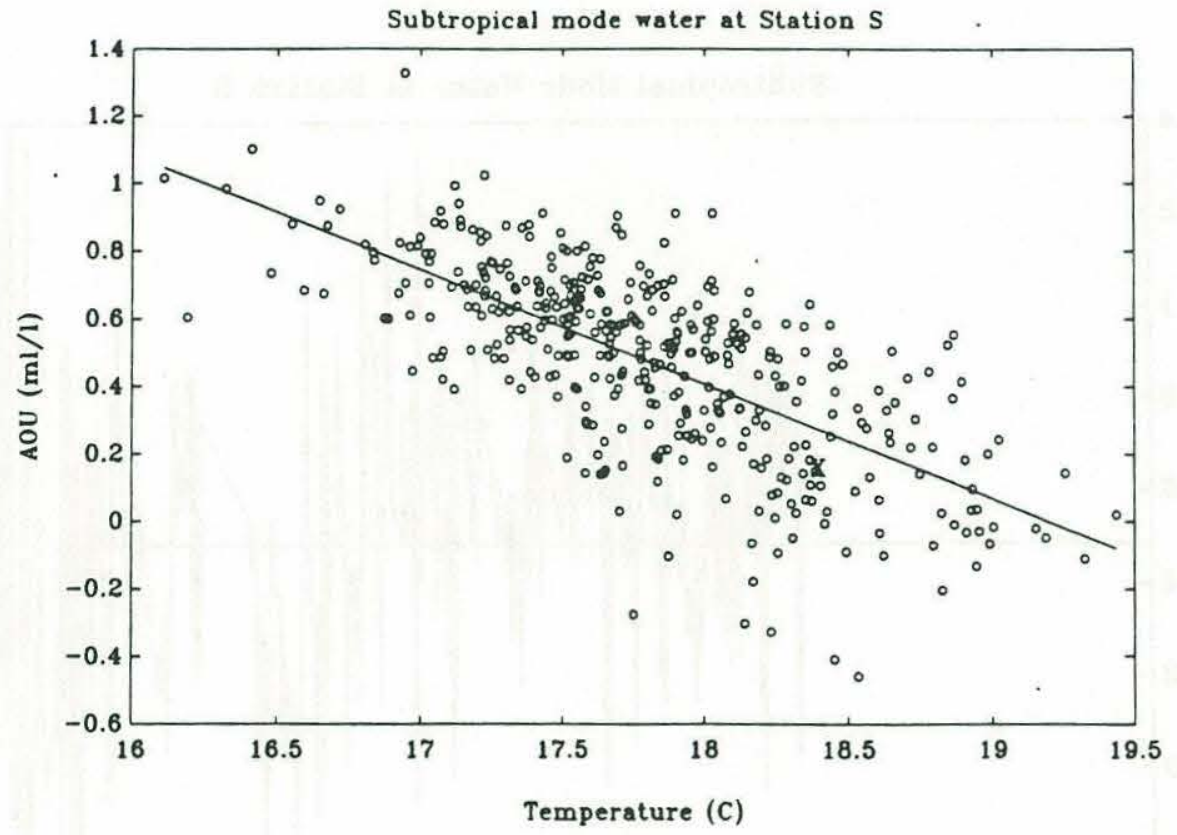

Figure 4-5: Property-property relationships for the subtropical mode water core: (a) AOU vs. salinity ( $r=.52, P<0.001, n=407)$, (b) AOU vs. potential temperature $(r=0.69, P<0.001)$, (c) AOU vs. potential vorticity $(r=0.21, P<0.05)$. The "X" in each plot represents station 593 . 
(c)

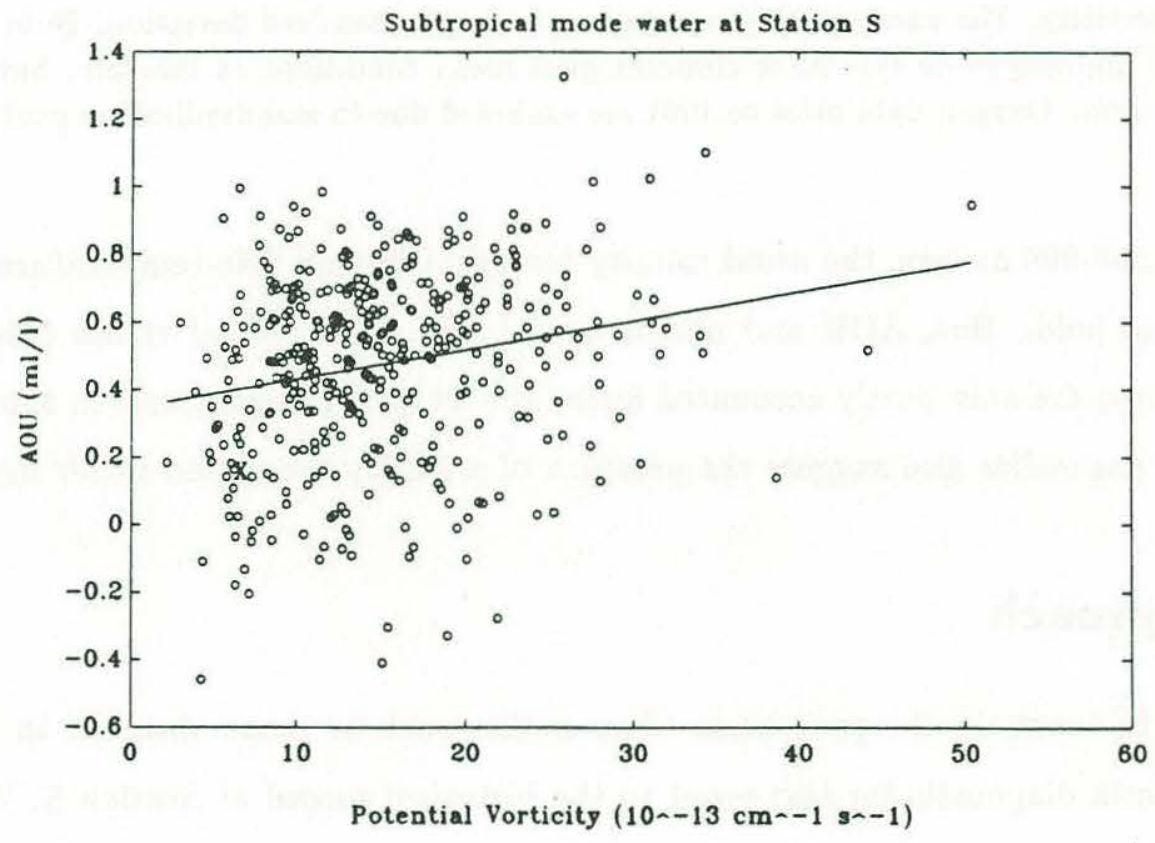

Figure 4-5: (continued) 


\begin{tabular}{lccc} 
Property & Sta 593 & 1986 Mean & 1954-1988 Mean \\
\hline $\mathrm{N}$ & 3 & 20 & 528 \\
$\theta\left({ }^{\circ} \mathrm{C}\right)$ & $18.37 \pm 0.03$ & $18.02 \pm 0.59$ & $17.79 \pm 0.56$ \\
$\mathrm{~S}(\% \circ \mathrm{\circ})$ & $36.52 \pm 0.01$ & $36.48 \pm 0.06$ & $36.47 \pm 0.06$ \\
$\sigma_{\theta} \% \circ \circ$ & $26.36 \pm 0.01$ & $26.43 \pm 0.11$ & $26.46 \pm 0.11$ \\
$\mathrm{AOU}\left(\mathrm{ml} \mathrm{l}^{-1}\right)$ & $0.11 \pm 0.14$ & $0.51 \pm 0.49$ & $0.49 \pm 0.30$ \\
$\mathrm{Q} 10^{-13}\left(\mathrm{~cm}^{-1} \mathrm{~s}^{-1}\right)$ & $6.18 \pm 1.2$ & $17.1 \pm 7.8$ & $15.3 \pm 9.7$ \\
$\mathrm{z}(\mathrm{meters})$ & 264 & $338 \pm 39$ & $320 \pm 98$ \\
$\delta^{3} \mathrm{He}(\% \circ)$ & 6.5 & $8.0 \pm 8.3$ & - \\
Nitrate $(\mu \mathrm{M})$ & 2.1 & $3.2 \pm 1.2$ & - \\
$17^{\circ} \mathrm{C}$ isotherm depth (meters) & 522 & $465 \pm 27$ & $458 \pm 54$ \\
$19^{\circ} \mathrm{C}$ isotherm depth (meters) & 95 & $155 \pm 33$ & $136 \pm 54$ \\
$17-19^{\circ} \mathrm{C}$ thickness (meters) & 426 & $310 \pm 41$ & $321 \pm 63$ \\
\hline
\end{tabular}

Table 4-1: Properties of the subtropical mode water at Station S, identified by a local minimum in potential vorticity. The uncertainties represent one sample standard deviation. Note that the mode water in 1986 appears to be typical of climatological mean conditions at this site, but station 593 is distinctly different. Oxygen data prior to 1961 are excluded due to standardization problems.

Between 500-900 meters, the usual salinity-temperature and ${ }^{3} \mathrm{He}$-temperature relationships also appear to hold. But, AOU and nitrate are at half their normal values (Figure 1-2) and these differences are only partly accounted for by the warmer temperatures in this depth range. These tracer anomalies also suggest the presence of a freshly ventilated water mass.

\subsection{Approach}

We attempt to ascertain the prevalence of anomalies such as those observed in July, 1986 by applying criteria diagnostic for this event to the historical record at Station S. We choose the thickening of the subtropical mode water as a "signature" for this type of eddy. We calculate the core properties of the mode water using the method of Talley \& Raymer (1982) to identify a local subsurface minimum in the density gradient. We focus on the potential vorticity minimum since this accounts for climatological modulation in the temperature and depth of the mode water. We obtain nearly identical results by using the $17-19^{\circ} \mathrm{C}$ isotherm thickness or the $200-400$ meter density gradient to measure the vertical extent of this layer.

The 35 year record at this site consists of temperature, salinity, and oxygen measured at standard depths with an average frequency of 18 times per year since 1954 (WHOI \& BBSR, 1988). We estimate an upper limit to the precision of the temperature measurements of $\pm 0.03^{\circ} \mathrm{C}$ 
for a given station and $\pm 0.07^{\circ} \mathrm{C}$ for the entire timeseries, based on the reproducibility of measurements during 1955-1987 at a depth of $2000 \pm 200$ meters where natural variations are presumed to be small. We estimate a maximum uncertainty of $\pm 1 \%$ in the depths (determined with reversing thermometers). Including errors in linear interpolation, the resulting error in potential vorticity is less than $\pm 20 \%$. An upper limit to the error in AOU is $0.14 \mathrm{ml} \mathrm{l}^{-1}$ from similar considerations (Spitzer \& Jenkins, 1989) ${ }^{4}$.

We employ spectral analysis to further characterize the variations in potential vorticity and AOU. The timeseries is sampled at somewhat irregular intervals, so we linearly interpolate in the time domain to yield a series sampled at 1024 evenly spaced intervals. We then low-pass filter and decimate this series to yield a 512-point series. We estimate the spectra using the Welch method with conventional fast-Fourier transform techniques. We achieve comparable results by analyzing the original data with an algorithm designed for unevenly sampled timeseries (Lomb, 1976; Scargle, 1982), which suggests that aliasing by higher frequencies (up to twice the average Nyquist frequency of $9 \mathrm{yr}^{-1}$.

\subsection{Results}

The timeseries of mode water properties (Figure 1-4) indicate significant decade-timescale variations as well as large high-frequency fluctuations. The long-term variations appear to reflect changes in the process of mode water formation as discussed by Jenkins (1982) and Talley \& Raymer (1982). A maximum in water mass renewal rates in the mid-1960's is followed by a minimum in the early 1970 's. After the severe winter of 1976-1977, renewal rates return to values similar to their climatological mean values. Periods with high renewal rates are characterized by mode water with high temperature, high salinity, low potential vorticity, and low AOU. Thus, the AOU of the mode water is moderately well-correlated with salinity as pointed out by Jenkins (1982), although the correlation with temperature is somewhat better (Figure 1-5).

The short-term fluctuations suggest that anomalies such as those observed in July, 1986 are not uncommon. On average, there are about 1-2 events per year with reductions (relative to climatological mean values) in potential vorticity and AOU of amplitude greater than or equal to

\footnotetext{
${ }^{4}$ Oxygen data prior to 1961 are excluded due to standardization problems (Jenkins,1982).
} 
that observed in July, 1986; there are 4-5 per year of half that amplitude. However, these events appear to be clustered during years of vigorous mode water formation (Figure ??). Furthermore, there is a seasonal bias; events with low potential vorticity and AOU occur predominantly during late spring and summer. Jenkins (1982) has also noted that minima in density gradient and AOU on $\sigma_{\theta}=26.4$ occur during late spring-early summer. If the eddies are formed during late winter convection in the northeast Sargasso Sea, the timing of their arrival at Station $\mathrm{S}$ is consistent with an advective transit time associated with the mean gyre recirculation.

The power spectra of potential vorticity and AOU in the mode water (Figure ??) are consistent with the time-domain trends discussed above. Most of the power is at at lowfrequencies, suggesting the dominance of decade-timescale climatic variations in determining the properties of the mode water. The annual cycle of mode water formation results in a peak at this frequency in the raw (unbinned) spectra, although it is not clearly resolved in the binned spectra. There are significant peaks at higher frequencies as well, in the band 2-6 $\mathrm{yr}^{-1}$. The variations in potential vorticity and AOU in each of these frequency bands are coherent and in phase as expected from the time-domain trends. We suggest that the high frequency variations are caused by events such as the one observed at station 593. Their frequency of occurrence is consistent with our estimates from the time domain trends, and suggests that anticyclonic eddies in the mode water may arrive at Station $S$ with an average frequency of 2-6 times per year.

We gain additional support for these inferences by examining the results of Frankignoul (1981), who subjected the temperature record at this site (1954-1978) to empirical orthogonal function (EOF) analysis. The eddy field is dominated by two EOF's (contributing a total of $83 \%$ of the variance) which resemble the first two baroclinic normal modes. A third EOF contributes $8 \%$ of the total variance, and is is strikingly similar to the eddy structure described above (Figure 1-3). Furthermore, the power spectrum of this EOF is similar to those presented above and contains a significant peak in the band 1-3 $\mathrm{yr}^{-1}$.

Our estimate of the frequency of eddies may be somewhat conservative because our sampling rate is comparable to the duration of the events. With a typical horizontal scales of $100 \mathrm{~km}$ and a translational speed of order $4 \mathrm{~cm} \mathrm{~s}^{-1}$, an eddy whose center passes directly through the Station S site will be observable for about 25 days. The average sampling interval is about 21 

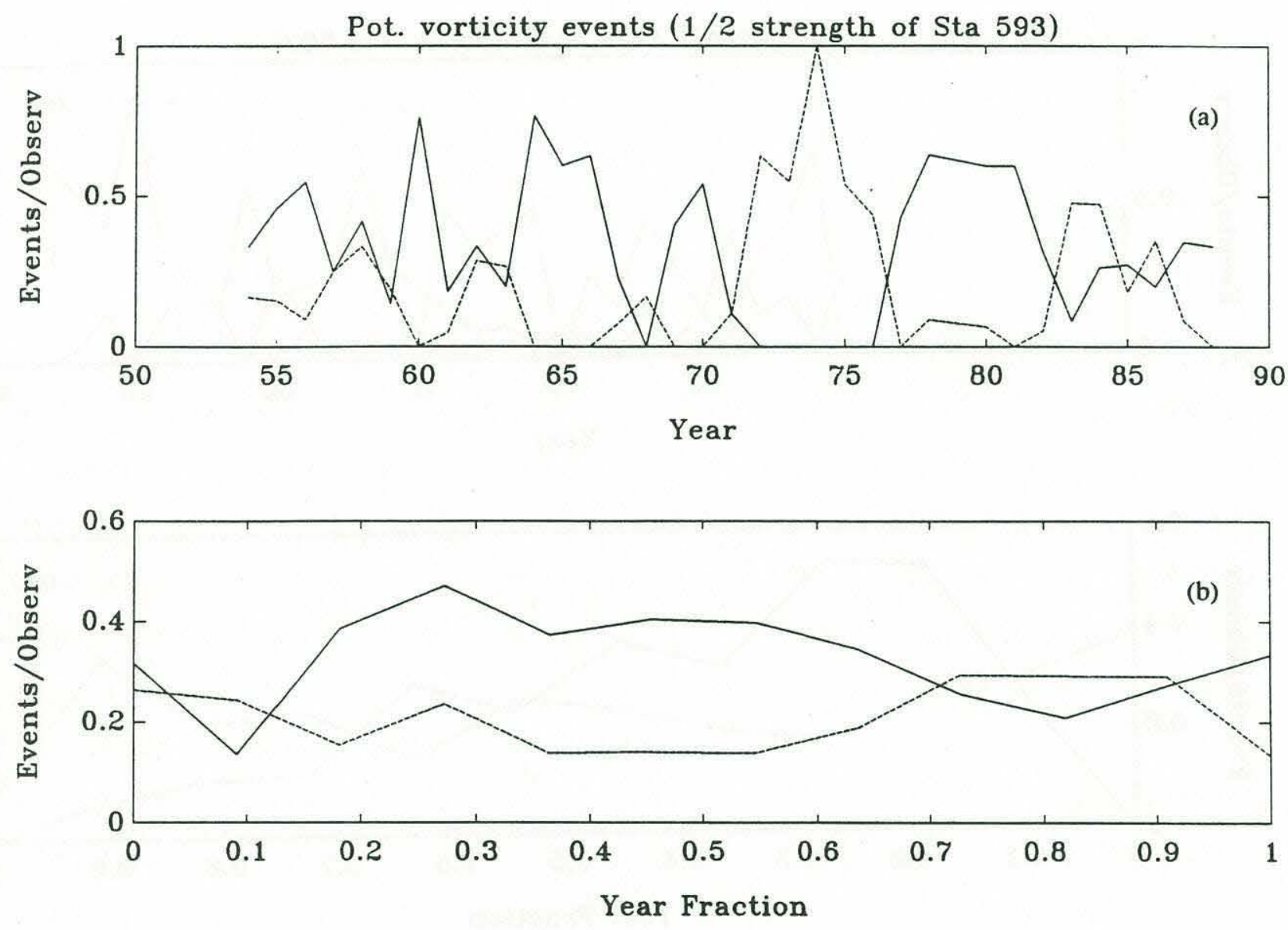

Figure 4-6: Distribution of events with low (solid line) and high (dotted line) potential vorticity: (a) frequency of events per year (each year starts on April 1), (b) frequency of events per month. An event is defined as a departure from the climatological mean value greater than or equal to half that observed at station 593. Distribution of events with low (solid line) and high (dotted line) AOU: (c) frequency of events per year, (d) frequency of events per month. 

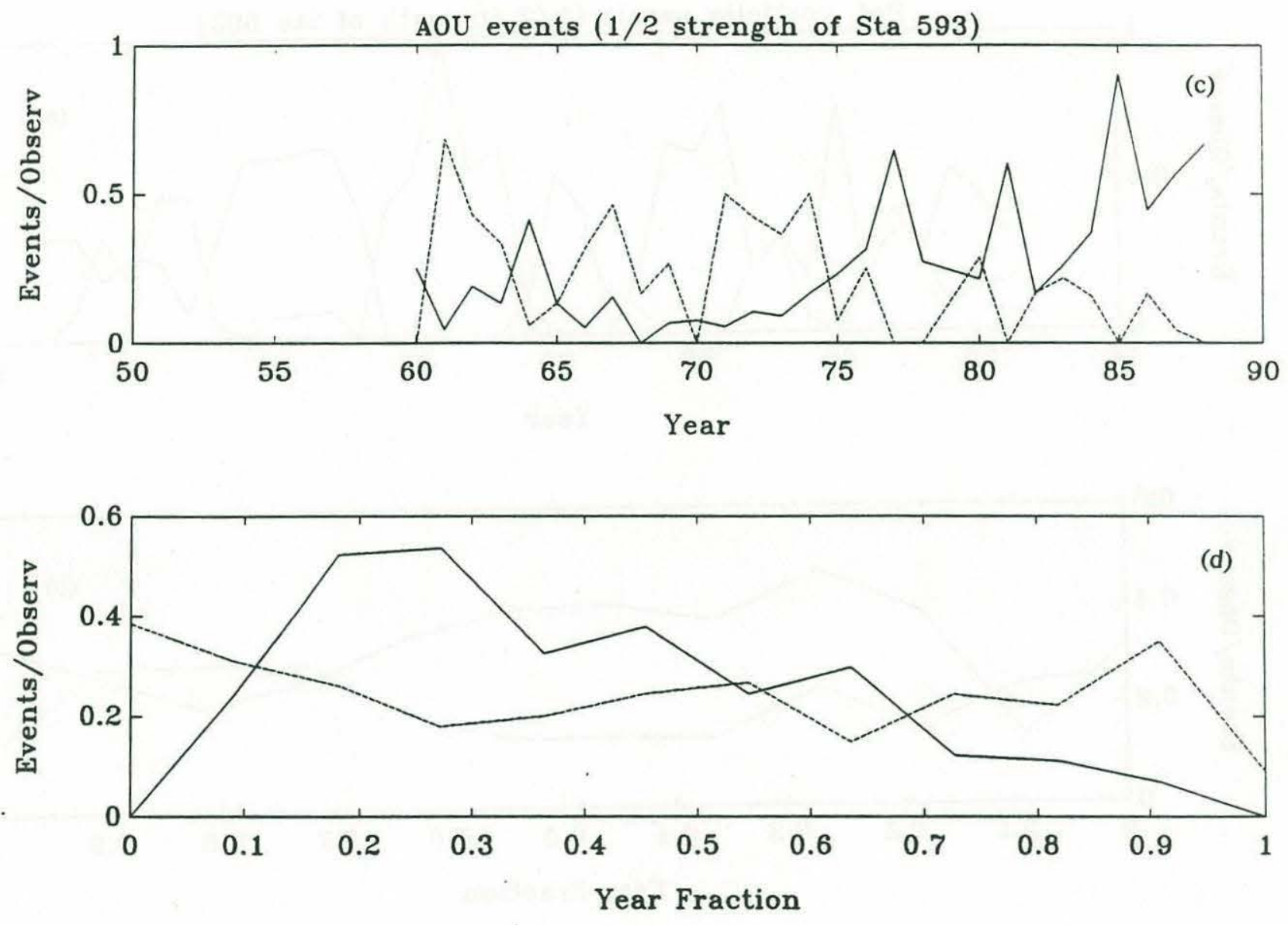

Figure 4-6: (continued) 

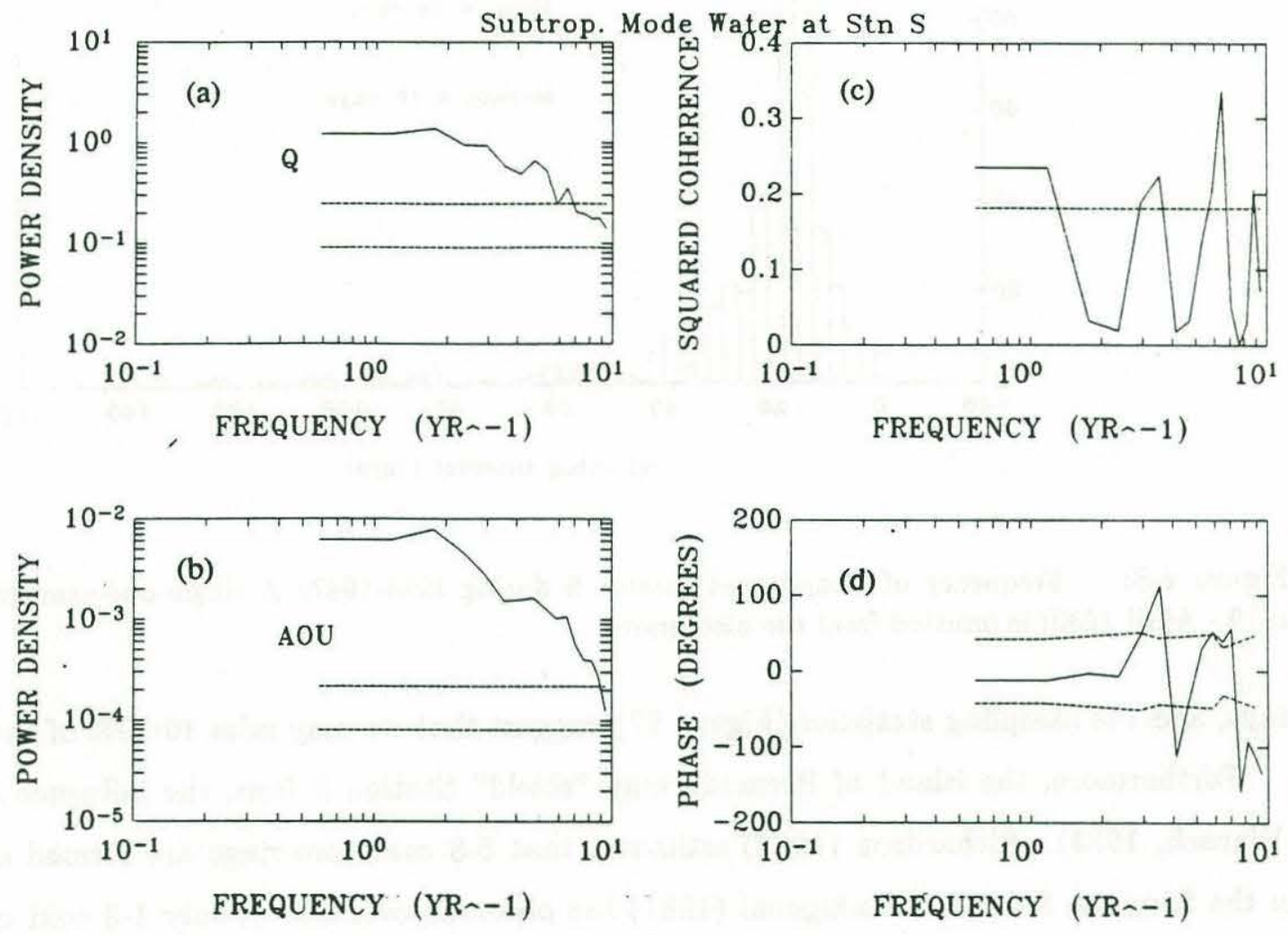

Figure 4-7: Power spectra for (a) potential vorticity and (b) AOU, and cross-spectral (c) coherence and (d) phase. The dashed lines represent $95 \%$ confidence intervals for both power spectra and the cross-spectral phase, and indicate the $95 \%$ signficance level for coherence. 


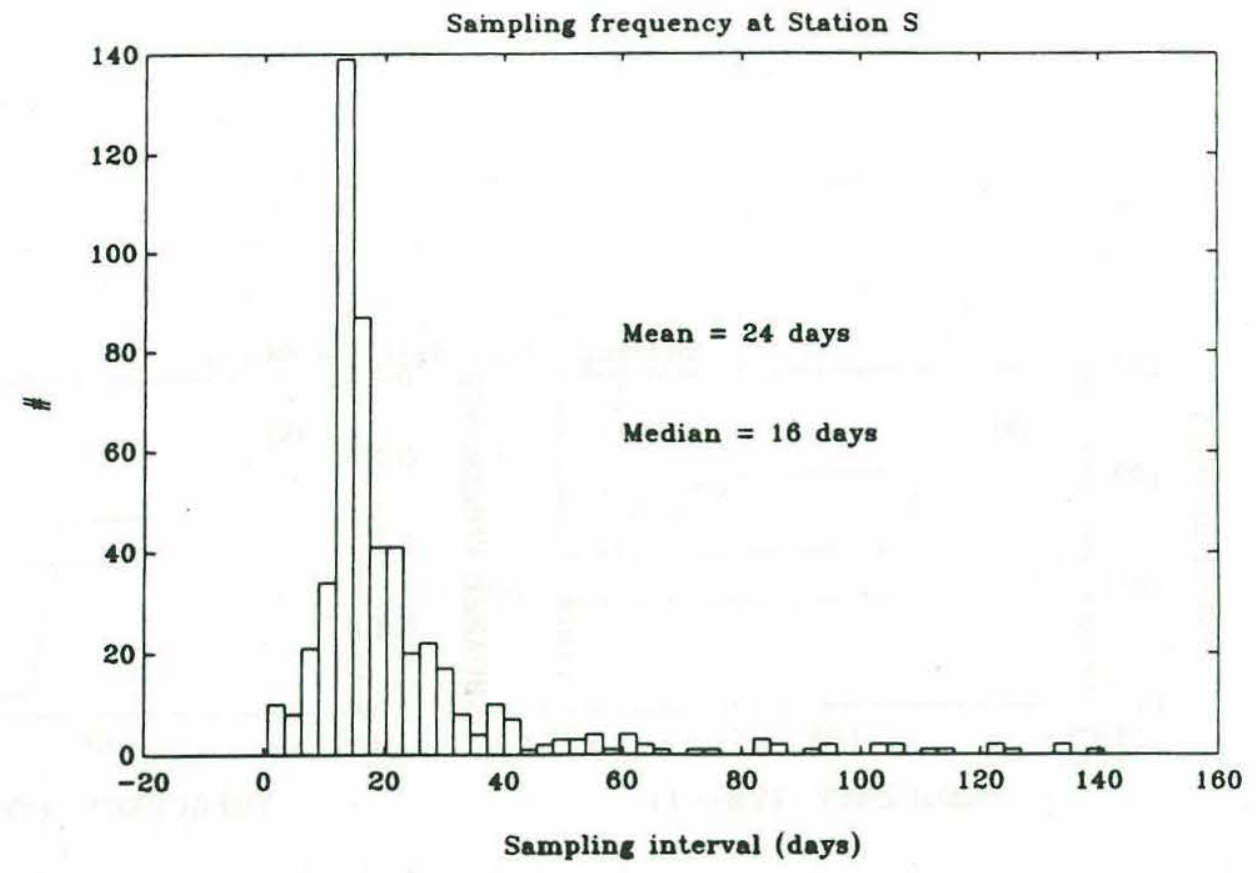

Figure 4-8: Frequency of sampling at Station S during 1955-1987. A single one-year gap (March 1979 - April 1980) is omitted from the histogram.

days, and the sampling statistics (Figure ??) suggest that we may miss $10-20 \%$ of the events.

Furthermore, the island of Bermuda may "shield" Station S from the influence of eddies (Wunsch, 1983). Richardson (1983) estimates that 5-8 cold core rings are formed each year in the Sargasso Sea, yet Frankignoul (1981) has observed evidence of only 1-3 cold core rings during 25 years (1954-1978) of hydrographic measurements at Station S. The apparent "deficit" at Station $\mathrm{S}$ suggests that we may be drastically estimating the population of the mode water eddies. On the other hand, it is possible that the location of Station S favors the detection of the mode water eddies in which case we could be overestimating the true eddy population.

\subsection{Formation mechanism}

The above patterns are consistent with simple descriptions of mode water formation (Talley \& Raymer, 1982; Jenkins, 1982), and implicate this process as a likely cause. At the start of the winter cooling cycle, a seasonal thermocline overlays much of the Sargasso Sea. Below are 
the remnants of the previous winter's mixed layers, displaced from their formation region by the gyre circulation. As cooling begins, the seasonal thermocline is eroded and the underlying pycnostads are exposed to further cooling, deepening, and freshening. In the northwest corner of the Sargasso Sea, these pycnostads are warmer and lighter than the subtropical mode water, having been recirculated from the south and east. As the pycnostads circulate eastward, they are further modified, and an east-west gradient in the pycnostad properties is maintained. Maps of ${ }^{3} \mathrm{H}-{ }^{3} \mathrm{He}$ age on the $\sigma_{\theta}=26.5$ surface (Jenkins, 1988a) indicate the progressive ventilation of this layer, with the apparent ventilation age decreasing downstream. One would also expect AOU to decrease in this direction. Severe events in late March produce the coldest and deepest mixed layers at each location; those formed just south of the Gulf Stream are termed subtropical mode water.

As the warming cycle begins, these pycnostads are capped off by a seasonal thermocline. The colder forms of mode water, formed in the northeast corner of the gyre, are recirculated to the southwest, and are observed beneath the local seasonal thermocline at Station $\mathrm{S}$ several months later. A possible "short circuit" (Talley \& Raymer, 1982) could result in a mid-spring arrival of a warmer, western form of mode water.

Since it is associated with severe weather, it is likely that the mode water formation occurs over horizontal scales characteristic of storms (order 100 kilometers). Storms can "punch through" the seasonal thermocline to form deep mixed layers; baroclinic adjustment may then form anticyclonic lenses, with cyclonic lenses below to conserve potential vorticity. These dynamic columns would then be advected by the gyre circulation, and would be observed at Station S later in the year as events with low potential vorticity and AOU.

Although it seems most likely that the formation of anticyclonic eddies is related to mode water formation, Brundage \& Dugan suggest an alternative formation mechanism: vortex shedding associated with interaction of the Gulf Stream with the Corner Rise Seamounts. The interannual and seasonal patterns make the former mechanism more compelling, but the vortex shedding mechanism may be more active during severe winters when the Gulf Stream transport is larger (Worthington, 1977). However, the tracer observations indicate that the eddy core is freshly ventilated and favor mode water formation as the probable cause (since the vortex shedding mechanism would probably not expose the mode water to the surface). 


\subsection{Tracer injection}

We observe that the mode water eddy in July, 1986 is associated with the injection of nitrate and

${ }^{3} \mathrm{He}$ into the layers above. By what mechanism could this occur, and how could it contribute to new production? If the eddies are dynamic columns that pass beneath the seasonal thermocline, then they may enhance the upward flux of these tracers by uplifting material surfaces into the euphotic zone. The resupply of the tracers via isopycnal mixing may be enhanced by intensified tracer gradients and velocity shear at the edge of the eddy; we can estimate a crude upper limit to this flux using typical nitrate concentrations $\left(2.5 \mu \mathrm{M}\right.$ on $\sigma_{\theta}=26.2$; Kawase \& Sarmiento, 1985) outside the eddy and assuming zero nitrate concentrations within. Using a mixing coefficient of $25 \mathrm{~m}^{2} \mathrm{~s}^{-1}$, obtained by scaling down large-scale diffusivity estimates (Armi \& Stommel, 1983; Jenkins, 1987) by a factor of $L^{4 / 3}$ (Okubo, 1971), we estimate that isopycnal mixing could supply less than $15 \%$ of the excess nitrate observed in July 1986 over a timescale of 3 months (the estimated advective transit time from the formation region to Station $\mathrm{S}$ ). Thus, another mechanism must be invoked to account for tracer transport into the eddy. Possibly, the interior dynamics of the eddy are responsible for the bulk of the influx; enhanced mixing via shear instability may play a role (Stern, 1987).

If the dynamic column extends to the surface, then the eddy dynamics may be analogous to those in a warm core ring, where upwelling in the center brings tracers closer to the surface (Joyce, 1988; Nelson et al., 1989). With estimated upwelling rates of order $1 \mathrm{~m}$ day $^{-1}$, the nutricline would be raised into the lower euphotic zone (but not into the summer mixed layer). However as shown by Joyce (1988), the energy required to "punch through" the stratification is reduced by up to two orders of magnitude in the eddy center. A high-wind event might then entrain the tracers into the mixed layer.

Goldman (1988) has hypothesized that a nutrient pulse to the lower euphotic zone may generate a large pulse of new production by initiating a bloom of opportunistic large phytoplankton (e.g. diatoms). Alternatively, a "seed population" living within the nutricline may respond to an upward displacement by taking advantage of the increased (from their perspective) irradiance. In either case, the response of the phytoplankton might temporarily exceed the capacity of grazing populations. The sinking of these cells (either due to buoyancy changes or grazing) would contribute to a new production flux from the euphotic zone. Goldman has 
argued that only large cells could sustain such a response since small phytoplankton are part of a very efficient food web involving small bacteria and zooplankton where little new production could escape.

The photosynthesis associated with such a pulse would leave behind an accumulation of oxygen, but this would be difficult to detect since the accumulation of oxygen below the mixed layer tends to integrate over short-term events. The nutrient pulse would vanish rapidly; the observation of excess nutrients at station 593 was probably fortuitous. Jenkins (1988b) estimated that the likelihood of observing the nutrients is order $1 \%$. However, the ${ }^{3} \mathrm{He}$ excess will tend to persist since it depends on vertical transport into the mixed layer and subsequent gas exchange loss. In fact, the excess observed at station 593 persists at least 50 days later until station 597 (Jenkins, 1988a).

\subsection{Comparison with other tracer sources}

How does the eddy-driven flux of ${ }^{3} \mathrm{He}$ and nitrate compare with other sources? If there are 2-6 eddies per year and each eddy provides order $5-15 \%$ of the annual flux at Station S, the annual contribution would be $10-80 \%$ of the total.

This flux turns out to be comparable to that sustained by local winter convection. We estimate an upper limit to the contribution of convection by assuming that the entire mixed-layer inventories of these tracers are ultimately lost (via biological uptake of nitrate and outgassing of ${ }^{3} \mathrm{He}$ ). We calculate these inventories using both the annual average tracer-depth relationship and the observed winter profiles for 1985-1987. The close agreement between these two estimates suggest that less than $20 \%$ of the nutrients in the winter profiles have been consumed prior to restratification later in the spring. This is consistent with the hypothesis that phytoplankton are unable to respond to the winter nutrient pulse until the onset of stratification allows them to remain in the upper layers where light is sufficient for photosynthesis.

The estimated tracer inventories in the winter mixed layer depend strongly on the assumed depth of winter convection (Figure ??). For the climatological mean conditions (winter mixing to 150 meters), we estimate that approximately $15 \%$ of the annual flux could be supported, with a range of 5-60\% accounting for interannual variation. An additional source of uncertainty is that the tracer-depth relationships may vary with climatological conditions (e.g. if tracers 
are continually "mined" from the thermocline, they might become depleted after several years).

Nevertheless, this calculation suggests that winter convection is probaby not the sole source of tracers to the upper layers. This is not surprising, since we know that although new production is at a minimum in summer it remains nonzero despite undetectable nitrate concentrations $(<0.03 \mu \mathrm{M})$ in the euphotic zone. Deuser's (1986) measurements of particle flux at 3200 meters suggest that the summer flux is usually only a factor of 2 less than that in the spring. In addition, the accumulation of oxygen in the summer thermocline indicates that production continues under stratified conditions during the warming season. These observations imply that nutrients are supplied to the euphotic zone even during the summer.

Additional pools (e.g. dissolved organic nitrogen, particulate organic nitrogen) could contribute to the effective vertical transport of nutrients; however, these would not explain the measured ${ }^{3} \mathrm{He}$ flux. Hamilton et al. (1989) have proposed that salt fingering may enhance the turbulent fluxes of dissolved constituents relative to that of temperature by a factor of two; however, this would still require unreasonably large apparent vertical diffusivities.

Thus, it appears plausible that mode water eddies may contribute substantially to the tracer flux into the euphotic zone, and the tracers could be resupplied either by isopycnal mixing or an upwelling/wind-event mechanism. Winter convection may also supply a comparable fraction of the annual fluxes. Vertical mixing could contribute only 10-20\% (accounting for enhancement due to salt fingering).

\subsection{Implications for mode water renewal \& new production}

These eddies must also contribute to the renewal of the $18^{\circ} \mathrm{C}$ water. The transport of ${ }^{3} \mathrm{He}$ and nitrate into the eddies will tend to decrease the mean ventilation age of the surrounding water. Moreover, if they are eventually reabsorbed by the Gulf Stream (likely if they are carried westward by the gyre recirculation), the eddies will mix freshly ventilated water into the mode water pool.

The tritium-helium age distribution of the mode water (Jenkins, 1988b) suggests that on average $20-30 \%$ of the mode water is renewed annually. Worthington (1976) has estimated the volume of $18^{\circ} \mathrm{C}$ water as approximately $0.9-1.7 \times 10^{6} \mathrm{~km}^{3}$. We estimate the volume of the 

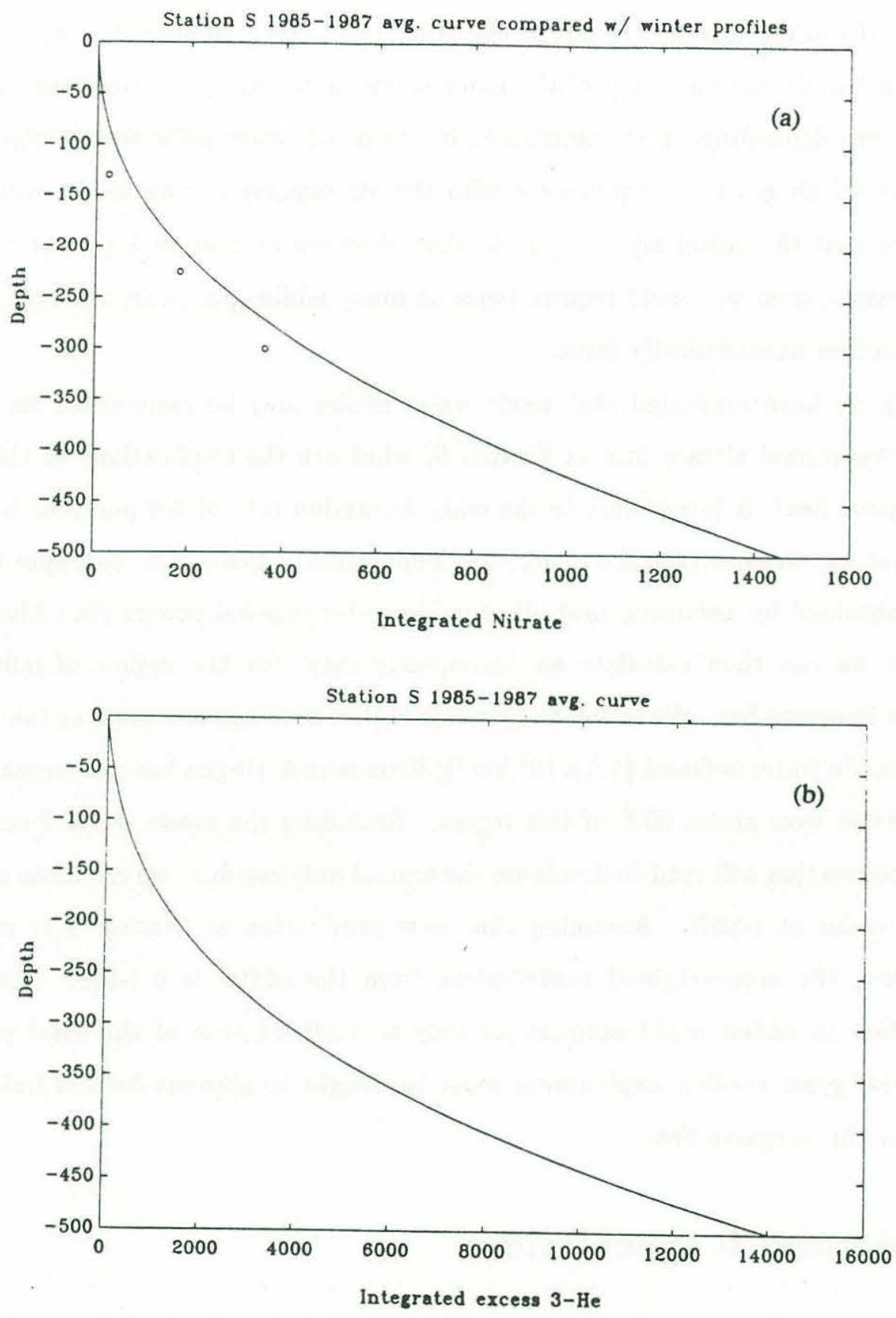

Figure 4-9: Integrated profiles of $(\mathrm{a})$ nitrate $(\mu \mathrm{M}-\mathrm{m})$ and $(\mathrm{b}) \delta^{3} \mathrm{He}(\% \circ \mathrm{o})$ from the surface to the indicated depth. Solid line is calculated from the mean depth profile during 1985-1987. Circles are calculated using only the late winter profiles. Estimated annual fluxes are $600 \pm 200 \mu \mathrm{M}$-m for nitrate and $6900 \pm 2200 \%$ oo-m for ${ }^{3} \mathrm{He}$. 
mode water in an eddy as approximately $0.6-1.2 \times 10^{4} \mathrm{~km}^{3}$ using the same criteria. If we assume that the initial age of the eddy is essentially zero, then $30-40$ eddies per year would be required to maintain the mean age of the mode water in steady-state. However, the initial age may be nonzero depending on the conditions in the mode water prior to convection as well as the extent to which gases can equilibrate with the atmosphere during mode water formation. If we assume that the initial age is equal to that observed at Station $S$ (about half the mean age of 3-5 years), then we would require twice as many eddies per year; however, this number of eddies becomes unrealistically large.

Although we have suggested that mode water eddies may be responsible for a significant fraction of the annual nitrate flux at Station S, what are the implications of this hypothesis for the Sargasso Sea? A lower limit to the eddy formation rate of 2-6 per year is obtained by assuming that we have detected the entire eddy population at Station S. An upper limit of 30-40 per year is obtained by assuming that all of mode water renewal occurs via eddies. Assuming steady-state, we can then calculate an "occupancy rate" for the region of influence of the eddies in the Sargasso Sea. We estimate that this region encompasses most of the region where subtropical mode water is found $\left(5.5 \times 10^{6} \mathrm{~km}^{2}\right)$; Brundage \& Dugan have observed anticyclonic mesoscale eddies over about $50 \%$ of this region. Excluding the mode water formation region where local convection will tend to dominate the annual nutrient flux, we calculate an occupancy rate on the order of $1-30 \%$. Assuming that new production at Station $\mathrm{S}$ is representative for this region, the area-weighted contribution from the eddies is $0.1-5 \%$. Thus, enhanced new production in eddies could account for only a small fraction of the total production in the subtropical gyre; another explanation must be sought to account for the bulk of the new production in the Sargasso Sea.

\subsection{Summary \& speculations}

We have suggested that anomalous hydrographic profiles observed in July, 1986 at Station S are caused by passage of a mesoscale anticyclonic eddy, and that this event could provide $5-15 \%$ of the annual ${ }^{3} \mathrm{He}$ and nitrate flux to the euphotic zone at this site. Analysis of the historical hydrographic record at this site suggests that such eddies pass Station $\mathrm{S}$ with an average frequency of 2-6 $\mathrm{yr}^{-1}$, and implies that they may be an important mechanism by which these 
tracers are supplied to surface waters, especially during the spring and summer and in years of vigorous mode water formation. Moreover, they may represent a significant pathway by which freshly ventilated mode water is incorporated into the gyre. However, enhanced new production in such eddies could account for only a small portion of the new production integrated over the Sargasso Sea.

The arguments we have presented are largely based on circumstantial evidence. In order to further test our hypothesis, we require more information about the detailed structure and evolution of these eddies. These features might be detected by remotely-sensed measurements of dynamic height anomaly (an eddy would produce a $5-10 \mathrm{~cm}$ displacement) or surface color (if biomass is enhanced near the surface), although this remains to be seen. Diagnostic model experiments could aid in understanding their dynamics. 


\section{Chapter 5}

\section{Summary}

\subsection{Analytical developments}

I have developed an analytical technique to measure argon concentrations in seawater by isotope dilution mass spectrometry, with a systematic uncertainty of less than $\pm 0.2 \%$. Analytical precision is $\pm 0.3 \%$, although reproducibility of samples from Station $S$ is inflated to approximately $\pm 0.6 \%$ by errors associated with sampling. These errors, apparently related to trapping of air bubbles, could be reduced by developing a method where no headspace is introduced while drawing the sample. Such a technique employing a collapsible container is being developed as part of the World Ocean Circulation Experiment (WOCE).

\subsection{Seasonal gas cycles in the upper ocean}

By measuring the response of the noble gases to the seasonal cycles of thermal and wind forcing, I estimate the rates of physical processes affecting gases in the upper ocean, and constrain their dependence on this forcing. Argon is quite sensitive to the gas exchange rate and somewhat sensitive to vertical mixing. Helium adds sensitivity to air injection processes. Temperature data is used to tightly constrain the apparent rate of vertical mixing.

The seasonal cycle of argon near Bermuda demonstrates unequivocally that only a small fraction of the subsurface oxygen supersaturation maximum can be attributed to physical causes, and therefore that most of the supersaturation must be attributed to photosynthetic oxygen 
production. Furthermore, the evolution of argon concentration in the seasonal thermocline suggests that most of the oxygen produced there is also lost due to vertical mixing and gas exchange.

In the winter mixed layer, air injection produces argon supersaturations despite high gas exchange rates. During spring and summer, radiative heating, air injection, and an upward argon flux create an even larger supersaturation in the mixed layer. In the seasonal thermocline, radiative heating maintains argon concentrations above solubility equilibrium in spite vertical mixing.

\subsection{Quantitative estimates of vertical mixing, gas exchange, and new production}

I have combined the noble gas, oxygen, and hydrographic data with an upper ocean model to place quantitative constraints on both the physical processes and the biological production of oxygen. The subsurface evolution of argon concentration requires a vertical diffusivity of $1.0 \pm 0.1 \times 10^{-4} \mathrm{~m}^{2} \mathrm{~s}^{-1}$, consistent with that derived from temperature. Measurements of argon and helium supersaturation determine the rate of air injection to $\pm 15 \%$ and the seasonallyaveraged gas exchange rate to $\pm 11 \%$. The inferred rate of bubble trapping is roughly consistent with previous work by Atkinson (1973), Thorpe (1984; 1986), and Crawford and Farmer (1987), and this process is dominated by partial trapping as suggested by Jenkins (1988b). Our estimate of gas exchange rate is consistent within errors with that predicted from climatological wind speeds and Liss \& Merlivat's (1986) formulation of piston velocity. A lower limit to new

production of oxygen is estimated as $5.0 \pm 1.0$ moles $\mathrm{m}^{2} \mathrm{yr}^{-1}$, and the uncertainty in the estimate might be reduced with higher quality oxygen data. If the near-surface new production is fixed at zero, I estimate $6.2 \pm 0.9$ moles $\mathrm{O}_{2} \mathrm{~m}^{2} \mathrm{yr}^{-1}$. Our estimate is close to the results obtained by J\&G and Musgrave et al. (1988) for the 1960-1970 period, and the 1985-1987 period appears to be representative of climatological mean conditions.

I have used the model to explore the sensitivity of our results to additional measurements of inert gases. These tests suggest that I could improve the annual estimates of gas exchange slightly by measuring $\mathrm{Kr}$ (and possibly Xe, although its solubility is not known as precisely). 
By measuring Ne, I could improve the estimate of air injection amplitude and also better constrain the mode of bubble trapping. Interestingly, $\mathrm{N}_{2}$ proves to have sensitivities generally comparable to $\mathrm{Ne}$, with a somewhat higher sensitivity to the trapping mode. Measurements of helium and neon during 1987 to be carried out in the near future will help to further constrain the amplitude and mode of bubble trapping.

The data obtained so far suggest that I am just barely resolving the vertical structure and temporal evolution of the gas distributions. More frequent sampling would help to better constrain the mixed layer balances, and better vertical resolution would help to resolve the subsurface maxima and improve the statistics of the mixed layer averages. CTD- $\mathrm{O}_{2}$ profiles would enhance vertical resolution, provide better hydrographic measurements, and allow more knowledgeable sampling of subsurface features. Improved oxygen measurements would permit more precise estimates of biological production.

\subsection{Upward flux of nutrients via eddies}

I have also attempted to understand how nutrients are supplied to the euphotic zone to support the observed levels of new production. Jenkins has shown that the upward nitrate flux is consistent with oxygen-based new production estimates. However, rates of apparent vertical mixing consistent with the noble gas, oxygen, and temperature cycles are much too small to satisfy this flux.

I have suggested that anomalous hydrographic profiles observed in July, 1986 at Station S are caused by passage of a mesoscale anticyclonic eddy, and that this event could provide $5-15 \%$ of the annual ${ }^{3} \mathrm{He}$ and nitrate flux to the euphotic zone at this site. Analysis of the historical hydrographic record at this site suggests that these eddies pass Station $\mathrm{S}$ with a an average frequency of 2-6 $\mathrm{yr}^{-1}$, and implies that they may be an important mechanism by which these tracers are supplied to surface waters, especially during the spring and summer and in years of vigorous mode water formation. Moreover, they may represent a signficant pathway by which freshly ventilated mode water is incorporated into the gyre. However, enhanced new production in such eddies could account for only a small portion of the new production integrated over the Sargasso Sea.

These arguments regarding the eddy hypothesis are largely based on circumstantial evidence. 
In order to further test these ideas, I require more information about the detailed structure and evolution of these eddies. These features might be detected by remotely-sensed measurements of dynamic height anomaly (an eddy would produce a $5-10 \mathrm{~cm}$ displacement) or surface color (if biomass is enhanced near the surface), although this remains to be seen. Diagnostic model experiments could aid in understanding their dynamics.

One approach would be to attempt to find one of these eddies soon after its formation, and then to document its subsequent evolution. Initial detection might be achieved by a largescale XBT survey of the probable formation region, the northeastern North Atlantic. Once an eddy is found, it could be seeded with neutrally buoyant floats and subsequently tracked via satellite. Its structure could be mapped in detail, and the evolution of its biological, chemical, and physical properties could be followed as it travels through the subtropical gyre. 


\section{Chapter 6}

\section{Bibliography}

Altabet, M.A. \& J.J. McCarthy (1986) Temporal and spatial variations in the natural abundance of ${ }^{15} \mathrm{~N}$ in PON from a warm-core ring. Deep Sea Res. 32, 755-772.

Armi, L. \& H. Stommel (1983) Four views of a portion of the North Atlantic subtropical gyre. J. Phys. Ocean. 13, 828-857.

Atkinson, L.P. (1973) The effect of air bubble solution on air-sea gas exchange. J. Geophys. Res. 78, 962-968.

Benson, B.B. (1965) Some thoughts on gases dissolved in the oceans. In: Proc. Symp. on Mar. Geochem. (eds. D.R. Schink and J.T. Corless), Univ. of Rhode Island Occasional Publ. No. 3, 91-107.

Bieri, R.H. (1974) Dissolved Conservative Gases in Seawater. In The Sea. Interscience, NY, Vol. 5, Chap. 6.

Boerboom, A.J.H. \& G. Kleyn (1969) Diffusion Coefficients of Noble Gases in Seawater. J. Chem. Phys. 50, 1086-1088.

Brettel, T.A. \& R.L. Grob (1985) Cryogenic techniques in gas chromatography. Amer. Laboratory Oct. 1985, 19-32.

Brink, K.H. (1989) Evidence for wind-driven current fluctuations in the western North Atlantic. J. Geophys. Res. 94, 2029-2044.

Broecker, H.C. \& W. Siems (1984) The role of bubbles for gas transfer water to air at higher wind speeds. Experiments in the wind-wave facility in Hamburg. In: Gas Transfer at Water Surfaces (eds. W. Brutsaert \& G.H. Jirka). Reidel, NY.

Broecker, W.S. \& T.H. Peng (1982) Tracers in the Sea. Eldigio Press, Palisades, N.Y., 690 pp. 
Broecker, W.S. et al. (1986) Isotopic versus micrometeorological ocean $\mathrm{CO}_{2}$ fluxes: a serious conflict. J. Geophys. Res. 91, 10517-10527.

Brundage, W.L. \& J.P. Dugan (1986) Observations of an anticyclonic eddy of $18^{\circ} \mathrm{C}$ water in the Sargasso Sea. J. Phys. Oceanogr. 16, 717-727.

Bunker, Andrew F. (1975) Energy exchange at the surface of the western North Atlantic ocean. WHOI Technical Report 79-3, 28 pp.

Carpenter, E.J. \& J.S. Lively (1980) Review of estimates of algal growth using ${ }^{14} \mathrm{C}$ tracer techniques. In Primary Productivity in the Sea (ed. P.G. Falkowski). Plenum Press, N.Y., $531 \mathrm{pp}$.

Carritt, D.E. \& J.H. Carpenter (1966) Comparison and evaluation of currently employed modifications of the Winkler Method for determining dissolved oxygen in seawater; NASCO report. J. Mar. Res. 24, 286-318.

Chou, J.Z. (1985) Numerical modelling of oxygen cycling in the upper ocean. WHOI SSF Report No. 42 (unpublished). 50 pp.

Coale, K.H. \& K.W. Bruland (1987) Oceanic stratified euphotic zone as elucidated by ${ }^{234} \mathrm{Th}:{ }^{238} \mathrm{Th}$ disequilibria. Limn. \& Ocean. 32, 189-200.

Coantic, Michel (1986) A model of gas transfer across air-water interfaces with capillary waves. J. Geophys. Res. 91, 3925-3943.

Craig, H. \& T. Hayward (1987) Oxygen supersaturation in the ocean: biological vs. physical contributions. Science 235, 199-202.

Craig, H. \& R.F. Weiss (1968) Argon concentrations in the ocean: a discussion. Earth \& Plan. Sci. Lett. 5, 175-183.

Craig, H. \& R.F. Weiss (1971) dissolved gas saturation anomalies and excess helium in the ocean. Earth E Plan. Sci. Lett. 10, 289.

Crawford, G.B. \& D.M. Farmer (1987) On the spatial distribution of ocean bubbles. J. Geophys. Res. 92, 8231-8243.

Dawson, Peter H. ed. (1978) Quadropole Mass Spectrometry. Elsevier Sci. Publ. Co., N.Y. $350 \mathrm{pp}$.

Deacon, E.L. (1977) Gas transfer to and across an air-water interface. Tellus 16, 21.

Deuser, W.G. (1986) Seasonal and interannual variations in deep- water particle flux in the Sargasso Sea and their relation to surface hydrography. Deep Sea Res. 33, 225-246.

Deuser, W.G. et al. (1988) Temporal variations of particle fluxes in the deep subtropical and tropical North Atlantic: Eulerian versus Lagrangian effects. J. Geophys. Res. 93, 6857-6862. 
Deuser, W.G. et al. (1981) Seasonality in the supply of sediment to the deep Sargasso Sea and implications for the rapid transfer of matter to the deep ocean. Deep Sea Res. 28A, 495-505.

Druffel, E.R.M. (1989) Decade time scale variability of ventilation in the North Atlantic: high precision measurements of bomb radiocarbon in banded corals. J. Geophys. Res. 94, 3271-3285.

Dugdale, R.C. \& J.J. Goering (1967) Uptake of new and regenerated forms of nitrogen in primary productivity. Limn. \& Ocean. 12, 196-206.

Eppley, R.W. \& B.J. Peterson (1979) [E\&P] Particulate organic matter flux and planktonic new production in the deep ocean. Nature 282, 677-680.

Emerson, S. (1988) Seasonal oxygen cycles and biological new production in surface waters of the subartic Pacific Ocean. J. Geophys. Res. 92, 6535-6544.

Fitzwater et al. (1982) Metal contamination and its effects on primary production measurements. Limnol. Oceanogr. 27, 544-551.

Friehe, C. \& S.E. Pazan (1978) Performance of an air-sea interaction buoy. J. Appl. Met. 17, 1488-1497.

Fuchs, G., W. Roether \& P. Schlosser (1986) Excess ${ }^{3} \mathrm{He}$ in the surface layer. J. Geophys. Res. 92, 6559-6568.

Frankignoul, C. (1981) Low-frequency temperature fluctuations off Bermuda. J. Geophys. Res. 86, 6522-6528.

Frost, B.W. (1984) Utilization of phytoplankton production in the surface layer. In: Global Ocean Flux Study: Proceedings of a workshop, September 10-14, 1984. National Academy Press, Washington D.C., pp. 125-135.

Garrett, C. (1979) Mixing in the ocean interior. Dyn. Atmos. Oceans 3, 239-265.

Gargett, A.E. (1984) Vertical eddy diffusivity in the ocean interior. J. Mar. Res. 42, 359-393.

Gieskes et al. (1979) Current ${ }^{14} \mathrm{C}$ methods for measuring primary production: gross underestimates in oceanic waters. Neth. J. of Sea Res. 13, 58-78.

Goering, J.J. et al. (1970) Nitrogen uptake by phytoplankton in the discontinuity layer of the eastern subtropical Pacific Ocean. Limn. E Ocean. 15, 789-796.

Goldman, J.C. (1988) Spatial and temporal discontinuities of biological processes in pelagic surface waters. In: Toward a theory on biological and physical interactions in the world ocean (ed. B. J. Rothschild). D. Reidel, Norwell, Mass., 273-296.

Goldman, J.C., M.R. Dennett \& N.M. Frew (1988) Surfactant effects on air-sea gas exchange. Deep Sea Res. 35, 1953-1970. 
Goldman, J.C. \& M.R. Dennett (1985) Susceptibility of some marine phytoplankton to cell breakage during filtration and post-filtration rinsing. J. Exp. Mar. Biol. Ecol..

Hamilton, J.M., M.R. Lewis \& B.R. Ruddick (1989) Vertical fluxes of nitrate associated with salt fingers in the world's oceans. J. Geophys. Res. 94, 2137-2145.

Harris, G.P. (1980) The measurement of photosynthesis in natural populations of phytoplankton. In: Primary Productivity in the Sea (ed. P.G. Falkowski). Plenum Press, N.Y. 531 pp.

Hogg, N.G. et al. (1978) Eddies, islands, and mixing. J. Geophys. Res. 83, 2921-2938.

Isemer, H.J. \& L. Hasse (1985) The Bunker Climate Atlas of the North Atlantic Ocean. (Springer-Verlag, Berlin). 218 pp.

Jahne, B., T. Wais, and M. Barabas (1984) A new optical bubble measuring device: a simple model for bubble contribution to gas exchange. In: Gas Transfer at Water Surfaces (W. Brutsaert and G.H. Jirka, eds., Reidel Publ. Co.), 237-246.

Jahne et al. (1987) Measurements of the diffusion coefficients of sparingly soluble gases in water. J. Geophys. Res. 92, 10767-1076.

Jenkins, W.J. (1977) Tritium-helium dating in the Sargasso Sea: a measurement of oxygen utilization rates. Science 196, 291-292.

Jenkins, W.J. (1980) Tritium and ${ }^{3} \mathrm{He}$ in the Sargasso Sea. J. Mar. Res. 38, 533-569.

Jenkins, W.J. (1982a) Oxygen utilization rates in the N. Atlantic subtropical gyre and primary production in oligotrophic systems. Nature 300, 246-248.

Jenkins, W.J. (1982b) On the climate of a subtropical ocean gyre: decade time-scale variations in water mass renewal in the Sargasso Sea. J. Mar. Res. 40 (Supp), 265-290.

Jenkins, W.J. (1987) ${ }^{3} \mathrm{H}$ and ${ }^{3} \mathrm{He}$ in the Beta Triangle: observations of ventilation and oxygen utilization rates. J. Phys. Ocean. 17, 763-783.

Jenkins, W.J. (1988a) Nitrate flux into the euphotic zone near Bermuda. Nature 331, 521-523.

Jenkins, W.J. (1988b) The use of anthropogenic tritium and helium-3 to study subtropical gyre ventilation and circulation. Phil. Trans. R. Soc. 325, 43-61.

Jenkins, W.J. \& W.B. Clarke (1976) The distribution of ${ }^{3} \mathrm{He}$ in the western Atlantic Ocean. Deep Sea Res. 23, 481-494.

Jenkins, W.J. \& J. Goldman (1985) [J\&G] Seasonal oxygen cycling and primary production in the Sargasso Sea. J. Mar. Res. 43, 465-491.

Johnson, K.S. et al. (1979) Biological production and the exchange of oxygen and carbon dioxide across the sea surface in Stuart Channel, British Columbia. Limn. ES Ocean. 24, 474-482. 
Joyce T.M. (1988) On regulation of primary production by physical processes in the ocean: two case studies. In: Toward a theory on biological and physical interactions in the world ocean (ed. B. J. Rothschild). D. Reidel, Norwell, Mass., 39-50.

Karl, D.M. \& G.A. Knauer (1984) Vertical distribution and exchange of organic matter in the northeast Pacific Ocean: evidence for multiple zones of biological activity. Deep Sea Res. 31, 221-243.

Kawase, M. \& J.L. Sarmiento (1985) Nutrients in the Atlantic thermocline. J. Geophys. Res. 90, 8961-8979.

Kester, D. (1975) Dissolved gases other than carbon dioxide. In: Chemical Oceanography, Vol. V1 (J.P. Riley \& G. Skirrow eds.). Academic Press, N.Y., pp. 497-557.

Klein, P. \& B. Coste (1984) Effects of wind-stress variability on nutrient transport in the mixed layer. Deep Sea Res. 31, 21-37.

Knap, A.H. et al. (1986) Significance of atmospheric-derived fixed nitrogen on productivity of the Sargasso Sea. Nature 320, 158-160.

Knauer, G.A. et al. (1984) The flux of particulate organic matter out of the euphotic zone. In: Global ocean flux study, proceedings of a workshop. National Academy Press.

Large, W.G. et al. (1985) Upper ocean thermal response to fall forcing in the northeast Pacific. J. Geophys. Res. 16, 1524-1550.

Laws, E.A. et al. (1987) High phytoplankton growth and production rates in the North Pacific subtropical gyre. Limnol. Oceanogr. 34, 904-918.

Leetma, A. \& A.F. Bunker (1978) Updated charts of the mean annual wind stress, convergences in the Ekman layers and Sverdrup transports in the North Atlantic. J. Mar. Res. 36, 311-322.

Levitus, S. (1982) Climatological Atlas of the World Ocean. NOAA Prof. Paper 13, USGPO, Wash., D.C. 173pp.

Lewis, J.M. \& T.H. Grayson (1972) The adjustment of surface wind and pressure by Sasaki's variational matching technique. J. Appl. Met. 11, 586-597.

Lewis, M.R. et al. (1986) Vertical nitrate fluxes in the oligotrophic ocean. Science 234, 870-873.

Li, W.K.W. (1987) Experimental approaches to field measurements: methods and interpretation. In: Photsynthetic picoplankton (T. Platt \& W.K.W., eds.), Can. Bull. of Fish. \& Aquatic Sci. 214, 251-286.

Liss, P.S. (1983) Gas transfer, experiments, and geochemical implications. In: Air-sea exchange of gases and particles (P.S. Liss \& G.N. Slinn, eds.), D. Reidel Publ. Co., Dordrecht, pp. 241-298. 
Liss, P.S. \& L. Merlivat (1986) Air-sea gas exchange rates: introduction and synthesis. In: The role of air-sea exchange in geochemicaly cycling (Adv. Sci. Inst. Ser., P. Buat-Menard, ed.), D. Reidel, Hingham, MA, 113-127.

Lomb, N.R. (1976) Least-squares frequency analysis of unequally spaced data. Astrophys. and Space Sci. 39, 447-462.

Lott, D.E. \& W.J. Jenkins (1984) An automated cryogenic charcoal trap system for helium isotope mass spectrometry. Rev. Sci. Instrum. 55, 1982-1988.

Marra, J. \& K.R. Heinemann (1984) A comparison between non-contaminating and conventional incubation procedures in primary production measurements. Limnol. Oceanogr. 29, 389-392.

Martin et al. (1987) VERTEX: Carbon cycling in the northeast Pacific. Deep Sea Res. 32, 267-286.

Martinez, L.M. et al. (1983) Nitrogen fixation by floating diatom mats: a source of new nitrogen to oligotrophic ocean waters. Science 221, 152-154.

McCarthy, J.J. (1984) Measuring oceanic primary production. In: Global ocean flux study, proceedings of a workshop. National Academy Press.

McCarthy, J.J. \& E.J. Carpenter (1983) Nitrogen cycling in near-surface waters of the open ocean. In: Nitrogen in the Marine Environment (ed. E.J. Carpenter \& D.G. Capone). Academic Press, NY, 900 pp.

Menzel, D.W. \& J.H. Ryther (1960) The annual cycle of primary production in the Sargasso Sea off Bermuda. Deep Sea Res. 6, 351-367.

Menzel, D.W. \& J.H. Ryther (1961) Annual variations in primary production of the Sargasso Sea off Bermudal. Deep Sea Res. 7, 282-288.

Merlivat \& Memery (1983) Gas exchange across an air-water interface: experimental results and modelling of bubble contribution to transfer. J. Geophys. Res. 88, 707-724.

Michaels, A.F. \& M.W. Silver (1988) Primary production, sinking fluxes and the microbial food web. Deep Sea Res. 35, 473-490.

Musgrave, D.L., J. Chou and W.J. Jenkins (1988) Application of a model of upper ocean physics for studying seasonal cycles of oxygen. J. Geophys. Res. 93, 15679-15700.

Nelson, D.M. et al. (1989) Enhanced near-surface nutrient availability and new production resulting from the frictional decay of a Gulf Stream warm-core ring. In press in Deep Sea Res.

Okubo, A. (1971) Oceanic diffusion diagrams. Deep Sea Res. 18, 789-802.

Packard, T.T. et al. (1975) The effect of temperature on the respiratory electron transport system in marine plankton. Deep Sea Res. 24, 237-249. 
Paulson, C.A. \& J.J. Simpson (1977) Irradiance measurements in the upper ocean. J. Phys. Oceanogr. 7, 952-956.

Peng, T.H. et al. (1974) Surface radon measurements in the North Pacific at ocean station Papa. J. Geophys. Res. 79, 1772-1780.

Peterson, B.J. (1980) Aquatic primary productivity and the ${ }^{14} \mathrm{C}-\mathrm{CO}_{2}$ method: a history of the productivity problem. Ann. Rev. System. Ecol. 11, 359-385.

Platt, T. (1984a) Primary production in the central North Pacific: comparison of oxygen and carbon fluxes. Deep Sea Res. 31, 1311-1319.

Platt, T. \& W.G. Harrison (1985) Biogenic fluxes of carbon and oxygen in the ocean. Nature 318, 55-58.

Platt, T. \& W.G. Harrison (1986) Reconciliation of carbon and oxygen fluxes in the upper ocean. Deep Sea Res. 33, 273-276.

Platt, T. \& D.V. Subba Rao (1975) In: Photosynthesis and Productivity in Different Environments. Internat. Biological Programme, Cambridge Univ. Press. p. 249.

Press, W.H., B.P. Flannery, S.A. Teukolsky and W.T. Vetterling (1986) Numerical Recipes (Cambridge University Press, NY). 818 pp.

Price, J.F., R.A. Weller and R. Pinkel (1986) Diurnal cycling: observations and models of upper ocean response to diurnal heating, cooling and wind mixing. J. Geophys. Res. 91, 8411-8427.

Reid, J.L. \& E. Schulenberger (1986) Oxygen saturation and carbon uptake near $28^{\circ} \mathrm{N}, 155^{\circ} \mathrm{W}$. Deep Sea Res. 33, 267-271.

Richardson, P.L. (1983) Gulf Stream Rings. In Eddies in Marine Science (ed. A.R. Robinson, Springer-Verlag, NY), pp 19-43.

Riley, G.A. (1951) Oxygen, phosphate, and nitrate in the Atlantic Ocean. Bull. Bingham Oceanogr. Coll. 13, 1-126.

Roether, W. (1985) Field measurements of gas exchange. In: Dynamic Processes in the Chemistry of the Upper Ocean. Plenum Press, NY.

Sano, Y., T. Tominaga and H. Wakita (1982) Elemental and isotopic abundances of rare gases in natural gases obtained by a quadropole mass spectrometer. Geochem. Journal 16, 279-286.

Sarmiento, J.L. (1983) A simulation of bomb tritium entry into the Atlantic Ocean. J. Phys. Ocean. 13, 1924-1939.

Scargle, J.D. (1982) Studies in astronomical time series analysis, II: statistical aspects of spectral analysis of unevenly spaced data. Astrophys. J., 263, 835-853. 
Schulenberger, E. \& J.L. Reid (1981) The Pacific shallow oxygen maximum, deep chlorophyll maximum and primary productivity, reconsidered. Deep Sea Res. 28A, 901-919.

Schmitt (1981) The form of the temperature salinity relationship in the Central Water: evidence for double-diffusive mixing. J. Phys. Oceanogr. 11, 1015-1026.

Sharp, J.H. et al. (1980) Phytoplankton rate processes in the oligotrophic waters of the central North Pacific Ocean. J. Plankton Res. 2, 335-353.

Simonot, J. \& H. Le Treut (1986) A climatological field of mean optical properties of the world ocean. J. Geophys. Res. 91, 6642-6646.

Smith, S.D. \& E.P. Jones (1985) Evidence for wind-pumping of air-sea exchange based on direct measurements of $\mathrm{CO}_{2}$ fluxes. J. Geophys. Res. 90, 869-875.

Spitzer, W.S. \& W.J. Jenkins (1986) Argon as an abiogenic analog of oxygen in upper ocean seasonal gas cycles. Eos Trans. Amer. Geophys. Union 67, 1018.

Spitzer, W.S. \& W.J. Jenkins (1987) The seasonal cycle of argon in the upper Sargasso Sea. Eos Trans. Amer. Geophys. Union 68, 1700.

Spitzer, W.S. \& W.J. Jenkins. (1989) Rates of vertical mixing, gas exchange, and new production: estimates from seasonal gas cycles in the upper ocean near Bermuda. J. Mar. Res. 47, 169-196.

Stern, M. (1987) Horizontal entrainment and detrainment in large-scale eddies. J. Phys. Ocean. 17, 1688-1695.

Stommel, H. et al. (1969) Observations of the diurnal thermocline. Deep Sea Res. 16 (Supp), 269-284.

Takahashi, T. et al (1985) Redfield ratio based on chemical data from isopycnal surfaces. $J$. Geophys. Res. 90, 6907-6924.

Talley, L.D. \& M.E. Raymer (1982) Eighteen degree water variability. J. Mar. Res. 40 (Supp), 757-775.

Thomas et al. (1989) Modelling the seasonal cycle of dissolved $\mathrm{O}_{2}$ in the upper ocean at Ocean Weather Station P. Submitted to J. Geophys. Res.

Thorpe, S.A. (1984a) The effect of bubbles produced by breaking wind-waves on gas flux across the sea-surface. Ann. Geophys. 2, 53-56.

Thorpe, S.A. (1984c) The role of bubble produced by breaking waves in super-saturating the near-surface ocean mixing layer with oxygen. Annales Geophysicae 2, 53-56.

Thorpe, S.A. (1986) Measurements with an automatically recording inverted echo sounder; ARIES and the bubble clouds. J. Phys. Ocean. 16, 1462-1478.

Tijssen, B. (1979) Diurnal oxygen rhythm and primary production in the mixed layer of the Atlantic Ocean at $20^{\circ} \mathrm{N}$. Netherlands J. of Sea Res. 13, 79-84. 
Tsunogai, S. \& N. Tanaka (1980) Flux of oxygen across the air- sea interface as determined by the analysis of dissolved components in seawater. Geochem. J. 14, 227-234.

Weast, R.C. ed. (1984) CRC Handbook of Chemistry and Physics 65th ed. CRC Press.

Wesley, M.L. et al. (1982) Air-sea exchange of $\mathrm{CO}_{2}$ and evidence for enhanced upward fluxes. J. Geophys. Res. 87, 88276-8832.

Weiss, R.F. (1968) Piggyback sampler for dissolved gas studies on sealed water samples. Deep Sea Res. 15, 695-699.

Weiss, R.F. (1970) The solubility of nitrogen, oxygen and argon in water and sea water. Deep Sea Res. 17, 721-735.

Weiss, R.F. (1971) The effect of salinity on the solubility of argon in seawater. Deep Sea Res. $18,225-230$.

Weiss, R.F. (1971) Solubility of helium and neon in water and seawater. J. Chem. Eng. Data 16, 235-241.

Weiss, R.F. \& H. Craig (1973) Precise shipboard determination of dissolved nitrogen, oxygen, argon and total inorganic carbon by gas chromatography. Deep Sea Res. 20, 291-303.

Weiss, R.F. \& T.K. Kyser (1978) Solubility of krypton in water and seawater. J. Chem. Eng. Data 23, 69-72.

WHOI \& BBSR (1988) Station "S" off Bermuda: physical measurements 1954-1984. WHOI Contrib. No. 6894, BBSR Spec. Publ. No. 29.

Williams, P.J. LeB. et al. (1979) Agreement between the ${ }^{14} \mathrm{C}$ and $\mathrm{O}_{2}$ methods of measuring phytoplankton production: reassessment of the photosynthetic quotient. Ocean. Acta 2, 411-416.

Williams, P.J. LeB. et al. (1983) Comparisons of ${ }^{14} \mathrm{C}$ and $\mathrm{O}_{2}$ measurements of phytoplankton production in oligotrophic waters. Nature 305, 49-50.

Wise, D.L. \& G. Houghton (1966) The diffusion coefficients of ten slightly soluble gases in water at $10-60^{\circ}$ C. Chem. Engr. Sci. 21, 999-1010.

Wise, D.L. \& G. Houghton (1968) Diffusion coeffficients of neon, krypton, xenon, carbon monoxide and nitric oxide in water at $10-60^{\circ} \mathrm{C}$. Chem. Engr. Sci. 23, 1211-1216.

Woods, J.D. \& W. Barkmann (1986) A lagrangian mixed layer model of Atlantic $18^{\circ} \mathrm{C}$ water formation. Nature 319, 574-576.

Worthington, L.V. (1976) On the North Atlantic circulation. The Johns Hopkins Oceanographic Studies, vol. 6 Johns Hopkins Univ. Press, Baltimore, 110 pp.

Worthington, L.V. (1977) Intensification of the Gulf Stream after the winter of 1976-1977. Nature 270, 415-417.

Wunsch, C. (1983) Western North Atlantic Interior. In Eddies in Marine Science (ed. A.R. Robinson, Springer-Verlag, NY), pp 46-65. 


\section{Appendix A}

\section{Measured Argon Concentrations}

\begin{tabular}{rrrrrrrr} 
Sta. Year & \multicolumn{1}{c}{ Bt. Depth } & Temp. Argon & Argon & Oxygen \\
no. & time & no. & (m) & (C) & Conc. & Supsatn & Supsatn \\
\hline 562 & 85.25 & 1 & 1 & 19.07 & 2.541 & 2.3 & 4.8 \\
562 & 85.25 & 27 & 1 & 19.04 & 2.494 & 0.3 & 4.8 \\
562 & 85.25 & 28 & 10 & 18.86 & 2.513 & 0.7 & 5.6 \\
562 & 85.25 & 29 & 20 & 18.72 & 2.546 & 1.8 & 4.9 \\
562 & 85.25 & 3 & 25 & 18.79 & 2.505 & 0.3 & 4.3 \\
562 & 85.25 & 30 & 30 & 18.86 & 2.538 & 1.7 & 4.0 \\
562 & 85.25 & 31 & 41 & 18.70 & 2.528 & 1.0 & 2.0 \\
562 & 85.25 & 4 & 50 & 18.82 & 2.572 & 3.0 & 2.2 \\
562 & 85.25 & 33 & 60 & 18.69 & 2.550 & 1.9 & -0.7 \\
562 & 85.25 & 34 & 70 & 18.65 & 2.512 & 0.3 & 0.7 \\
562 & 85.25 & 35 & 80 & 18.65 & 2.508 & 0.1 & 0.7 \\
562 & 85.25 & 37 & 125 & 18.64 & 2.511 & 0.2 & 0.7 \\
562 & 85.25 & 38 & 173 & 18.61 & 2.503 & -0.2 & 0.3 \\
562 & 85.25 & 38 & 173 & 18.61 & 2.536 & 1.1 & 0.3 \\
562 & 85.25 & 8 & 190 & 18.64 & 2.516 & 0.3 & 0.7 \\
562 & 85.25 & 9 & 239 & 18.59 & 2.517 & 0.3 & 0.6 \\
562 & 85.25 & 10 & 288 & 18.60 & 2.533 & 1.0 & 0.6 \\
562 & 85.25 & 11 & 336 & 18.16 & 2.571 & 1.6 & -9.0 \\
562 & 85.25 & 12 & 386 & 18.03 & 2.487 & -2.0 & -11.5 \\
562 & 85.25 & 12 & 386 & 18.03 & 2.557 & 0.7 & -11.5 \\
562 & 85.25 & 24 & 2188 & 3.60 & 3.484 & -0.2 & -15.3 \\
564 & 85.32 & 27 & 1 & 20.33 & 2.514 & 3.5 & 5.7 \\
564 & 85.32 & 28 & 5 & 19.51 & 2.532 & 2.7 & 5.7 \\
564 & 85.32 & 3 & 25 & 19.43 & 2.542 & 2.9 & 6.5
\end{tabular}




$\begin{array}{rrrrrrrr}564 & 85.32 & 31 & 43 & 19.20 & 2.512 & 1.3 & 5.1 \\ 564 & 85.32 & 33 & 61 & 18.88 & 2.544 & 2.0 & 3.3 \\ 564 & 85.32 & 34 & 68 & 18.81 & 2.514 & 0.7 & 0.1 \\ 564 & 85.32 & 36 & 89 & 18.82 & 2.548 & 2.0 & -0.5 \\ 564 & 85.32 & 37 & 127 & 18.57 & 2.534 & 1.0 & -1.1 \\ 564 & 85.32 & 38 & 167 & 18.46 & 2.572 & --- & --- \\ 564 & 85.32 & 38 & 167 & 18.46 & 2.553 & --- & --- \\ 564 & 85.32 & 24 & 2207 & 3.50 & 3.694 & 5.5 & -16.6 \\ 566 & 85.41 & 27 & 1 & 22.97 & 2.405 & 3.6 & 5.6 \\ 566 & 85.41 & 28 & 5 & 22.37 & 2.394 & 2.0 & 4.5 \\ 566 & 85.41 & 2 & 10 & 22.04 & 2.421 & 2.6 & 7.8 \\ 566 & 85.41 & 29 & 10 & 22.06 & 2.411 & 2.3 & 7.4 \\ 566 & 85.41 & 30 & 20 & 21.95 & 2.404 & 1.7 & 8.4 \\ 566 & 85.41 & 31 & 30 & 21.20 & 2.521 & 5.3 & 8.4 \\ 566 & 85.41 & 32 & 40 & 20.45 & 2.468 & 1.8 & 7.7 \\ 566 & 85.41 & 33 & 50 & 19.78 & 2.522 & 2.8 & 9.1 \\ 566 & 85.41 & 34 & 60 & 19.24 & 2.512 & 1.3 & 9.2 \\ 566 & 85.41 & 35 & 70 & 18.82 & 2.521 & 0.9 & 1.2 \\ 566 & 85.41 & 36 & 81 & 19.02 & 2.525 & 1.4 & 4.7 \\ 566 & 85.41 & 37 & 91 & 18.61 & 2.502 & -0.2 & -1.3 \\ 566 & 85.41 & 39 & 176 & 18.23 & 2.554 & 1.1 & -1.4 \\ 566 & 85.41 & 24 & 2178 & 3.57 & 3.579 & 2.5 & -18.5 \\ 567 & 85.49 & 1 & 1 & 26.13 & 2.309 & 4.9 & 9.2 \\ 567 & 85.49 & 28 & 5 & 25.73 & 2.265 & 2.2 & 6.9 \\ 567 & 85.49 & 29 & 9 & 25.66 & 2.294 & 3.4 & 8.3 \\ 567 & 85.49 & 30 & 18 & 23.96 & 2.353 & 3.2 & 9.6 \\ 567 & 85.49 & 32 & 33 & 21.39 & 2.476 & 3.9 & 7.6 \\ 567 & 85.49 & 33 & 40 & 20.69 & 2.487 & 3.0 & 9.8 \\ 567 & 85.49 & 34 & 46 & 20.31 & 2.526 & 4.0 & 6.7 \\ 567 & 85.49 & 35 & 55 & 19.92 & 2.529 & 3.3 & 7.8 \\ 567 & 85.49 & 36 & 66 & 19.91 & 2.504 & 2.3 & 4.7 \\ 567 & 85.49 & 37 & 76 & 19.32 & 2.507 & 1.3 & 1.8 \\ 567 & 85.49 & 39 & 179 & 18.50 & 2.517 & 0.2 & -5.8 \\ 567 & 85.49 & 39 & 179 & 18.50 & 2.561 & 1.9 & -5.8 \\ 567 & 85.49 & 24 & 2156 & 3.61 & 3.449 & -1.2 & -18.3 \\ 569 & 85.56 & 27 & 1 & 27.25 & 2.229 & 3.1 & 24.0 \\ 569 & 85.56 & 28 & 5 & 26.94 & 2.227 & 2.5 & 17.2 \\ 569 & 85.56 & 29 & 10 & 26.65 & 2.224 & 1.8 & 12.9 \\ 569 & 85.56 & 30 & 20 & 26.47 & 2.303 & 5.1 & 19.0 \\ 569 & 85.56 & 31 & 30 & 25.59 & 2.292 & 3.1 & 15.9 \\ 569 & 85.56 & 32 & 39 & 22.11 & 2.478 & 5.2 & 18.7 \\ 569 & 85.56 & 33 & 49 & 21.06 & 2.474 & 3.2 & 15.1 \\ 569 & 85.56 & 34 & 59 & 20.87 & 2.500 & 3.9 & 14.7 \\ 569 & 85.56 & 35 & 68 & 20.06 & 2.488 & 1.9 & 16.7 \\ 569 & 85.56 & 36 & 77 & 19.56 & 2.501 & 1.5 & 11.2 \\ & & & & & & & \end{array}$




$\begin{array}{rrrrrrrr}569 & 85.56 & 38 & 119 & 18.90 & 2.503 & 0.4 & 1.8 \\ 569 & 85.56 & 39 & 177 & 18.59 & 2.523 & 0.6 & 0.4 \\ 569 & 85.56 & 39 & 177 & 18.59 & 2.582 & 3.0 & 0.4 \\ 569 & 85.56 & 24 & 2152 & 3.61 & 3.450 & -1.1 & -14.5 \\ 571 & 85.65 & 1 & 1 & 28.31 & 2.227 & 4.7 & 26.0 \\ 571 & 85.65 & 28 & 5 & 27.62 & 2.157 & 0.3 & 18.1 \\ 571 & 85.65 & 29 & 8 & 27.46 & 2.177 & 1.0 & 11.4 \\ 571 & 85.65 & 2 & 10 & 27.41 & 2.188 & 1.4 & 20.1 \\ 571 & 85.65 & 30 & 13 & 27.53 & 2.205 & 2.5 & 19.5 \\ 571 & 85.65 & 3 & 24 & 27.25 & 2.192 & 1.4 & 18.7 \\ 571 & 85.65 & 32 & 30 & 26.37 & 2.242 & 2.3 & 17.7 \\ 571 & 85.65 & 33 & 39 & 22.88 & 2.451 & 5.5 & 21.0 \\ 571 & 85.65 & 34 & 48 & 21.67 & 2.467 & 4.0 & 16.0 \\ 571 & 85.65 & 35 & 67 & 20.42 & 2.478 & 2.2 & 13.4 \\ 571 & 85.65 & 5 & 68 & 19.80 & 2.499 & 1.9 & 13.2 \\ 571 & 85.65 & 37 & 86 & 19.47 & 2.498 & 1.2 & 6.9 \\ 571 & 85.65 & 6 & 92 & 19.16 & 2.504 & 0.9 & -0.4 \\ 571 & 85.65 & 38 & 121 & 18.92 & 2.500 & 0.3 & 0.3 \\ 571 & 85.65 & 39 & 182 & 18.41 & 2.518 & 0.1 & 1.6 \\ 571 & 85.65 & 24 & 2174 & 3.61 & 3.486 & -0.1 & -15.6 \\ 571 & 85.65 & 25 & 2369 & 3.63 & 3.473 & -0.5 & -11.4 \\ 574 & 85.79 & 27 & 1 & 25.98 & 2.260 & 2.3 & 13.1 \\ 574 & 85.79 & 28 & 5 & 25.68 & 2.272 & 2.3 & 17.5 \\ 574 & 85.79 & 29 & 10 & 25.69 & 2.256 & 1.7 & 12.6 \\ 574 & 85.79 & 30 & 20 & 25.71 & 2.253 & 1.6 & 11.1 \\ 574 & 85.79 & 32 & 48 & 25.65 & 2.252 & 1.4 & 9.1 \\ 574 & 85.79 & 33 & 51 & 25.64 & 2.232 & 0.5 & 12.7 \\ 574 & 85.79 & 35 & 69 & 25.11 & 2.257 & 0.7 & 11.9 \\ 574 & 85.79 & 36 & 80 & ----- & 2.327 & --- & --- \\ 574 & 85.79 & 37 & 88 & 23.28 & 2.345 & 1.6 & 4.2 \\ 574 & 85.79 & 38 & 123 & 21.66 & 2.402 & 1.3 & -1.3 \\ 574 & 85.79 & 39 & 165 & 19.63 & 2.474 & 0.6 & -5.5 \\ 574 & 85.79 & 24 & 2211 & 3.80 & 3.468 & -0.2 & -13.0 \\ 577 & 85.85 & 27 & 3 & 25.04 & 2.280 & 1.7 & 7.4 \\ 577 & 85.85 & 28 & 5 & 24.92 & 2.261 & 0.6 & 5.4 \\ 577 & 85.85 & 29 & 10 & 24.89 & 2.330 & 3.6 & --- \\ 577 & 85.85 & 32 & 42 & 24.89 & 2.246 & -0.1 & 6.4 \\ 577 & 85.85 & 33 & 53 & 24.89 & 2.275 & 1.2 & 5.4 \\ 577 & 85.85 & 34 & 63 & 24.90 & 2.244 & -0.2 & 4.5 \\ 577 & 85.85 & 35 & 74 & 24.88 & 2.242 & -0.3 & 1.9 \\ 577 & 85.85 & 36 & 84 & 24.89 & 2.359 & 4.9 & 8.8 \\ 577 & 85.85 & 37 & 94 & 24.77 & 2.257 & 0.2 & -14.4 \\ 577 & 85.85 & 24 & 2222 & 3.56 & 3.441 & -1.7 & -16.7 \\ 580 & 85.93 & 27 & 1 & 22.83 & 2.346 & 0.9 & --- \\ 580 & 85.93 & 28 & 5 & 22.80 & 2.448 & 5.2 & ---\end{array}$




$\begin{array}{rrrrrrrr}580 & 85.93 & 29 & 10 & 22.78 & 2.336 & 0.4 & --- \\ 580 & 85.93 & 31 & 32 & 22.78 & 2.334 & 0.3 & --- \\ 580 & 85.93 & 32 & 42 & 22.74 & 2.334 & 0.2 & --- \\ 580 & 85.93 & 33 & 55 & 22.73 & 2.348 & 0.8 & --- \\ 580 & 85.93 & 34 & 69 & 22.71 & 2.377 & 2.0 & --- \\ 580 & 85.93 & 35 & 72 & 22.65 & 2.335 & 0.1 & --- \\ 580 & 85.93 & 37 & 91 & 22.63 & 2.334 & 0.0 & --- \\ 580 & 85.93 & 38 & 127 & 21.44 & 2.414 & 1.4 & --- \\ 580 & 85.93 & 39 & 173 & 19.69 & 2.498 & 1.7 & --- \\ 580 & 85.93 & 24 & 2222 & 3.50 & 3.466 & -1.0 & --- \\ 584 & 86.09 & 27 & 1 & 20.29 & 2.433 & 0.1 & 16.5 \\ 584 & 86.09 & 28 & 5 & 20.28 & 2.425 & -0.2 & 14.1 \\ 584 & 86.09 & 29 & 10 & 20.26 & 2.432 & 0.0 & 15.8 \\ 584 & 86.09 & 30 & 20 & 20.27 & 2.420 & -0.5 & 13.5 \\ 584 & 86.09 & 31 & 31 & 20.38 & 2.444 & 0.7 & 14.7 \\ 584 & 86.09 & 32 & 41 & 20.23 & 2.428 & -0.2 & 20.8 \\ 584 & 86.09 & 33 & 52 & 20.25 & 2.415 & -0.7 & 12.8 \\ 584 & 86.09 & 34 & 63 & 20.22 & 2.429 & -0.2 & 17.7 \\ 584 & 86.09 & 35 & 74 & 20.26 & 2.436 & 0.1 & 16.4 \\ 584 & 86.09 & 37 & 95 & 19.76 & 2.475 & 0.8 & 6.9 \\ 584 & 86.09 & 38 & 131 & 19.16 & 2.492 & 0.4 & 5.2 \\ 584 & 86.09 & 39 & 174 & 18.52 & 2.514 & 0.1 & 4.8 \\ 584 & 86.09 & 39 & 174 & 18.52 & 2.511 & 0.0 & 4.8 \\ 584 & 86.09 & 24 & 2200 & 3.66 & 3.448 & -1.1 & -6.2 \\ 585 & 86.26 & 27 & 1 & ----- & 2.463 & --- & --- \\ 585 & 86.26 & 28 & 5 & 19.87 & 2.474 & 0.9 & -4.6 \\ 585 & 86.26 & 2 & 10 & 19.72 & 2.479 & 0.9 & 14.0 \\ 585 & 86.26 & 29 & 10 & 19.81 & 2.489 & 1.5 & 4.3 \\ 585 & 86.26 & 30 & 20 & 19.64 & 2.470 & 0.4 & 7.4 \\ 585 & 86.26 & 31 & 31 & 19.64 & 2.557 & 3.9 & 5.3 \\ 585 & 86.26 & 32 & 41 & 19.45 & 2.593 & 5.0 & 4.8 \\ 585 & 86.26 & 33 & 51 & 19.42 & 2.496 & 1.0 & -0.9 \\ 585 & 86.26 & 34 & 68 & 19.44 & 2.484 & 0.6 & 4.9 \\ 585 & 86.26 & 35 & 71 & 19.44 & 2.480 & 0.4 & 6.9 \\ 585 & 86.26 & 37 & 91 & 19.27 & 2.472 & -0.2 & 12.7 \\ 585 & 86.26 & 38 & 125 & 19.19 & 2.560 & 3.2 & 10.8 \\ 585 & 86.26 & 39 & 177 & 19.07 & 2.482 & -0.1 & 3.7 \\ 585 & 86.26 & 39 & 177 & 19.07 & 2.511 & 1.0 & 3.7 \\ 585 & 86.26 & 26 & 2467 & 3.31 & 3.479 & -1.1 & -17.6 \\ 587 & 86.33 & 27 & 1 & 20.08 & 2.461 & 0.8 & 1.3 \\ 587 & 86.33 & 1 & 3 & 20.00 & 2.453 & 0.4 & 3.5 \\ 587 & 86.33 & 28 & 5 & 20.50 & 2.472 & 2.0 & 3.1 \\ 587 & 86.33 & 29 & 10 & 20.05 & 2.543 & 4.2 & 3.4 \\ 587 & 86.33 & 2 & 10 & 20.35 & 2.457 & 1.2 & 3.4 \\ 587 & 86.33 & 3 & 25 & 19.93 & 2.451 & 0.2 & 2.6\end{array}$




$\begin{array}{rrrrrrrr}587 & 86.33 & 31 & 30 & 19.92 & 2.444 & -0.2 & 2.0 \\ 587 & 86.33 & 32 & 40 & 19.90 & 2.464 & 0.6 & 2.0 \\ 587 & 86.33 & 33 & 50 & 19.88 & 2.463 & 0.5 & 1.9 \\ 587 & 86.33 & 34 & 60 & 19.91 & 2.461 & 0.5 & 2.6 \\ 587 & 86.33 & 34 & 60 & 19.91 & 2.504 & 2.3 & 2.6 \\ 587 & 86.33 & 35 & 70 & 19.94 & 2.468 & 0.9 & 2.6 \\ 587 & 86.33 & 36 & 80 & 19.82 & 2.477 & 1.1 & 3.0 \\ 587 & 86.33 & 37 & 90 & 19.78 & 2.462 & 0.4 & 2.9 \\ 587 & 86.33 & 38 & 127 & 19.42 & 2.484 & 0.7 & -0.4 \\ 587 & 86.33 & 39 & 170 & 18.91 & 2.486 & -0.2 & -6.4 \\ 587 & 86.33 & 24 & 2130 & 3.65 & 3.427 & -1.7 & -16.8 \\ 591 & 86.45 & 1 & 1 & 23.66 & 2.318 & 1.1 & 4.1 \\ 591 & 86.45 & 27 & 1 & 23.65 & 2.341 & 2.0 & 3.4 \\ 591 & 86.45 & 28 & 5 & 24.23 & 2.315 & 2.0 & 4.6 \\ 591 & 86.45 & 29 & 9 & 23.59 & 2.320 & 1.0 & 3.7 \\ 591 & 86.45 & 2 & 10 & 24.01 & 2.315 & 1.6 & 4.5 \\ 591 & 86.45 & 31 & 27 & 21.92 & 2.475 & 4.8 & 3.6 \\ 591 & 86.45 & 32 & 36 & 21.74 & 2.381 & 0.5 & 2.9 \\ 591 & 86.45 & 34 & 65 & 20.66 & 2.438 & 0.9 & 3.4 \\ 591 & 86.45 & 35 & 75 & 20.28 & 2.451 & 0.8 & 2.5 \\ 591 & 86.45 & 36 & 84 & 20.19 & 2.456 & 0.8 & 3.7 \\ 591 & 86.45 & 37 & 92 & 20.06 & 2.460 & 0.8 & 0.3 \\ 591 & 86.45 & 37 & 92 & 20.06 & 2.522 & 3.3 & 0.3 \\ 591 & 86.45 & 38 & 126 & 19.10 & 2.616 & 5.3 & -4.7 \\ 591 & 86.45 & 39 & 173 & 18.61 & 2.542 & 1.4 & -7.0 \\ 591 & 86.45 & 24 & 2163 & 3.63 & 3.459 & -0.9 & -18.0 \\ 593 & 86.52 & 27 & 1 & 26.29 & 2.244 & 2.1 & 4.8 \\ 593 & 86.52 & 28 & 5 & ----- & 2.170 & --- & --- \\ 593 & 86.52 & 29 & 11 & 25.82 & 2.259 & 2.0 & 4.4 \\ 593 & 86.52 & 30 & 21 & 25.07 & 2.282 & 1.8 & 4.4 \\ 593 & 86.52 & 3 & 25 & 22.21 & 2.387 & 1.5 & 6.5 \\ 593 & 86.52 & 31 & 32 & 22.04 & 2.406 & 2.0 & -4.0 \\ 593 & 86.52 & 31 & 32 & 22.04 & 2.297 & -2.6 & -4.0 \\ 593 & 86.52 & 32 & 42 & 21.14 & 2.422 & 1.1 & -4.9 \\ 593 & 86.52 & 33 & 52 & 20.55 & 2.429 & 0.3 & -5.7 \\ 593 & 86.52 & 34 & 62 & 20.07 & 2.466 & 1.0 & 1.5 \\ 593 & 86.52 & 35 & 72 & 19.36 & 2.484 & 0.5 & -1.2 \\ 593 & 86.52 & 5 & 76 & 19.26 & 2.490 & 0.5 & -0.2 \\ 593 & 86.52 & 37 & 92 & 19.02 & 2.494 & 0.2 & -4.1 \\ 593 & 86.52 & 7 & 155 & 18.54 & 2.544 & 1.3 & -3.3 \\ 593 & 86.52 & 39 & 169 & 18.51 & 2.562 & 2.0 & -1.6 \\ 593 & 86.52 & 39 & 169 & 18.51 & 2.526 & 0.5 & -1.6 \\ 593 & 86.52 & 8 & 208 & 18.54 & 2.547 & 1.4 & -1.6 \\ 593 & 86.52 & 9 & 262 & 18.42 & 2.543 & 1.0 & -2.0 \\ 593 & 86.52 & 10 & 308 & 18.30 & 2.544 & 0.8 & -2.3\end{array}$




$\begin{array}{rrrrrrrr}593 & 86.52 & 24 & 2171 & 3.69 & 3.478 & -0.2 & -18.0 \\ 597 & 86.66 & 27 & 1 & 27.99 & 2.168 & 1.4 & 8.7 \\ 597 & 86.66 & 1 & 1 & 27.90 & 2.169 & 1.3 & 11.0 \\ 597 & 86.66 & 2 & 10 & 27.74 & 2.178 & 1.5 & 8.0 \\ 597 & 86.66 & 29 & 10 & 27.73 & 2.219 & 3.4 & 10.3 \\ 597 & 86.66 & 2 & 10 & 27.74 & 2.174 & 1.3 & 8.0 \\ 597 & 86.66 & 31 & 32 & 25.77 & 2.296 & 3.5 & 13.4 \\ 597 & 86.66 & 4 & 50 & 22.10 & 2.418 & 2.7 & 15.0 \\ 597 & 86.66 & 33 & 53 & 21.88 & 2.433 & 2.9 & 16.2 \\ 597 & 86.66 & 34 & 61 & 21.27 & 2.434 & 1.8 & 10.5 \\ 597 & 86.66 & 5 & 77 & 20.36 & 2.476 & 1.9 & 6.9 \\ 597 & 86.66 & 36 & 84 & 20.32 & 2.476 & 1.9 & -4.1 \\ 597 & 86.66 & 6 & 105 & 19.27 & 2.500 & 0.9 & 0.9 \\ 597 & 86.66 & 38 & 140 & 18.92 & 2.529 & 1.5 & -4.4 \\ 597 & 86.66 & 7 & 152 & 18.64 & 2.528 & 0.9 & -5.6 \\ 597 & 86.66 & 39 & 180 & 18.45 & 2.509 & -0.2 & -1.9 \\ 597 & 86.66 & 8 & 194 & 18.43 & 2.536 & 0.8 & -6.1 \\ 597 & 86.66 & 24 & 2217 & 3.58 & 3.515 & 0.7 & -16.3 \\ 599 & 86.73 & 27 & 1 & 26.31 & 2.221 & 1.0 & 8.0 \\ 599 & 86.73 & 1 & 1 & ----- & 2.226 & --- & --- \\ 599 & 86.73 & 28 & 5 & 26.03 & 2.221 & 0.5 & 7.5 \\ 599 & 86.73 & 30 & 19 & 25.95 & 2.230 & 0.8 & 11.1 \\ 599 & 86.73 & 3 & 26 & 25.94 & 2.278 & 3.0 & 10.8 \\ 599 & 86.73 & 32 & 40 & 22.09 & 2.450 & 4.0 & 16.0 \\ 599 & 86.73 & 33 & 51 & 21.14 & 2.457 & 2.6 & 10.3 \\ 599 & 86.73 & 34 & 61 & 20.79 & 2.450 & 1.7 & 9.6 \\ 599 & 86.73 & 35 & 72 & 20.23 & 2.434 & 0.0 & 2.8 \\ 599 & 86.73 & 35 & 72 & 20.23 & 2.449 & 0.6 & 2.8 \\ 599 & 86.73 & 36 & 84 & 20.10 & 2.457 & 0.7 & -0.8 \\ 599 & 86.73 & 37 & 95 & 19.86 & 2.501 & 2.1 & 1.7 \\ 599 & 86.73 & 38 & 132 & 19.26 & 2.476 & 0.0 & 1.9 \\ 599 & 86.73 & 39 & 179 & 18.98 & 2.596 & 4.2 & -0.6 \\ 599 & 86.73 & 24 & 2238 & 3.54 & 3.452 & -1.3 & -14.7 \\ 601 & 86.85 & 1 & 1 & 24.28 & 2.340 & 3.1 & 8.6 \\ 601 & 86.85 & 27 & 2 & 24.21 & 2.359 & 3.8 & 6.5 \\ 601 & 86.85 & 28 & 8 & 24.15 & 2.300 & 1.2 & 8.8 \\ 601 & 86.85 & 2 & 11 & 24.07 & 2.318 & 1.8 & 6.1 \\ 601 & 86.85 & 29 & 15 & 24.09 & 2.305 & 1.3 & 7.2 \\ 601 & 86.85 & 29 & 15 & 24.09 & 2.296 & 0.9 & 7.2 \\ 601 & 86.85 & 30 & 24 & 24.09 & 2.324 & 2.1 & 7.8 \\ 601 & 86.85 & 3 & 27 & 24.14 & 2.291 & 0.8 & 7.9 \\ 601 & 86.85 & 34 & 65 & 23.01 & 2.403 & 3.7 & 13.7 \\ 601 & 86.85 & 37 & 95 & 20.52 & 2.455 & 1.4 & 5.3 \\ 601 & 86.85 & 6 & 105 & 20.34 & 2.467 & 1.6 & 2.6 \\ 601 & 86.85 & 38 & 131 & 19.37 & 2.488 & 0.7 & -4.1\end{array}$




$\begin{array}{rrrrrrrr}601 & 86.85 & 39 & 175 & 18.98 & 2.485 & -0.2 & -6.1 \\ 603 & 86.91 & 27 & 1 & 23.36 & 2.312 & 0.3 & 4.4 \\ 603 & 86.91 & 28 & 5 & 23.34 & 2.319 & 0.6 & 4.1 \\ 603 & 86.91 & 29 & 10 & 23.30 & 2.307 & 0.0 & 4.5 \\ 603 & 86.91 & 30 & 22 & 23.34 & 2.416 & 4.8 & 4.3 \\ 603 & 86.91 & 3 & 29 & 23.21 & 2.331 & 0.9 & 4.9 \\ 603 & 86.91 & 31 & 34 & 23.34 & 2.331 & 1.1 & 4.7 \\ 603 & 86.91 & 31 & 34 & 23.34 & 2.329 & 1.0 & 4.7 \\ 603 & 86.91 & 33 & 43 & 23.35 & 2.333 & 1.2 & 5.6 \\ 603 & 86.91 & 4 & 52 & 23.18 & 2.328 & 0.7 & 4.4 \\ 603 & 86.91 & 35 & 67 & 25.13 & 2.342 & 4.7 & 10.2 \\ 603 & 86.91 & 39 & 186 & 18.83 & 2.522 & 1.0 & -7.4 \\ 603 & 86.91 & 24 & 1910 & 3.99 & 3.421 & -1.0 & -16.6 \\ 604 & 87.06 & 27 & 1 & 20.56 & 2.425 & 0.2 & 4.4 \\ 604 & 87.06 & 1 & 1 & 20.58 & 2.437 & 0.8 & 4.8 \\ 604 & 87.06 & 28 & 6 & 20.53 & 2.422 & 0.1 & 3.9 \\ 604 & 87.06 & 2 & 10 & 20.47 & 2.414 & -0.4 & 4.4 \\ 604 & 87.06 & 29 & 12 & 20.47 & 2.423 & 0.0 & 4.0 \\ 604 & 87.06 & 30 & 23 & 20.54 & 2.424 & 0.1 & 3.4 \\ 604 & 87.06 & 31 & 35 & 20.54 & 2.460 & 1.7 & 5.0 \\ 604 & 87.06 & 32 & 46 & 20.45 & 2.417 & -0.3 & 3.4 \\ 604 & 87.06 & 33 & 57 & 20.49 & 2.445 & 0.9 & 3.7 \\ 604 & 87.06 & 34 & 65 & ----- & 2.433 & --- & --- \\ 604 & 87.06 & 35 & 73 & 20.47 & 2.431 & 0.3 & 3.6 \\ 604 & 87.06 & 36 & 88 & 20.48 & 2.440 & 0.7 & 4.0 \\ 604 & 87.06 & 37 & 92 & 20.41 & 2.481 & 2.2 & 2.9 \\ 604 & 87.06 & 38 & 131 & 20.36 & 2.427 & -0.1 & 2.0 \\ 604 & 87.06 & 38 & 131 & 20.36 & 2.419 & -0.4 & 2.0 \\ 604 & 87.06 & 24 & 2220 & 3.57 & 3.529 & 1.0 & -15.3 \\ 606 & 87.19 & 27 & 1 & 19.50 & 2.466 & 0.1 & 3.2 \\ 606 & 87.19 & 28 & 6 & 19.47 & 2.513 & 1.9 & 3.0 \\ 606 & 87.19 & 30 & 23 & 19.33 & 2.499 & 1.1 & 3.5 \\ 606 & 87.19 & 32 & 44 & 19.34 & 2.536 & 2.6 & 1.5 \\ 606 & 87.19 & 34 & 67 & 19.34 & 2.500 & 1.1 & 1.9 \\ 606 & 87.19 & 35 & 80 & 19.35 & 2.474 & 0.1 & 0.6 \\ 606 & 87.19 & 35 & 80 & 19.35 & 2.482 & 0.4 & 0.6 \\ 606 & 87.19 & 36 & 90 & 19.34 & 2.500 & 1.1 & 1.9 \\ 606 & 87.19 & 38 & 136 & 19.35 & 2.471 & 0.0 & 2.1 \\ 606 & 87.19 & 39 & 191 & 19.29 & 2.486 & 0.4 & 0.4 \\ 606 & 87.19 & 24 & 2229 & 3.49 & 3.461 & -1.1 & -16.3 \\ 607 & 87.24 & 27 & 2 & 19.02 & 2.492 & 0.2 & 4.6 \\ 607 & 87.24 & 29 & 11 & 19.01 & 2.486 & 0.0 & 4.9 \\ 607 & 87.24 & 29 & 11 & 19.01 & 2.492 & 0.2 & 4.9 \\ 607 & 87.24 & 31 & 34 & 18.90 & 2.508 & 0.6 & 3.0 \\ 607 & 87.24 & 32 & 44 & 18.84 & 2.487 & -0.3 & 3.8\end{array}$




$\begin{array}{rrrrrrrr}607 & 87.24 & 33 & 54 & 18.84 & 2.489 & -0.3 & 2.3 \\ 607 & 87.24 & 34 & 63 & 18.79 & 2.475 & -0.9 & 2.0 \\ 607 & 87.24 & 35 & 71 & 18.79 & 2.545 & 1.9 & 0.7 \\ 607 & 87.24 & 36 & 81 & 18.78 & 2.494 & -0.2 & 1.2 \\ 607 & 87.24 & 38 & 128 & 18.91 & 2.476 & -0.7 & 1.6 \\ 607 & 87.24 & 39 & 180 & 18.77 & 2.514 & 0.6 & 0.4 \\ 609 & 87.31 & 1 & 1 & 20.27 & 2.450 & 0.8 & 5.9 \\ 609 & 87.31 & 2 & 12 & 20.18 & 2.475 & 1.7 & 5.1 \\ 609 & 87.31 & 24 & 2178 & 3.77 & 3.455 & -0.6 & -16.5 \\ 611 & 87.41 & 1 & 1 & 22.51 & 2.463 & 5.3 & 7.5 \\ 611 & 87.41 & 28 & 5 & 22.58 & 2.412 & 3.3 & 6.6 \\ 611 & 87.41 & 29 & 11 & 22.13 & 2.421 & 2.8 & 6.8 \\ 611 & 87.41 & 30 & 20 & 22.00 & 2.446 & 3.7 & 9.4 \\ 611 & 87.41 & 3 & 23 & 22.98 & 2.399 & 3.4 & 10.0 \\ 611 & 87.41 & 31 & 32 & 21.73 & 2.447 & 3.2 & 7.2 \\ 611 & 87.41 & 32 & 41 & 21.43 & 2.480 & 4.1 & 7.9 \\ 611 & 87.41 & 34 & 60 & 20.58 & 2.472 & 2.2 & 5.2 \\ 611 & 87.41 & 34 & 60 & 20.58 & 2.458 & 1.6 & 5.2 \\ 611 & 87.41 & 35 & 70 & 20.03 & 2.485 & 1.7 & 5.1 \\ 611 & 87.41 & 36 & 81 & 19.71 & 2.459 & 0.1 & 1.4 \\ 611 & 87.41 & 37 & 91 & 19.60 & 2.483 & 0.9 & -0.7 \\ 611 & 87.41 & 38 & 126 & 19.11 & 2.477 & -0.3 & 0.5 \\ 611 & 87.41 & 39 & 175 & 18.73 & 2.549 & 1.9 & -1.6 \\ 613 & 87.49 & 1 & 1 & 25.59 & 2.297 & 3.4 & 8.4 \\ 613 & 87.49 & 27 & 1 & 25.75 & 2.279 & 2.9 & 8.9 \\ 613 & 87.49 & 2 & 11 & 24.96 & 2.277 & 1.5 & 7.3 \\ 613 & 87.49 & 29 & 11 & 25.00 & 2.282 & 1.8 & 9.5 \\ 613 & 87.49 & 30 & 23 & 24.90 & 2.286 & 1.8 & 7.8 \\ 613 & 87.49 & 3 & 27 & 24.50 & 2.304 & 1.9 & 8.1 \\ 613 & 87.49 & 31 & 33 & 23.70 & 2.340 & 2.1 & 9.2 \\ 613 & 87.49 & 32 & 43 & 23.17 & 2.353 & 1.8 & 9.0 \\ 613 & 87.49 & 33 & 52 & 21.99 & 2.410 & 2.1 & 10.0 \\ 613 & 87.49 & 35 & 72 & 20.21 & 2.507 & 2.9 & 10.2 \\ 613 & 87.49 & 36 & 84 & 19.57 & 2.500 & 1.5 & 8.9 \\ 613 & 87.49 & 37 & 92 & 19.15 & 2.486 & 0.2 & 1.7 \\ 613 & 87.49 & 38 & 116 & 18.75 & 2.547 & 1.8 & 2.1 \\ 613 & 87.49 & 39 & 163 & 18.39 & 2.510 & -0.3 & -3.4 \\ 615 & 87.55 & 1 & 2 & 27.19 & 2.280 & 5.4 & 10.2 \\ 615 & 87.55 & 28 & 8 & 27.21 & 2.205 & 2.0 & 9.8 \\ 615 & 87.55 & 2 & 13 & 27.19 & 2.205 & 1.9 & 10.0 \\ 615 & 87.55 & 30 & 26 & 25.04 & 2.323 & 3.7 & 14.1 \\ 615 & 87.55 & 33 & 48 & 21.62 & 2.438 & 2.8 & 11.1 \\ 615 & 87.55 & 35 & 71 & 20.02 & 2.514 & 2.9 & 4.2 \\ 615 & 87.55 & 36 & 82 & 19.68 & 2.486 & 1.1 & 1.2 \\ 615 & 87.55 & 37 & 93 & 19.40 & 2.489 & 0.8 & -0.1\end{array}$




$\begin{array}{rrrrrrrr}615 & 87.55 & 39 & 182 & 18.48 & 2.507 & -0.3 & -8.3 \\ 618 & 87.66 & 27 & 1 & 25.97 & 2.231 & 1.0 & 8.2 \\ 618 & 87.66 & 1 & 1 & 25.95 & 2.240 & 0.8 & 7.3 \\ 618 & 87.66 & 28 & 5 & 25.89 & 2.236 & 1.1 & 7.2 \\ 618 & 87.66 & 2 & 10 & 25.70 & 2.258 & 1.8 & 6.8 \\ 618 & 87.66 & 30 & 20 & 25.66 & 2.237 & 0.8 & 7.9 \\ 618 & 87.66 & 31 & 30 & 24.30 & 2.321 & 2.2 & 10.2 \\ 618 & 87.66 & 33 & 50 & 20.82 & 2.471 & 2.6 & 9.5 \\ 618 & 87.66 & 34 & 60 & 19.88 & 2.471 & 0.9 & 3.1 \\ 618 & 87.66 & 35 & 71 & 19.42 & 2.508 & 1.5 & -0.7 \\ 618 & 87.66 & 5 & 76 & 19.49 & 2.507 & 1.6 & 6.2 \\ 618 & 87.66 & 36 & 82 & 19.10 & 2.498 & 0.6 & 0.6 \\ 618 & 87.66 & 38 & 126 & 18.56 & 2.511 & 0.1 & -6.1 \\ 618 & 87.66 & 7 & 152 & 18.56 & 2.637 & 5.1 & -5.0 \\ 618 & 87.66 & 8 & 205 & 18.08 & 2.539 & 0.2 & -5.3 \\ 618 & 87.66 & 24 & 2229 & 3.65 & 3.460 & -0.8 & -16.3 \\ 618 & 87.66 & 24 & 2229 & 3.65 & 3.469 & -0.5 & -16.3 \\ 620 & 87.80 & 29 & 10 & 26.02 & 2.211 & 0.1 & 8.6 \\ 620 & 87.80 & 29 & 10 & 26.02 & 2.250 & 1.8 & 8.6 \\ 620 & 87.80 & 30 & 20 & 26.03 & 2.232 & 1.1 & 11.0 \\ 620 & 87.80 & 31 & 31 & 25.99 & 2.262 & 2.3 & 8.5 \\ 620 & 87.80 & 32 & 43 & 25.44 & 2.268 & 1.7 & 10.3 \\ 620 & 87.80 & 34 & 61 & 24.46 & 2.360 & 4.2 & 10.1 \\ 620 & 87.80 & 36 & 81 & 22.18 & 2.409 & 2.4 & 5.1 \\ 620 & 87.80 & 38 & 128 & 19.86 & 2.484 & 1.4 & -0.8 \\ 620 & 87.80 & 39 & 181 & 18.87 & 2.588 & 3.7 & -6.5 \\ 622 & 87.86 & 27 & 1 & 24.50 & 2.288 & 1.1 & 5.7 \\ 622 & 87.86 & 28 & 5 & 24.42 & 2.282 & 0.7 & 5.6 \\ 622 & 87.86 & 29 & 11 & 24.29 & 2.275 & 0.2 & 5.6 \\ 622 & 87.86 & 30 & 21 & 24.28 & 2.289 & 0.8 & 5.5 \\ 622 & 87.86 & 31 & 32 & 24.25 & 2.269 & -0.1 & 6.3 \\ 622 & 87.86 & 32 & 42 & 24.23 & 2.288 & 0.7 & 6.3 \\ 622 & 87.86 & 33 & 52 & 24.40 & 2.309 & 1.9 & 4.3 \\ 622 & 87.86 & 34 & 59 & 22.53 & 2.449 & 4.8 & 10.8 \\ 622 & 87.86 & 35 & 68 & 21.32 & 2.433 & 1.9 & 4.9 \\ 622 & 87.86 & 38 & 126 & 18.90 & 2.627 & 5.4 & -8.4 \\ 626 & 87.92 & 27 & 1 & 22.17 & 2.354 & 0.1 & 4.4 \\ 626 & 87.92 & 27 & 1 & 22.17 & 2.368 & 0.7 & 4.4 \\ 626 & 87.92 & 28 & 5 & 22.10 & 2.347 & -0.4 & 4.7 \\ 626 & 87.92 & 29 & 10 & 22.11 & 2.350 & -0.2 & 5.1 \\ 626 & 87.92 & 30 & 18 & 22.12 & 2.358 & 0.1 & 4.3 \\ 626 & 87.92 & 31 & 30 & 22.12 & 2.367 & 0.5 & 4.9 \\ 626 & 87.92 & 32 & 41 & 22.12 & 2.465 & 4.7 & 5.5 \\ 626 & 87.92 & 33 & 51 & 22.34 & 2.349 & 0.1 & 6.4 \\ 626 & 87.92 & 34 & 57 & 22.17 & 2.354 & 0.1 & 4.4\end{array}$




\begin{tabular}{|c|c|c|c|c|c|c|c|}
\hline 626 & 87.92 & 38 & 123 & 18.70 & 2.552 & 2.0 & -8.7 \\
\hline 628 & 88.07 & 27 & 1 & 19.87 & 2.469 & 0.8 & 4.6 \\
\hline 628 & 88.07 & 28 & 5 & 19.87 & 2.465 & 0.6 & 5.6 \\
\hline 628 & 88.07 & 29 & 10 & 19.83 & 2.477 & 1.0 & 5.7 \\
\hline 628 & 88.07 & 30 & 20 & 19.76 & 2.474 & 0.8 & 5.8 \\
\hline 628 & 88.07 & 32 & 40 & 19.72 & 2.464 & 0.3 & 4.0 \\
\hline 628 & 88.07 & 33 & 51 & 19.70 & 2.458 & 0.0 & 5.3 \\
\hline 628 & 88.07 & 34 & 61 & 19.67 & 2.472 & 0.5 & 3.9 \\
\hline 628 & 88.07 & 35 & 71 & 19.67 & 2.590 & 5.3 & 3.7 \\
\hline 628 & 88.07 & 35 & 71 & 19.67 & 2.433 & -1.0 & 3.7 \\
\hline 628 & 88.07 & 36 & 82 & 19.68 & 2.466 & 0.3 & 3.9 \\
\hline 628 & 88.07 & 37 & 92 & 19.69 & 2.584 & 5.1 & 3.1 \\
\hline 628 & 88.07 & 38 & 128 & 19.67 & 2.481 & 0.9 & 3.4 \\
\hline 631 & 88.19 & 27 & 1 & 19.14 & 2.497 & 0.6 & 2.7 \\
\hline 631 & 88.19 & 28 & 5 & 19.08 & 2.498 & 0.5 & 2.7 \\
\hline 631 & 88.19 & 28 & 5 & 19.08 & 2.497 & 0.5 & 2.7 \\
\hline 631 & 88.19 & 29 & 10 & 19.03 & 2.504 & 0.7 & 2.3 \\
\hline 631 & 88.19 & 30 & 20 & 18.99 & 2.507 & 0.7 & 2.0 \\
\hline 631 & 88.19 & 31 & 31 & 18.96 & 2.488 & -0.1 & 2.1 \\
\hline 631 & 88.19 & 32 & 41 & 18.97 & 2.483 & -0.3 & 1.7 \\
\hline 631 & 88.19 & 33 & 51 & 18.96 & 2.488 & -0.1 & 3.1 \\
\hline 631 & 88.19 & 34 & 61 & 19.00 & 2.484 & -0.2 & 1.8 \\
\hline 631 & 88.19 & 35 & 71 & 18.97 & 2.486 & -0.2 & 1.7 \\
\hline 631 & 88.19 & 36 & 81 & 18.97 & 2.496 & 0.2 & 2.5 \\
\hline 631 & 88.19 & 37 & 91 & 19.00 & 2.494 & 0.2 & 2.0 \\
\hline 631 & 88.19 & 38 & 127 & 18.98 & 2.467 & -0.9 & 1.0 \\
\hline 631 & 88.19 & 39 & 178 & 18.81 & 2.481 & -0.7 & -2.2 \\
\hline
\end{tabular}

\section{Notes:}

(1) Year-time is computed as the fraction of the year elapsed since January 1 (e.g. 85.50 is equivalent to July 1, 1985).

(2) Argon concentrations are measured in units of $10^{--4} \mathrm{Cc}$ STP $\mathrm{g}^{\wedge}-1$ seawater.

(3) Saturation anomalies are expressed in percent deviation from solubility equilibrium at the potential temperature and salinity of the sample and at a barometric pressure of 1 atmosphere. 


\section{Biographical Note}

The author was born on March 16, 1962 in Hewlett, NY. He graduated from George W. Hewlett High School in 1979. He received a B.A. in Chemistry and Physics from Harvard in 1983. Subsequently, he spent a year as a raft guide and kayak instructor. In 1984, he entered the MIT/WHOI Joint Program in Oceanography as a candidate for the degree of Doctor of Philosophy. 